Pontifícia Universidade $C_{\text {atólica }}$

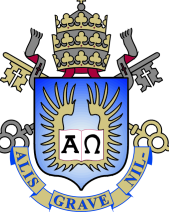

Janaina Figueira Marchesi

\title{
Healthcare staff scheduling using optimization under uncertainty and simulation
}

Tese de Doutorado

Thesis presented to the Programa de Pós-Graduação em Engenharia de Produção of PUC-Rio in partial fulfillment of the requirements for the degree of Doutor em Engenharia de Produção. Approved by the Examination Commnitee.

Advisor: Prof. Silvio Hamacher 


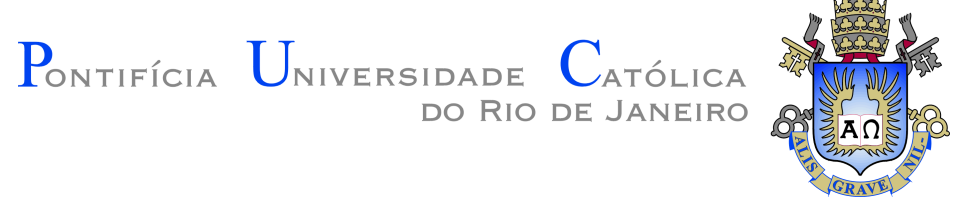

Janaina Figueira Marchesi

\section{Healthcare staff scheduling using optimization under uncertainty and simulation}

Thesis presented to the Programa de Pós-Graduação em Engenharia de Produção of PUC-Rio in partial fulfillment of the requirements for the degree of Doutor em Engenharia de Produção. Approved by the Examination Commnitee. Approved by the undersigned Examination Committee.

Prof. Silvio Hamacher Advisor

Departmento de Engenharia Industrial - PUC-Rio

Prof. Julia L. Fleck

Departamento de Engenharia Industrial - PUC-Rio

Prof. Fernando A. Bozza

Instituto de Pesquisa Clínica Evandro Chagas - Fundação

Oswaldo Cruz

Prof. Davi Michel Valladão

Departamento de Engenharia Industrial - PUC-Rio

Prof. Edilson Fernandes de Arruda

Programa de Engenharia de Produção - UFRJ

Rio de Janeiro, August the 13th, 2019 
All rights reserved.

\section{Janaina Figueira Marchesi}

JanainaMarchesi holds a Bachelor of Science degree in Industrial Engineering from Federal Institute of Education, Science and Technology of Espirito Santo, Brazil (2014) and a Master's degree in Industrial Engineering from Pontifical Catholic University of Rio de Janeiro (PUC-Rio) (2015). Since 2016, she has worked at the Institute of Technical-Scientific Software Development of PUC-Rio (Tecgraf / PUC-Rio) as researcher fellow. Janaina has experience in the area of Industrial Engineering, with emphasis on Planning, Scheduling and Control of Production and Operations, working mainly on the following topics: Planning and Scheduling of Healthcare Operations, Computer Simulation, Mathematical Programming, Optimization, Statistics, Data Analysis, Time Series, Statistical Process Control, among other.

Bibliographic data

Marchesi, Janaina Figueira

Healthcare staff scheduling using optimization under uncertainty and simulation / Janaina Figueira Marchesi; advisor: Silvio Hamacher. - 2019.

205 f: il. color. ; $30 \mathrm{~cm}$

Tese (doutorado) - Pontifícia Universidade Católica do Rio de Janeiro, Departmento de Engenharia Industrial, 2019.

Inclui bibliografia.

1. Engenharia Industrial - Teses. 2. PO em serviços de saúde;. 3. Escalonamento;. 4. Médico;. 5. Programação estocástica;. 6. Simulação de eventos discretos.. I. Hamacher, Silvio. II. Pontifícia Universidade Católica do Rio de Janeiro. Departmento de Engenharia Industrial. III. Título. 
To God, to my parents, Paulo ${ }^{\dagger}$ and Jandira, and to my brother João Paulo. 


\section{Acknowledgments}

To God, for giving me strength, to illuminate my ways and to allow me to achieve this goal.

To my family, for my education, support, affection, and dedication, and for being always by my side, helping me.

To the adviser Silvio Hamacher, for the knowledge, guides, lessons, and motivations. He is one of the most notable examples I've had in my life. I am eternally grateful for all the doors that he opened for me, his continuous support, and trust.

It was an honor for me having the opportunity to work with Prof. Kalyan S. Pasupathy and Prof. Mustafa Y. Sir at Mayo Clinic. It was an incredible experience to have the chance to work with them and his group. My sincere thanks go to them for the support and dedication to me during my stay.

To Leila, Igor, Victor, and Leonardo, for their partnership in studies and moments of relaxation that has become a friendship that I want to take forever.

To Stephanie, for patience and unconditional support, believing and making me believe in my potential during my $\mathrm{PhD}$.

To Luana, Iuri, Pedro, João Gabriel, Luiza, Gabriela, Danuza, and other colleagues, for their receptivity and collaboration in the work environment.

To the many colleagues and professors of PUC-Rio, for providing me with subsidies that allowed me to obtain a valuable academic improvement.

To CNPq, FAPERJ, Tecgraf, and PUC-Rio, for the aid granted and the excellent study environment, without which this work would not have been possible.

I want to thank to my special friends (Giovanna, Romerito, Jânia, Juliane, Bruna, my EJC's friends, my friends from PSM, and many others) and all those people that were around me providing emotional support and comfort.

To all those who, in some way, contributed to this work.

This study was financed in part by the Coordenação de Aperfeiçoamento de Pessoal de Nível Superior - Brasil (CAPES) - Finace Code 001. 


\section{Abstract}

Marchesi, Janaina Figueira; Hamacher, Silvio (Advisor). Healthcare staff scheduling using optimization under uncertainty and simulation. Rio de Janeiro, 2019. 205p. Tese de Doutorado - Departmento de Engenharia Industrial, Pontifícia Universidade Católica do Rio de Janeiro.

In this thesis, we approach the problem of healthcare staff scheduling to propose a more efficient use of existing capacity to provide timely access in different health services. We present a set of problems related to healthcare staff scheduling. The first problem seeks to reduce the door-to-doctor time in an Emergency Department; the second problem aims to reduce the waiting time of the overall treatment also in an Emergency Department; the third problem aims to provide timely access to both clinic and surgery in a specialized surgical unit. We formulate and solve two-stage stochastic programming models that seek to accurately represent the particular features that are inherent of each problem. An important aspect in healthcare problems is a large number of uncertainties involved in the processes. The incorporation of the uncertainty increases the complexity of the problem, and it, therefore, becomes computationally infeasible to consider all of the possible scenarios. We circumvent this difficulty by relying on Sample Average Approximation (SAA) to address the demand uncertainty. We also use a discrete-event simulation (DES) model to represent the problems. Finally, we apply the framework to real case studies showing that the proposed models are adaptable to different healthcare providers. Throughout the thesis, we efficiently solve the models using real cases of Brazil and USA hospitals.

\section{Keywords}

OR in health services; Staff scheduling; Physician; Stochastic programming; Discrete event simulation. 


\section{Resumo}

Marchesi, Janaina Figueira; Hamacher, Silvio. Programação de profissionais de saúde usando otimização sob incerteza e simulação . Rio de Janeiro, 2019. 205p. Tese de Doutorado Departamento de Engenharia Industrial, Pontifícia Universidade Católica do Rio de Janeiro.

Nesta tese, abordamos o escalonamento de profissionais de saúde para propor um uso mais eficiente da capacidade existente e fornecer acesso oportuno em diferentes serviços de saúde. Apresentamos um conjunto de problemas relacionados à programação de equipes de saúde. O primeiro problema procura reduzir o tempo de porta-médico em uma unidade de pronto atendimento; o segundo problema visa reduzir o tempo de espera total de tratamento também em uma unidade de pronto atendimento; o terceiro problema visa fornecer acesso oportuno à consulta clínica e à cirurgia em uma unidade cirúrgica especializada. Foram propostos e resolvidos modelos de programação estocástica de dois estágios que procuram representar com precisão as características particulares inerentes a cada problema. Um aspecto importante em problemas de saúde é o grande número de incertezas envolvidas nos processos. A incorporação da incerteza aumenta a complexidade do problema e, portanto, torna-se impossível computacionalmente considerar todos os cenários possíveis. Essa dificuldade é contornada usando a Aproximação por Média Amostral (SAA) para representar a incerteza na demanda. Modelo de simulação de eventos discretos (DES) é usado para representar os problemas. Por fim, as soluções foram aplicadas a estudos de caso reais, mostrando que os modelos propostos são adaptáveis a diferentes prestadores de serviços de saúde. Ao longo da tese, resolvemos com eficiência os modelos utilizando casos reais de hospitais no Brasil e nos EUA.

\section{Palavras-chave}

PO em serviços de saúde; Escalonamento; Médico; Programação estocástica; Simulação de eventos discretos. 


\section{Table of contents}

1 Introduction $\quad 18$

1.1 Objectives 19

$\begin{array}{lll}1.2 & \text { Thesis Organization } & 19\end{array}$

2 Background of Physician Scheduling Problem 22

2.1 Staff Scheduling Problem and Classification 22

2.1.1 Problem Definition 23

2.1.2 Modeling Framework 24

2.1.3 Implementation Issues 24

2.2 Physician Scheduling Problem 25

2.2.1 Problem Definition 26

2.2.2 Modeling Framework 28

2.2.3 Implementation Issues 31

$\begin{array}{lll}2.3 & \text { Discussion } & 36\end{array}$

$\begin{array}{lll}2.4 & \text { Conclusions } & 38\end{array}$

3 Methods $\quad 40$

3.1 Dealing with Demand Uncertainty Using Sample Average Approximation 40

3.1.1 Sample Average Approximaton 41

3.1.1.1 Lower bound estimates $\quad 42$

3.1.1.2 Upper bound estimates 43

3.1.1.3 Estimating the Gap 44

3.1.2 Scenario Generation Methods 45

3.1.2.1 Monte Carlo Sampling 45

3.1.2.2 Latin Hypercube Sampling 45

3.1.3 Evaluation of Scenario Generation's Methods 46

$\begin{array}{lll}\text { 3.1.3.1 In-sample stability } & 47\end{array}$

$\begin{array}{ll}3.1 .4 & \text { Out-of-sample stability }\end{array}$

$\begin{array}{ll}3.2 & \text { Solution framework }\end{array}$

3.2.1 Statistical analysis 48

3.2.2 Methodology using Sample average approximation to deal with uncertainty in the thesis 49

3.2.3 Two-stage stochastic programming formulation 50

3.2.4 Discrete-event simulation model 50

$\begin{array}{lll}3.3 \text { Conclusions } & 50\end{array}$

4 Physician Staffing and Scheduling for Reducing Door-to-Doctor Time in an Emergency Department $\quad 52$

4.1 Problem Description $\quad 52$

4.2 Mathematical Model Framework 58

4.3 Simulation Modeling Framework $\quad 63$

4.4 Numerical Experiments 63

4.5 Case study in the Hospital A 64

$\begin{array}{lll}\text { 4.5.1 Physician staff } & 66\end{array}$ 
$\begin{array}{lll}\text { 4.5.2 } & \text { Physician scheduling } & 67\end{array}$

4.5.3 Simulation model 67

4.5.4 Numerical results of the case study in the Hospital A 68

4.6 Case study in the Hospital B 72

\begin{tabular}{lll} 
4.6.1 Physician staff & 74 \\
\hline
\end{tabular}

$\begin{array}{lll}\text { 4.6.2 Physician scheduling } & 75\end{array}$

4.6.3 Simulation model 76

4.6.4 Numerical results of the case study in the Hospital B 76

4.7 Discussion 80

4.8 Conclusions $\quad 82$

5 Resources Staffing and Scheduling to Reduce Waiting Time in an Emergency Department $\quad 83$

5.1 General Mathematical Model $\quad 88$

$\begin{array}{ll}\text { 5.2 Simulation Modeling Framework } & 97\end{array}$

5.3 Numerical Experiments 98

5.4 Case study 98

$\begin{array}{ll}\text { 5.4.1 Simulation model } & 101\end{array}$

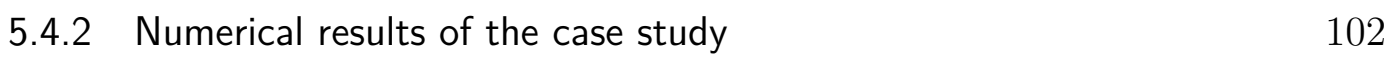

$\begin{array}{lll}5.5 \text { Conclusions } & 106\end{array}$

6 Coordinating physician scheduling for clinic and surgery appointments to deliver timely access in a specialized surgical unit $\quad 107$

6.1 Problem Description 107

6.2 Mathematical Model Framework 113

$\begin{array}{ll}6.3 \text { Numerical Experiments } & 115\end{array}$

$\begin{array}{lll}6.3 .1 & \text { Case Study } & 116\end{array}$

$\begin{array}{lll}6.3 .2 & \text { Results } & 118\end{array}$

$\begin{array}{lll}6.3 .3 & \text { Discussion } & 121\end{array}$

$\begin{array}{ll}\text { 6.3.4 Conclusions } & 123\end{array}$

7 Conclusions $\quad 124$

$\begin{array}{ll}7.1 \text { Future Perspectives } & 126\end{array}$

$\begin{array}{ll}\text { Bibliography } & 128\end{array}$

A Complete account of the studies screened, assessed for eligibility, and included in the literature review 136

B Detailed account of the constraints defined in the reviewed Physician Scheduling Problem formulations

C Assessment of planning horizon, shift types, personnel characteristics, performance measures, and constraints found in the surveyed Physician Scheduling Problem papers

D Extra material from the Hospital A's case study from Chapter $4 \quad 156$

E Size of the model from the Hospital A's case study from Chapter $4 \quad 158$ 
F Extra SAA material from the Hospital A's case study

G Numerical results from the Hospital A's case study using historical data 168

H Size of the model from the Hospital B's case study from Chapter $4 \quad 173$

I Extra SAA material from the Hospital B's case study 174

J Numerical results of the Hospital B's case study using historical data 194

K Extra material from the Hospital B's case study from Chapter $4 \quad 197$

L Extra material from the case study of Chapter $5 \quad 200$

M Size of the model from the Hospital B's case study from Chapter 5202

N Size of the model from Chapter $6 \quad 203$

O Extra material from the Chapter $6 \quad 204$ 


\section{List of figures}

$\begin{array}{lll}\text { Figure 3.1 Solution framework } & 48\end{array}$

Figure 4.1 Simplified queue model that represents the process in the $\mathrm{ED}$

Figure 4.2 Example of PLAN_HORIZON and PLAN_PERIOD behavior

Figure 4.3 Shift distribution adopted by Clinical Medicine in the ED of Hospital A (24 hour clock format)

Figure 4.4 Average waiting time per hour in Hospital A

Figure 4.5 Average waiting time in Hospital A comparing the current empirically-defined schedule of ED in the Hospital A and the schedule from the proposed model

Figure 4.6 Histogram of frequency distribution of idle capacity (negative values) or queue (positive values) for the current schedule and the schedule generated by model using SAA

Figure 4.7 Shifts distribution adopted by Clinical Medicine in the ED of Hospital B (24 hour clock format)

Figure 4.8 Average waiting time per hour in Hospital B

Figure 4.9 Average waiting time in Hospital B comparing the current empirically-defined schedule of ED in the Hospital B and the schedule from the proposed model

Figure 4.10 Histogram of frequency distribution of idle capacity (negative values) or queue (positive values) for the current schedule and the schedule generated by model using SAA

Figure 5.1 Complete flow that represents the process in the ED

Figure 5.2 General queue model that represents the process in the $\mathrm{ED}$

Figure 5.3 Transfer rate of patients from one treatment stage to another in the ED

Figure 6.1 General patient flow in a surgical specialty division

Figure 6.2 Example of physician scheduling in a surgical specialty division

Figure 6.3 Example of the planning horizon considered in the case study

Figure $6.4 \beta$ factor vs. average number of patients waiting: (a) for clinic appotinments; (b) for surgery; (c) total of patients waiting; (d) total number of patients waiting without extreme values of $\beta 0$ and 1

Figure 6.5 Average number of patients waiting for clinic appotinments vs. Average number of patients waiting for surgery

Figure 6.6 Comparation between the average number of patients waiting using the proposed model with $\beta$ factor $=0.6$ and the observed schedule (a) for clinic appointments; (b) for surgery 
Figure A.1 Stepwise data collection process for physician scheduling 138

Figure D.1 Fit test of the patients arrival distribution in the Hospital A156

Figure F.1 Color scale used in the dominant solution analysis 161

Figure G.1 Histogram of frequency distribution of idle capacity (negative values) or queue (positive values) for (a) current schedule; (b) schedule generated by Model 1

Figure J.1 Histogram of frequency distribution of idle capacity (negative values) or queue (positive values) for (a) current schedule; (b) schedule generated by Model 1

Figure K.1 Fit test of the patients arrival distribution in the Hospital A198 


\section{List of tables}

Table 2.1 Complete list of surveyed PSP papers 25

$\begin{array}{lll}\text { Table } 2.2 & \text { General model variables } & 27\end{array}$

Table 2.3 Quantity of PSP papers that account for different planning horizons 28

Table 2.4 Quantity of PSP papers that account for different performance measures

Table 2.5 Quantity of PSP papers that account for different constraint categories

Table 2.6 Implementation issues accounted for in PSP papers 32

Table 4.1 Summary of contributions from the Chapter $4 \quad 57$

Table 4.2 Sets, subsets and corresponding domains 58

Table 4.3 Model parameters $\quad 59$

Table 4.4 Model variables $\quad 60$

Table 4.5 Experiment results: in-sample and out-of-sample analysis and estimative of the optimality gap

Table 4.6 Discrete-event simulation results for case study in the Hospital A using SAA $\quad 70$

Table 4.7 Experiment results: in-sample and out-of-sample analysis and estimative of the optimality gap

Table 4.8 Discrete-event simulation results for case study in the $\begin{array}{ll}\text { Hospital B using SAA } & 78\end{array}$

Table 5.1 Summary of contributions from the Chapter $5 \quad 87$

Table 5.2 Sets, subsets and corresponding domains of the general model

Table 5.3 General model parameters $\quad 92$

Table 5.4 General model variables $\quad 92$

Table 5.5 Experiment results: estimative of the optimality gap and confidence interval 102

Table 5.6 Values of the RP, WS and EEV 103

Table 5.7 Values of indicators used to estimate the benefits of considering uncertainty in the model 104

$\begin{array}{lll}\text { Table } 5.8 & \text { Discrete-event simulation results } & 105\end{array}$

Table 6.1 Summary of contributions from the Chapter 6 112

Table 6.2 Sets, subsets and corresponding domains 113

Table 6.3 Model parameters 113

Table 6.4 Model variables 114

$\begin{array}{lll}\text { Table } 6.5 & \text { Initial queue for surgery per physician } & 117\end{array}$

Table 6.6 Gap, lower and upper bound of SAA experiments 118

Table 6.7 Confidence interval of the optimal gap 119

Table B.1 Time related constraints for PSP 140

Table B.2 Weekend related constraints for PSP 142 
Table B.3 Shift/pattern sequencing constraints for PSP 142

Table B.4 Coverage related constraints for PSP 144

Table B.5 Preference/Fairness related constraints for PSP 146

Table B.6 Other constraints for PSP 147

Table C.1 Planning horizon, shift types, and personnel characteristics found in surveyed PSP papers 150

Table C.2 Performance measures found in surveyed PSP papers 151

Table C.3 Constraints found in surveyed PSP papers 153

Table D.1 Current schedule of physicians in the Hospital A 156

Table D.2 Average interval between arrival per hour and day of week in ED of Hospital A 157

Table E.1 Size of the model from the case study in the Hospital A 158

Table F.1 Confidence interval of the optimal gap from case study in the Hospital A 159

Table F.2 Number of solutions using Monte Carlo Sampling method 160

Table F.3 Number of solutions using Latin Hypercube Sampling method

Table F.4 Analysis of dominant solution from Monte Carlo Sampling method using 100 scenarios

Table F.5 Analysis of dominant solution from Monte Carlo Sampling method using 200 scenarios

Table F.6 Analysis of dominant solution from Monte Carlo Sampling method using 300 scenarios

Table F.7 Analysis of dominant solution from Monte Carlo Sampling method using 400 scenarios

Table F.8 Analysis of dominant solution from Monte Carlo Sampling method using 500 scenarios

Table F.9 Analysis of dominant solution from Monte Carlo Sampling method using 600 scenarios

Table F.10 Analysis of dominant solution from Monte Carlo Sampling method using 700 scenarios

Table F.11 Analysis of dominant solution from Monte Carlo Sampling method using 800 scenarios

Table F.12 Analysis of dominant solution from Monte Carlo Sampling method using 900 scenarios

Table F.13 Analysis of dominant solution from Monte Carlo Sampling method using 1000 scenarios

Table F.14 Analysis of dominant solution from Latin Hypercube Sampling method using 100 scenarios

Table F.15 Analysis of dominant solution from Latin Hypercube Sampling method using 200 scenarios

Table F.16 Analysis of dominant solution from Latin Hypercube Sampling method using 300 scenarios

Table F.17 Analysis of dominant solution from Latin Hypercube Sampling method using 400 scenarios 
Table F.18 Analysis of dominant solution from Latin Hypercube Sampling method using 500 scenarios

Table F.19 Analysis of dominant solution from Latin Hypercube Sampling method using 600 scenarios

Table F.20 Analysis of dominant solution from Latin Hypercube Sampling method using 700 scenarios

Table F.21 Analysis of dominant solution from Latin Hypercube Sampling method using 800 scenarios

Table F.22 Analysis of dominant solution from Latin Hypercube Sampling method using 900 scenarios

Table F.23 Analysis of dominant solution from Latin Hypercube Sampling method using 1000 scenarios

Table G.1 Computational results to case study in the Hospital A 168

Table G.2 Discrete-event simulation results for Model 1

Table G.3 Discrete-event simulation results for Model 2

Table H.1 Size of the model from the case study in the Hospital B 173

Table I.1 Confidence interval of the optimal gap from case study in the Hospital B

Table I.2 Number of solutions using Monte Carlo Sampling method 176

Table I.3 Number of solutions using Latin Hypercube Sampling method

Table I.4 Analysis of dominant solution from Monte Carlo Sampling method using 100 scenarios

Table I.5 Analysis of dominant solution from Monte Carlo Sampling method using 200 scenarios

Table I.6 Analysis of dominant solution from Monte Carlo Sampling method using 300 scenarios

Table I.7 Analysis of dominant solution from Monte Carlo Sampling method using 400 scenarios

Table I.8 Analysis of dominant solution from Monte Carlo Sampling method using 500 scenarios

Table I.9 Analysis of dominant solution from Monte Carlo Sampling method using 600 scenarios

Table I.10 Analysis of dominant solution from Monte Carlo Sampling method using 700 scenarios

Table I.11 Analysis of dominant solution from Monte Carlo Sampling method using 800 scenarios

Table I.12 Analysis of dominant solution from Monte Carlo Sampling method using 900 scenarios

Table I.13 Analysis of dominant solution from Monte Carlo Sampling method using 1000 scenarios

Table I.14 Analysis of dominant solution from Latin Hypercube Sampling method using 100 scenarios

Table I.15 Analysis of dominant solution from Latin Hypercube Sampling method using 200 scenarios 
Table I.16 Analysis of dominant solution from Latin Hypercube Sampling method using 300 scenarios

Table I.17 Analysis of dominant solution from Latin Hypercube Sampling method using 400 scenarios

Table I.18 Analysis of dominant solution from Latin Hypercube Sampling method using 500 scenarios

Table I.19 Analysis of dominant solution from Latin Hypercube Sampling method using 600 scenarios

Table I.20 Analysis of dominant solution from Latin Hypercube Sampling method using 700 scenarios

Table I.21 Analysis of dominant solution from Latin Hypercube Sampling method using 800 scenarios

Table I.22 Analysis of dominant solution from Latin Hypercube Sampling method using 900 scenarios

Table I.23 Analysis of dominant solution from Latin Hypercube Sampling method using 1000 scenarios

Table J.1 Computational results to case study in the Hospital B

Table J.2 Discrete-event simulation results for case study in the Hospital B

Table K.1 Current schedule of physicians in the Hospital B

Table K.2 Average interval between arrival per hour and day of week for first general assessment in ED of Hospital B

Table L.1 Average interval between arrival per hour and day of week in ED of hospital studied

Table M.1 Size of SAA's experiments from Chapter 5

Table N.1 Size of SAA's experiments

Table O.1 Results summary about average queue, capacity and patients seen in clinic and surgery service 
"If you would attain to what you are not yet, you must always be displeased by what you are. For where you are pleased with yourself there you have remained. Keep adding, keep walking, keep advancing."

St. Augustine, S. 169, 18. 


\section{Introduction}

Increased attention about preventive care and the aging population have raised demand for health services over the last few decades [Granja et al., 2014]. Healthcare providers face a significant challenge in providing timely access to care. Timely access is important not only because it is an essential determinant of patient satisfaction but also because it affects the medical outcomes [Gupta and Denton, 2008]. Patients often experience serious delays due to capacity constraints, and highly variable patient demands [Green, 2005]. Adding capacity is a drastic strategic measure and often infeasible due to cost pressures, regulatory constraints, or shortage of appropriate personnel [Green, 2005; Hans et al., 2012]. Therefore, to meet demand properly, healthcare administrators often struggle to use existing capacity more efficiently, since tactically allocating and organizing the available resources may be more effective and cheaper [Hans et al., 2012; Turhan and Bilgen, 2017]. In this context, mathematical techniques, including Operational Research, offer particularly suitable tools for tackling modern healthcare challenges such as process design, capacity allocation, admission control, appointment, and staff scheduling [Barz and Rajaram, 2015; Granja et al., 2014; Turhan and Bilgen, 2017].

Staff scheduling (also termed personnel scheduling or rostering) is the process of creating work timetables for the staff to ensure that an organization will satisfy a certain demand for goods or services. Healthcare staff scheduling is of particular importance because inadequate scheduling leads to inappropriate patient treatment, decreasing both patient and provider satisfaction [Ganguly et al., 2014]. Satisfying personnel demands in the healthcare sector is, however, a particularly complex task due to the multi-skill nature of human resources in this sector.

An important feature in healthcare problems is a large number of uncertainties involved in the processes, such as: non-deterministic patient arrival times and service durations, emergency care, among others. Since the 1960s, healthcare staff scheduling has been addressed by using management science and operations research, surveys on the topic are given in [Burke et al., 2004; Cheang et al., 2003; Erhard et al., 2018]. Although the research literature in this fields is very extensive, few studies have included uncertainty in the 
formulation. However, if the tasks are subject to uncertainty, it is important to account for them when perform the optimization of the schedule for a given period.

\section{1}

\section{Objectives}

In this thesis, our principal objective is to address the problem of healthcare staff scheduling to propose a more efficient use of existing capacity to provide timely access in different health services, taking into consideration the effects of demand uncertainty. In order to accomplish this objective, we present a set of problems related to healthcare staff scheduling, its particular features, and a complete framework to solve each one of the problems. Such a framework consists of a mathematical model to represent the problem, techniques to include the uncertainty in demand into this model, and techniques to evaluate the results of the models proposed.

As for secondary objectives, we list the following: we consider the use of Sample Average Approximation (SAA) as a technique to address the demand uncertainty, and we show how one can use this technique to deal with the uncertainty; we present the Monte Carlo and Latin Hypercube as sampling methods to use with the SAA technique, and we evaluate the performance of both to handle the scenario generation. In addition to that, we use a discreteevent simulation model to represent the problems and evaluate the results given by the model. Another objective is the successful application of the framework to real case studies showing how the proposed models are adaptable to different health care providers. Throughout the thesis, we show how one can consider the uncertainty in the problems presented and efficiently solve them using real cases of Brazil and USA hospitals.

\section{2}

\section{Thesis Organization}

This thesis is organized into seven chapters, as follows:

In Chapter 2, we present a background of health care staff scheduling. We summarize the findings of existing studies, propose a taxonomy of existing solution methods, identify limitations, and point directions for future research.

In Chapter 3, we discuss the Sample Average Approximation (SAA) to deal with the uncertainty. We show two sampling methods to handle the scenario generation and how to evaluate the scenario generation methods. Then, we show the methodology steps adopted in this thesis to deal with 
uncertainty. We finish that chapter presenting the solution framework proposed in this thesis to include the methodology steps to deal with uncertainty.

In Chapter 4, we present the 'Physician Staffing and Scheduling for Reducing Door-to-Doctor Time in an Emergency Department' problem, giving details about the context considered and describing the assumptions that are made. Our contributions is related to the fact that we not only correctly determine the number of physicians in service during each shift, for each day of the planning horizon, but we also assign individual physicians to each shift following the service requirements and the contractual agreements, taking into consideration uncertainty in patient arrival patterns. In Chapter 4, we address these problems in an integrated manner, i.e., by performing both staffing and scheduling together. Moreover, we present a model that may perform staffing and scheduling, either in a cyclic or non-cyclic manner; we model multiple overlapped shifts, and different physician skill categories; and we treat physicians individually considering preferences and restrictions. We present a model formulation developed for the problem based on twostage stochastic programming. We apply the model in two real case studies in Brazilian hospitals. We solve the problem using scenarios generating by SAA technique, which results suggest that using the proposed technique, we can efficiently obtain solutions that are statistically guaranteed to be close enough to the true optimal solution.

In Chapter 5, we present the 'Resources Staffing and Scheduling to Reduce Waiting Time in an Emergency Department' problem and its characteristics. In this Chapter, we extend the problem of Chapter 4 to consider several stages of treatment in an ED, consider physicians and nurse as human resourses, and consider beds and chairs as physical resources while maintain all contributions presented before. We present the model formulation developed for the problem using two-stage stochastic programming and SAA to deal with the uncertainty. We close this chapter providing numerical results based on the real case of a Brazilian hospital.

In Chapter 6, we present the 'Coordinating physician scheduling for clinic and surgery appointments to deliver timely access in a specialized surgical unit' problem and its specific features. Here the contibutions is ralated by the fact that the concept of coordinating physician scheduling to clinic and surgery appointments addressing the uncertainty in demand is unique and novel. We proposed three prioritization policies that varies according to how the surgical unit want/need to handle the access to services. Moreover, the model proposed also considers the continuous of care between patient-surgeon. We also develop a mathematical model based on two-stage stochastic programming and use 
SAA to handle the uncertainty in demand. We end this chapter presenting numerical results of a real case study on a hospital in the USA.

In Chapter 7, we make the final considerations, we assert the contributions made, and we point future directions for further development of this work. 


\section{2}

\section{Background of Physician Scheduling Problem}

Health staff (nurses and physicians) share many general features but differ significantly with regards to employment contracts and regulations. As a result, the problem of health staff scheduling has been traditionally addressed in two separate instances: Nurse Scheduling Problem (NSP) and Physician Scheduling Problem (PSP). The former has been addressed since the 1960s and is currently the most investigated staff scheduling problem in healthcare; recent comprehensive surveys on the topic are given in [Burke et al., 2004; Cheang et al., 2003]. In contrast, significantly fewer studies have addressed PSP, with early works dating from the 2000s (see e.g. Erhard et al. [2018]). As in this thesis we are mainly interested in the PSP, in this Chapter we aim to present a background related to this problem.

To select related literature, we used the Scopus database ([Scopus, 2017]), which is the largest online database of peer-reviewed literature and includes MEDLINE. Our search for PSP papers was done for studies published up to January of 2017, yielding a total of 40 papers. A complete account of the studies screened, assessed for eligibility, and included in this review, with reasons for exclusions at each stage, is given in Appendix A.

Hence, in this chapter, we provide a systematic review of the problems of nurse and physician scheduling in which techniques, solution methods, and characteristics of the problem are analyzed, and trends and potential future research directions are identified. First, we integrate and summarize the findings of existing studies using a systematic approach. Secondly, we propose a taxonomy of existing solution methods based on three descriptive dimensions. Thirdly, we identify limitations associated with existing solution techniques and discuss trends and suggestions for future research directions.

\section{1}

\section{Staff Scheduling Problem and Classification}

To avoid ambiguity with regards to terminology, we begin with some important definitions. Shift is a set of consecutive work periods within a day that usually have a start time and well-defined order. Roster is a combination of shifts and days off assignments that encompasses a fixed period of time, 
frequently called a line-of-work [Brunner, 2010]. Staffing is the step that immediately precedes staff scheduling and involves determining the number of personnel with a given set of skills that are needed to meet certain service demands [Burke et al., 2004]. Finally, scheduling is performed by assigning individual staff members to shifts to meet required staffing levels at different times; duties are then assigned to individuals during their shifts [Ernst et al., 2004]. At this point, it is important to note that scheduling is the allocation of resources to objects in space-time, while rostering is the placing of resources into slots in a pattern [Gondane and Zanwar, 2012]. Of note, although rostering has been classified as a special case of scheduling, as in da Silva Rocha [2013], these terms have been inconsistently used in literature. For this review, we consider them to be synonymous, and will henceforth use them interchangeably whenever no confusion arises.

The Dantzig set covering formulation and its variations [Ernst et al., 2004] provided one of the earliest modeling frameworks for the staff scheduling problem. The basic concepts of scheduling problems, as well as some facets of staff scheduling, are discussed in [Blöchliger, 2004], while general surveys of staff scheduling can be found in [ Bergh et al., 2013; Alfares, 2004; Baker, 1976; Bechtold et al., 1991; Ernst et al., 2004].

One of the most studied applications of personnel scheduling involves healthcare staff, as surveyed in [Fries, 1976]. However, a variety of formulations exist for the healthcare staff scheduling problem because different healthcare institutions around the world have diverse administrative procedures [Burke et al., 2004]. In light of this, we propose a taxonomy of healthcare staff scheduling that is general enough to classify much of the work in this area. Our classification extends those of [Burke et al., 2004] and [Van Huele and Vanhoucke, 2014] and considers three descriptive dimensions of the healthcare staff scheduling problem: problem definition, modeling framework, and implementation issues.

\subsection{1}

\section{Problem Definition}

The features that define the healthcare staff scheduling problem are planning horizon, shift types, and personnel characteristics. The planning horizon refers to the time interval over which scheduling is done. Shift types are defined in terms of periodicity (cyclic or acyclic shifts), overlay (overlapping or non-overlapping shifts), and restriction of start/end times (fixed or definable shifts). In cyclic shifts, all employees within a certain category do exactly the same line of work (pattern or tour), which is repeated every planning period. 
In the case of acyclic shifts, the lines of work are completely independent, and a new schedule is generated for each planning period. Overlapping shifts are adjacent shifts that share part of the work period, while non-overlapping shifts are adjacent shifts with no intersection of hours. Fixed shifts are those with a well-defined beginning, length, and end, in contrast to definable shifts, where the length, start, and end times are decision variables of the model. Finally, personnel characteristics refer to information on how many skill categories are considered and which scheduling rules must be observed for the different categories (i.e., whether categories will be scheduled separately or in substitution). When substitution is allowed, staff with higher skill categories may be allowed to undertake jobs that are normally carried out by lower skilled employees; alternatively, only some staff may be allowed to replace staff from another skilled category in a user-defined way.

\subsection{2}

\section{Modeling Framework}

The modeling framework is defined in terms of performance measures and constraints. With regards to the performance measure, existing works are compared based on their choice of objectives. The type and amount of constraints defined in each formulation are analyzed by distinguishing between hard and soft constraints. Inability to satisfy the former will imply in infeasibility, while the latter are allowed to be violated but a penalty must be assured if there is a violation.

\subsection{3}

\section{Implementation Issues}

The issues contemplated in our analysis include a selection of solution techniques, incorporation of uncertainty, and research applicability. Existing works are compared in terms of their choice of solution technique, with options ranging from exact methods (i.e., mathematical programming) to heuristics and metaheuristics. We also compare works based on whether/how they were able to incorporate some kind of uncertainty into their formulation. Finally, research applicability refers to whether or not existing formulations were applied to real healthcare systems, and whether or not real data was used to generate measurable results. 


\section{2}

\section{Physician Scheduling Problem}

A total of 40 published journal articles dealing with Physician Scheduling Problem (PSP) were analyzed in this review, as shown in Table 2.1. The header "Country" in Table 2.1 refers to the name of the country where case studies were run, and the term "n.a." is used to designate works in which no such case studies were run, and only theoretical data was used to test the models. As can be seen in Table 2.1, 30\% of papers conducted studies in the USA, followed Germany (12,5\%), Turkey (12,5\%), and Canada (10\%). Of note, the vast majority of existing PSP works focus on resident scheduling, seldom addressing important distinguishing features such as physician preferences. In what follows, we scrutinize this issue and compare the selected PSP papers in terms of the descriptive dimensions presented in Section 2.1.

Table 2.1: Complete list of surveyed PSP papers

\begin{tabular}{cc}
\hline Paper & Country \\
\hline Bard et al. [2013] & USA \\
Bard et al. [2014] & USA \\
Bard et al. [2016] & USA \\
Baum et al. [2014] & USA \\
Beaulieu et al. [2000] & Canada \\
Bowers et al. [2016] & USA \\
Bruni and Detti [2014] & Italia \\
Brunner and Edenharter [2011] & Germany \\
Brunner et al. [2009] & Germany \\
Brunner et al. [2011] & Germany \\
Carrasco [2010] & Spain \\
Carter and Lapierre [2001] & Canada \\
Cohn et al. [2009] & USA \\
Day et al. [2006] et al. & USA \\
Elomri et al. [2015] & Qatar \\
Ferrand et al. [2011] & USA \\
Fügener et al. [2015] & Germany \\
Gendron et al. [2005] & n.a \\
Güler [2013] & Turkey \\
Güler et al. [2013] & Turkey
\end{tabular}




\begin{tabular}{cc} 
Gunawan and Lau [2010] & Singapore \\
Gunawan and Lau [2013] & Singapore \\
Hidri and Labidi [2016] & Saudi Arabia \\
Huang et al. [2016] & Taiwan \\
Kazemian et al. [2014] & USA \\
Lo and Lin [2011] & Taiwan \\
Rosocha et al. [2015]. & Slovakia \\
Savage et al. [2015] & Canada \\
Shamia et al. [2015] & Qatar \\
Sherali et al. [2002] & n.a \\
Smalley and Keskinocak [2016] & USA \\
Smalley et al. [2015] & USA \\
Stolletz and Brunner [2012] & Germany \\
Topaloglu [2006] & Turkey \\
Topaloglu [2009] & Turkey \\
Topaloglu and Ozkarahan [2011] & Turkey \\
Turner et al. [2013] & n.s \\
Van Huele and Vanhoucke [2014] & Belgium \\
Wang et al. [2007] & n.a \\
White and White [2003] & Canada \\
\hline
\end{tabular}

n.s. - not specified; n.a. - not applicable

\subsection{1}

\section{Problem Definition}

A summary of information on shift types and personnel characteristics of studies dealing with PSP is given in Table 2.2. The column "Total" displays the number of papers (out of the 40 reviewed PSP papers) that account for any given feature, and table headers include a single letter under parenthesis, which we will subsequently use in reference to the headers. As seen in row (A) of Table 2.2, PSP formulations commonly deal with one or two shifts. Furthermore, PSP studies that admit a user-defined number of shifts also allow for the shift start time and shift length to be defined by the user, the only exception being the model in [Kazemian et al., 2014], in which the start time is fixed. From rows (B) and (C), we note that the majority of PSP models consider fixed shift start time and length, while a small number of them [Brunner et al., 2009, 2011; Brunner and Edenharter, 2011; Stolletz and Brunner, 2012] perform flexible shift scheduling, i.e., shifts are constructed implicitly on a period-by-period basis over the day and shift-building rules 
are implemented as constraints. Additionally, as seen in row (D), most PSP studies do not account for overlapping shifts, and a number of those that do so address the case of on-call or medical consultation shifts (e.g., the models in [Bowers et al., 2016; Topaloglu and Ozkarahan, 2011] consider three shifts, two of them traditional and one on-call 24 hour shift; the authors in [Beaulieu et al., 2000; Gunawan and Lau, 2013] model three traditional shifts and one medical consultation shift of $4 \mathrm{~h}$ ). Finally, observe the row (E) in Table 2.2, we note that approximately the same ratio of studies adopt cyclical shifts in PSP (7.5\%) and NSP (6.5\%).

In terms of personnel characteristics, shown in row $(\mathrm{F})$ of Table 2.2, no PSP studies were found in which the user is allowed to define substitutions or the number of skill categories. Additionally, hierarchical substitution is considered only in Brunner and Edenharter [2011]; Sherali et al. [2002]; White and White [2003]. Althought PSP studies rarely address substitutability, a greater proportion of PSP models (42.5\%) account for three or more skill categories. This comes as no surprise, however, given that most PSP studies deal with resident scheduling.

Table 2.2: General model variables

\begin{tabular}{|c|c|c|}
\hline Features & Definition & Total \\
\hline \multirow{5}{*}{ Shifts number (A) } & 1 & 6 \\
\hline & 2 & 15 \\
\hline & 3 & 8 \\
\hline & 4 or more & 5 \\
\hline & user-definable & 5 \\
\hline \multirow{2}{*}{ Shift length (B) } & fixed & 35 \\
\hline & user-definable & 6 \\
\hline \multirow{2}{*}{ Shift start (C) } & fixed & 37 \\
\hline & user-definable & 4 \\
\hline \multirow{2}{*}{ Shift overlapping (D) } & non-overlapping & 27 \\
\hline & overlapping & 14 \\
\hline \multicolumn{2}{|l|}{ Cyclical shifts (E) } & 3 \\
\hline \multirow{7}{*}{ Skill categories $(\mathrm{F})$} & 1 & 18 \\
\hline & 2 & 6 \\
\hline & 3 & 8 \\
\hline & 4 or more & 9 \\
\hline & user-definable & 7 \\
\hline & schedule separately & 38 \\
\hline & hierarchical substitution allowed & 3 \\
\hline
\end{tabular}


Table 2.3 present the planning periods consider in works addressing PSP. The most common planning horizon for PSP is 1 month (used in approximately $25 \%$ of the surveyed studies), but a significant number of PSP models use longer time horizons such as 3 months [Baum et al., 2014; Güler, 2013], 51 weeks [Smalley et al., 2015], and 1 year [Carrasco, 2010; Cohn et al., 2009; Ferrand et al., 2011; Smalley and Keskinocak, 2016]. It is also interesting to note instances in which scheduling is performed over small consecutive time intervals that span a longer planning period. For example, in [Cohn et al., 2009] planning is performed weekly over a time horizon of one year, and in [Bard et al., 2013, 2014] monthly planning is performed over the same time horizon. Finally, it is worth mentioning that user-defined planning horizons are less frequently verified in PSP. Moreover, most of the PSP works that allow for user-definable planning periods deal with more than one instance of the problem and use different planning horizons for each instance.

Table 2.3: Quantity of PSP papers that account for different planning horizons

\begin{tabular}{lc}
\hline Planning Horizon & Total \\
\hline 1 day & 2 \\
5 days & 1 \\
1 week & 3 \\
2 weeks & 3 \\
4 weeks & 7 \\
1 month & 10 \\
2 months & 2 \\
9 weeks & 1 \\
3 months & 2 \\
51 weeks & 1 \\
1 year & 4 \\
user-definable & 5 \\
\hline
\end{tabular}

\subsection{2}

\section{Modeling Framework}

In terms of performance measure (Table 2.4), the trend verified among PSP is that most models seek to minimize penalties related to soft constraints. The second most common objective addresses physician preferences, generally defined in terms of two different objectives: maximizing staff preference/satisfaction and minimizing total preference cost. Another frequently occurring goal deals with minimizing overtime costs to address the issue of workload.

Min - Minimize; Max - Maximize

Some issues such as coverage and service quality are particularly relevant in staff scheduling problems. PSP models that take this into account typically 
Table 2.4: Quantity of PSP papers that account for different performance measures

\begin{tabular}{lcc}
\hline Objective & Min/Max & Total \\
\hline Soft Constraint Violations & Min & 16 \\
Preferences & Max & 8 \\
Preference Cost & Min & 6 \\
Overtime Assignment Costs & Min & 6 \\
Cost of outside physicians & Min & 5 \\
Understaffing & Min & 4 \\
Hour worked by residents & Max & 3 \\
Fairly in the scheduling & Max & 3 \\
Remuneration & Min & 3 \\
Patient handoff continuity & Min & 3 \\
Overstaffing & Min & 2 \\
Deviation from Equality & Min & 8 \\
Other & - & 8 \\
\hline
\end{tabular}

minimize understaffing and/or overstaffing. Topics of fairness and patient handoffs continuity are also translated into performance measures to ensure equity and decrease the frequency of transitions in patient care.

Finally, some studies propose more particular objectives. The model from Stolletz and Brunner [2012] aims at minimizing paid out time (i.e., not compensated overtime) in addition to minimizing overtime and the number of outside physicians. The authors in [Gunawan and Lau, 2013] focus on unscheduled duties beyond preferences, while those in [Smalley and Keskinocak, 2016] choose to minimize the variance of intervals between consecutive physician duties. To construct annual block schedules for family medicine, the authors in [Bard et al., 2016] generate monthly schedules for the house staff that minimize the maximum absolute deviation between the number of patients that can be seen during any session over the week and minimize the number changes that result from reassigning clinic sessions over the week.

For the purposes of our analysis, PSP constraints were grouped into five categories: time related constrains; weekend related constrains; shifts pattern/sequencing constrains; coverage related constraints; preference/fairness related constraints. A summary of this classification for the PSP studies included in this review is given in Table 2.5. The most common categories of constraints in PSP are time-related constraints and constraints related to shift pattern/sequencing. Among the former, the most frequently occurring constraints include a minimum interval between shift allocation, maximum consecutive working days, and maximum/minimum number of hours a physician must work in period. With regards to the latter, the majority of constraints are cast as assigning some shift pattern, assigning specific shift patterns to skills and keeping to a maximum number of certain shift types. 
Table 2.5: Quantity of PSP papers that account for different constraint categories

\begin{tabular}{rcc}
\hline Constraint category & Type & Total \\
\hline Time related & HC & 40 \\
\cline { 2 - 3 } Weekend related & SC & 16 \\
\cline { 2 - 3 } & HC & 12 \\
\hline \multirow{2}{*}{ Shifts pattern/sequencing } & SC & 6 \\
\hline \multirow{2}{*}{ Cover related } & HC & 22 \\
\cline { 2 - 3 } & SC & 10 \\
\cline { 2 - 3 } Preference/Fairness related & SC & 32 \\
\hline & HC & 10 \\
\hline \multirow{2}{*}{ Otherd } & SC & 28 \\
\cline { 2 - 3 } & HC & 9 \\
\hline
\end{tabular}

HC - Hard Constraints; SC - Soft Constraints

As in most staff scheduling problems, PSP models typically include coverage-related constraints, and the most commonly occurring constraint in this category is termed "Cover required". Due to the importance of meeting the required number of employees to cover the workload, this constraint is defined as a hard constraint in essentially all PSP formulations that include it, with the exception of the models in [Carter and Lapierre, 2001; Sherali et al., 2002; Topaloglu and Ozkarahan, 2011] (we refer the reader to Appendix B for a detailed account of the constraints defined within each category, and to Appendix $\mathrm{C}$ for a complete assessment of issues pertaining to problem definition and modeling framework). Preference/fairness related constraints are also well addressed in PSP studies, but unlike coverage constraints, they are most commonly defined as soft constraints. The most frequently occurring constraints in this category deal with equally distributing work hours among physicians and residents, and allocating physicians according to their preferred schedule. Finally, some studies, in particular those concerned with resident scheduling, include constraints related to more specific issues, such as professional training in addition to service delivery. 


\subsection{3}

Implementation Issues

A summary of issues related to solution techniques, applicability of the models and instances used in the test phase, and incorporation of uncertainty in studies dealing with PSP is presented in Table 2.6. 
Table 2.6: Implementation issues accounted for in PSP papers

\begin{tabular}{|c|c|c|c|c|c|c|}
\hline \multirow{2}{*}{ Ref. (A) } & \multirow{2}{*}{ Solution technique (B) } & \multicolumn{4}{|c|}{ Applicability (C) } & \multirow{2}{*}{ Uncertainty addressed (D) } \\
\hline & & $\mathrm{A}$ & NAR & NAT & Instances & \\
\hline Bard et al. [2013] & Exact (GP) & - & $\mathrm{x}$ & - & Healthcare provider & - \\
\hline Bard et al. [2014] & Exact (GP/MINP)/Network Optimization & - & $\mathrm{x}$ & - & Healthcare provider & - \\
\hline Bard et al. [2016] & Exact (MINP)/Heuristics & - & $\mathrm{x}$ & - & Healthcare provider & - \\
\hline Baum et al. [2014] & Exact (MIP) & $\mathrm{x}$ & - & - & Healthcare provider & - \\
\hline Beaulieu et al. [2000] & Exact (IP) & - & $\mathrm{x}$ & - & Healthcare provider & - \\
\hline \multirow[t]{2}{*}{ Bowers et al. [2016] } & Exact (MIP) & $\mathrm{x}$ & - & $\mathrm{x}$ & Healthcare provider & - \\
\hline & & - & - & - & + generated data & - \\
\hline Bruni and Detti [2014] & Exact $(\mathrm{MIP} / \mathrm{B} \& \mathrm{C})$ & - & $\mathrm{x}$ & - & Healthcare provider & - \\
\hline Brunner and Edenharter [2011] & Exact (MIP/CG) & - & $\mathrm{x}$ & - & Healthcare provider & - \\
\hline Brunner et al. [2009] & Exact (MIP) & - & $\mathrm{x}$ & - & Healthcare provider & - \\
\hline \multirow[t]{2}{*}{ Brunner et al. [2011] } & Exact $(\mathrm{CG} / \mathrm{B} \& \mathrm{P})$ & - & $\mathrm{x}$ & $\mathrm{x}$ & Healthcare provider & - \\
\hline & & - & - & - & + generated data & - \\
\hline Carrasco $[2010]$ & Metaheuristic (GS)/Heuristics & $\mathrm{x}$ & - & - & Healthcare provider & - \\
\hline Carter and Lapierre [2001] & Metaheuristic (TS) & - & $\mathrm{x}$ & - & Healthcare provider & - \\
\hline Cohn et al. [2009] & Exact (MIP)/Heuristics & $\mathrm{x}$ & - & - & Healthcare provider & - \\
\hline Day et al. [2006] & Exact (MIP) & - & $\mathrm{x}$ & - & Healthcare provider & - \\
\hline Elomri et al. [2015] & Exact (GP) & - & $\mathrm{x}$ & - & Healthcare provider & - \\
\hline Ferrand et al. [2011] & Exact (IP) & $\mathrm{x}$ & - & - & Healthcare provider & - \\
\hline Fügener et al. [2015] & Exact (MIP) & - & $\mathrm{x}$ & - & Healthcare provider & - \\
\hline Gendron et al. [2005] & Exact $(\mathrm{CG}) / \mathrm{CP}$ & - & - & $\mathrm{x}$ & generated data & - \\
\hline Güler [2013] & Exact (GP) & - & $\mathrm{x}$ & - & Healthcare provider & - \\
\hline Güler et al. [2013] & Exact (GP) & $\mathrm{x}$ & - & - & Healthcare provider & - \\
\hline
\end{tabular}




\begin{tabular}{|c|c|c|c|c|c|c|}
\hline \multirow[t]{2}{*}{ Gunawan and Lau [2010] } & Exact (IP)/Metaheuristic (WS/HC/eC) & - & $\mathrm{x}$ & $\mathrm{x}$ & Healthcare provider & - \\
\hline & & - & - & - & + generated data & - \\
\hline \multirow[t]{2}{*}{ Gunawan and Lau [2013] } & Exact (IP)/Heuristics & - & $\mathrm{x}$ & $\mathrm{x}$ & HC provider & - \\
\hline & & - & - & - & + generated data & - \\
\hline Hidri and Labidi [2016] & Exact (IP) & - & $\mathrm{x}$ & - & Healthcare provider & - \\
\hline Huang et al. [2016] & Exact (IP) & - & $\mathrm{x}$ & - & Healthcare provider & - \\
\hline Kazemian et al. [2014] & Exact (IP) & - & $\mathrm{x}$ & - & Healthcare provider & - \\
\hline Lo and Lin [2011] & Metaheuristic (PSO) & $\mathrm{x}$ & - & - & Healthcare provider & - \\
\hline Rosocha et al. [2015] & Metaheuristic (SA) & - & $\mathrm{x}$ & - & Healthcare provider & - \\
\hline Savage et al. [2015] & Exact (MIP) & - & $\mathrm{x}$ & - & Healthcare provider & - \\
\hline Shamia et al. [2015] & Exact (GP) & - & $\mathrm{x}$ & - & Healthcare provider & - \\
\hline Sherali et al. [2002] & Exact (MIP)/Metaheuristic (SH) & - & - & $\mathrm{x}$ & generated data & - \\
\hline Smalley and Keskinocak [2016] & Exact (IP) & - & $\mathrm{x}$ & - & Healthcare provider & - \\
\hline Smalley et al. [2015] & Exact (MIP)/Heuristics & $\mathrm{x}$ & - & - & Healthcare provider & - \\
\hline Stolletz and Brunner [2012] & Exact (MIP) & - & $\mathrm{x}$ & - & Brunner et al. (2009) & - \\
\hline Topaloglu [2006] & Exact (GP) & - & $\mathrm{x}$ & - & Healthcare provider & - \\
\hline \multirow[t]{2}{*}{ Topaloglu [2009] } & Exact (MIP) & - & $\mathrm{x}$ & $\mathrm{x}$ & Healthcare provider & - \\
\hline & & - & - & - & + generated data & - \\
\hline opaloglu and Ozkarahan [2011] & Exact $(\mathrm{MIP} / \mathrm{CG}) / \mathrm{CP}$ & - & $\mathrm{x}$ & - & Healthcare provider & - \\
\hline Turner et al. [2013] & Exact (SP) & - & $\mathrm{x}$ & - & Healthcare provider & $\mathrm{x}$ \\
\hline an Huele and Vanhoucke [2014] & Exact (MIP) & - & $\mathrm{x}$ & - & Healthcare provider & - \\
\hline Wang et al. [2007] & Metaheuristic (GA) & - & - & $\mathrm{x}$ & generated data & - \\
\hline White and White [2003] & Metaheuristic (TS)/CP & $\mathrm{x}$ & - & - & Healthcare provider & - \\
\hline
\end{tabular}


A variety of solution techniques have been employed in PSP studies, as shown in column (B) of Table 2.6. We classify these techniques as exact methods, heuristics, and metaheuristics. Of note, the former is more frequently applied to PSP than the latter two.

With regards to exact methods, the majority of PSP models make use of Mixed Integer Programming (MIP) [Baum et al., 2014; Bowers et al., 2016; Bruni and Detti, 2014; Brunner et al., 2009; Brunner and Edenharter, 2011; Day et al., 2006; Fügener et al., 2015; Sherali et al., 2002; Smalley et al., 2015; Stolletz and Brunner, 2012; Topaloglu, 2009; Topaloglu and Ozkarahan, 2011; Van Huele and Vanhoucke, 2014], Integer Programming (IP) [Beaulieu et al., 2000; Ferrand et al., 2011; Gunawan and Lau, 2010, 2013; Hidri and Labidi, 2016; Huang et al., 2016; Kazemian et al., 2014], or Goal Programming (GP) [Bard et al., 2013, 2014; Elomri et al., 2015; Güler et al., 2013; Shamia et al., 2015; Topaloglu, 2009]. It is worth noting that GP is particularly well suited to the structure of PSP, where staff preferences and individual employment contracts are commonly addressed (in contrast, nurses typically respect collective agreements). To ensure an adequate balance between education and patient care activities, resident scheduling is performed in [Topaloglu, 2009] of an analytical hierarchy process (AHP) based goal programming approach. In a similar application, the assignment of residents and senior academic staff to outpatient clinics is addressed, and AHP is used to incorporate the experiences of the department into a GP model [Güler, 2013]. GP is also used for resident scheduling in Bard et al. [2013] and [Elomri et al., 2015]. In the former, residents must rotate through different clinical experiences monthly and in the latter residents are assigned to two types of shifts in two different departments. Focusing on improved patient care, the authors in [Shamia et al., 2015] propose a GP model to satisfy as many physicians' preferences and duty requirements as possible while ensuring optimum usage of available resources.

PSP studies commonly make use of Column Generation (CG) and Branch-and-Price $(\mathrm{B} \& \mathrm{P})$ techniques to address large instances. In [Brunner and Edenharter, 2011], CG is applied to solve a long-term staffing model for flexible shift scheduling of physicians with different experience levels. The authors in [Brunner et al., 2011] implicitly construct shifts using a MIP model in association with both CG and a B\&P algorithm that applies two different branching strategies to extend the planning horizon of solvable instances. Addressing many aspects of the residency program, a model is proposed in [Topaloglu, 2009] for resident scheduling in which CG is used for solving larger problem instances. Applying constraint programming-based 
CG, two search strategies are proposed in [Gendron et al., 2005] to improve the coordination between master and sub problems. A flexible MIP formulation is given in [Bruni and Detti, 2014] to schedule different medical guard services in hospital departments. To solve this NP-hard problem, a Branch-and-Cut $(\mathrm{B} \& \mathrm{C})$ procedure is used that involves running a branch and bound algorithm and using cutting planes to tighten the linear programming relaxations.

Among the classes of heuristic and metaheuristics algorithms, a variety of different techniques are reported. Emergency room physician scheduling is performed using tabu search in [Beaulieu et al., 2000] and particle swarm optimization in [Lo and Lin, 2011]; both studies consider three non-overlapped shifts and allow user-definable planning horizons. The authors in [Rosocha et al., 2015] focus on optimizing medical staff preferences through a simulated annealing-based algorithm. Resident scheduling is addressed in [Wang et al., 2007], where a genetic algorithm's mutation operator is proposed for cost minimization. A combination of random and greedy strategies with heuristics is reported in [Carrasco, 2010] for assigning guard shifts to physicians. The main issues addressed in this study are strict equity of the workload, regularity of intervals between consecutive shifts, creation of teams with randomly distributed pairings, and long-term scheduling.

Additionally, several studies combine exact methods with heuristic/metaheuristic approaches. PSP in the context of a surgery department is addressed in [Gunawan and Lau, 2010] by incorporating a large number of rostering and resource constraints with physician preferences; for such, three different models are proposed based on the $\epsilon$-constraint and weighted-sum methods, as well as the hill-climbing algorithm. In a subsequent extension of their work, a bi-objective mathematical programming model is used along with a weighted-sum model in which the varying values of weights are calculated by linear interpolation between solutions [Gunawan and Lau, 2013]. The authors in [White and White, 2003] use a constraint programming logic formalism followed by a Tabu Search optimization algorithm to produce a call schedule. The problem of assigning physicians to service and call shifts in a pediatric intensive care unit is addressed in [Smalley et al., 2015], where both a MIP model and an iterative heuristic that incorporates modified versions of the MIP are developed in collaboration with physicians. In [Bard et al., 2016], a MIP model with nonlinear constraints is proposed for annual family medicine block scheduling, and solved using optimization-based heuristics involving relaxations of the original model, post-processing feasibility, and neighborhood search.

Column (C) in Table 2.6 characterizes the approach to model valida- 
tion. A minority of the analyzed PSP studies report having their models implemented in existing healthcare institutions. Some of the distinguishing characteristics of studies in which real-world applications were performed include their ability to account for greater flexibility in meeting the needs of both the organization and physicians, as well as the existence of interaction/collaboration with physicians throughout the modeling process. This not only granted physicians with flexibility in choosing their time-off requests [Ferrand et al., 2011] but also allowed the development of, e.g., schedules that can be modified by the chief resident to accommodate last-minute personnel changes [White and White, 2003].

The vast majority of surveyed PSP formulations do not deal with uncertainty, as shown in Column (D) of Table 2.6. Turner et al. [2013] was the only work to address this issue by incorporating uncertainty in future surgical demand.

\section{3}

\section{Discussion}

Physician scheduling problems deal with a wide range of interesting optimization questions whose answers have practical implications for healthcare providers, patients, and policy makers. Currently, the focus on healthcare staff scheduling is, on one side, on increasing provider efficiency and thus decreasing operational costs, and, on the other side, on reconciling staff preferences and increasing patient satisfaction. Although the trade-off between operational and social aspects is addressed in the literature, several extensions may be suggested to improve formulations. In what follows, we provide suggestions for future research directions in light of the descriptive dimensions identified in our analysis.

Problem definition: The main shortcoming of existing PSP models in this regard has to do with limited flexibility of current problem settings. In effect, the majority of surveyed studies consider fixed values of planning horizon, number, length, start and overlap of shifts, and substitutability/number of skill categories. Moreover, no PSP studies were found in which the user is allowed to define substitutions and/or the number of skill categories. In this context, allowing the user to input meaningful case-specific settings is the first step towards building more realistic staff scheduling formulations. Additional settings, such as the availability of shared staff between different hospital departments, could also be taken into account. Hence, future research on increasing model flexibility is required and should be undertaken as a collaborative effort between algorithm developers and end users from healthcare institutions. 
Modeling framework: The current trend in physician scheduling is to account for staff preferences in the problem formulation. This points to the importance of uncovering which aspects of the work schedule are most relevant to healthcare staff, to ensure that these will be adequately addressed in ensuing formulations. Future research would thus profit from incorporating findings from social science studies dealing with employee needs/priorities. Of note, due to the complexity of accounting for staff preferences when dealing with nonresident scheduling, existing PSP models seldom do so, and there is much room for developing models that address such important distinguishing features. Furthermore, most of the surveyed studies disregard issues related to staffing (i.e., determining the number of required personnel with a given set of skills) and assume the number of staff members for each day and shift to be previously known. This limitation could be addressed by extending the existing models to capture practical assumptions such as uncertainty of demand and multitype demand (e.g., variable patient arrival rates, different classes of patients depending on the type of required care, etc.). The inclusion of constraints referring to mandatory breaks, e.g., lunch breaks, would also lead to more realistic models. Examples of considerations relative to mandatory breaks include what time such breaks should be taken; maximum cumulative time without a break; how to split breaks to better distribute them over the working day, among others. Finally, new formulations and/or model extensions should include stochastic and dynamic conditions that account for variations in the problem setting over time, e.g., significant changes in management objectives and patient population characteristics.

Implementation issues: Exact methods have had limited success in dealing with the enormous search space of real staff scheduling problems, and for this reason, a prominent line of research focuses on hybridizing exact and heuristic approaches. Recent algorithmic advances warrant further investigation into novel combinations of existing methods and improvements upon existing combinations. Alternatively, continued research is required into improved applications of exact methods to large-sized instances. Also of interest is the incorporation of robust and efficient solution methods in decision support systems, which would allow for real-time evaluation of a variety of scheduling scenarios. Finally, addressing more realistic real-world applications in an uncertain dynamic setting calls for future research into multi-stage uncertain programming, such as multi-stage stochastic programming, stochastic dynamic programming, or adjustable robust optimization approaches.

A minority of surveyed studies takes into account factors such as the unpredictable presence of workers due to illness, arrival delays, demand uncer- 
tainty, uncertainty in task duration, and patient-related uncertainties. Hence, future research on stochastic nurse and physician scheduling is required. In addition to investigating how to incorporate uncertainty into PSP models, improved applications of stochastic simulation of demand patterns and staff availability could be devised to test the robustness of deterministic approaches. Another poorly addressed facet of healthcare staff scheduling has to do with the issue of the rescheduling (or rerostering). Further analysis is warranted on coping with post hoc reevaluation of existing schedules due to, e.g., the existence of staff members who become unable to perform assigned tasks.

Most of the reviewed PSP formulations offer simplistic representations of practical settings that are not directly applicable to real healthcare institutions. Hence, there is a gap between the current literature and the required necessities of existing hospital environments that must be addressed by future research. Moreover, in cases when real data is used to generate and validate scheduling models, implementation details are scarcely provided. Hence, increased disclosure will play a crucial role in promoting the successful employment of healthcare staff scheduling algorithms in real-world settings. Finally, comparisons between similar or competing approaches are a challenging task due to the intrinsic variability of problem formulations accruing from the plethora of national and regulatory settings in existence worldwide. To address the issue of replication and equivalence of solution, benchmark test problems for NSP and PSP need to be made publicly available.

Additional related issues: Further research into several associated topics would also serve to leverage improvements in healthcare staff scheduling solution methods. For one, the development of predictive models for demand forecasting could lead to PSP formulations that are more robust to planning period specifications. Additionally, further research is needed in designing statistical methods to estimate the input parameters of existing models. Another issue that warrants consideration relates to integrating quality of care metrics and other strategic, tactical, and operational decisions with scheduling arrangements. Finally, further work is required on incorporating engineering economic models into the design phase of multi-period models, and on accounting for budget constraints and various types of costs related to the healthcare system and its clients.

\section{4}

\section{Conclusions}

In this chapter, we surveyed literature dealing with the physician scheduling and identified a lack of updated reviews on the subject. We addressed this 
shortcoming by providing a background of the problems of physician scheduling. A comprehensive framework for classifying such problems was introduced that focused on three descriptive dimensions of healthcare staff scheduling, namely problem definition, modeling framework, and implementation issues. PSP formulations were found to be similar with regards to the limited flexibility of current problem settings. Additionally, we recognized a current trend in accounting for staff preferences within both formulations and noted the scarcity of PSP models dealing with stochastic and dynamic conditions. Physician scheduling formulations were also analyzed in terms of a variety of implementation issues and other associated topics, whereby it was possible to suggest directions for future research.

In this Thesis, we addressed several aspects pointed in the previous Section as trends and research directions. In the models proposed in the course of the Thesis, we include different settings to increase model flexibility and to address more realistic applications, e.g. we consider different shift lengths, overlapped shifts, flexibility in the shift's start time, different skill categories, and we also considered cyclic and acyclic approaches to perform staff schedule in the models presented in Chapters 4 and 5 . We treated physicians individually to account for preferences and/or restrictions such as start time allowed for each physician, weekend scheduling rules, availability of each physician. Finally, in the models presented in Chapter 4, 5 and 6, we addressed uncertainty in the demand once the vast majority of the works do not deal with this important feature, as pointed before. 


\section{Methods}

In this chapter we discuss the Sample Average Approximation (SAA) to deal with the uncertainty. Moreover, we present a solution framework adopted in the thesis showing the methodology steps adopted in this thesis to deal with uncertainty.

\section{1}

\section{Dealing with Demand Uncertainty Using Sample Average Approximation}

To take into account uncertainty in the course of this thesis, we assume that uncertainty is discretely represented by $S C$ possible realization scenarios. However, the number of possible realization scenarios for a given probability distribution is infinite, thus it is not computationally feasible to consider all of the possible scenarios. To circumvent this difficulty, we can use sampling techniques that consider a random subset of possible realizations to obtain approximate results.

The Sample Average Approximation (SAA) is a technique proposed by Shapiro and Homem-de Mello [1998]. The main idea of this technique is to approximate the value of the objective function considering the average value of the successive solution to several problems composed of subsets of scenarios sampled successively. The resulting sample average approximation of the stochastic program is then solved by some appropriate deterministic optimization procedure. The process must be repeated with different samples to obtain candidate solutions along with statistical estimates of their optimality gaps.

SAA has been widely used in the literature to avoid dealing with a large number of scenarios but still treating uncertainty adequately. The papers of [Kleywegt et al., 2002; Linderoth et al., 2006; Mak et al., 1999] presented important theoretical considerations regarding the method. We see some applications of SAA in scheduling problems in healthcare, for example: to generate an optimal surgery schedule of elective surgery patients with uncertainty both in surgery durations and availability of downstream resources [Min and Yih, 2010]; to perform nurse scheduling taking into account uncertainties in demand and length of stay of patients over time [Bagheri et al., 2016]; and to perform 
physician staffing in an Emergency Department considering uncertainties in patient arrival and service times [Daldoul et al., 2018; EL-Rifai et al., 2015].

In this chapter, we present the development of SAA techniques to deal with uncertainty. In this thesis, more specifically, uncertainty related to patient arrivals in an Emergency Department considered in the model presented in Chapter 4 and 5 and the patient referred to a specialty surgical unit considered in the model presented in Chapter 6. We show how to approximate the solution by means of statistical bounds to be obtained by repeatedly solving the problem considering samples from the original scenario set. We used two methods of scenario generation to represent the uncertainty addressed. Moreover, we show techniques to evaluate the stability of the scenario generation methods to establish the best option between these methods; and thus determine the number of suitable scenarios to solve the problem accurately.

\subsection{1}

\section{Sample Average Approximaton}

We consider scenario-based optimization problems represented of the form:

$$
v^{*}=\min _{x \in X}\{f(x)=\mathbb{E} F(x, \xi)\}
$$

Here $\xi$ is a random vector whose distribution is assumed known, $X$ is a infinite set, $F(x, \xi)$ is a real valued function of two (vector) variables $x$ and $\xi$, and $\left.\mathbb{E} F(x, \xi)=\int_{\Omega} F(x, \xi) P(d \xi)\right\}$ is the corresponding expected value.

In realistic applications, the main concern about solving the problem in $(3-1)$ is related to the calculation of $\mathbb{E} F(x, \xi)$ due to the large dimensionality of $\xi$. For this, the basic idea of the SAA approach proposed in [Shapiro and Homem-de Mello, 1998] is that a sample of $N$ independent replications of the random vector $\xi$ is generated and consequently the expected value function is approximated by the average function:

$$
\hat{v}_{N}=\min _{x \in X}\left\{\tilde{f}_{N}(x)=\frac{1}{N} \sum_{n=1, \ldots, N} F\left(x, \xi^{N}\right)\right\}
$$

The function $\tilde{f}_{N}$ is a sample-average approximation (SAA) to the objective $f$ of $(3-1)$. The value of $\hat{v}_{N}$ is random once it is a function of the corresponding random sample. However, for a particular realization $\xi_{1}, \ldots, \xi_{N}$, the problem (3-2) is deterministic and, thus, can be solved by some appropriate deterministic optimization procedure.

The SAA problem (3-2) has two important properties showed in [Mak et al., 1999]: 
Property 1. $\tilde{f}_{N}(x)$ is an unbiased estimator for $f(x)$.

Property 2. $\hat{v}_{N}$ is a lower bound for $v^{*}$.

Linderoth et al. [2006] showed that, using the SAA approach and the ideas introduced by Norkin et al. [1998] and Mak et al. [1999], it is possible to obtain upper and lower bounds for the optimal value of the real problem and, furthermore, that such boundaries converge to the true solution with probability 1 as $N$ increases. The ideas showed by Linderoth et al. [2006] are presented in Sections 3.1.1.1, 3.1.1.2 and 3.1.1.3 as follows.

\subsubsection{1}

\section{Lower bound estimates}

The expected value $\mathbb{E} \hat{v}_{N}$ can be estimated as follows. We generate $M$ independent samples, each sample with $\mathrm{N}$ replications, $\xi_{n m}, n=1, \ldots N ; m=$ $1, \ldots M$, and we solve the corresponding SAA problem:

$$
\hat{v}_{N}^{m}=\min _{x \in X}\left\{\frac{1}{N} \sum_{n=1, \ldots, N} F\left(x, \xi^{n m}\right)\right\}
$$

Therefore, we can compute an approximation for $\mathbb{E} \hat{v}_{N}$ as:

$$
L_{N M}=\frac{1}{M} \sum_{m=1, \ldots, M} \hat{v}_{N}^{m}
$$

According to Property 1 , the estimate $L_{N M}$ is an unbiased estimator to $\mathbb{E}\left[\hat{v}_{N}\right]$ and therefore, provides a statiscal lower bound for the true optimal value of $v^{*}$ in (3-1) [Linderoth et al., 2006; Mak et al., 1999].

We have by the Central Limit Theorem that

$$
\sqrt{M}\left[L_{N M}-\mathbb{E}\left[\hat{v}_{N}\right] \Rightarrow N\left(0, \sigma_{L}^{2}\right), \quad \text { as } M \rightarrow \infty,\right.
$$

where $\sigma_{L}^{2}=\operatorname{Var}\left[\hat{v}_{N}\right]$ and " $\Rightarrow$ " denotes convergence in distribution. To approximate $\sigma_{L}^{2}$, we can used the sample variance estimator $s_{L}^{2}(M)$, which is defined as

$$
s_{L}^{2}(M)=\frac{1}{M-1} \sum_{m=1, \ldots, M}\left(\hat{v}_{N}^{m}-L_{N M}\right)^{2} .
$$

Defining $z_{\alpha}$ as the standard normal deviate such that $P\left(z \leq z_{\alpha}\right)=1-\alpha$, and using the approximation $s_{L}^{2}(M)$ to $\sigma_{L}^{2}$, we can define a $(1-\alpha) \%$ confidence interval for $L_{N M}$ as

$$
\left[L_{N M}-\frac{z_{\frac{\alpha}{2}} s_{L}(M)}{\sqrt{M}}, L_{N M}+\frac{z_{\frac{\alpha}{2}} s_{L}(M)}{\sqrt{M}}\right] .
$$




\subsubsection{2}

\section{Upper bound estimates}

An upper bound can be obtained by noting that for any $\hat{x} \in X$, we have immediately from (3-1) that $f(\hat{x}) \geq v^{*}$. Therefore, by selecting $\hat{x}$ to be a nearoptimal solution (possible using the SAA problem (3-2)), and by using some unbiased estimator of $f(\hat{x})$, we can obtain an estimate of an upper bound for $v^{*}$. To obtain such an estimate, we generate $T$ independent batches of samples of size $\bar{N}$, denoted by $\xi_{n t}, n=1, \ldots, \bar{N} ; t=1, \ldots, T$, and we define

$$
\mathbb{E}\left[\hat{f}_{\bar{N}}^{t}(x)=\frac{1}{\bar{N}} \sum_{n=1, \ldots, \bar{N}} F\left(\hat{x}, \xi_{n t}\right)\right]=f(x), \quad \forall x \in X .
$$

We can then use the average value defined by

$$
U_{\bar{N}, T}(\hat{x})=\frac{1}{T} \sum_{t=1, \ldots, T} \hat{f}_{\bar{N}}^{t}(\hat{x})
$$

as an estimate of $f(\hat{x})$. By applying the Central Limit Theorem again, we have that

$$
\sqrt{T}\left[U_{\bar{N}, T}(\hat{x})-f(\hat{x})\right] \Rightarrow N\left(0, \sigma_{U}^{2}(\hat{x})\right), \quad \text { as } T \rightarrow \infty,
$$

where $\sigma_{U}^{2}(\hat{x})=\operatorname{Var}\left[\hat{f}_{\bar{N}}^{t}(\hat{x})\right]$ and " $\Rightarrow$ " denotes denotes distributional convergence to a normal distribution. We can replace $\sigma_{U}^{2}(\hat{x})$ by the sample variance estimator $s_{U}^{2}(\hat{x}, T)$, defined by

$$
s_{U}^{2}(\hat{x}, T)=\frac{1}{T-1} \sum_{t=1, \ldots, T}\left(\hat{f}_{\bar{N}}^{t}(\hat{x})-U_{\bar{N}, T}(\hat{x})\right)^{2} .
$$

By replacing $\sigma_{U}^{2}(\hat{x})$ with $s_{U}^{2}(\hat{x}, T)$, we can proceed as to Lower Bound estimation to obtain a $(1-\alpha) \%$ confidence interval for $f(\hat{x})$ :

$$
\left[U_{\bar{N}, T}(\hat{x})-\frac{z_{\frac{\alpha}{2}} s_{U}(\hat{x}, T)}{\sqrt{T}}, U_{\bar{N}, T}(\hat{x})+\frac{z_{\frac{\alpha}{2}} s_{U}(\hat{x}, T)}{\sqrt{T}}\right] .
$$

Kleywegt et al. [2002] highlights that an important question is how the choice about the number of samples should be made to estimate both lower bound and upper bound. The authors affirm that with larger $N$, the objective function of the SAA problem (3-2) tends to be a more precise approximate of the true objective function. However, the computational complexity for solving the SAA problem (3-2) increases at least linearly, and often exponentially, in the sample size $N$. According to the same authors, although computational complexity motivate to choose a small sample size $N$ for the SAA problem, it makes sense to choose a larger sample size $\bar{N}$. Since in this case the evaluation of $f$ provided a fixed solution $\hat{x}$ is not computationally demanding and can also take advantage from techniques such as decomposition and parallelization. Another issue addressed by Kleywegt et al. [2002] is the choice of the number 
of replications $M$ for the lower bound estimates and $T$ for the upper bound estimates. They advocate that this choice, like the choice of sample size $N$ and $\hat{x}$, may be done dynamically.In related to this topic, Bayraksan and Morton [2006] assessing solution quality in stochastic programs, they advocate that quality is defined via the optimality gap and they proposed a procedure which the output is the confidence interval on this gap. Thus, they showed how to use a small number of replications can be used to make a valid statistical inference on the quality of a candidate solution.

\subsubsection{3}

\section{Estimating the Gap}

We may wish to estimate the optimality gap $f(\hat{x})-v^{*}$. Provided estimates in (3-4) and (3-9) for the lower bound and upper bound, respectively, we can consider the gap as the difference

$$
\operatorname{Gap}_{N, M, \bar{N}, T}(\hat{x})=U_{\bar{N}, T}(\hat{x})-L_{N, M} .
$$

By the Law of Large Numbers we have that $\operatorname{Gap}_{N, M, \bar{N}, T}(\hat{x})$ converges to $f(\hat{x})-v^{*}$ with probability one as $N, M, \bar{N}$, and $T$ tend to $\infty$. The variance $s_{G A P}^{2}$ of $\operatorname{Gap}_{N, M, \bar{N}, T}(\hat{x})$ is the estimated by

$$
s_{G A P}^{2}=s_{U}^{2}(\hat{x}, T)+s_{L}^{2}(M)
$$

Of note, three factors contribute to the error in the statistical estimator $\operatorname{Gap}_{N, M, \bar{N}, T}(\hat{x})$ of the gap $f(\hat{x})-v^{*}$, that are:

(1) variance of $U_{\bar{N}, T}(\hat{x})$;

(2) variance of $L_{N, M}$;

(3) bias $v_{*}-\mathbb{E}\left[\hat{v}_{N}\right]$.

Recall that $U_{\bar{N}, T}(\hat{x})$ and $L_{N, M}$ are unbiased estimators of $f(\hat{x})$ and $\mathbb{E}\left[\hat{v}_{N}\right]$, respectively [Linderoth et al., 2006; Mak et al., 1999]. We have here that $\operatorname{Gap}_{N, M, \bar{N}, T}(\hat{x})$ is an unbiased estimator of $f(\hat{x})-\mathbb{E}\left[\hat{v}_{N}\right]$, and that $f(\hat{x})-\mathbb{E}\left[\hat{v}_{N}\right] \geq$ $f(\hat{x})-v^{*}$. That is, $\operatorname{Gap}_{N, M, \bar{N}, T}(\hat{x})$ overestimates the true gap $f(\hat{x})-v^{*}$, and has bias $v^{*}-\mathbb{E}\left[\hat{v}_{N}\right]$.

In addition, Linderoth et al. [2006] expose that for ill-conditioned problems, this bias may be relatively large and tends to zero at a rate of $O\left(N^{-1 / 2}\right)$; the bias can be reduced by increasing the sample size $N$ of the corresponding SAA problems or by sampling more intelligently (e.g., using LHS); an increase in $N$ leads to a larger problem instance to be solved, while increases in $\bar{N}, M$ and $T$ to reduce factors (1) and (2) that contribute to the error lead only to more instances of the same size to be solved. 


\subsection{2}

\section{Scenario Generation Methods}

Several sampling techniques can be used to generate scenarios for stochastic optimization problems. The Monte Carlo Sampling method is the most commonly used with SAA since the technique was originally proposed using this type of sampling. However, as mentioned above, the bias can be reduced by sampling using another method. Following is a brief description of two sampling methods, from the several available in the literature, that we used in this study.

\subsubsection{1}

\section{Monte Carlo Sampling}

Monte Carlo Sampling (MCS) provide approximate solutions to a variety of mathematical problems by performing statistical sampling experiments [Pengelly, 2002]. This technique has formally existed since the early 1940s, when it was applied in nuclear fusion research.

At the heart of any Monte Carlo method is a random number generator which is a procedure that produces an infinite sample of random variables that are independent and identically distributed (iid) according to some probability distribution [Kroese et al., 2013].

According to Kroese et al. [2013], most computer languages already contain a built-in random number generator. Hence, it is only requested the input of an initial number, called the seed, and upon invocation, the random number generator produces a sequence of independent random uniform variables on the interval $(0,1)$. The vast majority of current random number generators are based on simple algorithms that produce a sequence $U_{1}, U_{2}, U_{3}, \ldots$ of pseudorandom numbers. They are called pseudorandom numbers because once starting from a certain seed, the sequence of random numbers must repeat itself since the state space is finite.

The convergence rate associated with the MCS is $O(1 / \sqrt{K})$, i.e., the speed at estimator error decreases as the sample size $K$ increase [Glasserman, 2013].

\subsubsection{2}

\section{Latin Hypercube Sampling}

A large number of samples are typically required in traditional MCS to achieve good precision; for this reason, various techniques exist to improve MCS accuracy. Latin Hypercube Sampling (LHS) is one of these techniques widely-used to generate controlled random samples. Firestone et al. [1997] 
states that LHS is considered to be more efficient than MCS since it requires fewer simulations to produce the same level of precision, and it is generally recommended when the model is complex or when time and resource constraints are an issue.

LHS constructs a highly dependent joint probability density function for the random variables in the problem, which allows good precision in the response parameters using a small number of samples [Huntington and Lyrintzis, 1998]. In this way, LHS operates by dividing the subspace of each vector component $s_{i} ; i=1, \ldots, N$ into $M=n$ disjoint subsets (strata) of equal probability $\Omega_{i k} ; i=1, \ldots, N ; k=1, \ldots, M$. Samples of each vector component are drawn from the respective strata according to

$$
x_{i k}=D_{x_{i}}^{-1}\left(U_{i k}\right) ; i=1, \ldots, N ; k=1, \ldots, M
$$

where $U_{i k}$ are iid uniformly distributed samples on $\left[\xi_{k}^{l}, \xi_{k}^{u}\right]$ with $\xi_{k}^{l}=(k-1) / M$ and $\xi_{k}^{u}=(k / M)$ [Shields and Zhang, 2016]. Thus, the samples $x_{i k}$ is randomly selected from each vector component (without replacement), these terms are grouped to produce a sample, and this process is repeated $M=n$ times.

\subsection{3}

\section{Evaluation of Scenario Generation's Methods}

We discussed in Section 3.1.1 that, due to the difficulty of solving the original problem, we replace it with the scenario-based problem (3-1). As a result, we solve only an approximation of 3.1.1, and the quality of the approximation is directly linked to the quality of the scenario tree [Kaut and Wallace, 2007].

Kaut and Wallace [2007] outline that different scenario generation methods represent the relationships among the data in different ways, and hence end up with different trees. In this manner, the authors focus on the evaluation of a given scenario generation method instead of trying to find the optimal method. Therefore, they proposed the stability tests in-sample and out-of-sample that allow users who already have one or more scenario generation methods implemented to test how they perform for a given optimization problem.

Stability tests are based on SAA and used to determine the number of scenarios required to solve a stochastic optimization problem accurately. The in-sample and out-of-sample stability tests are defined below. 


\subsubsection{1}

\section{In-sample stability}

In-sample stability condition guarantees that for every scenario tree chosen, the optimal value of the objective function reported by the model should be (approximately) the same. Thus, if to solve a stochastic programming model we perform $\mathrm{L}$ replications of an independent sample $\xi_{l}, l=1, \ldots, L$ with $K$ scenarios generated from a probability distribution of an uncertain parameter $\xi$ we have:

$$
F\left(x_{l}^{*}, \xi_{l}\right) \approx F\left(x_{l^{\prime}}^{*}, \xi_{l^{\prime}}\right) \quad l, l^{\prime} \in 1, \ldots, L
$$

where $x_{l}^{*}$ is the optimal first-stage solution of replication $l$.

\subsection{4}

\section{Out-of-sample stability}

The out-of-sample condition states that solving the stochastic programming problem for each scenario tree, the optimal first-stage solution $x_{l}^{*}$ of each replication $l$ should get (approximately) the same value in the objective function when evaluated with the true distribution of the uncertain parameter $\xi$, i.e.,

$$
F\left(x_{l}^{*}, \xi\right) \approx F\left(x_{l^{\prime}}^{*}, \xi\right) \quad l, l^{\prime} \in 1, \ldots, L .
$$

The problem with this definition of stability is that we can test it only if we can evaluate the true objective function and for this, it is necessary to know completely the distribution of the uncertain parameter. In such cases, one option is to define a set of reference scenarios large enough to approximate the true distribution.

\section{2}

\section{Solution framework}

The solution framework we propose is shown in Figure 3.1. Our solution framework is divided into two environments. The real data environment, where we have the real data from the case studies addressed. These data is processed, cleaned and used in the study environment. The study environment is where we no longer work with real data but with inferred statistical distributions from the data, scenarios generated by the methods presented above and simulated data.

The first step in our solution framework is to perform a statistical analysis to fit patient arrival data to known probability distributions, and then generate scenarios using these distributions. In the second step, we run preliminary tests 
using Sample Average Approximation and the optimization model to define the settings (number of scenarios and scenario generation method) to be used for each case study of each problem. The third step consists of using the settings from Step 2 to generate the recommended schedule for each case study. In the last step, we use a stochastic discrete-event simulation model to evaluate the performance of the recommended schedule, using the settings from Step 2.

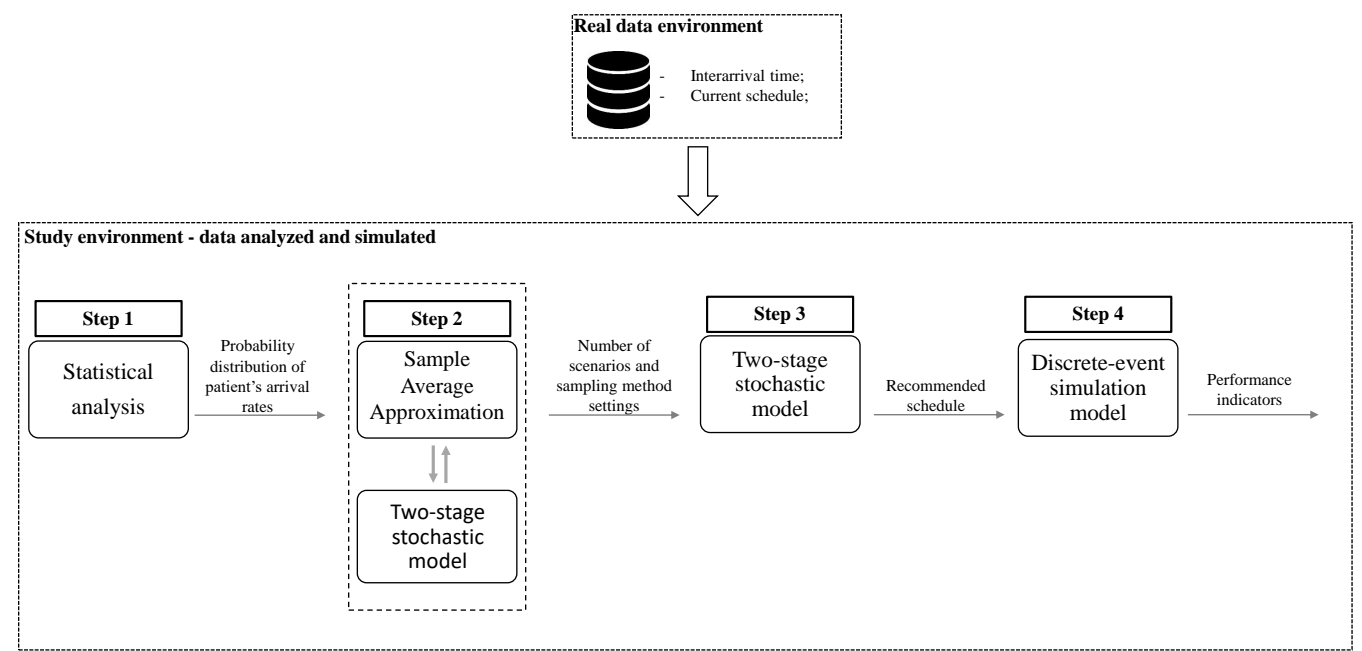

Figure 3.1: Solution framework

\subsection{1}

\section{Statistical analysis}

To account for uncertainty in demand, we fit a distribution for the patients arrival patterns. With the probability distribution that represent the patients arrival, we generate the scenarios used in the SAA. Each scenario representing a possible demand curve for the planning horizon considered in the problem. We also used the probability distributions of the patients arrival in the simulation model.

We can then write that:

$$
D E M_{h t}^{c}=f\left(\lambda_{h t}\right)
$$

where $D E M$ is the demand per hour $h$, period $t$, and scenario $c$ and parameter $\lambda_{h t}$ represents the average number of patients who arrive at the ED during each hour $h$, and period $t$. 


\subsection{2}

\section{Methodology using Sample average approximation to deal with uncer- tainty in the thesis}

By modeling patient arrival using the probability distributions from Section 3.2.1, an infinite number of possible realization scenarios could be generated. Since it is not computationally feasible to consider all possible scenarios of demand levels we use Sample Average Approximation (SAA) to circumvent this difficulty as was discussed in Section 3.1.

The procedure for incorporating SAA in our numerical experiments is shown in Algorithm 1, with the following parameters: the number of replications $M$ for lower bound calculation; the initial number of scenarios $N$ for lower bound calculation; the number of replications $T$ for upper bound calculation; the number of scenarios $\bar{N}$ for upper bound calculation; the stop criterion $\epsilon$ which is the difference of $1 \%$ between the lower and upper bounds (i.e. an estimated gap of $1 \%$ ) with $95 \%$ level of confidence interval (CI); $\delta$ which is the increment for the number of scenarios $N$. The parameters received by the procedure may change according to the problems addressed. Then, we use Algorithm 1 to run preliminary tests that allow us to define our optimization model settings which means define the sampling methodology and number of scenarios to be used in each case study.

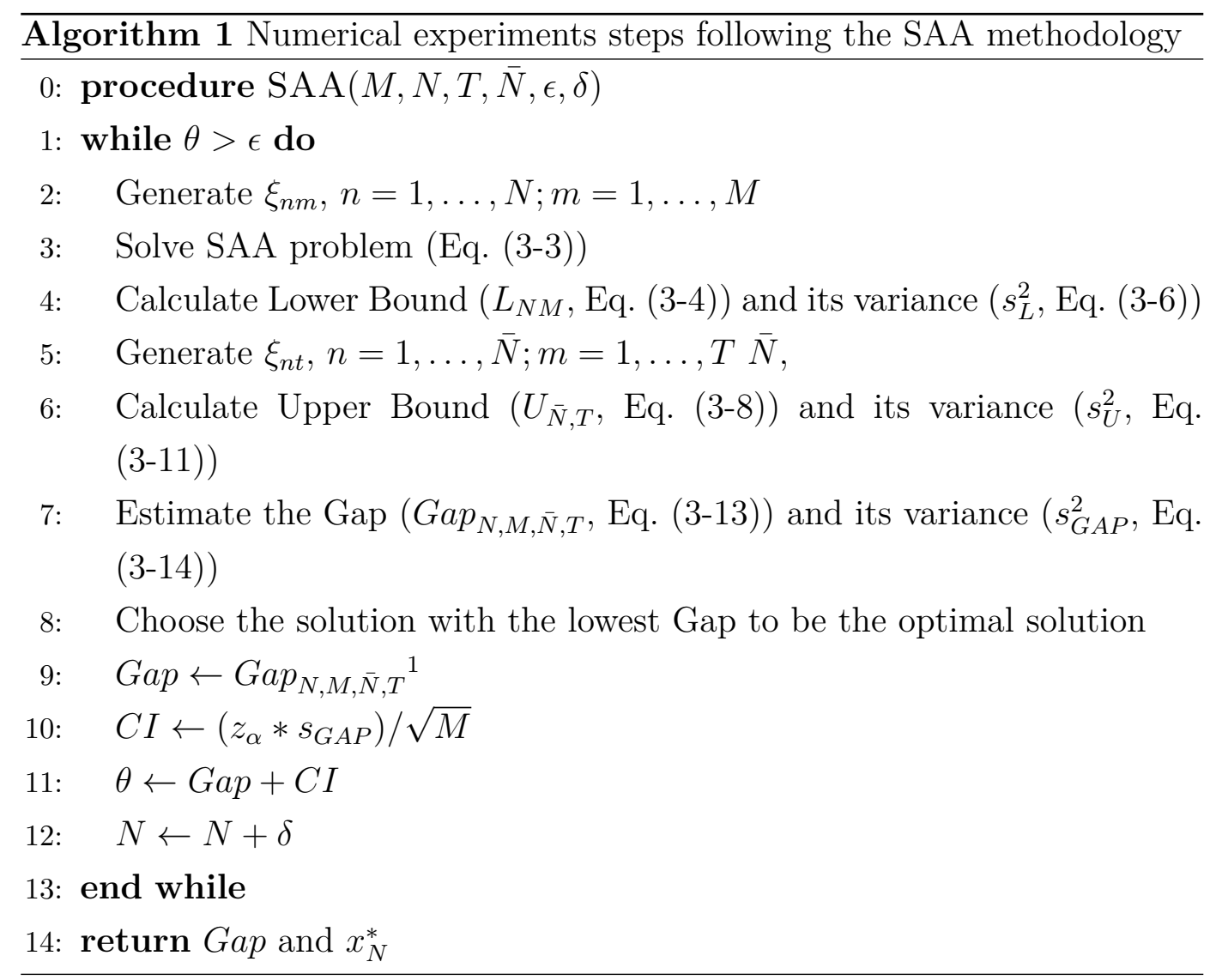


$\mathrm{a}_{\text {The }} \operatorname{Gap}_{N, M, \bar{N}, T}$ is the lowest Gap

\subsection{3}

\section{Two-stage stochastic programming formulation}

The optimization model is based on the two-stage stochastic programming formulation. In the course of the thesis, we propose three models once we addressed three different problems, each model is related to one problem. These models are detailed in the chapter related to each problem. Uncertainties in the model are related to the levels of demand (patients arrivals in the hospital), and we assume that uncertainties are discretely represented by a number of possible realization scenarios (generated in the Step 1). In the optimization model, we assume equal probability of occurrence for the scenarios used.

\subsection{4}

\section{Discrete-event simulation model}

We use discrete-event simulation to compare the results from the optimization model proposed with the results using the current configuration adopted in the hospital studied, estimating the benefits in terms of patient queue frequency, number of patient waiting, and waiting time. Since the simulation model is in the study enviroment, the results related to the current configuration is also a simulated result. This means that we input in the simulation model the current configuration of the hospital and take as output the indicators to this configuration. Then, we compare the indicators achieved when run the results from the proposed model with the indicators achieved when run the current configutation.

For simulation purposes, we use the distribution probability fit as explained in Section 3.2.1, and we assume an exponentially distributed interservice times (as assumed in [EL-Rifai et al., 2015]). Moreover, we are working with a system with multiple available servers.

\section{3}

\section{Conclusions}

In this chapter, we presented the methodology using the SAA to deal with the uncertainty that we will consider in the following chapters. Moreover, we present two scenario generation methods to test in the problems addressed which method converges faster in terms of number of scenarios required to statistically guarantee that the solutions obtained are good. 
In the following chapter, we will propose a model formulation to the 'Physician Staffing and Scheduling for Reducing Door-to-Doctor Time in an Emergency Department' problem based on two-stage stochastic programming. Moreover, we will present the results for two Brazilian case studies using both historical data as scenarios and results using scenario generation based on the SAA technique presented in this chapter. 


\section{4}

\section{Physician Staffing and Scheduling for Reducing Door-to- Doctor Time in an Emergency Department}

In this chapter, we present a description and the specific characteristics of the physician staffing and scheduling problem in an emergency department focusing on reducing door-to-doctor time. In the sequence, we propose the mathematical model formulated to represent the problem. Finally, we present the results of two real case studies on Brazilian hospitals.

\section{1}

\section{Problem Description}

The problem in question can be defined as the Physician Staffing and Scheduling in an Emergency Department (PSSED), where one seeks to determine the number of physicians required to be in service during each shift, for each day of the planning horizon, but also assigns individual physicians to each shift in accordance with service requirements and contractual agreements, taking into consideration uncertainty in patient arrival patterns. Such decisions arise in the context of tactical planning faced by hospitals. We consider this problem as an integrated model able to perform both staffing and scheduling phases together.

Typically, the patient flow through the Emergency Department (ED) is represented as a queuing system consisting of treatment steps and the resources needed. The process begins when patients arrive in the ED. Usually, the patients go to a nurse (to the triage) who determine whether they need vital medical care. If the patients do not need vital care, they stay in a waiting room until a physician is available to do the first assessment. After the first assessment, the patients may follow different paths varying from case to case and depending on how each case is treated in each ED.

Figure 4.1 represents a small example of patient flow in the ED, which is distinct per day and hour. Patients in the system wait in a queue until they are served for one of the servers. Here we are concerned about first assessment, once the door-to-doctor time is an important ED performance measure [Welch et al., 2006]. Therefore, we are interested in performing the staffing and scheduling for the physicians to be placed in this stage of service in the ED so that the 
door-to-doctor waiting time is minimized.

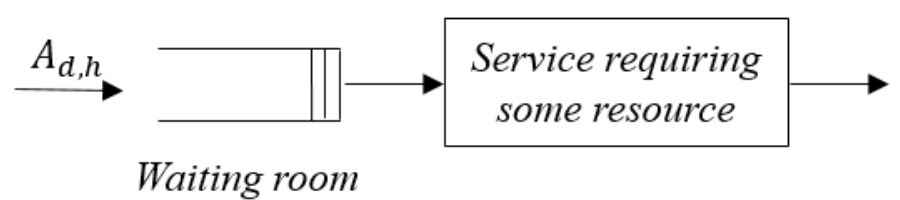

Figure 4.1: Simplified queue model that represents the process in the ED

The PSSDE is performed for a planning horizon that consists of a set of subsequent days of finite and fixed length $D$. In our model, the planning horizon can be divided into $T$ periods over which the staffing level will be repeated (we explain this feature in more detail later). We assume that a fixed number of physicians $P$, belonging to $K$ different skill categories, must be scheduled over the planning horizon. Each day in the planning horizon consists of $H$ hours and is divided in $S$ shifts that may overlap. Moreover, each shift is associated with a specific number of hours, defined in terms of the shift's start time and duration. It is required that a minimum number of physicians is always available in the ED. Each physician is scheduled for a certain number of hours during the entire planning horizon, but distinguish between weekday and weekend shifts. The physician scheduling should respect a minimum interval between assignments. Eventually, when the physician is assigned for the night shift, the interval between assignments should be higher than when the physician is assigned to other shifts. Furthermore, physician scheduling should respect the maximum allowed consecutive hours of work.

The patient's arrival varies per day and hour and meeting appropriately the patient's arrival (that here is the demand) is important in the present context. Therefore, the uncertainty of the demand must be considered into the model so that the decisions are properly taken, considering different possibilities for these uncertain events.

To address this issue, we propose a two-stage stochastic programming formulation with fixed recourse. The mathematical model's objective is to guarantee that enough physicians will be available to meet patient demand, to minimize the total number of patients waiting per hour while taking into account scheduling requirements and contractual agreement constraints.

The first-stage decisions (assumed to be made before the realization of random variables, i.e., previously to the unveiling of the uncertainties) define how many physicians are required and the physicians to be assigned to each day and shift in the planning horizon. Integer and binary variables, respectively represent these decisions. 
Typically, these decisions are taken for one month. Given that the focus of the model is to define the ideal number of physicians for each day and shift, considering the available physicians for the ED operations, and schedule them, aspects related to the strategic level, as number of physicians to be hired to the $\mathrm{ED}$ are not considered as objects of this decision model but as aspects that have already been decided in advance. However, the result of the proposed model can be a good indicative if the total number of doctors available for work in the ED is appropriate.

Second-stage decisions are taken under complete information of the uncertainty. We assume that uncertainties (patients arrivals) are discretely represented by $S C$ possible realization scenarios. We further assume that the probability of scenario $c$ occurring is represented by a scenario-dependent parameter $P R O B_{c}$, defined such that $P R O B_{c} \geq 0$ and $\sum_{c=1}^{S C} P R O B_{c}=1$. We assume that the scenarios have the same probability of occurrence.

To propose the mathematical model, we presume the system is initially empty. For this, we start the scheduling horizon at an hour when the system is usually empty.

The literature on staffing and scheduling in EDs seldom accounts for uncertainty in patient arrivals. Hung et al. [2007] incorporated stochastic demand patterns into a Physician Scheduling Analysis Tool (PSAT) designed to assist in physician staffing and use discrete-event simulation to test Pediatric ED staffing scenarios. A simulation-optimization approach that explores solutions iteratively was developed by Kuo [2014]. In Savage et al. [2015], a mixed integer programming model was proposed to optimize ED physician staffing based on the historical patient arrival data. More recently, Vile et al. [2016] introduced a Decision Support System (DSS) that generates future demand predictions through Singular Spectrum Analysis, suggests minimum staffing requirements based on queueing theory and creates low-cost schedules through linear programming.

Our work makes contributions in both a conceptual and practical level. From a conceptual perspective, we propose a generalizable and integrated model, which simultaneously accounts for ED physician staffing and scheduling problems and, is in contrast with the majority of existing work, where staffing and scheduling are treated as separate problems, neglecting the intrinsic interaction between them [Hung et al., 2007; Savage et al., 2015; Vile et al., 2016]. In fact, when staffing and scheduling are performed separately, the scheduling phase may become infeasible, since service requirements and contractual agreements may render impractical finding a scheduling that meets the established staffing levels. By performing the two steps in an integrated manner, it 
is possible to guarantee that the best staffing level will be determined which can be associated with a certain schedule.

Moreover, we consider that patient arrival is uncertain, varying per day and hour. We address uncertainty in demand by adopting a two-stage stochastic programming formulation with fixed recourse that minimizes the expected total number of patients waiting per hour, and we note that such approach has rarely been used in the literature. In fact, although other techniques have been proposed to treat uncertainty (Ganguly et al. [2014]; Kuo [2014]; Sinreich and Jabali [2007]; Sinreich et al. [2012]), previous works have addressed different variants of the problem we consider. For instance, the models from Ganguly et al. [2014], Sinreich and Jabali [2007] and Sinreich et al. [2012] do not perform staffing and scheduling simultaneously, while Kuo [2014] uses an interactive approach for physician scheduling. We also note that our model is tested using real data from two existing EDs, unlike, e.g., Ganguly et al. [2014]. We found some related papers to this study: Daldoul et al. [2018] and EL-Rifai et al. [2015]. Both perform two-stage stochastic optimization to determine the staffing level (i.e., the scheduling problem is not addressed) for each staff category in an ED. These studies considered that patient arrival is uncertain and vary per hour of the day. Besides, both perform the staffing just considering a typical day of the week. The former considers three stages of treatment in an ED, and considers as human resources in the study both physicians and nurses; the latter extends the approach to consider beds (physical resources) and other stages of treatment in an ED (six stages of treatment). There are some differences between these studies and ours. First, we propose a generalizable and integrated model that considers uncertainty in patient arrival patterns and simultaneously accounts for ED physician staffing and scheduling problems. Second, we consider uncertainty by hour and day of the week, i.e., we consider that patient arrival rates vary not only with the hours of the day but also according to the day of the week, and we can perform the staffing and scheduling for a planning horizon that can be defined by the manager. Besides the planning horizon can be different for staffing and the scheduling. Lastly, to represent the uncertainty addressed, we generate scenarios using Sample Average Approximation (SAA) testing two scenario generation methods (which were described in Chapter 3 and which the application is detailed in Section 4.4), while the related work mentioned before just used the most common method of scenario generation.

Finally, our model may perform staffing and scheduling either in a cyclic or acyclic manner. Cyclic staffing means that the staffing level is repeated every $T$ periods across the planning horizon, while in acyclic staffing, the level always 
changes. Similarly, cyclic scheduling indicates that the physician assignment will be the same over the planning horizon, while in acyclic scheduling the allocation always changes. To our knowledge, studies in the literature only address one approach at the time, e.g., Ferrand et al. [2011] performed cyclic scheduling while Savage et al. [2015] performed acyclic scheduling. Our model is, therefore, more flexible than existing methods in the sense that it can deal with both cyclic and acyclic staffing and scheduling, and can thus be more easily adapted to different hospital realities.

From a practical perspective, we model multiple overlapped shifts, which are predefined by ED managers (but whose duration and start time may be flexible), and different physician skill categories, and we treat physicians individually considering preferences and restrictions such as shift start time and availability. We also consider differences between weekday and weekend shifts and workload. These features constitute important and realistic characteristics of ED operations. Also, our study identifies solutions to an important real problem that directly influences the quality of service, e.g., by reducing patient queue length and ultimately, the door-to-doctor time. Finally, we perform two case studies using real data from two hospitals EDs to estimate the benefits of the proposed schedules.

Table 4.1 summarizes the contributions of this chapter comparing with related works in literature. 
Table 4.1: Summary of contributions from the Chapter 4

\begin{tabular}{|c|c|c|}
\hline Chapter 4 & Literature & \\
\hline $\begin{array}{l}\text { Integrated model - simultaneously accounts for ED physi- } \\
\text { cian staffing and scheduling problems }\end{array}$ & $\begin{array}{l}\text { Neglecting the intrinsic interaction between staffing and } \\
\text { scheduling }\end{array}$ & $\begin{array}{l}\text { e.g. [Hung et al., 2007; Savage } \\
\text { et al., 2015; Vile et al., 2016] }\end{array}$ \\
\hline $\begin{array}{l}\text { Perform the staffing and scheduling for a planning horizon } \\
\text { that can be defined by the manager besides the planning } \\
\text { horizon can be different for the staffing and the scheduling }\end{array}$ & Just considering a typical day of the week & $\begin{array}{l}\text { e.g. [Daldoul et al., 2018; EL-Rifai } \\
\text { et al., 2015] }\end{array}$ \\
\hline $\begin{array}{l}\text { Stochastic Model with SAA testing two scenario genera- } \\
\text { tion methods }\end{array}$ & Stochastic Model with SAA justing using Monte Carlo & $\begin{array}{l}\text { e.g. [Daldoul et al., 2018; EL-Rifai } \\
\text { et al., 2015] }\end{array}$ \\
\hline Solution quality assessment via statistical bounds & $\begin{array}{l}\text { Do not define the number of scenarios using statistical } \\
\text { bounds }\end{array}$ & $\begin{array}{l}\text { e.g. Daldoul et al. [2018]; EL-Rifai } \\
\text { et al. [2015] }\end{array}$ \\
\hline Address physician restrictions - e.g. start time, weekend & Studies address physician preferences & $\begin{array}{l}\text { e.g. [Bard et al., 2013; Bowers } \\
\text { et al., 2016] }\end{array}$ \\
\hline Address the first assessment & Consider several stages of treatment in an ED & $\begin{array}{l}\text { e.g. Daldoul et al. [2018]; EL-Rifai } \\
\text { et al. [2015] }\end{array}$ \\
\hline Consider physicians as human resourses & Consider phisicians and nurses & $\begin{array}{l}\text { e.g. Daldoul et al. [2018]; EL-Rifai } \\
\text { et al. [2015] }\end{array}$ \\
\hline Do not consider physical resources & Consider beds as physical resources & e.g. Daldoul et al. [2018] \\
\hline
\end{tabular}


We observe from Table 4.1 and from the discussion above that we contribute with the literature in most aspects addressed in this kind of problem. The three last rows of Table 4.1 show points considered by the works in literature that are related to this study, and we do not address in this Chapter. These points will be the object of the next Chapter.

\section{2}

\section{Mathematical Model Framework}

In this section, we present the mathematical model developed to deal with the problem described above. The objective of this mathematical model is to minimize the expeted total number of patients waiting. The decisions are subject to constraints relating to contractual agreements, service requirements, and service capacity to cover fluctuating demand levels.

Table 4.2 presents the domains in which our model attributes are defined, while Tables 4.3 and 4.4 provide a complete listing of parameter and variable definitions.

Table 4.2: Sets, subsets and corresponding domains

\begin{tabular}{llll}
\hline Sets & Indexes & Domain & Description \\
\hline Shifts $(S)$ & $\mathrm{s}, \mathrm{s}^{\prime}$ & $\{1, \ldots,|\mathrm{S}|\}$ & Shifts in the ED \\
Physicians $(P)$ & $\mathrm{p}$ & $\{1, \ldots,|\mathrm{P}|\}$ & Physicians of the ED \\
Days $(D)$ & $\mathrm{d}$ & $\left\{1, \ldots, P L A N \_H O R I Z O N\right\}$ & $\begin{array}{l}\text { Days of the planning horizon } \\
\text { Hours }(H)\end{array}$ \\
Period $(T)$ & $\mathrm{h}, \mathrm{h}$, & $\{0, \ldots, 23\}$ & Hours of the day (24 hour clock format) \\
Physician Category $(K)$ & $\mathrm{t}$ & $\left\{1, \ldots, P L A N \_P E R I O D\right\}$ & $\begin{array}{l}\text { Period over which staffing level is repeated } \\
\text { Scenarios }(S C)\end{array}$ \\
Physician in certain cate- & $\mathrm{c}$ & $\{1, \ldots,|\mathrm{K}|\}$ & Physician skill categories \\
gory $\left(P_{k}\right)$ & Subset of Physicians $(P)$ & Possible realization scenarios \\
Weekdays $(W D)$ & - & Subset of Days $(D)$ & \\
Weekends $(W)$ & - & Subset of Days $(D)$ & Weekdays on the planning horizon \\
Night_Shifts $(N I G H T)$ & - & Subset of Shifts $(S)$ & Weekend days on the planning horizon \\
N_Shifts $(N S)$ & - & Subset of Shifts $(S)$ & Night shifts \\
Y_Shifts $(Y S)$ & - & Subset of Shifts $(S)$ & Shifts that do not allow combined allocation \\
Physicians_Week $(P 1)$ & - & Subset of Physicians $(P)$ & Shifts that allow combined allocation \\
Physicians_Both $(P 2)$ & - & Subset of Physicians $(P)$ & Physicians that work only week days \\
\hline
\end{tabular}


Table 4.3: Model parameters

\begin{tabular}{|c|c|c|}
\hline Parameters & Description & Unit \\
\hline $\mathrm{PROB}_{c}$ & Probability of each scenario $c$ & - \\
\hline PLAN_HORIZON & Number of days in the planning horizon & - \\
\hline PLAN_PERIOD & Period (number of days) over which the staffing level is repeated & - \\
\hline$D U R_{s}$ & Duration of each shift $s$ & Hour \\
\hline$W L \_W E E K_{p}$ & Number of hours available for each physician $p$ in the week & Hour \\
\hline$W L \_W E E K E N D_{p}$ & Number of hours available for each physician $p$ in the weekend & Hour \\
\hline$W L \_T O T A L_{p}$ & Total Number of hours available for each physician $p$ & Hour \\
\hline$M A X \_A S S I G N 1$ & Maximum number of assignments per day & - \\
\hline$R E L \_S H I F T S_{s s^{\prime}}$ & Indication if shift $s$ can be combined with shift $s^{\prime}$ & $\{0,1\}$ \\
\hline$M A X \_A S S I G N 2$ & Maximum number of assignments per day & - \\
\hline$M A X \_T Y P E_{p s}$ & Maximum number of assignments in a certain shift type & Hour \\
\hline$R E L \_P H Y S H I_{p s}$ & Indication if physician $p$ can be schedule for shift $s$ & $\{0,1\}$ \\
\hline$S T A R T \_C O M B_{s h p}$ & Indication of start time $h$ of each shift $s$ for each physician $p$ & $\{0,1\}$ \\
\hline$M I N_{k h}$ & Minimum number of physicians required in hour $h$ for each skill category $k$ & - \\
\hline$C A P \_M I N_{h t}$ & Minimum number of physicians required per hour $h$ and day $t$ & - \\
\hline$D A Y \_P E R I O D_{d t}$ & Indication if day $d$ is included in period $t$ & 0,1 \\
\hline$D E M_{h t}^{c}$ & Demand (arrival of patients for each period $t$, hour $h$ and scenario $c$ ) & - \\
\hline$C A P$ & Capacity (service rate per physician) & - \\
\hline$I \_P H Y S I C I A N_{s d p}$ & Indication if physician $p$ is available on day $d$ and shift $s$ & $\{0,1\}$ \\
\hline
\end{tabular}

Of note, parameters that indicate whether shift combinations are allowed $\left(R E L \_S H I F T S_{s s^{\prime}}\right)$ and whether any given physician is available to cover certain shifts $\left(I_{-}\right.$PHYSICIAN $\left.N_{s d p}\right)$ are generated as part of a pre-processing stage. In the case of shift rules, information regarding which shifts allow combined allocation is used to construct a binary matrix where the pairs of shifts that allow combined allocation receive value 1 and the others receive 0 . Combined allocation is the allocation of the same physician for 2 or more different shifts on the same day and shifts. This case can happen if the shifts are non-overlapped, sequential, and respect the maximum number of consecutive hours of work. In the case of physicians' availability, a binary matrix is constructed with information on which physician is available to be assigned on each day and shift so that each entry takes value 1 when the physician is available and 0 otherwise.

The parameter PLAN_HORIZON is a multiple of the PLAN_PERIOD. With these two parameters, we define if the staffing and scheduling are cyclic or acyclic. Figure 4.2 shows two examples to explain how to use these parameters to do staffing cyclic or acyclic. 


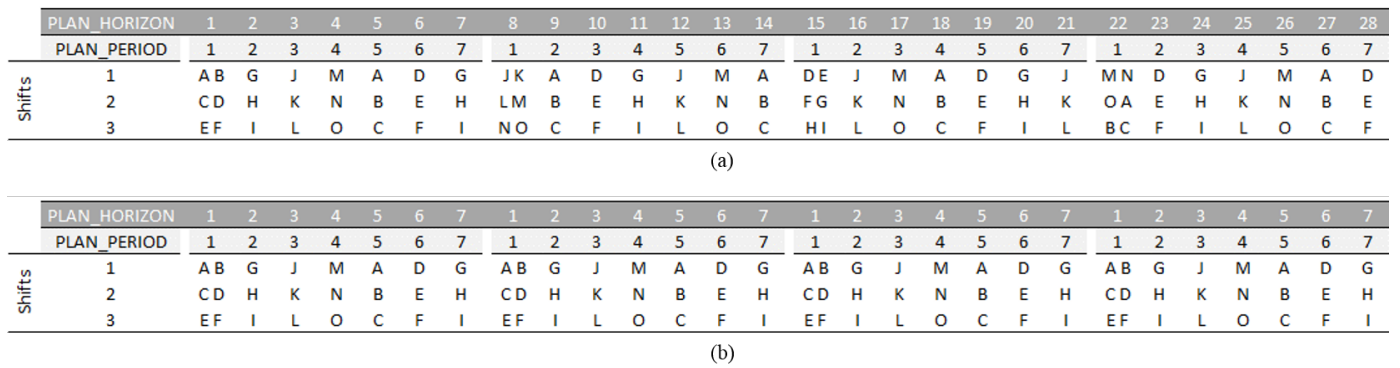

Figure 4.2: Example of PLAN_HORIZON and PLAN_PERIOD behavior

Figure 4.2 (a) shows staffing and scheduling for 4 -weeks given that the staffing is cyclic, and the scheduling is acyclic. Hence, in the first day of the PLAN_HORIZON, two physicians are assigned for the shift 1 , and we also have two physicians assigned for days 8, 15 and 22, because these days corresponds to day 1 of PLAN_PERIOD. It means that the decisions regarding staffing will be made for one week and repeated (cyclic) four times over the PLAN_HORIZON while the decisions related to scheduling are not repeated (acyclic) and will be made for each day. Thus, we can observe that in the first day of the PLAN_HORIZON the physicians $\mathrm{A}$ and $\mathrm{B}$ are allocated, in day 8 the physicians $\mathrm{J}$ and $\mathrm{K}$ are allocated, and so on, i.e., different physicians. Figure 4.2 (b) shows staffing and scheduling for 4-weeks, given that the staffing and the scheduling are cyclic. It means that the decisions regarding staffing and scheduling will be made for one week and repeated (cyclic) four times.

The parameter $D A Y \_P E R I O D_{d t}$ makes the correspondence between the days of the PLAN_HORIZON and PLAN_PERIOD. Looking for Figure 4.2 (a) we can see that, for example, days $1,8,15$ and 22 of the PLAN_HORIZON corresponds to day 1 of the PLAN_PERIOD. Thus, $D A Y \_P E R I O D_{1,1}=1, D A Y \_P E R I O D_{8,1}=1, D A Y \_P E R I O D_{15,1}=1$, $D A Y \_P E R I O D_{22,1}=1$, and the others assume value 0 .

Table 4.4: Model variables

\begin{tabular}{lll}
\hline Variables & Description & Domain \\
\hline wait $_{h t}^{c}$ & Number of patients waiting in each hour $h$, each period $t$ and each scenario $c$ & $\mathbb{R}_{+}$ \\
$x_{s d p}$ & Decision if physician $p$ is assigned in the shift $s$ in the day $d$ & $\{0,1\}$ \\
$i c_{h t}^{c}$ & Idle capacity in each hour $h$, period $t$ and each scenario $c$ & $\mathbb{R}_{+}$ \\
$n_{h t}$ & Number of physicians required for each hour $h$ and each period $t$ & $\mathbb{Z}_{+}$ \\
\hline
\end{tabular}

Constraints represent both necessary and desirable scheduling conditions. The former includes service requirements and contractual agreements, which 
were modeled as hard constraints. The latter is associated with demand coverage, being modeled as soft constraints. Recall that we account for uncertainty in demand, but physician assignment is the first-stage variable. Our objective is thus to minimize the expected total number of patients waiting.

Our model can be easily adapted to reflect the operational characteristics of several EDs. To provide greater clarity in the problem's notation, the domains of summations are omitted except when the summation is evaluated only on a subset of the natural domain. When there is no mention of this fact, its domain should be considered as the original set to which the index refers. The proposed mathematical formulation is as follows:

$$
\operatorname{MinZ}=\sum_{h} \sum_{t} \sum_{c} P R O B_{c} w_{a i t}^{c}
$$

The objective function in (4-1) consists of minimizing the expected value of the total number of patients waiting.

Subject to:

$$
\begin{gathered}
\sum_{d \in W D} \sum_{s} x_{s d p} D U R_{s} \leq W L \_W E E K_{p} \quad \forall p \in P 1 \\
\sum_{d \in W} \sum_{s} x_{s d p} D U R_{s} \leq W L \_W E E K E N D_{p} \quad \forall p \in P 2 \\
\sum_{d} \sum_{s} x_{s d p} D U R_{s} \leq W L \_T O T A L_{p} \quad \forall p \in P
\end{gathered}
$$

Constraints (4-2)-(4-4) ensure that the maximum allocation time for each physician in the planning horizon is respected. Constraint (4-2) ensures that the sum of the shift durations to which the physician has been assigned in the week is less than or equal to the total number of hours available to the week. This applies for physicians that work only on weekdays. Constrains (4-3) enforces that the sum of the shift durations to which the physician has been assigned on weekends is less than or equal to the total number of hours available to the weekend. Constraint (4-4) enforces that the total number of hours assigned in the entire planning horizon is less than or equal to the total number of hours available.

$$
\sum_{s} x_{s d p} \leq M A X \_A S S I G N 1 \quad \forall p \in P, \forall d \in D
$$

Constraint (4-5) guarantees that each physician is allocated to a maximum number of shifts per day.

$$
\begin{array}{r}
x_{s d p}+x_{s^{\prime} d p}\left(1-R E L \_S H I F T S_{s s^{\prime}}\right) \leq M A X \_A S S I G N 1 \\
\forall p \in P, \forall d \in D, \forall s \in S, \forall s^{\prime} \in Y S \mid s \neq s^{\prime} \wedge \neg R E L \_S H I F T S_{s s^{\prime}}
\end{array}
$$


Constraint (4-6) ensure that, for any given day, physicians are not assigned to any of the following: shifts that do not respect the minimum interval between assignments; shifts that do not respect the maximum allowed consecutive hours of work; overlapped shifts.

$$
\begin{array}{r}
x_{s d p}+\sum_{s^{\prime} \mid s^{\prime} \neq s} x_{s^{\prime} d p} R E L \_S H I F T S_{s s^{\prime}} \leq M A X \_A S S I G N 2 \\
\forall p \in P, \forall d \text { in } D, \forall s \in Y S \wedge \exists s^{\prime} \mid R E L \_S H I F T S_{s s^{\prime}}
\end{array}
$$

Constraint (4-7) allows physicians to be assigned to different shifts on the same day, as long as the shifts are non-overlapped and sequential and the physician belongs to the group of physicians who can work on more than one shift per day.

$$
\sum_{s \in N i g h t} x_{s d p}+x_{s^{\prime}, d+1, p} \leq M A X \_A S S I G N 1 \quad \forall p \in P, \forall d \in D, \forall s^{\prime} \in S
$$

Constraint (4-8) enforces that physicians allocated on the night shift will not be allocated to any other shift on the same day and can only be allocated on the night shift of the following day, thus guaranteeing that the minimum interval between assignments is respected in the event of physicians being allocated on night shifts.

$$
\sum_{d} x_{s d p} \leq M A X \_T Y P E_{p s} \quad \forall p \in P, \forall s \in S \mid R E L \_P H Y S H I_{p s}
$$

Constraint (4-9) guarantees that during the planning horizon each physician is allocated at most the number of times allowed for each type of shift.

$$
\begin{array}{r}
\sum_{p i n P_{k}} \sum_{d} \sum_{h^{\prime}} x_{s d p} S T A R T_{\_} C O M B_{s h^{\prime} p} \geq M I N_{k h} \\
\forall h \in H, \forall d \in D, \forall k \in K
\end{array}
$$

Constraint (4-10) ensures that the minimum number of physicians per hour required for each physician category will be met.

$$
\begin{array}{r}
\sum_{p} \sum_{d} \sum_{h^{\prime}} x_{s d p} S T A R T \_C O M B_{s h^{\prime} p} \geq n_{h t}+C A P \_M I N_{h t} \\
\forall h \in H, \forall d \in D, \forall t \in T \mid D A Y \_P E R I O D d t
\end{array}
$$

Constraint (4-11) enforces that the schedule meets hourly staffing levels. 
Here, with parameter $C A P \_M I N_{h t}$, we guarantee, although we are doing the staffing for first assessment, that there are physician enough to be allocated every day and time to attend the other areas of the ED. Queue and idle capacity are computed through (4-12)-(4-13) based on the number of physicians required per hour to meet demand.

$$
w_{a i t}^{c}+i c_{h t}^{c}=D E M_{h t}^{c}-n_{h t} C A P \quad \forall h \mid h=0, \forall t \in T, \forall c \in S C
$$

$$
w_{a i t}^{c}+i c_{h t}^{c}=D E M_{h t}^{c}+w a i t_{h-1, t}^{c}-n_{h t} C A P \quad \forall h \mid h \geq 1, \forall t \in T, \forall c \in S C
$$

Finally, constraints (4-14)-(4-17) define the domain of the decision variables.

$$
\begin{array}{r}
\text { wait }_{h t}^{c} \in \mathbb{R}_{+}, \quad \forall h \in H, \forall t \in T, \forall c \in S C \\
i c_{h t}^{c} \in \mathbb{R}_{+}, \quad \forall h \in H, \forall t \in T, \forall c \in S C \\
x_{s h p} \in\{0,1\}, \quad \forall s \in S, \forall h \in H, \forall p \in P \mid \exists I_{-} \text {PHYSICIAN } N_{s d p} \\
n_{h t} \in \mathbb{Z}_{+}, \quad \forall h \in H, \forall t \in T
\end{array}
$$

\section{3}

\section{Simulation Modeling Framework}

Here the simulation follows what is described in Section 3.2.4. The simulation model developed is based in Figure 4.1. The input to our simulation model is the schedule resulting from the optimization model (i.e., the number of physicians assigned to each day and hour). The assumption made in the optimization model that he system is initially empty is relaxed in the simulation model.

\section{4}

\section{Numerical Experiments}

For numerical experiments, we present the application of the model shown in Section 4.2 to two real case studies on the Brazilian hospitals. The scenario generation methods were implemented in MATLAB R2013a. The mathematical programming model was implemented and solved using AIMMS 4.26 and GUROBI 7.5 with default settings. The solutions were achieved on an Intel i7 3.3 GHz 64GB RAM computer. The simulation model was implemented 
using Arena Simulation software version 14.7. All statistical analyses were performed using $\mathrm{R}$ software version 3.3.1.

The computational experiments using SAA were performed considering the two scenario generation methods - Monte Carlo Sampling (MCS) and Latin Hypercube Sampling (LHS) - presented in Chapter 3.1. Following the Step 1 of the solution framework proposed in Section 3.2, by performing a fit test in the data we conclude that the number of patients arriving every hour and day follows a Poisson distribution for the two case studies (we will show the results of the fit test later). Hence, we used this probability distribution to perform the random sample draws in the generation of scenarios regarding the arrival of patients (Demand). For the Step 2 of the solution framework proposed in Section 3.2 we define the parameters used in the SAA procedure defined in the Algorithm 1. For lower bound calculation, our in-sample analysis, we use 50 replications $(M=50)$ and we start using 100 scenarios $(N=100)$. We use an additional independent set of samples of 10,000 independent scenarios ( $T=1$ and $\bar{N}=10,000)$, representing the true distribution, to calculate the upper bound, our out-of-sample analysis. If the stop criterion presented in the Section 3.2.2 is not achieved the required number of scenarios $N$ is increased by 100 .

\section{5 \\ Case study in the Hospital A}

Patient arrival data in the ED's Clinical Medicine was made available by the hospital between January 2015 and April 2016. Thus we work with 11,664 hours of ED data representing about 85,000 patients. The ED performs an average of 5,000 medical encounters per month for Clinical Medicine (CM), which is attended to by general practitioners and/or cardiologists. Some physicians act as both general practitioners and cardiologists, while others work as either general practitioners or cardiologists. Next, we detail such characteristic and how they were used to calibrate model parameters.

For each planning period, CM is staffed by 44 physicians, bein the total number of work hours available equal to 3,888 hours. On any given day, physicians are assigned to one or several of seven overlapped shifts, which are shown in Figure 4.3. The duration of each shift $\left(D U R_{s}\right)$ is typically 6 hours, and the night shift is 12 hours long. A physician may be allocated to more than one shift per day as long as the shifts are non-overlapped and sequential, and their combined length (in hours) does not surpass the maximum number of allowable consecutive work hours (12h). Additional scheduling rules and contractual agreements include: (i) the sum of the shift lengths to which a 
physician is assigned must respect the maximum number of hours available for each physician in a four-week cycle (planning horizon), both in terms of total number of hours available and number of hours available in the weekend. Total number of hours available $\left(W L_{-} T O T A L_{p}\right)$ varies for each physician, ranging from 48 to 144 hours, and number of hours available in the weekend $\left(W L \_W E E K E N D_{p}\right)$ also varies for each physician, being 12, 24 , or 36 hours. Hence, parameter PLAN_HORIZON takes the value of 28 days and parameter PLAN_PERIOD takes the value of 7 days. Of note, the Friday night shift is accounted for within the number of hours available in the weekend; (ii) a minimum of four physicians, including at least one cardiologist, must always be available in the ED, meaning that parameter $C A P \_M I N_{h t}$ takes the value of 4 and $M I N_{k}$ takes value 1; (iii) a minimum time interval of six hours between consecutive assignments must be met, except when a physician is assigned to the night shift, in which case the interval must be of at least 12 hours; (iv) each physician may only be assigned to a maximum of 12 consecutive work hours; (v) overlapped shifts may not be used for combined allocation; (vi) non-overlapped and sequential shifts allow combined allocation; (vii) staffing levels must be repeated weekly. This last constraint, imposed by ED managers, forces the same number of physicians to be repeatedly allocated on each day across the four weeks of a planning horizon, thus ensuring the same level of service throughout the planning horizon. For instance, if five physicians were allocated to shift 1 (see Figure 4.3) on Monday of week 1, this same number must be allocated to the first shift on Mondays of weeks 2, 3 and 4.

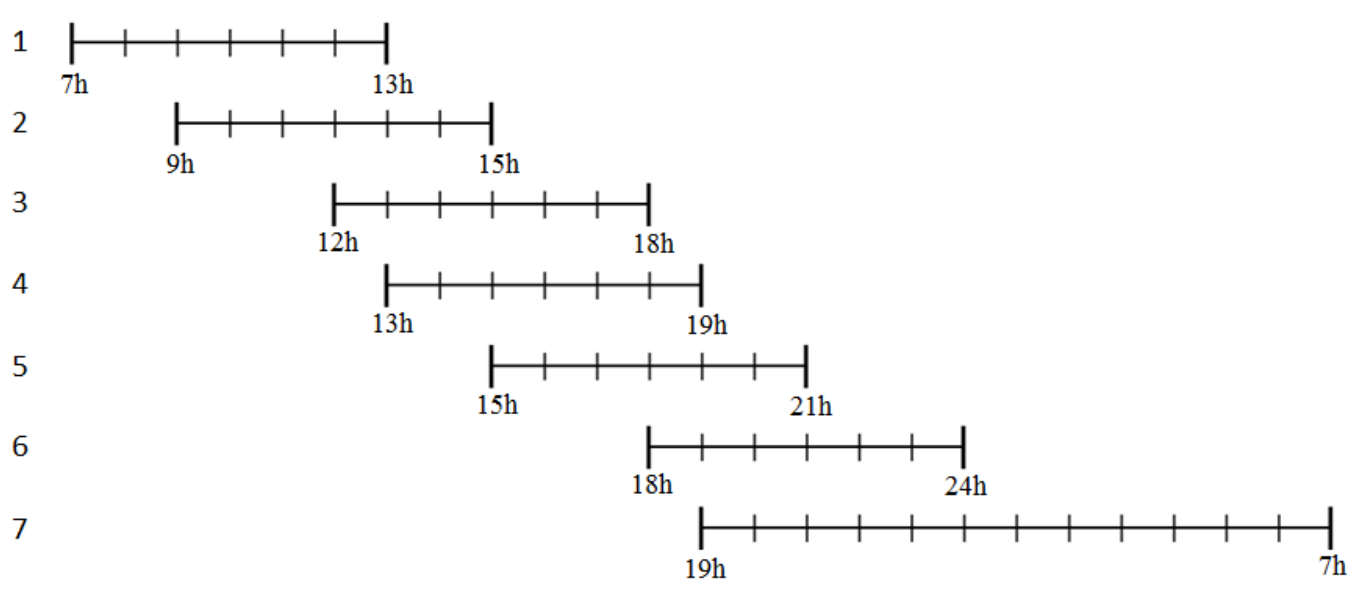

Figure 4.3: Shift distribution adopted by Clinical Medicine in the ED of Hospital A (24 hour clock format) 
Here, the staffing and scheduling for 4 -weeks given that the staffing is cyclic over the weeks, i.e., the staffing level are repeated week by week, and the scheduling is no cyclic. We set the PLAN_HORIZON $=28$ and the PLAN_PERIOD $=7$.

\subsection{1}

\section{Physician staff}

As previously mentioned, in the staff scheduling literature, the staffing phase involves determining the number of personnel, with a certain set of skills, required to meet service demand within a given time frame (note that in other contexts, this step is known as sizing). Presently, staffing at the real ED we consider here is performed through an empirical analysis of demand behavior. Generating a manually-defined schedule (shown in Table D.1 of Appendix D) is notably quite a laborious task. More importantly, its solution is not necessarily optimal, nor does it guarantee that all scheduling rules and contractual agreements are met. Another aspect of the current schedule's inefficiency is reflected in the current levels of demand and capacity at the ED. Figure 4.4 shows average waiting time per hour of the day considering the current schedule in the ED of Hospital A. These are data from the simulation model using the current schedule adopted by this ED. It is clear from Figure 4.4 that the waiting time increase along of the day. This happens beacause there are several points in time when demand greatly exceeds capacity; this occurs because the current schedule does not account for uncertainty in patient arrival.

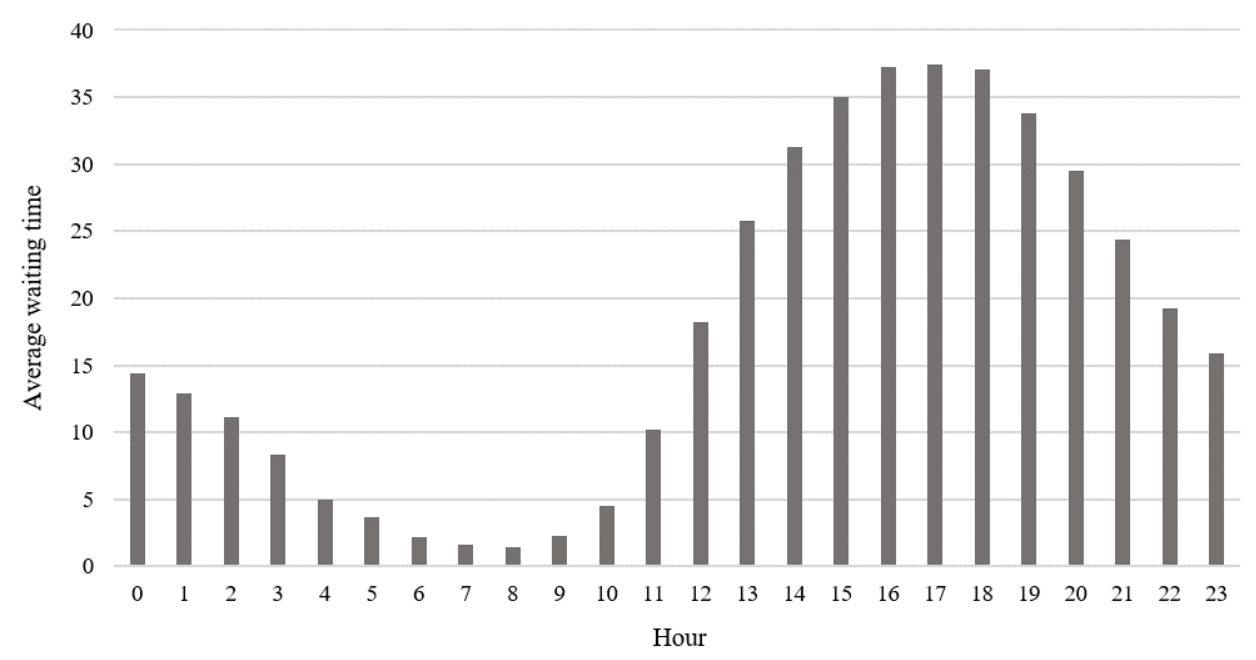

Figure 4.4: Average waiting time per hour in Hospital A 


\subsection{2}

\section{Physician scheduling}

The number of patients in the queue is an important measure of performance for the hospital we analyze. The ED managers' main concerns relates to the occurrence and length of queues, which directly affect a patient's wait time and their total length of stay in the ED. In this context, ED managers are particularly concerned about controlling queue length to avoid the need to reallocate resources unexpectedly.

At this point, it is worth mentioning that the current manually-defined schedule (see Table D.1 on the Appendix D) not only fails to address the concerns of ED managers regarding the occurrence and length of patient queues but also does not respect several of the scheduling mentioned above rules. For one, the requirement that at least four physicians should be available every hour is not consistently met. Moreover, the current roster does not guarantee that a cardiologist will always be available in CM. Additionally, in some cases, the total or weekend maximum workload is not respected, and in one instance, the minimum interval between assignments is not met. Finally, the current schedule does not ensure that staffing levels for each scheduling period are repeated weekly. In total, our analysis revealed 420 hours of scheduling rule violations of a total os 4,416 hours analyzed related to a schedule for a planning horizon of 4 -weeks. In this context, the ultimate goal of the physician scheduling phase is to ensure that the available physician workforce is assigned in such a way to minimize patient queues while taking into account all schedule requirements and contractual agreements.

\subsection{3}

\section{Simulation model}

For the simulation model of the ED of Hospital A in addition to the simulation model assumptions cited in section 3.2.4, we consider an estimated service time of 20 minutes, as explained in Section 4.5.1, as an average for the exponential distribution used in the simulation model. We assume that the patients arrival follows a Poisson distibutions as showed in the fit test performed using the real data (Figure D.1 in the Appendix D). In terms to define whether the pattern ot patients arrival is different over the days of week and the hour of the day, we conduct a Kruskal-Wallis test which results indicated a statistically significant difference between patient interarrival times for each day of the week and hour (Kruskal-Wallis test, chi-squared $=30263$, $\mathrm{df}=23$, $\mathrm{p}$-value $<2.2 \mathrm{e}-16)$. We note that the Kruskal-Wallis test was used due to the non-normality of the data set (Shapiro test, $\mathrm{W}=0.7112$, p-value 
$<2.2 \mathrm{e}-16)$. To determine which groups were different, and hence define which hours and days of the week could be considered to have the same arrival rate, we then performed a multiple comparison test (results shown in Table D.2 of the Appendix D). These tests is performed using the data of patients arrival in the ED for the period analyzed, as mentioned before we are working with about 85,000 patients.

The current manually-defined ED schedule was used to define the warmup period and number of required replications, and to validate our simulation model. A warm-up period of 15-hours and a total of 110 replications were found to ensure that system occupancy corresponded to real starting conditions. Each replication simulated 11,664 hours of operations, which amounts to the total length of the period for which ED data was made available. Model validation was performed by comparing the real number of patients treated in the ED $(85,619$ patients) with the average number of patients serviced in the simulation model (85,572 patients), which yielded a variation of approximately $0.05 \%$ in the total number of treated patients. We considered such variation to be negligible and hence indicative of the simulated model's accuracy

\subsection{4}

\section{Numerical results of the case study in the Hospital A}

We start defining the number of scenarios that should be used in the experiments. For this, we run preliminary tests as specified in Section 3.2.2 using Monte Carlo Sampling (MCS) and Latin Hypercube Sampling (LHS). The size of the models (i.e.,variables and constraints), average execution times, and their variances are shown in Table E.1 of Appendix E. The average solution time of the experiments was $15.27 \mathrm{CPU}$ seconds for 100 scenarios and 69.26 CPU seconds for 1,000 scenarios.

Table 4.5 present the results for each experiment in terms of the objective function for the in-sample and out-of-sample tests and the optimality gap showing the average and standard deviation. 
Table 4.5: Experiment results: in-sample and out-of-sample analysis and estimative of the optimality gap

\begin{tabular}{|c|c|c|c|c|c|c|c|}
\hline & & \multicolumn{3}{|c|}{ Monte Carlo Sampling } & \multicolumn{3}{|c|}{ Latin Hypercube Sampling } \\
\hline & & $\begin{array}{l}\text { Objective } \\
\text { Function } \\
\text { In Sample }\end{array}$ & $\begin{array}{l}\text { Objective } \\
\text { Function Out } \\
\text { of Sample }\end{array}$ & Gap & $\begin{array}{l}\text { Objective } \\
\text { Function } \\
\text { In Sample }\end{array}$ & $\begin{array}{l}\text { Objective } \\
\text { Function Out } \\
\text { of Sample }\end{array}$ & Gap \\
\hline \multirow{2}{*}{100 Scenarios } & Average & 0.138 & 0.481 & 248.629 & 0.205 & 0.386 & 87.944 \\
\hline & St.Dev & 0.068 & 0.069 & 49.769 & 0.059 & 0.036 & 3.616 \\
\hline \multirow{2}{*}{200 Scenarios } & Average & 0.194 & 0.374 & 92.834 & 0.274 & 0.325 & 18.525 \\
\hline & St.Dev & 0.043 & 0.027 & 13.768 & 0.034 & 0.019 & 6.972 \\
\hline \multirow{2}{*}{300 Scenarios } & Average & 0.225 & 0.354 & 57.217 & 0.274 & 0.306 & 11.343 \\
\hline & St.Dev & 0.042 & 0.029 & 13.038 & 0.028 & 0.014 & 5.005 \\
\hline \multirow[b]{2}{*}{400 Scenarios } & Average & 0.241 & 0.339 & 40.927 & 0.281 & 0.296 & 5.407 \\
\hline & St.Dev & 0.039 & 0.022 & 9.185 & 0.027 & 0.009 & 3.136 \\
\hline \multirow[b]{2}{*}{500 Scenarios } & Average & 0.240 & 0.330 & 37.617 & 0.282 & 0.294 & 4.289 \\
\hline & St.Dev & 0.027 & 0.016 & 6.689 & 0.022 & 0.008 & 2.893 \\
\hline \multirow[b]{2}{*}{600 Scenarios } & Average & 0.248 & 0.320 & 29.156 & 0.290 & 0.290 & 0.012 \\
\hline & St.Dev & 0.028 & 0.019 & 7.812 & 0.019 & 0.007 & 2.287 \\
\hline \multirow{2}{*}{700 Scenarios } & Average & 0.255 & 0.318 & 24.553 & 0.288 & 0.288 & -0.031 \\
\hline & St.Dev & 0.026 & 0.018 & 6.926 & 0.013 & 0.004 & 1.405 \\
\hline \multirow{2}{*}{800 Scenarios } & Average & 0.263 & 0.312 & 18.760 & 0.289 & 0.286 & -0.957 \\
\hline & St.Dev & 0.026 & 0.017 & 6.425 & 0.014 & 0.004 & 1.345 \\
\hline \multirow{2}{*}{900 Scenarios } & Average & 0.269 & 0.313 & 16.698 & 0.285 & 0.286 & 0.168 \\
\hline & St.Dev & 0.029 & 0.016 & 5.814 & 0.012 & 0.003 & 0.942 \\
\hline \multirow{2}{*}{1000 Scenarios } & Average & 0.264 & 0.309 & 17.041 & 0.288 & 0.286 & -0.870 \\
\hline & St.Dev & 0.027 & 0.015 & 5.835 & 0.012 & 0.003 & 1.010 \\
\hline
\end{tabular}

The results suggest that the variability is regarding the number of scenarios considered to obtain the solution and tend to reduce as we consider more scenarios. This effect is related with the fact that, in general, a larger number of scenarios leads to a more comprehensive staffing and scheduling profile in terms of its ability to handle higher demands, which makes the system more robust concerning variations in the demand and consequently achieves small fluctuations in second-stage costs. Regarding the optimality gap we observe that using the LHS method we have a faster convergence to a gap $\leq 1 \%$ while using the MCS method even with 1,000 scenarios the gap still large, $\geq 17 \%$. We notice that optimality gap achieve the stop criterion defined for about 600 scenarios using the LHS, being the gap $0.012 \%$ and the $95 \%$ level of confidence interval (CI) is 0.012, thus the gap considering the $\mathrm{CI}$ is lower than 1\%. We show the CI for each experiment in Table F.1 of Appendix F. So all results presented hereafter will be using this scenario setting.

We evaluate the average number of solutions in the in-sample analysis (showed in Tables F.2 and F.3 of Appendix F) and we also evaluate the average number of dominant solutions in the in-sample analysis (showed in Tables F.4 - F.23 of Appendix F.3). Even the analysis of dominant solutions for the MCS 
method point that using 1,000 scenarios the average number of solution is 1.0, the optimality gap analysis does not allow us to affirm that this solution is the true solution of the problem. However, by doing the same analysis using the LHS method we have the hypothesis that we achieve solutions that are close to the real optimal solution of the problem, i.e. the solutions have low gap, within the established criterion, and the number of dominant solutions tends to 1 , so we can consider of the true solution for the problem according to the SAA technique explain in the Chapter 3.

At this point, it is worth pointing out that we perform a preliminary study in the Hospital A using just historical data (HD) as scenarios in the optimization model. This preliminary study is presented in Appendix G. Using the historical data we could generate only 68 demand scenarios, each of which corresponded to the realization of real ED historical demand.

Table 4.6 contrasts indicators of service quality for the current schedule with those obtained through discrete-event simulation of the optimal schedule generated by model using SAA and using historical data. For results related to the models, in addition to the value of each indicator, we present the percentage variation for: model using historical data vs current schedule $\left(\Delta_{1} \%\right)$; model using SAA vs current schedule $\left(\Delta_{2} \%\right)$; model using SAA vs model using historical data $\left(\Delta_{3} \%\right)$. In addition to the value of each indicator, we also report the $95 \%$ level of confidence interval (CI).

Several points are worthy of mention: first, note the reduction of approximately $73 \%$ in the frequency of queues, which is greater reduction than the one shown when using historical data. Moreover, the frequency of queue with more than 10 patients (one of the main quality indicators defined by managers from the real ED we are analyzing) decrease approximately $96 \%$, which is considerably lower than both the current schedule and the schedule generated using historical data. Additionally, with the model using SAA, we achieve a reduction of approximately $92 \%$ in the number of patients in the queue, while the average time door-to-doctor decrease by about $92 \%$ concerning the current schedule.

Table 4.6: Discrete-event simulation results for case study in the Hospital A using SAA

\begin{tabular}{|c|c|c|c|c|c|c|c|c|c|}
\hline & \multirow{2}{*}{\multicolumn{2}{|c|}{ Current Allocation }} & \multirow{2}{*}{\multicolumn{3}{|c|}{ Model using HD }} & & & & \\
\hline & & & & & & \multicolumn{4}{|c|}{ Model using SAA } \\
\hline & Value & CI & Value & CI & $\Delta_{1} \%$ & Value & $\mathrm{CI}$ & $\Delta_{2} \%$ & $\Delta_{3} \%$ \\
\hline Number of patients & 85623.66 & 78.28 & 85613.22 & 70.94 & $-0.01 \%$ & 85622.92 & 70.96 & $0.00 \%$ & $0.01 \%$ \\
\hline Frequency of queue (\%) & 24.05 & 0.00 & 9.00 & 0.00 & $-62.58 \%$ & 6.45 & 0.00 & $-73.18 \%$ & $-10.60 \%$ \\
\hline Frequency of queue $>10$ patients $(\%)$ & 9.84 & 0.00 & 0.91 & 0.00 & $-90.75 \%$ & 0.35 & 0.00 & $-96.44 \%$ & $-5.69 \%$ \\
\hline Frequency of queue $<=10$ patients (\%) & 14.21 & 0.00 & 8.08 & 0.00 & $-43.14 \%$ & 6.10 & 0.00 & $-57.07 \%$ & $-13.93 \%$ \\
\hline Average number in queue & 3.00 & 0.08 & 0.42 & 0.01 & $-86.00 \%$ & 0.23 & 0.01 & $-92.33 \%$ & $-6.33 \%$ \\
\hline Average door-to-doctor time (min) & 24.53 & 0.63 & 3.41 & 0.10 & $-86.10 \%$ & 1.89 & 0.06 & $-92.30 \%$ & $-6.20 \%$ \\
\hline Total numer of physicians hours used & 3522.00 & - & 3888.00 & - & $10.39 \%$ & 3888.00 & - & $10.39 \%$ & $0.00 \%$ \\
\hline
\end{tabular}


Figure 4.5 shows the comparison of the current empirically-defined schedule of ED in the Hospital A and the schedule from the proposed model in terms of service demand and capacity. From the Figure, we observed that with the schedule from the proposed model, we were able to better adapt the capacity to the demand of the day achieving better waiting times.

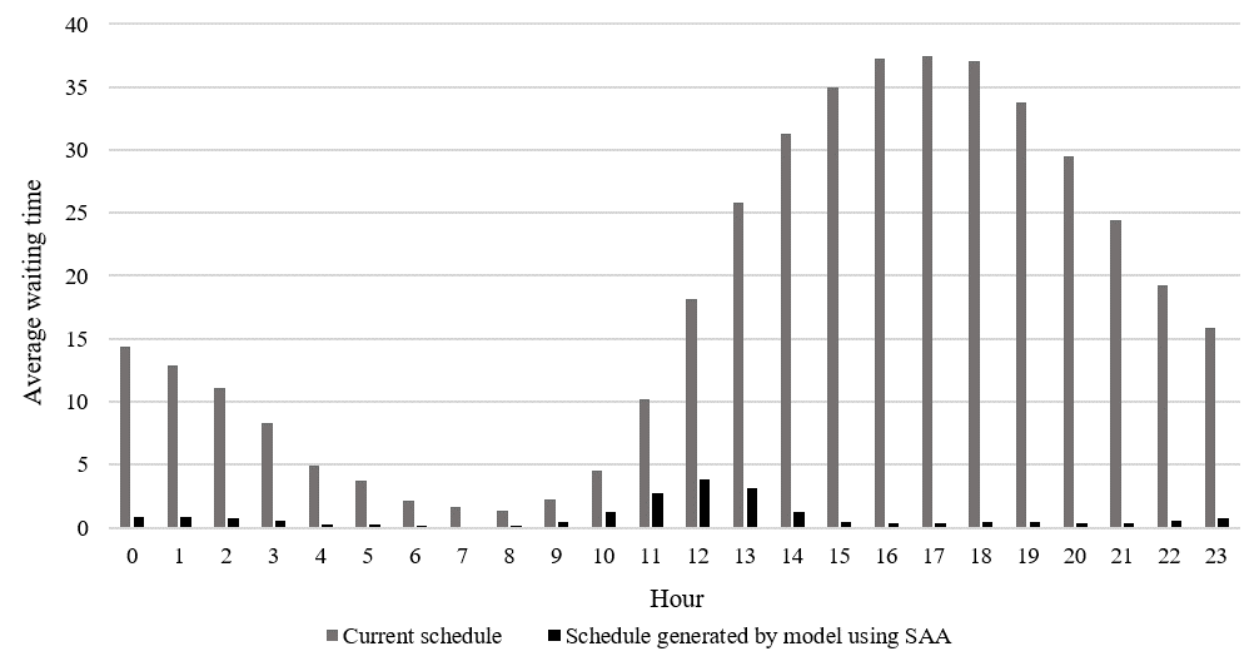

Figure 4.5: Average waiting time in Hospital A comparing the current empirically-defined schedule of ED in the Hospital A and the schedule from the proposed model

Figure 4.6 presents the histograms of idle capacity and queue frequency for the current schedule and the schedule generated by Model using SAA. As shown in Figure 4.6, we observe that queue using the scenarios generation are even smaller than that presented in G.1(b) and also less frequent. 


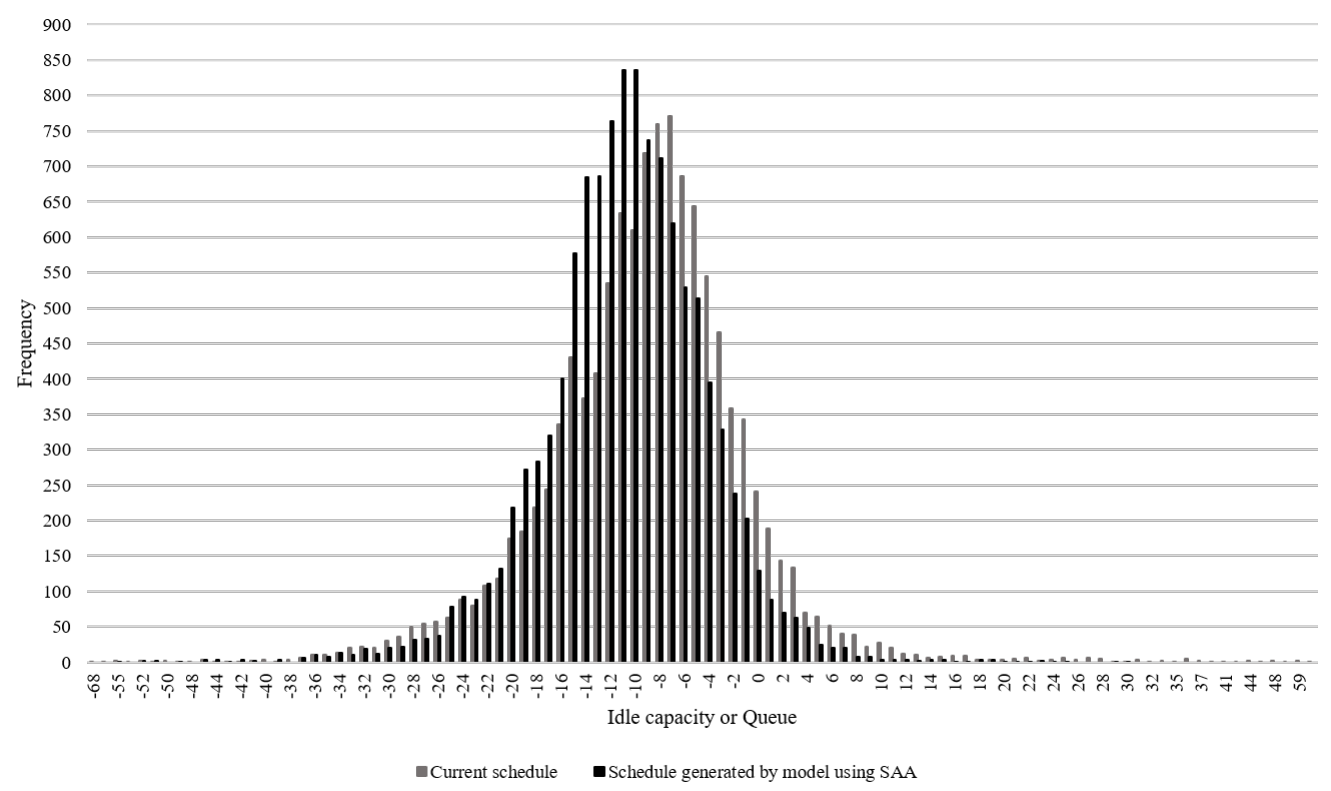

Figure 4.6: Histogram of frequency distribution of idle capacity (negative values) or queue (positive values) for the current schedule and the schedule generated by model using SAA

Taken together, these results indicate that the schedule generated by model using SAA takes advantage of the wide representation of the uncertainty in the arrival of patients by the generation of scenarios and thus achieves better results than those obtained using only historical data.

\section{6}

\section{Case study in the Hospital B}

Patient arrival data in the ED of Hospital B was available for the period of October 2017 - July 2018. Thus we work with 6,585 hours of ED data representing about 73,000 patients. The ED performs an average of 5,200 medical encounters per month for Clinical Medicine (CM), which is attended to by general practitioners and/or cardiologists. Similarly to the case study I, some physicians act as both general practitioners and cardiologists, while others work as either general practitioners or cardiologists. Here, of the total number of patients in the Clinical medicine, we consider only those patients who go straight from triage to first care, discounting patients who need vital care and patients who go direct to the beds.

For each planning period, CM is staffed by 85 physicians, bein the total number of work hours available equal to 1,457 hours. Moreover, on any given day, physicians are assigned to one or several of eleven overlapped shifts, which are shown in Figure 4.7. The shifts differ in type according to the duration 
$\left(D U R_{s}\right)$ of each one, so the shifts can be of type $6 \mathrm{~h}, 9 \mathrm{~h}, 10 \mathrm{~h}$, and $12 \mathrm{~h}$. Shifts of type $10 \mathrm{~h}$ are used only on the weekends.

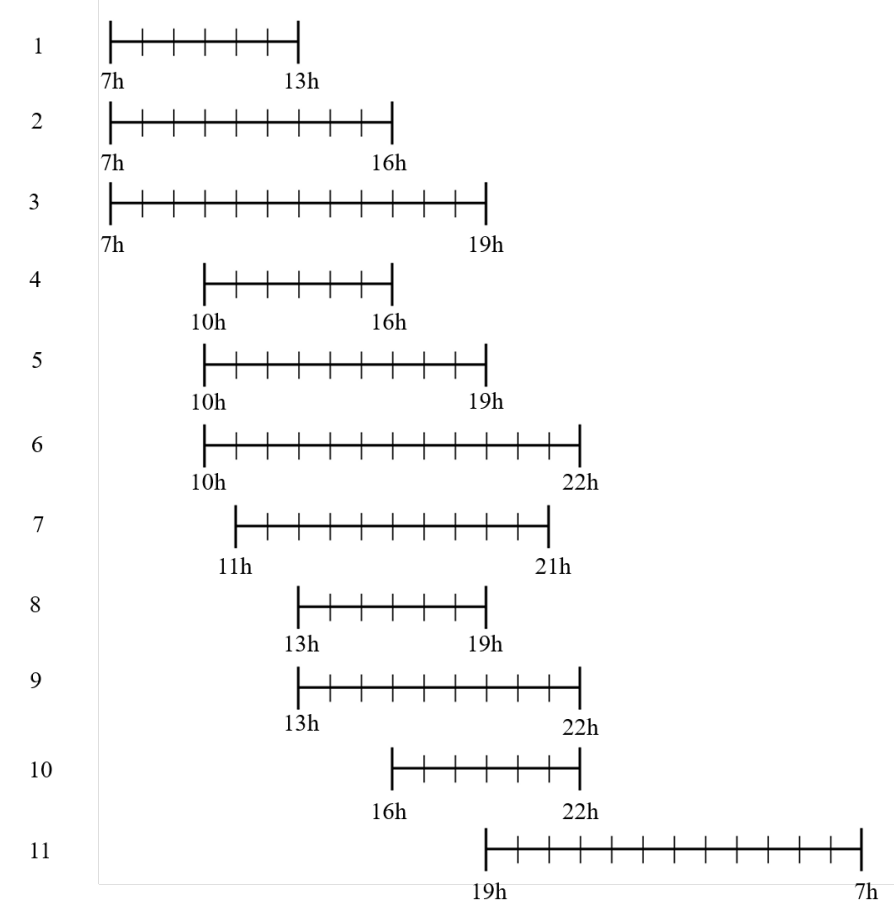

Figure 4.7: Shifts distribution adopted by Clinical Medicine in the ED of Hospital B (24 hour clock format)

Physicians at the hospital are classified as fixed physicians, usually more experienced doctors with longer working hours in the hospital, and oncallers. The former are physicians who must meet a fixed workload in the emergency during the weekdays. The number of hours available in weekday $\left(W L \_W E E K_{p}\right)$ for fixed physicians is $27 \mathrm{~h}$, and each physician must be assigned for each of the shift types $\left(M A X \_T Y P E_{p s}=1\right)$. The shifts types are defined in terms of the duration. The Ed work with shift of $6 \mathrm{~h}, 9 \mathrm{~h}$ and $12 \mathrm{~h}$. Fixed physicians are allocated on a rotational basis on weekends, so each weekend a fixed physician is designated to be on duty (the same doctor is allocated both on Saturday and Sunday) in a shift of type $10 \mathrm{~h}$ $\left(M A X \_T Y P E_{p s}=2\right)$. Hence, the number of hours available in the weekend $\left(W L \_W E E K E N D_{p}\right)$ is $20 \mathrm{~h}$. The on-callers physicians may be allocated to more than one shift per day as long as the shifts are non-overlapped and sequential, and their combined length (in hours) does not surpass the maximum number of allowable consecutive work hours which is $12 \mathrm{~h}\left(M A X \_T Y P E_{p s}=\right.$ $2)$. The total workload for $\left(W L \_T O T A L_{p}\right)$ varies for each physician, being 12 or 24 hours. These physicians can be assigned for both weekdays or weekends. 
Additional scheduling rules and contractual agreements for this groups include: (i) each physician may only be assigned to one shift of type 12h; (ii) overlapped shifts may not be used for combined allocation; (iii) non-overlapped and sequential shifts allow combined allocation.

Of note, a minimum number of physicians must always available in the ED, meaning that parameter $C A P \_M I N_{h t}$ takes the value between 4 and 6. The staffing levels and scheduling defined is repeated weekly in a four-week cycle; thus, the parameter PLAN_HORIZON takes a value of 7 days and parameter $P L A N \_P E R I O D$ also takes the value of 7 days. This last statement, states that this ED run the model once per month (e.g., at the beginning of the month) and uses the same schedule (staffing and scheduling) for the four weeks of the month. In contrast to the case study present previously, in this case study the scheduling is cyclic in the month, i.e., the assignment of the physicians is the same, week by week in a month, since the PLAN_HORIZON and the PLAN_PERIOD takes the same value, so the staffing and the scheduling is cyclic. In the first case study, only the staffing was cyclic being the same week by week in a month, and the assignment of the physician might be different over the weeks.

\subsection{1}

\section{Physician staff}

Currently, analagously to the previous case studied presented, staffing at the ED of Hospital B is performed through an empirical analysis of demand behavior. Generating a manually-defined schedule (shown in Table K.1 on the Appendix K) is a laborious task and its solution is not necessarily optimal. The current schedule's inefficiency reflects in a gap between current levels of demand and capacity at the ED. Figure 4.8 shows average waiting time per hour of the day considering the current schedule in the ED of Hospital A. These are data from the simulation model using the current schedule adopted by this ED. It is clear from Figure 4.8 that the waiting time increase along of the day. This happens beacause there are several points in time when demand greatly exceeds capacity; this occurs because the current schedule does not account for uncertainty in patient arrival. 


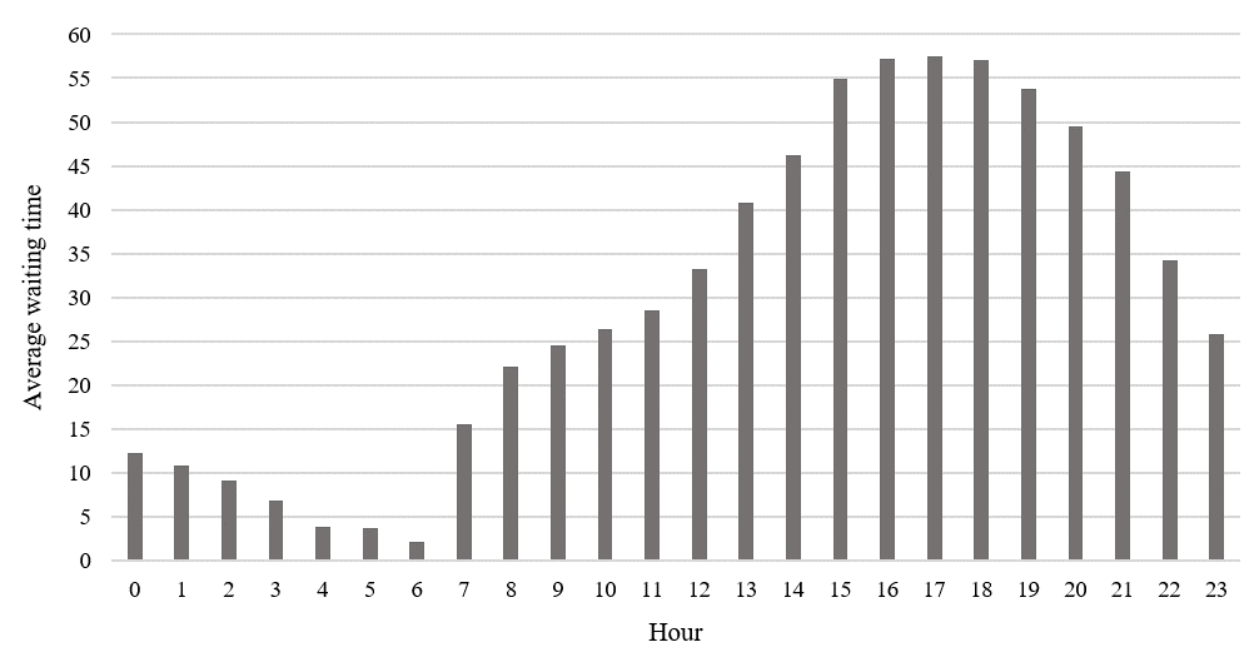

Figure 4.8: Average waiting time per hour in Hospital B

\subsection{2}

\section{Physician scheduling}

ED managers' main concerns also relate to the occurrence and length of the queue, which directly affects a patient's wait time and their total length of stay in the ED. At this point, it is worth mentioning that the current manually-defined schedule (see Table K.1 on Appendix K) fails to address the concerns of ED managers regarding the occurrence and length of patient queues, but, different from the previous case study, do not fail in respect to the several aforementioned scheduling rules since in this case we verify fewer service requirements and contractual agreements, which facilitates the task of distributing doctors by the days and shifts. However, by being manually made, the current schedule does not consider all shift flexibility that would be possible. In Figure 4.7, we show that the physicians can be currently assigned to eleven overlapped shifts, but in fact, the doctors' entry time into the ED could be flexibilized to meet the demand better. If that happened, the different types of shifts used (6h, 9h, 10h, and 12h) in the ED could start any time from 7 am to $7 \mathrm{pm}$ and the number of shifts would be doubled. In this context, the ultimate goal of the physician scheduling phase is to ensure that the available physician workforce is assigned in such a way to minimize patient queue and allow the flexibility of work shifts in the ED, while taking into account all schedule requirements and contractual agreements. 


\subsection{3}

\section{Simulation model}

For the simulation model of the ED of Hospital B, similarly to the simulation model for the previous case study, we assume the premises cited in section 3.2.4 and we consider an estimated service time of 20 minutes as the average for exponential distribution. We assume that the patients arrival follows a Poisson distibutions as showed in the fit test performed using the real data (Figure K.1 in the Appendix K). To define whether the pattern ot patients arrival is diffent over the days of week and the hour of the day, we conducted a Kruskal-Wallis test which results indicated a statistically significant difference between patient interarrival times for each day of the week and hour (KruskalWallis test, chi-squared $=8238.8, \mathrm{df}=23, \mathrm{p}$-value $<2.2 \mathrm{e}-16)$. We used the Kruskal-Wallis test due to the non-normality of the data set (Shapiro test, W $=0.7866$, p-value $<2.2 \mathrm{e}-16)$. Then, we perform a multiple comparison test (results are shown in Table K.2 of Appendix K) to define which hours and days of the week could be considered to have the same arrival rate and, hence be grouped. These tests is performed using the data of patients arrival in the $\mathrm{ED}$ for the period analyzed, as mentioned before we are working with about 73,000 patients.

The current manually-defined ED schedule was used to define the warmup period and number of required replications, and to validate our simulation model. A warm-up period of 10 hours and a total of 100 replications were found to ensure that system occupancy corresponded to real starting conditions. Each replication simulated 6,585 hours of operations, which amounts to the total length of the period for which ED data was made available. Model validation was performed by comparing the real number of patients treated in the ED (72,988 patients) with the number of patients serviced in the simulation model $(72,799$ patients), which yielded a variation of approximately $0.26 \%$ in the total number of treated patients. We considered such variation to be negligible and hence indicative of the simulated model's accuracy

\subsection{4}

\section{Numerical results of the case study in the Hospital B}

We start defining the number of scenarios that should be used in the experiments. We run preliminary tests as specified in Section 3.2.2 using Monte Carlo Sampling (MCS) and Latin Hypercube Sampling (LHS). The size of the models (i.e.,variables and constraints), average execution times, and their variances are shown in Table H.1 of Appendix H. The average solution time of the experiments was 172.99 CPU seconds for 100 scenarios and 2188.666 CPU 
seconds for 1,000 scenarios.

Table 4.7 present the results for each experiment in terms of the objective function for the in-sample and out-of-sample tests and the optimality gap showing the average and standard deviation.

Table 4.7: Experiment results: in-sample and out-of-sample analysis and estimative of the optimality gap

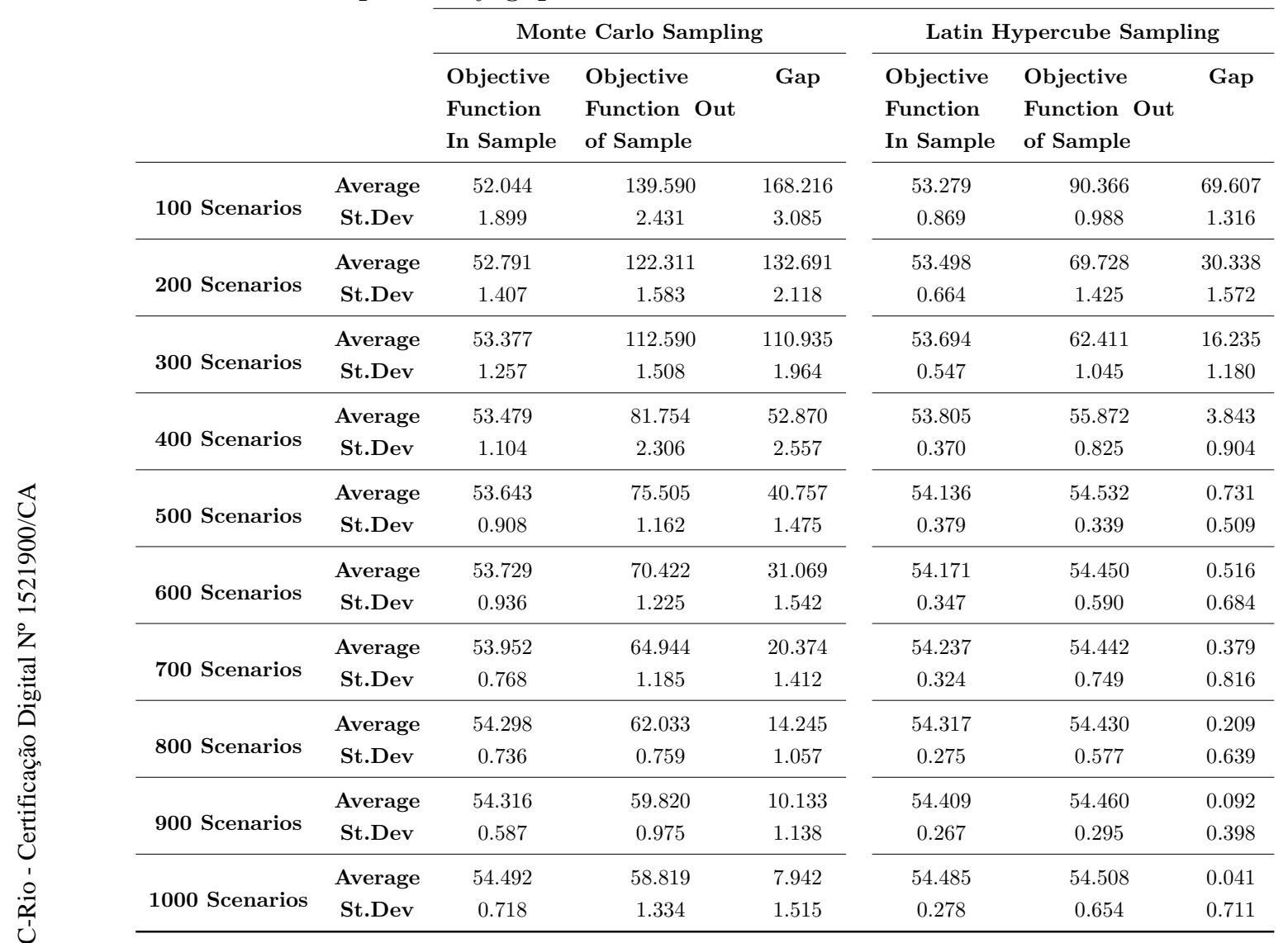

Similar to the previous case study, we observe that using the LHS method we have a faster convergence to a gap $\leq 1 \%$ while using the MCS method even with 1,000 scenarios the gap still large, $\geq 7 \%$. We notice that optimality gap achieve the stop criterion defined for about 500 scenarios using the LHS, being the gap $0.731 \%$ and the $95 \%$ level of confidence interval (CI) is 0.260 , thus the gap considering the $\mathrm{CI}$ is lower than $1 \%$. We show the CI for each experiment in Table I.1 of Appendix I. So all results presented hereafter will be using this scenario setting.

We evaluate the average number of solutions in the in-sample analysis (showed in Tables I.2 and I.3 of Appendix I) and we also evaluate the average number of dominant solutions in the in-sample analysis (showed in Tables I.4 - I.23 of Appendix I). Even the analysis of dominant solutions for the MCS method point that using 1,000 scenarios the average number of solution is close 
to 1.0, the optimality gap analysis does not allow us to affirm that this solution is the true solution of the problem. However, by doing the same analysis using the LHS method we have the hypothesis that we achieve solutions that are close to the real optimal solution of the problem, i.e., the solutions have low gap, within the established criterion, and the number of dominant solutions tends to 1 , so we can consider the true solution for the problem according to SAA technique discussed in the Chapter 3.

At this point, it is worth pointing out that we also perform a preliminary study in the Hospital B using just historical data (HD) as scenarios in the optimization model. This preliminary study is presented in Appendix J. Using the historical data, we could generate just 42 demand scenarios, each of which corresponded to the realization of real ED historical demand.

Table 4.8 contrasts indicators of service quality for the current schedule with those obtained through discrete-event simulation of the optimal schedule generated by model using SAA and using historical data. For results related to the models, in addition to the value of each indicator, we present the percentage variation for: model using historical data vs current schedule $\left(\Delta_{1} \%\right)$; model using SAA vs current schedule $\left(\Delta_{2} \%\right)$; model using SAA vs model using historical data $\left(\Delta_{3} \%\right)$. In addition to the value of each indicator, we also report the $95 \%$ level of confidence interval (CI).

Several points are worthy of mention: first, note the reduction of approximately $28 \%$ in the frequency of queues, which is greater reduction than the one shown when using historical data. Moreover, the frequency of queues with more than 10 patients (one of the main quality indicators defined by managers from the real ED we are analyzing) decreases approximately $62 \%$, which is considerably lower than both the current schedule and the schedule generated using historical data. Additionally, with the model using SAA, we achieve a reduction of approximately $60 \%$ in the number of patients in the queue, while the average time door-to-doctor decreases by approximately $48 \%$ in relation to the current schedule.

Table 4.8: Discrete-event simulation results for case study in the Hospital B using SAA

\begin{tabular}{|c|c|c|c|c|c|c|c|c|c|}
\hline & \multirow{2}{*}{\multicolumn{2}{|c|}{ Current Allocation }} & \multirow{2}{*}{\multicolumn{3}{|c|}{ Model using HD }} & \multirow{2}{*}{\multicolumn{4}{|c|}{ Model using SAA }} \\
\hline & & & & & & & & & \\
\hline & Value & CI & Value & $\mathrm{CI}$ & $\Delta_{1} \%$ & Value & CI & $\Delta_{2} \%$ & $\Delta_{3} \%$ \\
\hline Number of patients & 72798.72 & 60.18 & 72981.01 & 63.36 & $0.25 \%$ & 72818.73 & 65.4 & $0.03 \%$ & $-0.22 \%$ \\
\hline Frequency of queue (\%) & 32.21 & 0.00 & 25.25 & 0.00 & $-21.61 \%$ & 23.12 & 0.00 & $-28.22 \%$ & $-6.61 \%$ \\
\hline Frequency of queue $>10$ patients $(\%)$ & 12.75 & 0.00 & 7.83 & 0.00 & $-38.59 \%$ & 4.79 & 0.00 & $-62.40 \%$ & $-23.81 \%$ \\
\hline Frequency of queue $<=10$ patients (\%) & 19.46 & 0.00 & 17.69 & 0.00 & $-9.10 \%$ & 16.33 & 0.00 & $-16.08 \%$ & $-6.99 \%$ \\
\hline Average number in queue & 3.87 & 0.45 & 2.23 & 0.04 & $-42.45 \%$ & 1.55 & 0.02 & $-59.95 \%$ & $-17.50 \%$ \\
\hline Average door-to-doctor time (min) & 36.55 & 0.45 & 25.02 & 0.47 & $-31.55 \%$ & 18.86 & 0.23 & $-48.42 \%$ & $-16.86 \%$ \\
\hline Total numer of physicians hours used & 1457.00 & 0.00 & 1457.00 & 0.00 & $0.00 \%$ & 1457.00 & 0.00 & $0.00 \%$ & $0.00 \%$ \\
\hline
\end{tabular}

Figure 4.9 shows the comparison of the current empirically-defined 
schedule of ED in the Hospital A and the schedule from the proposed model in terms of service demand and capacity. From the Figure, we observed that with the schedule from the proposed model, we were able to better adapt the capacity to the demand of the day achieving better waiting times.

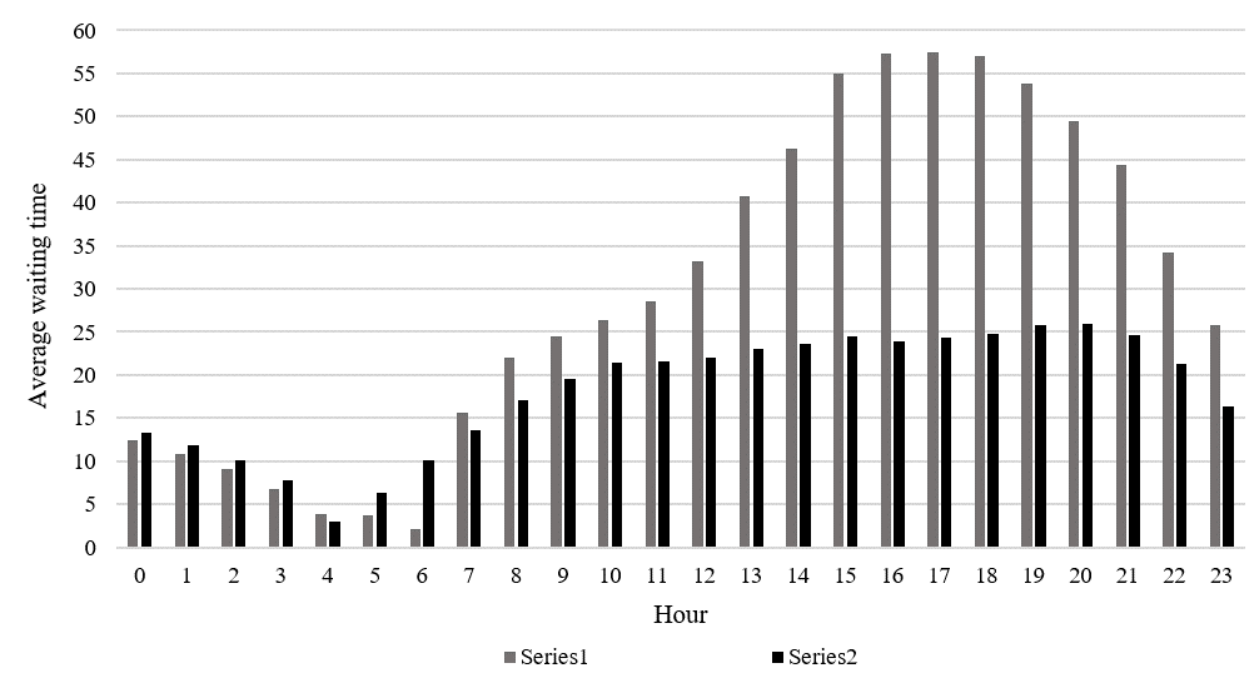

Figure 4.9: Average waiting time in Hospital B comparing the current empirically-defined schedule of ED in the Hospital B and the schedule from the proposed model

Figure 4.6 presents the histograms of idle capacity and queue frequency for the current schedule and the schedule generated by Model using SAA. As shown in Figure 4.10, we observe that queue using the scenarios generation are even smaller than that presented in J.1(b) and also less frequent. 


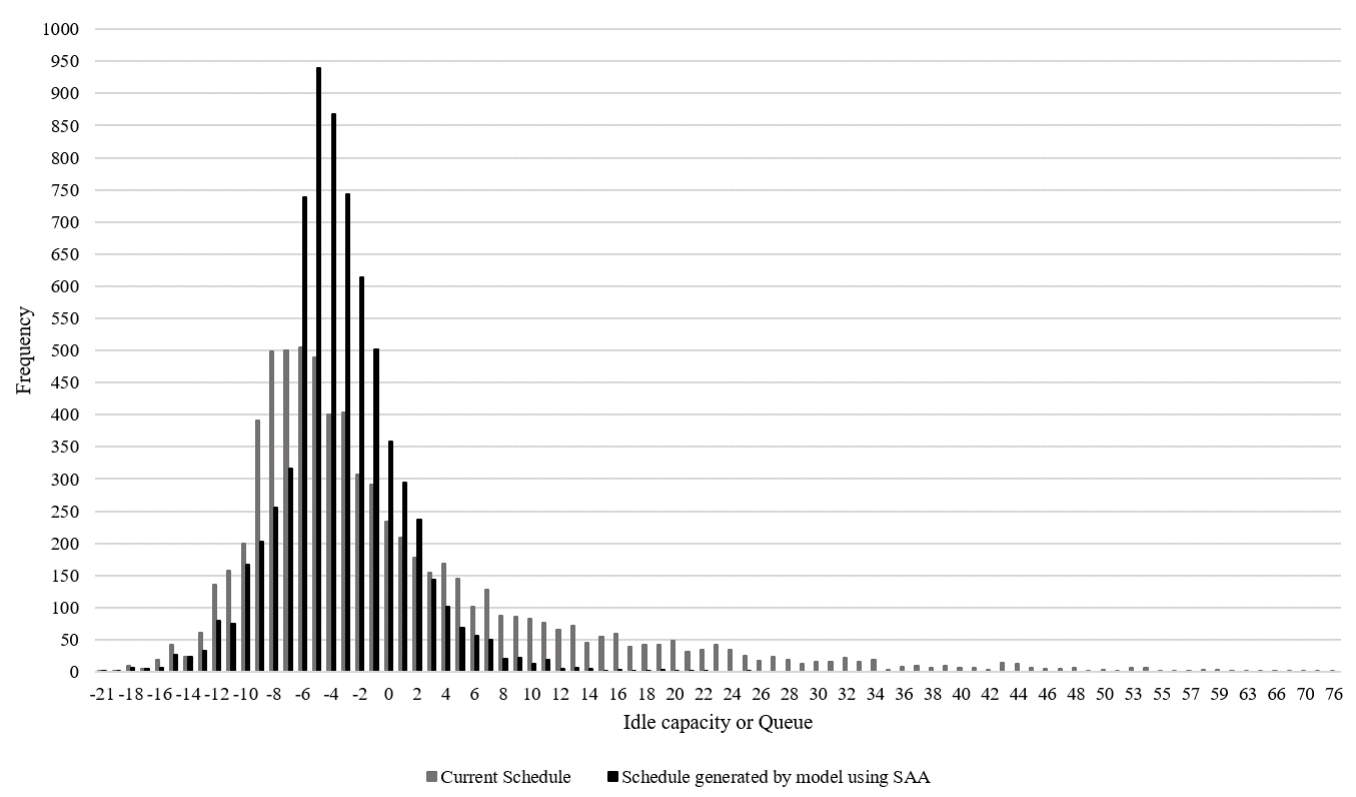

Figure 4.10: Histogram of frequency distribution of idle capacity (negative values) or queue (positive values) for the current schedule and the schedule generated by model using SAA

Taken together, these results indicate that the schedule generated by model using SAA takes advantage of the wide representation of the uncertainty in the arrival of patients by the generation of scenarios and thus achieves better results than those achieved using only historical data.

\section{7}

Discussion

The methodology we propose is able to optimally generate a schedule that aligns physician service capacity with patient arrivals in the ED because an optimal staffing level is defined while accounting for all schedule requirements and contractual agreements, i.e., staffing and scheduling are performed in an integrated manner. Our results show improvements in ED performance indicators such as frequency of queue and average door-to-doctor time. Moreover, our methodology is generalizable to different ED characteristics, such as shift configurations, number of physicians, category of physicians, cyclic and acyclic approaches to staffing and scheduling, among others.

Both case studies performed attest to the importance of accounting for differences in patient arrival rates by day and hour to better assign physicians throughout the week. In addition, allowing for flexibility in physician entry time was also found to contribute to a better alignment between demand and 
capacity. An essential feature of our model is that it is capable of considering individual physician preferences and/or restrictions in terms of the start time in the ED $\left(S T A R T \_C O M B_{s h p}\right)$ and availability $\left(I \_P H Y S I C I A N_{s d p}\right)$. These characteristics, in association with complex shift configurations (e.g., those shown in Figures 4.3 and 4.7), and several contractual agreements and service requirements, make it necessary to perform the staffing and scheduling phases in an integrated manner. In fact, under these conditions, it is not possible to generate a feasible schedule for an established staffing level if the two phases are performed separately.

In our first case study, cyclic staffing and acyclic scheduling were performed, while in the second case study, cyclic staffing and scheduling were performed. In both Hospitals A and B, staffing is cyclic to guarantee the same service level over the weeks. In terms of scheduling, Hospital A performs scheduling in an acyclic manner over a period of 4 -week because each physician has a certain total numbers of hours available and a number of hours available to weekends for the 4-week period, but this number of hours available can be distributed differently over the weeks. Moreover, in Hospital A physicians define their preferences for a 4-week cycle which can also be different over the weeks. In Hospital B, physicians define their preferences for a period of 4 weeks, but these cannot change over the weeks, and each physician is required to perform the same number of hours per week, thus the scheduling in this hospital is cyclic. Hence, it is clearly important to be able to use a general model capable of performing both cyclic and acyclic staffing and scheduling, considering that real needs vary from hospital to hospital, and our model was shown to be adaptable to these different ED needs.

We can see from the results that although Hospital B has more physicians available, the result in terms of proportion of queue reduction and waiting time is shorter. This is because despite more doctors, the total hours available from these doctors is lower than observed for Hospital A. Hospital A has 44 physicians available with a total of 3,888 hours while Hospital B has 85 physicians available and a total of 1.457 hours. A feature of Hospital A is that physicians dedicated exclusively to the hospital, but at Hospital B this doesn't happen, so with more doctors we still have fewer hours available. Another important observation is the fact that Hospital A has more flexibility regarding the allocation of doctors during the planning horizon. This means that doctors can be allocated to any shift on weekdays or weekends, except for the personal restrictions set by each physician. This added to the flexibility of the entry time give to the proposed model much more freedom for optimization which leads to a greater gain in the system. Hospital B, on the other hand, has no 
flexibility regarding the allocation of doctors by shifts and days of the week, being the only flexibility related to the entry time.

Taken together, results indicate that the schedule generated by our model using SAA takes advantage of the wide representation of the uncertainty in the arrival of patients by scenario generation, and thus achieves better results than those achieved using only historical data. In addition, we observe that Latin Hypercube Sampling (LHS) converged faster in SAA for this type of problem. This result corroborrates to the results shown in Homem-de Mello et al. [2011]. [?] show that for larger problems it is important not only the definition of efficient stopping criterion but also use a more efficient alternative sampling techniques, like the LHS.

The main limitation of our study deals with the fact that we assumed fixed and constant physician service rates across the entire planning horizon. Nevertheless, as shown in Kuo [2014], this assumption is acceptable in an ED setting because emergency physicians are cross-trained (i.e., equipped with all essential medical knowledge for potential illnesses and injuries of patients in EDs) and must work as efficiently as possible. Another important point is that we are not taking into account the patient's case mix to vary the physician's workload according to the complexity of the patients. These two points are potential issues to be addressed in future work.

\section{8 \\ Conclusions}

In sum, the methodology we propose can optimally generate a schedule that aligns physician service capacity with patient arrivals in the setting of two Brazilian hospitals, while accounting for all schedule requirements and contractual agreements. Aligning physician schedule with patient demand is a strategy for improving patient throughput in the ED. In the following chapter, we present an extension of the problem presented in this chapter to deal with the points considered by the works in the literature related with this study and do not addressed here (points showed in the two last rows of Table 4.1). 


\section{Resources Staffing and Scheduling to Reduce Waiting Time in an Emergency Department}

In this chapter, we address the problem of the Physician Staffing, and Scheduling in an Emergency Department (PSSED) presented earlier in Chapter 4. At that time, we were interested just in the door-to-doctor time, i.e. we addressed just the first assessment, here we consider the entire clinical pathway that a patient can follow in an ED. For this, we include the dynamics of the patient flow through the ED. It is worth mentioning that we would still need the model presented in Chapter 4. The reason is that not every hospital has sophisticated information systems that record every stage of the care delivery process, making it difficult to apply the extended model to their case.

Figure 5.1 represents the overall flow in an ED with the key treatment steps (prevalent in most EDs). 


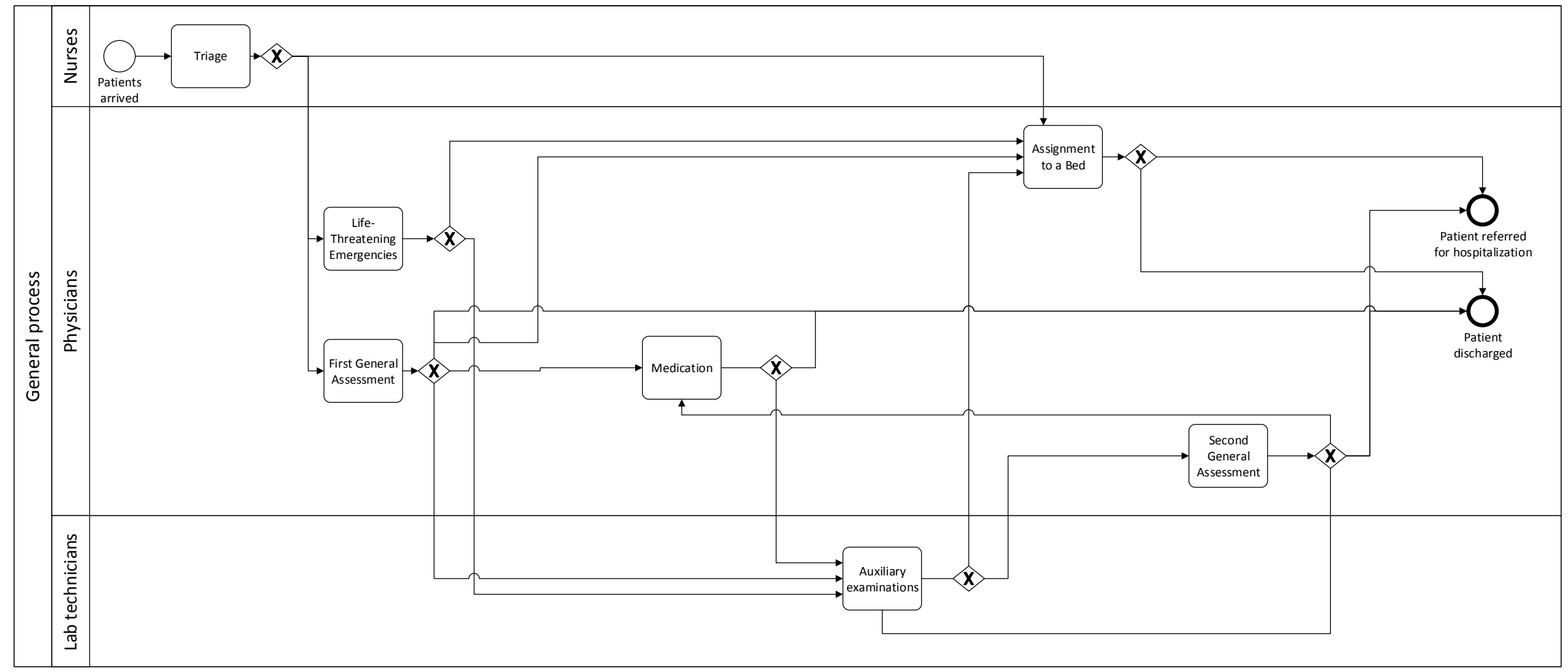

Figure 5.1: Complete flow that represents the process in the ED

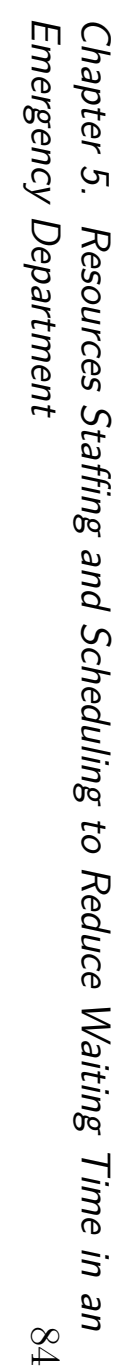


In Figure 5.1, patients may follow different paths in an ED; these paths vary from case to case and how each case is treated in each ED. The process begins when patients arrive in the ED. Usually, the patients go to a nurse (to the triage) who determines whether they need vital medical care. From here, we can distinguish three possible cases: (i) patients who do not need vital care but require special conditions of treatment go directly to a bed in the ED; (ii) patients who need vital care are treated in the Life-Threatening room; (iii) patients who do not need vital care stay in a waiting room until a physician from Clinical Medicine (CM) is available to do the First General Assessment.

Patients from case (ii) may go to a Bed after the encounter in the LifeThreatening room or after doing Auxiliary Examinations; and Patients from case (iii), after the first encounter, may pass through Medication (where they occupy a chair), Auxiliary Examinations, Second Assessment or go to a Bed.

The three types of health care providers that we observe in the Figure 5.1 are physicians, nurses, and lab technicians. From the flow explain above, we can define that: physicians perform health assessments before and after having auxiliary examinations, examine patients in the Medication phase, examine patients in the Life-Threatening room and in the Beds; nurses handle the triage of patients; lab technicians handle the auxiliary examinations. In this chapter, we consider two main categories of human resources in an ED that are, the physician and nurses. The lab technicians are out of scope since they are often resources not entirely dedicated to the ED. Also, we consider a limit for the main material resources, chairs in the medication phase, and beds in the assignment to a bed phase respectively, which are considered critical resources in ED [Daldoul et al., 2018]. Each activity in the Figure 5.1 represents a treatment stage in the ED. The activities described in the doctor's row can be performed by any physicians that are allocated in that shift and belonging to the category eligible for that treatment step.

The extended PSSED presented here follows the same assumptions described in Chapter 4. Thus, the PSSDE is performed for a planning horizon that consists of a set of subsequent days of finite and fixed length $D$. In our model, the planning horizon can be divided into $T$ periods over which the staffing level will be repeated. We assume that a fixed number of physicians $P$, belonging to $K$ different skill categories, must be scheduled over the planning horizon. Each day in the planning horizon consists of $H$ hours and is divided in $S$ shifts that may overlap. Moreover, each shift is associated with a specific workload, defined in terms of the shift's start time and duration. Each physician is scheduled for a certain number of hours during the entire planning horizon, but differing between weekday and weekend. The 
physician scheduling should respect a minimum interval between assignments. Eventually, when the physician is allocated for the night shift, the interval between assignments should be greater than when the physician is assigned to other shifts. Furthermore, physician scheduling should respect the maximum allowed consecutive hours of work. It is required that a minimum number of physicians is always available in each stage of treatment in the ED. The patients may pass through several phases $Q$ depends on their clinical pathway. Here, we also consider that the patients arrival varies per day and hour. Besides, we assume that the demand is uncertainty, and this characteristic should be considered into the model so that the decisions are properly taken, considering different possibilities for these uncertain events.

To address this issue, we propose a two-stage stochastic programming formulation with fixed recourse. The mathematical model's objective is to minimize the total number of patients waiting per hour, considering all stages of the treatment, while taking into account scheduling requirements and contractual agreement for the physicians. Therefore, we seek to determine the number of staff (physicians and nurses) required to be in service during each shift, for each day of the planning horizon, and each stage of the treatment. Also, we want to assign individual physicians to each day and shift in accordance with service requirements and contractual agreements, taking into consideration uncertainty in patient arrival patterns. We consider this problem as an integrated model able to perform both staffing and scheduling phases together.

As mentioned above, this is an extent of the problem Physician Staffing, and Scheduling in an Emergency Department (PSSED) addressed in Chapter 4, thus here we address the points that we did not cover at that time. Therefore, here, we will consider as in the work of [Daldoul et al., 2018; EL-Rifai et al., 2015] several stages of treatment in an ED and other resources than physicians. Daldoul et al. [2018] deal with three phases of treatment in an ED, and consider physicians and nurses in the study; EL-Rifai et al. [2015] extend the approach to contemplate beds and other stages of treatment in an ED (six steps of treatment). Here, we address seven stages of treatment in an ED in addition to accounting for nurse staffing, physician staffing, and scheduling, and considering a limit for chairs and beds. In addition, the contributions described previously still apply to the problem dealt with here. The Table 5.1 updates the Table 4.1 from Chapter 4 . 
Table 5.1: Summary of contributions from the Chapter 5

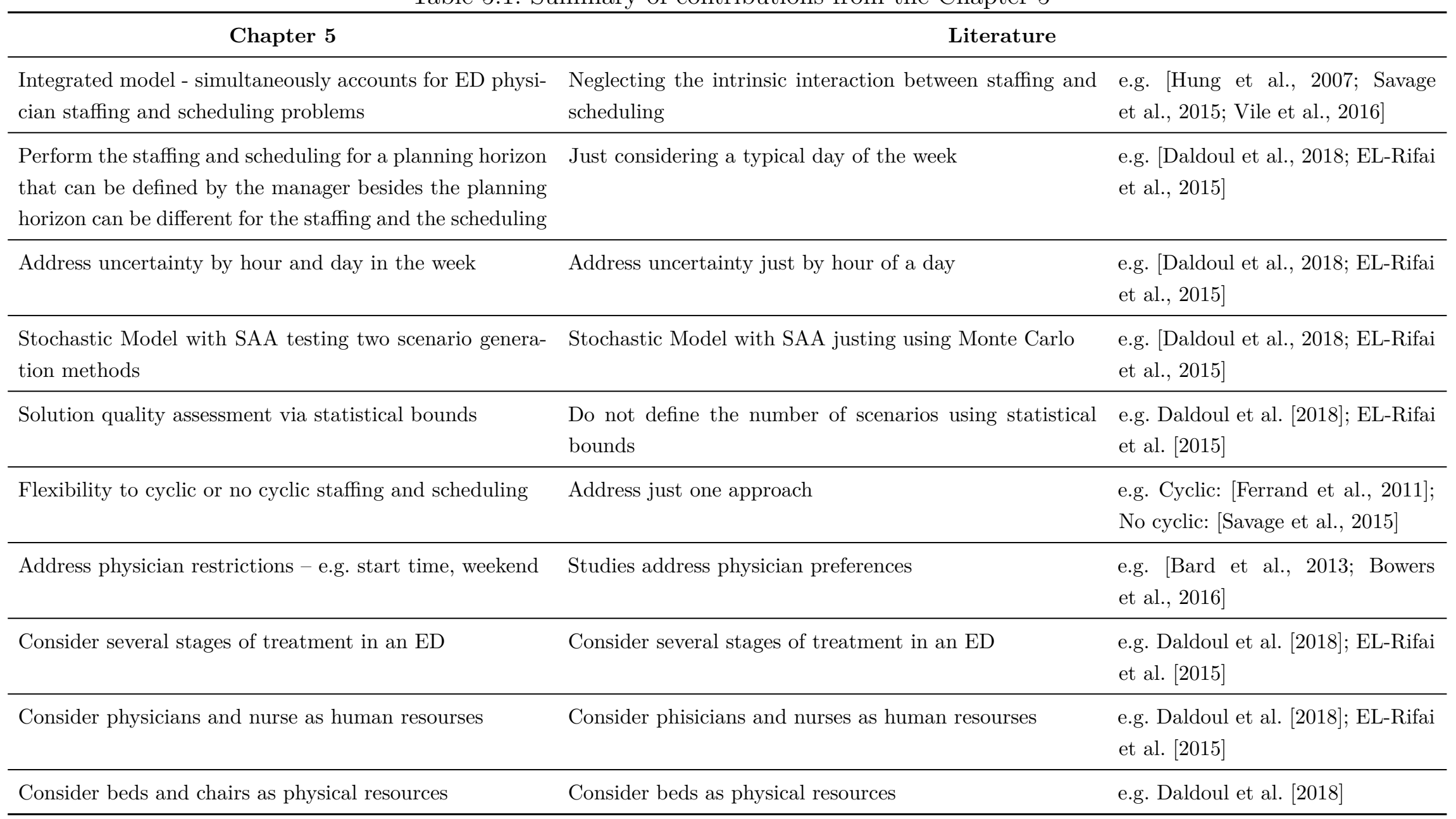




\section{1}

\section{General Mathematical Model}

In this section, we present the mathematical model developed to deal with the problem mentioned above. The objective of this mathematical model is to minimize the expected value of the total number of patients waiting.

The first-stage decisions (assumed to be made before the realization of random variables, i.e., previously to the unveiling of the uncertainties) define the number of nurses and physicians that are required to each day and shift and define the physicians to be assigned to meet the established staffing level. These decisions are represented by integer and binary variables, respectively.

Second-stage decisions are taken under complete information of the uncertainty, that are: number of patients waiting in a queue and number of patients served in a queue. We assume that uncertainties (patients arrivals) are discretely represented by $S C$ possible realization scenarios. We further adopt that the probability of scenario $c$ occurring is represented by a scenario-dependent parameter $P R O B_{c}$, defined such that $P R O B_{c} \geq 0$ and $\sum_{c=1}^{S C} P R O B_{c}=1$. We assume that the scenarios have the same probability of occurrence.

From Chapter 4, we have seen that the flow of patients into an ED is represented by a queuing system consisting of treatment steps and resources. Here, each treatment step can also be seen as a queuing system. In this section, we represent the problem described in the previous section by a queue system showed in Figure 5.2. 


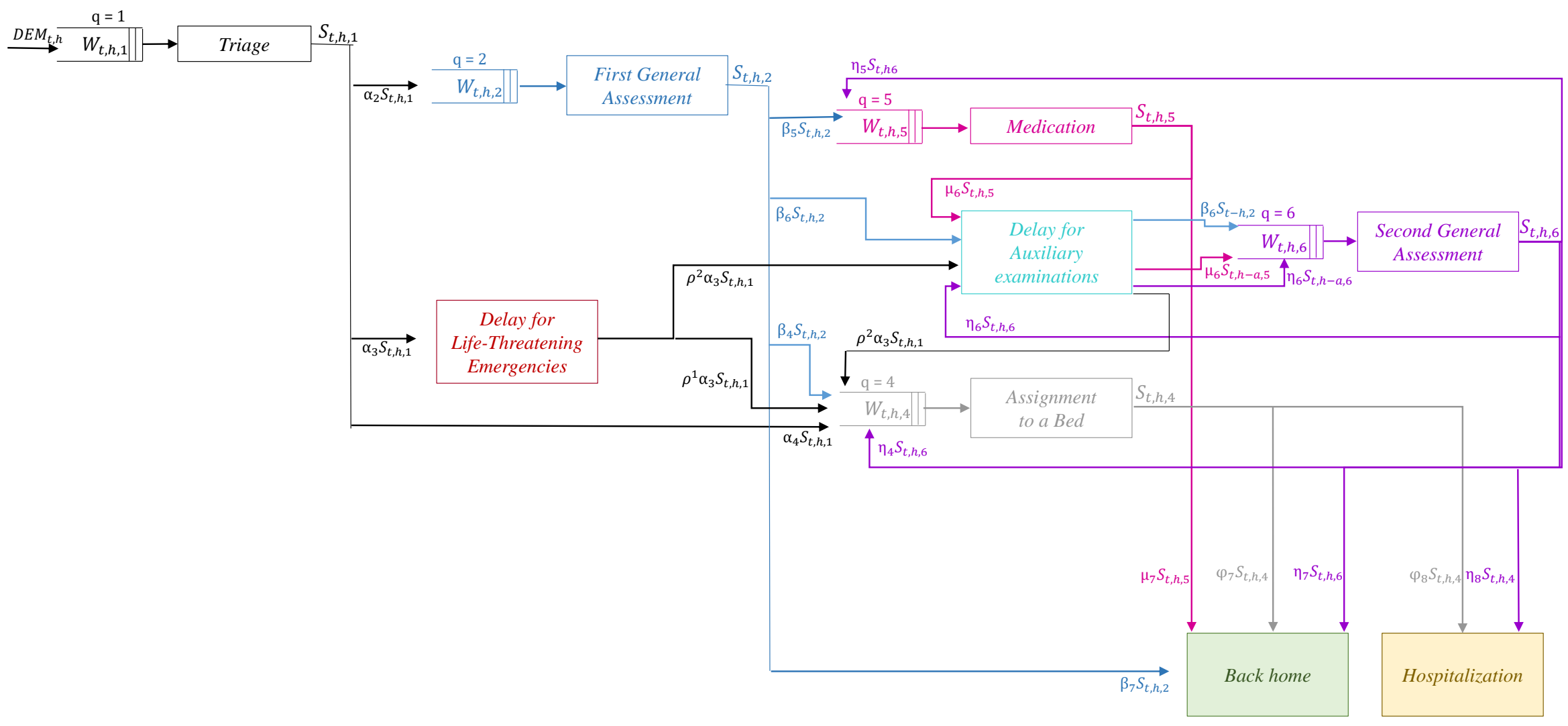


The mathematical model presented in this section is based on the problem described in the previous section and represented by Figure 5.2. The number of patients arriving in the system at each period $t$ an hour $h$ is denoted $D E M_{h t}$. After the patients enter in the system, they are counted as waiting by the variable $W_{h t q}$. Patients that are served in a queue are counted by the variable $S_{h t q}$. The number of patients served in a queue and which are transferred to another queue depends on the transfer rate $\left(\alpha_{q}, \beta_{q}, \eta_{q}, \mu_{q}, \rho^{1}, \rho^{2}\right)$. In this section, queue and stages of treatment are used as synonyms.

We made some assumptions, following the definitions made by Daldoul et al. [2018] and EL-Rifai et al. [2015], to solve the problem more efficiently:

- The system is initially empty. For this, we start the scheduling horizon at an hour when the system is usually empty [Daldoul et al., 2018; EL-Rifai et al., 2015];

- The waiting time of patients during the period they arrive is not counted [EL-Rifai et al., 2015];

- We do not perform scheduling of patients (scheduling of patients is object to some studies e.g. Bagwandeen and Carroll [2013]). Because of this, the patients are divided into three possible cases (as we explained in the previous Section) and each case has a well-defined flow from the beginning of the treatment.

- We consider the physicians from the same skill categories and the nurses to be homogeneous, i.e., we do not differentiate between them [EL-Rifai et al., 2015];

- The patients who need auxiliary exams are just delayed because we do not consider a staff for this stage of treatment, i.e., this phase of treatment is considered to have infinite capacity, and we do not consider queue for this phase and consequently we do not create variables related to these treatment stages. Thus auxiliary examinations are always assumed to have a fixed duration [EL-Rifai et al., 2015].

- The patients who need treatment in the life-threatening emergency room are just delayed because we do not consider staff for this stage of treatment. This is because the physicians are mostly not dedicated to this phase of the treatment. Thus treatment in the life-threatening emergency room is always assumed to have a fixed duration.

Some of this assumptions are relaxed in the simulation model presented later in Section 5.2. 
Table 5.2 presents the domains in which our model attributes are defined, while Tables 5.3 and 5.4 provide a complete listing of parameter and variable definitions.

Table 5.2: Sets, subsets and corresponding domains of the general model

\begin{tabular}{|c|c|c|c|}
\hline Sets & Indexes & Domain & Description \\
\hline Shifts $(S)$ & s, s' & $\{1, \ldots,|\mathrm{S}|\}$ & Shifts in the ED \\
\hline Physicians $(P)$ & $\mathrm{p}$ & $\{1, \ldots,|\mathrm{P}|\}$ & Physicians in the ED \\
\hline Days $(D)$ & $\mathrm{d}$ & $\{1, \ldots$, PLAN_HORIZON $\}$ & Days of the planning horizon \\
\hline Hours $(H)$ & $h, h$ ' & $\{0, \ldots, 23\}$ & Hours of the day (24 hour clock format) \\
\hline Period $(T)$ & $\mathrm{t}$ & $\left\{1, \ldots, P L A N \_P E R I O D\right\}$ & Period over which staffing level is repeated \\
\hline Physician Category $(K)$ & $\mathrm{k}$ & $\{1, \ldots,|\mathrm{K}|\}$ & Physician skill categories \\
\hline $\begin{array}{l}\text { Stages of treatment } \\
\text { (queue) }(Q)\end{array}$ & $q$ & $\{1, \ldots,|\mathrm{Q}|\}$ & Stages of treatment (queue) in the ED \\
\hline Scenarios $(S C)$ & $\mathrm{c}$ & $\{1, \ldots,|\mathrm{SC}|\}$ & Possible realization scenarios \\
\hline $\begin{array}{l}\text { Physician in certain cate- } \\
\text { gory }\left(P_{k}\right)\end{array}$ & - & Subset of Physicians $(P)$ & Physicians who belong to category $k$ \\
\hline Weekdays $(W D)$ & - & Subset of Days $(D)$ & Weekdays on the planning horizon \\
\hline Weekends $(W)$ & - & Subset of Days $(D)$ & Weekend days on the planning horizon \\
\hline Night_Shifts $(N I G H T)$ & - & Subset of Shifts $(S)$ & Night shifts \\
\hline N_Shifts $(N S)$ & - & Subset of Shifts $(S)$ & Shifts that do not allow combined allocation \\
\hline Y_Shifts $(Y S)$ & - & Subset of Shifts $(S)$ & Shifts that allow combined allocation \\
\hline Physicians_Week $(P 1)$ & - & Subset of Physicians $(P)$ & Physicians that work only week days \\
\hline Physicians_Both $(P 2)$ & - & Subset of Physicians $(P)$ & Physicians that work both week and weekend days \\
\hline
\end{tabular}


Table 5.3: General model parameters

\begin{tabular}{|c|c|c|}
\hline Parameters & Description & Unit \\
\hline $\mathrm{PROB}_{c}$ & Probability of each scenario $c$ & - \\
\hline PLAN_HORIZON & Number of days in the planning horizon & - \\
\hline$P L A N \_P E R I O D$ & Period (number of days) over which the staffing level is repeated & - \\
\hline$D U R_{s}$ & Duration of each shift $s$ & Hour \\
\hline$W L \_W E E K_{p}$ & Number of hours available for each physician $p$ in the week & Hour \\
\hline$W L \_W E E K E N D_{p}$ & Number of hours available for each physician $p$ in the weekend & Hour \\
\hline$W L_{-} T O T A L_{p}$ & Total Number of hours available for each physician $p$ & Hour \\
\hline$M A X \_A S S I G N 1$ & Maximum number of assignments per day & - \\
\hline$R E L \_S H I F T S_{s s^{\prime}}$ & Indication if shift $s$ can be combined with shift $s^{\prime}$ & $\{0,1\}$ \\
\hline$M A X \_A S S I G N 2$ & Maximum number of assignments per day & - \\
\hline$M A X \_T Y P E_{p s}$ & Maximum number of assignments in a certain shift type & Hour \\
\hline$R E L \_P H Y S H I_{p s}$ & Indication if physician $p$ can be schedule for shift $s$ & $\{0,1\}$ \\
\hline$S T A R T \_C O M B_{s h p}$ & Indication of start time $h$ of each shift $s$ for each physician $p$ & $\{0,1\}$ \\
\hline$M I N_{k}$ & Minimum number of physicians required for each skill category $k$ & - \\
\hline$D A Y \_P E R I O D_{d t}$ & Indication if day $d$ is included in period $t$ & $\{0,1\}$ \\
\hline$D E M_{h t}^{c}$ & Demand (arrival of patients for each period $t$, hour $h$, and scenario $c$ ) & - \\
\hline$\alpha_{q}$ & Transfer rate of patients from the Triage to the stage of treatment $q$ & {$[0,1]$} \\
\hline$\beta_{q}$ & $\begin{array}{l}\text { Transfer rate of patients from the First General Assessment to the stage } \\
\text { of treatment } q\end{array}$ & {$[0,1]$} \\
\hline$\eta_{q}$ & $\begin{array}{l}\text { Transfer rate of patients from the Second General Assessment to the } \\
\text { stage of treatment } q\end{array}$ & {$[0,1]$} \\
\hline$\mu_{q}$ & $\begin{array}{l}\text { Transfer rate of patients from the Medication to the stage of treatment } \\
q\end{array}$ & {$[0,1]$} \\
\hline$\rho^{1}$ & Transfer rate of patients from the Life-Threatening room direct to Beds & {$[0,1]$} \\
\hline$\rho^{2}$ & $\begin{array}{l}\text { Transfer rate of patients from the Life-Threatening room to auxiliary } \\
\text { examinations }\end{array}$ & {$[0,1]$} \\
\hline$M A X \_R_{h t}$ & Maximum number of registration staff available per hour $h$ and day $t$ & - \\
\hline$M A X \_P H Y_{h t q}$ & $\begin{array}{l}\text { Minimum number of physician required per hour } h \text {, day } t \text { and stage of } \\
\text { treatment } q\end{array}$ & - \\
\hline$N_{c}$ & Number of chairs available to treatment in Medications step & - \\
\hline$N_{b}$ & Number of beds available to treatment in Beds room & - \\
\hline$C A P_{h t q}$ & Capacity (service rate per physician) & - \\
\hline$I \_P H Y S I C I A N_{s d p}$ & Indication if physician $p$ is available on day $d$ and shift $s$ & $\{0,1\}$ \\
\hline
\end{tabular}

Table 5.4: General model variables

\begin{tabular}{lll}
\hline Variables & Description & Domain \\
\hline$W_{h t q}^{c}$ & Number of patients waiting in each hour $h$, each period $t$, each queue $q$ and & $\mathbb{R}_{+}$ \\
& each scenario $c$ & \multirow{2}{*}{0,1} \\
$x_{s d p}$ & Decision if physician $p$ is assigned in the shift $s$ in the day $d$ & \\
$n P h y_{h t q}$ & Number of physicians required for each hour $h$, each period $t$ and stage of & $\mathbb{Z}_{+}$ \\
& treatment $q$ & \\
$S_{h t q}^{c}$ & Number of patients served in each hour $h$, each period $t$, each stage of & $\mathbb{R}_{+}$ \\
& treatment $q$ and each scenario $c$ & \\
$n N u r_{h t}$ & Number of nurses required for each hour $h$, and each period $t$ & $\mathbb{Z}_{+}$ \\
\hline
\end{tabular}

In the model, we perform the physicians staffing for each queue on a day and shift, but the scheduling is not specific to a treatment phase, i.e., the model ensures that the number of physicians allocated in the ED each day and 
shift cover the demand. However, these physicians can change between stages of treatment.

The objective of the model is to minimize the total number of patients waiting, i.e., patients in queue. Our model can be adapted to reflect the operational characteristics of several EDs and/or considering other stages of treatment by making minor changes. The proposed mathematical formulation is as follows:

$$
\operatorname{MinZ}=\sum_{h} \sum_{t} \sum_{c} P R O B_{c} W_{h t q}^{c}
$$

The objective function in (5.1) consists of minimizing the total number of patients waiting per hour.

Subject to:

$$
\begin{gathered}
\sum_{d \in W D} \sum_{s} x_{s d p} D U R_{s} \leq W L \_W E E K_{p} \quad \forall p \in P 1 \\
\sum_{d \in W} \sum_{s} x_{s d p} D U R_{s} \leq W L \_W E E K E N D_{p} \quad \forall p \in P 2 \\
\sum_{d} \sum_{s} x_{s d p} D U R_{s} \leq W L \_T O T A L_{p} \quad \forall p \in P
\end{gathered}
$$

Constraints (5-1)-(5-3) ensure that the maximum allocation time for each physician in the planning horizon is respected. Constraint (5-1) ensures that the sum of the shift durations to which the physician has been assigned on week is less than or equal to the total allowable number of hours for week, this for physicians that work only on weekdays. Constrains (5-2) enforces that the sum of the shift durations to which the physician has been assigned on weekends is less than or equal to the total allowable number of hours for weekend. Constraint (5-3) enforces that the total number of hours assigned in the entire planning horizon is less than or equal to the total number of hours allowable.

$$
\begin{aligned}
& \sum_{s} x_{s d p} \leq M A X_{-} A S S I G N 1 \\
& \forall p \in P, \forall d \in D
\end{aligned}
$$

Constraint (5-4) guarantees that each physician is allocated to a maximum number of shifts per day.

$$
\begin{aligned}
& x_{s d p}+x_{s^{\prime} d p}\left(1-R E L \_S H I F T S_{s s^{\prime}}\right) \leq M A X \_A S S I G N 1 \\
& \forall p \in P, \forall d \in D, \forall s \in Y S, \forall s^{\prime} \in S \mid s \neq s^{\prime}
\end{aligned}
$$


Constraint (5-5) ensure that, for any given day, physicians are not assigned to any of the following: shifts that do not respect the minimum interval between assignments; shifts that do not respect the maximum allowed consecutive hours of work; overlapped shifts.

$$
\begin{aligned}
& x_{s d p}+\sum_{s^{\prime} \mid s^{\prime} \neq s} x_{s^{\prime} d p} R E L \_S H I F T S_{s s^{\prime}} \leq M A X \_A S S I G N 2 \\
& \forall p \in P, \forall d i n D, \forall s \in Y S \wedge \exists s^{\prime}
\end{aligned}
$$

Constraint (5-6) allows physicians to be assigned to different shifts on the same day, as long as the shifts are non-overlapped and sequential and the physician belongs to the group of physicians who can work on more than one shift per day.

$$
\begin{aligned}
& \sum_{s \in N i g h t} x_{s d p}+x_{s^{\prime}, d+1, p} \leq M A X \_A S S I G N 1 \\
& \forall p \in P, \forall d \in D, \forall s^{\prime} \in S
\end{aligned}
$$

Constraint (5-7) enforces that physicians allocated on the night shift will not be allocated to any other shift on the same day and can only be allocated on the night shift of the following day, thus guaranteeing that the minimum interval between assignments is respected in the event of physicians being allocated on night shifts.

$$
\begin{aligned}
& \sum_{d} x_{s d p} \leq M A X \_T Y P E_{p s} \\
& \forall p \in P, \forall s \in S \mid R E L \_P H Y S H I_{p s}
\end{aligned}
$$

Constraint (5-8) guarantees that during the planning horizon, each physician is allocated at most the number of times allowed for each type of shift.

$$
\begin{aligned}
& \sum_{p i n P_{k}} \sum_{d} \sum_{h^{\prime}} x_{s d p} S T A R T \_C O M B_{s h^{\prime} p} \geq M I N_{k h} \\
& \forall h \in H, \forall d \in D, \forall k \in K
\end{aligned}
$$

Constraint (5-9) ensures that the minimum number of physicians per hour required for each physician category will be met. 


$$
\begin{aligned}
& \sum_{p} \sum_{d} \sum_{h^{\prime}} x_{s d p} S T A R T \_C O M B_{s h^{\prime} p} \geq \sum_{q} n P h y_{h t q} \\
& \forall h \in H, \forall d \in D, \forall t \in T \mid D A Y \_P E R I O D d t
\end{aligned}
$$

Constraint (5-10) enforces that the number of physicians allocated (by scheduling variable $x_{s d p}$ ) meets hourly the staffing levels defined for physicians (by staffing variable $n P h y_{h t q}$ ).

Constraints (5-11) limit the number of patients served in the Triage phase.

$$
\begin{aligned}
& S_{h t q}^{c} \leq n N u r_{h t} C A P_{h t q} \\
& \forall h \in H, \forall t \in T, \forall q \mid q=1, \forall c \in S C
\end{aligned}
$$

Constraints (5-12) limit the number of patients served in the phases handle by the physicians.

$$
\begin{aligned}
& S_{h t q}^{c} \leq n P h y_{h t q} C A P_{h t q} \\
& \forall h \in H, \forall t \in T, \forall q \mid(q=2 \vee q=4 \vee q=5 \vee q=6), \forall c \in S C
\end{aligned}
$$

Constraints (5-13) specify a bound for the number of patients served during a period.

$$
\begin{aligned}
& S_{h t q}^{c} \leq \text { wait }_{h t q}^{c} \\
& \forall h \in H, \forall t \in T, \forall q \in Q, \forall c \in S C
\end{aligned}
$$

Constraints (5-14)-(5-15) limit the number of patients served in the Medication and Beds, respectively, according to the number of chairs and beds available.

$$
\begin{aligned}
& S_{h t q}^{c} \leq N_{c} \\
& \forall h \in H, \forall t \in T, \forall q \mid q=5, \forall c \in S C \\
& S_{h t q}^{c} \leq N_{b} \\
& \forall h \in H, \forall t \in T, \forall q \mid q=4, \forall c \in S C
\end{aligned}
$$

Constraint (5-16) limits the total number of nurses available to serve the first queue. 


$$
\begin{aligned}
& n N u r_{h t} \leq M A X \_R_{h t} \\
& \forall h \in H, \forall t \in T
\end{aligned}
$$

Constraint (5-17) guarantee the minimum number of physicians required in each queue.

$$
\begin{aligned}
& n P h y_{h t q} \geq M I N_{-} \text {PHYhtq } \\
& \forall h \mid h \geq 1, \forall t \in T, \forall q \in Q
\end{aligned}
$$

Constraints (5-18)-(5-22) update the number of patients in each queue at hours and day.

Triage Queue

$$
\begin{aligned}
& W_{h t^{\prime} q}^{c}=W_{h-1, t^{\prime} q}^{c}+D E M_{h t q}^{c}-S_{h-1, t^{\prime} q}^{c} \\
& \forall h \in H, \forall t \in T, \forall q \mid q=1, \forall c \in S C
\end{aligned}
$$

In constraint (5-18) we observe the treatment of all patients that arrive in the $\mathrm{ED}\left(D E M_{h t q}^{c}\right)$. After this stage, the patients are distributed in accordance with the transfer rates.

First General Assessment Queue

$$
\begin{aligned}
& W_{h t q}^{c}=W_{h-1, t^{\prime}, q}^{c}+\alpha_{q} S_{h-1, t^{\prime}, q-1}^{c}-S_{h-1, t^{\prime}, q}^{c} \\
& \forall h \in H, \forall t \in T, \forall q \mid q=2, \forall c \in S C
\end{aligned}
$$

The constraint 5-19 establishes that patients waiting in each hour in the First General Assessment Queue are patients waiting previously in this stage $\left(w_{h-1, t, q}^{c}\right)$ adding patients from the triage phase $\left(\alpha_{q} s_{h-1, t, q-1}^{c}\right)$, and discounting the patiens served in the First General Assessment step $\left(s_{h-1, t, q}^{c}\right)$.

For the other phases of treatment, the number of patients waiting follow this same logic by changing only the parcels equivalent to the transfers of patients from other phases.

Medication Queue

$$
\begin{aligned}
& W_{h t q}^{c}=W_{h-1, t^{\prime} q}^{c}+\beta_{q} S_{h-1, t^{\prime}, q-3}^{c}+\eta_{q} S_{h-1, t^{\prime}, q+1}^{c}-s_{h-1, t^{\prime}, q}^{c} \\
& \forall h \in H, \forall t \in T, \forall q \mid q=5, \forall c \in S C
\end{aligned}
$$


Second General Assessment Queue

$$
\begin{aligned}
& W_{h t q}^{c}=W_{h-1, t^{\prime}, q}^{c}+\beta_{q} S_{h-2, t^{\prime \prime}, q-4}^{c}+\mu_{q} S_{h-2, t^{\prime \prime}, q-1}^{c}+\eta_{q} S_{h-2, t^{\prime \prime}, q}^{c}-S_{h-1, t^{\prime}, q}^{c} \\
& \forall h \in H, \forall t \in T, \forall q \mid q=6, \forall c \in S C
\end{aligned}
$$

Beds Queue

$$
\begin{aligned}
& W_{h t q}^{c}=W_{h-1, t^{\prime}, q}^{c}+\alpha_{q} S_{h-1, t^{\prime}, q-3}^{c}+\beta_{q} S_{h-1, t^{\prime}, q-2}^{c}+\alpha_{q-1} * \rho^{1} S_{h-1, t^{\prime \prime}, q-3}^{c} \\
& +\alpha_{q-1} * \rho^{2} * S_{h-1, t^{\prime \prime \prime}, q-3}^{c}+\eta_{q} S_{h-1, t^{\prime}, q+2}^{c}-S_{h-1, t^{\prime}, q}^{c} \\
& \forall h \in H, \forall t \in T, \forall q \mid q=4, \forall c \in S C
\end{aligned}
$$

Finally, constraints (5-23)-(5-27) define the domain of the decision variables.

$$
W_{h t q}^{c} \in \mathbb{R}_{+}, \quad \forall h \in H, \forall t \in T, \forall c \in S C
$$

$$
\begin{gathered}
x_{s d p} \in\{0,1\}, \quad \forall s \in S, \forall d \in D, \forall p \in P \mid \exists I \_ \text {PHYSICIAN } N_{s d p} \\
n P h y_{h t q} \in \mathbb{Z}_{+}, \quad \forall h \in H, \forall t \in T, \forall q \in Q \\
S_{h t q}^{c} \in \mathbb{R}_{+}, \quad \forall h \in H, \forall t \in T, \forall c \in S C \\
n N u r_{h t q} \in \mathbb{Z}_{+}, \quad \forall h \in H, \forall t \in T, \forall q \in Q
\end{gathered}
$$

\section{2}

\section{Simulation Modeling Framework}

Here the simulation also follows what is described in Section 3.2.4. The simulation model developed is based in Figure 5.2. Some assumptions take for the mathematical model are relaxed in the simulation model: (i) patients requiring vital care that are seen in the Life-Threatening room are not treated just as a delay but patients in this stage are attended by a physician that is assigned in the Beds room treatment (shared resources); (ii) all steps of treatment are no longer assumed to have a fixed duration but follow exponentially distributed interservice times; (iii) the system does not start empty. Instead we perform a warm-up period in the simulation model; (iv) the waiting time is estimated precisely since the simulation uses a discrete event paradigm. 


\section{3 \\ Numerical Experiments}

For numerical experiments, we present the application of the model presented in Section 5.1 to a real case study in a Brazilian hospital. The scenario generation was implemented in MATLAB R2013a. The mathematical programming model was implemented and solved using AIMMS 4.26 and GUROBI 7.5 with default settings. The solutions were achieved on an Intel i7 3.3 GHz 64GB RAM computer. The simulation model was implemented using Arena Simulation software version 14.7. All statistical analyses were performed using R software version 3.3.1.

The computational experiments using SAA were performed considering the Latin Hypercube Sampling (LHS) (see Chapter 3.1), since in Chapter 4 we see that this method perform better. We used the probability distribution (take from the fit test as specified in the Step 1 of the solution framework proposed in Section 3.2) to performed the random sample draws in the generation of scenarios regarding the arrival of patients (Demand). For the Step 2 of the solution framework proposed in Section 3.2 we define the parameters used in the SAA procedure defined in the Algorithm 1. For lower bound calculation, our in-sample analysis, we use 10 replications $(M=10)$ and we start using 20 scenarios $(N=20)$. We use an additional independent set of samples of 1,000 independent scenarios $(T=1$ and $\bar{N}=1,000)$, representing the true distribution, to calculate the upper bound, our out-of-sample analysis. If the stop criterion presented in the Section 3.2.2 is not achieved, the required number of scenarios $N$ is increased by 20. The stopping criterion for each replication was 3,600 s of computational time.

\section{4}

\section{Case study}

The case study addressed here was the same as the case study of Hospital B presented in the previous Chapter, in Section 4.6. The characteristics explained at that time remain the same, here we detail characteristics referring to the extent of the problem addressed in this chapter.

Here, we consider all patients of Clinical Medicine (CM) instead of just patients that go directly to the first assessment. After the triage, around $5 \%$ of patients go straight to the beds, and approximately $5 \%$ need vital care in the life-threatening room, and the others go to the first general assessment. Subsequently to the first general assessment, 33.68\% of the patients go to medication, $33.65 \%$ need auxiliary examinations, $10 \%$ go to the beds, and the others are discharged. Passing through medication, $25 \%$ of patients still 
need auxiliary examinations. After the second general assessment, 25\% of patients go to beds, $30 \%$ of patients need medication, and $15 \%$ of patients need other exams. From patients who need treatment in the life-threatening room, around $70 \%$ need auxiliary examinations. It is noteworthy that these transition percentages from one service phase to another are not stationary, i.e., variations may occur depending on the time and day of the week. However, from the analyzed data set, we cannot infer how this change happens, so we use the average transition rate in this study. The Figure 5.3 shows the transfer rate of patients from one treatment stage to another described above using the previously presented queuing system. 

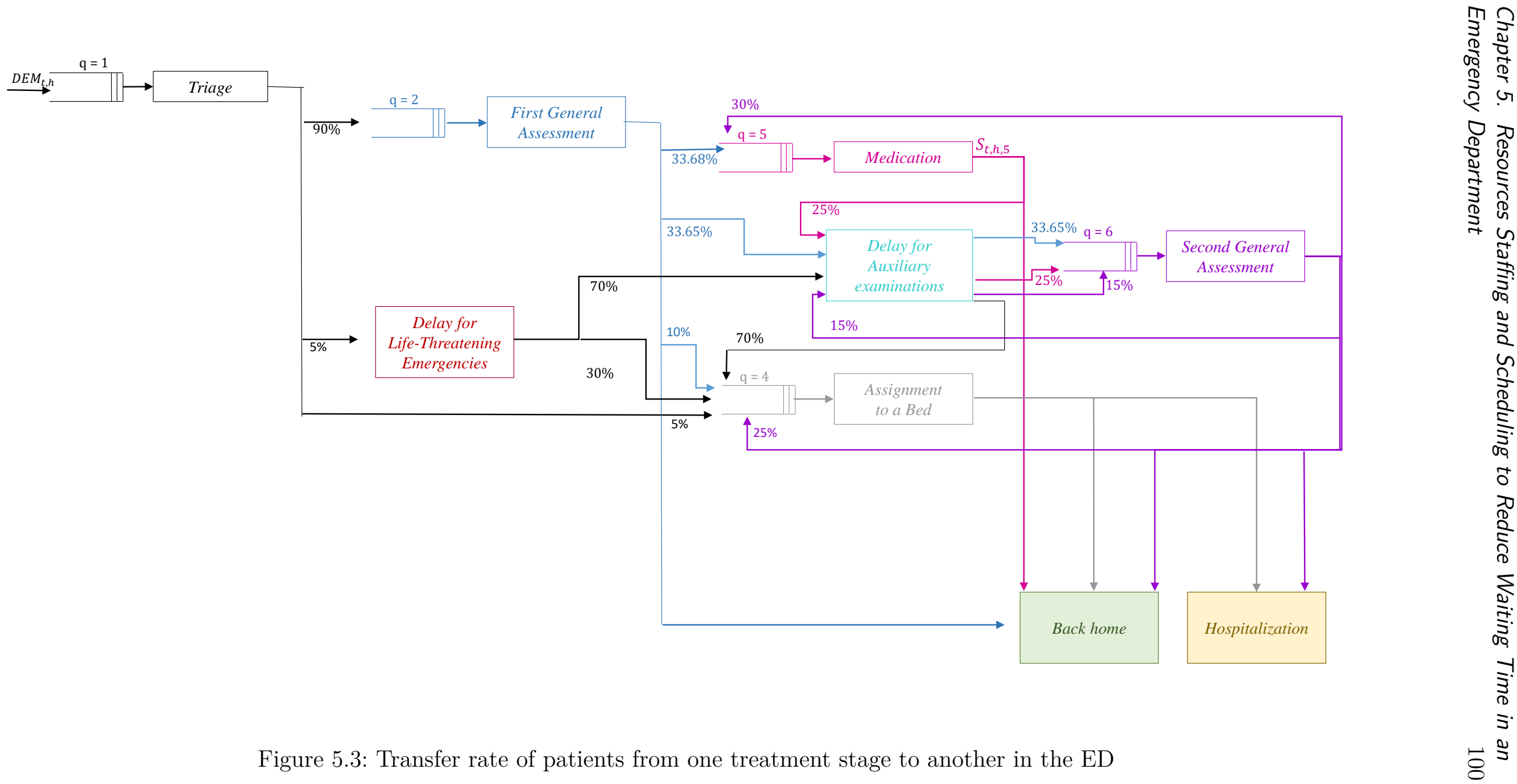

Figure 5.3: Transfer rate of patients from one treatment stage to another in the ED 
To determine service capacity, we adopted the assumption (corroborated by ED physicians and managers) that: each nurse in the triage can see 8 patients per hour, i.e., we considered the average service time to be 7.5 minutes; physicians in the first and second general assessment can see 3 patients per hour, i.e., we considered the average service time to be 20 minutes; physicians in the medication can treat 6 patients simultaneously; physicians in the beds can treat 4 patients simultaneously.

For numerical experiments in Chapter 4, as we were concerned just about the first general assessment, we determine a parameter $C A P \_M I N_{h t}$ which takes a value from 4 to 6 and guarantee a minimum number of physicians must always be available in the ED to cover the other treatment steps. Here, as we are staffing physicians to all phases of treatment, we remove this parameter.

\subsection{1}

\section{Simulation model}

For the simulation model of the studied ED, we assume the premises cited in section 5.2 and we consider an estimated service time as explained in the previous section, considering an average for the exponential distribution. We conducted a Kruskal-Wallis test which results indicated a statistically significant difference between patient interarrival times for each day of the week and hour (Kruskal-Wallis test, chi-squared $=8083.6$, $\mathrm{df}=23$, p-value $<2.2 \mathrm{e}-16)$. We used the Kruskal-Wallis test due to the non-normality of the data set (Shapiro test, $\mathrm{W}=0.7544$, $\mathrm{p}$-value $<2.2 \mathrm{e}-16$ ). Then, we perform a multiple comparison test (results are shown in Table L.1 of the Appendix L) to define which hours and days of the week could be considered to have the same arrival rate and, hence be grouped.

The current manually-defined ED schedule was used to define the warmup period and number of required replications, and to validate our simulation model. A warm-up period of 12 hours and a total of 100 replications were found to ensure that system occupancy corresponded to real starting conditions. Each replication simulated 6,585 hours of operations, which amounts to the total length of the period for which ED data was made available. Model validation was performed by comparing the real number of patients treated in the ED $(80,287$ patients) with the number of patients serviced in the simulation model $(80,051$ patients $)$, which yielded a variation of approximately $0.29 \%$ in the total number of treated patients. We considered such variation to be negligible and hence indicative of the simulated model's accuracy. 


\section{4 .2}

\section{Numerical results of the case study}

We start defining the number of scenarios that should be used in the experiments. We run preliminary tests as specified in Section 3.2.2 using Latin Hypercube Sampling (LHS). The size of the models (i.e.,variables and constraints), average execution times, and their variances are shown in Table M.1 of Appendix M. The average solution time of the experiments was 676.36 CPU seconds for 20 scenarios and 3,602.60s for 100 scenarios.

Table 5.5 present the results for each experiment in terms of the estimative of the optimality gap to the true solution (see Section 3.1.1.3), showing the confidence interval.

Table 5.5: Experiment results: estimative of the optimality gap and confidence interval

\begin{tabular}{cccc}
\hline \multirow{2}{*}{ Scenarios } & \%Gap & \multicolumn{2}{c}{ Confidence Interval } \\
\cline { 3 - 4 } & & Min & Max \\
\hline $\mathbf{2 0}$ & 5.163 & 4.717 & 5.609 \\
$\mathbf{4 0}$ & 2.440 & 2.144 & 2.736 \\
$\mathbf{6 0}$ & 2.000 & 1.722 & 2.279 \\
$\mathbf{8 0}$ & 1.004 & 0.828 & 1.179 \\
$\mathbf{1 0 0}$ & 0.014 & -0.042 & 0.069 \\
\hline
\end{tabular}

The gap values vary from $5.163 \%$ in the experiments with 20 scenarios to $0.014 \%$ in the experiments with 100 scenarios. Therefore, we notice that optimality gap achieves the stop criterion defined for 100 scenarios, being the $95 \%$ level of confidence interval (CI) 0.056; thus the gap considering the CI is lower than $1 \%$. So all results presented hereafter will be using this scenario setting.

To estimate the benefits of considering uncertainty into the model, we first use two indicators, which were presented by Birge and Louveaux [2011], the expected value of the perfect information (EVPI) and value of the stochastic solution (VSS).

The EVPI compares the performance of the solution from the recourse problem (solution here-and-now - RP), i.e., the solution of the stochastic model with recourse which here is the proposed model, when compared with a situation in which we have the perfect information, which means knowing the realization of the uncertainty (solution wait-and-see - WS). The EVPI is by definition, the difference between the RP and WS solutions:

$$
E V P I=|R P-W S|
$$


The VSS measures the gain in considering the uncertainty using the stochastic model rather than merely basing the decision on the averages. First, we consider the expected value problem, where all random variables are replaced by their expected values and we solve a deterministic program:

$$
E V=\min _{x \in X} z(x, \bar{\xi}),
$$

where $\bar{\xi}=\mathbb{E}(\xi)$, this program yields first stage solutions. Let $\bar{x}(\bar{\xi})$ be an optimal solution to (5-29), called the expected value solution and let EEV be the expected cost when using the solution $\bar{x}(\bar{\xi})$, thus a second optimization must then be performed after $\xi$ is realized fixing the optimal solution to (5-29). We have:

$$
E E V=\mathbb{E}_{\xi}(z(\bar{x}(\bar{\xi}), \xi))
$$

The VSS is then:

$$
V S S=|E E V-R P|
$$

The RP, WS, and EEV values are shown in Table 5.6.

Table 5.6: Values of the RP, WS and EEV

\begin{tabular}{cc}
\hline Measures & Value \\
\hline Proposed Model (RP) & 18.045 \\
WS & 8.01 \\
EEV & 100.974 \\
\hline
\end{tabular}

The values presented in the Table 5.6 represent the expected number of patients in the queue by the probability of each scenario considered. These values suggest the efficiency of the proposed formulation in incorporating the uncertainty. First, as the value of the WS solution is close to the RP solution we can consider that the stochastic model considered represents the uncertainties, i.e., represents the demand of the hospital since the WS solution is always taken when the scenerio is known. Second, as the value of the EEV is greater than RP, we can conclude that considering only the average demand to define the staffing can lead to a larger number of patients in the queue.

Table 5.7 shows the stochastic indicators calculated using the measures presented in the Table 5.6. 
Table 5.7: Values of indicators used to estimate the benefits of considering uncertainty in the model

\begin{tabular}{cc}
\hline Stochastic Indicators & Values \\
\hline EVPI & 10.035 \\
VSS & 82.928 \\
\hline
\end{tabular}

The value 10.035 of the EVPI represents the difference between the solution obtained by the decision maker with perfect prediction power (WS) and the decision maker that solves the problem under uncertainty (RP), that is, the lower the EVPI value, the better the stochastic model accommodates the uncertainties. The VSS of 82.928 can be interpreted as an expected gain of the decision maker who considers the uncertainty or as the expected loss of the decision maker that opted for deterministic modeling using the mean of the stochastic parameters. In this first analysis, the value of the VSS seems to justify stochastic modeling.

We also use the model to evaluate the current schedule, which means that we compare the real schedule used by the ED during the planning horizon addressed in this study and run the proposed model. Using the current schedule, the objective function of the model was 2,514.583, comparing with the results presented in Table 5.7 we note a significant improvement.

After this first analysis, we used the discrete-event simulation model to estimate the benefits of our proposed schedule (recommended ED schedule for the hospital from the optimization model) against the schedule currently in use at the ED. Results of the numerical experiments are presented in Table 5.8. The columns titled "Current Allocation" includes the value of each indicator associated with the current manually-defined ED schedule. In the columns titled "Proposed Model", we show results obtained through discrete-event simulation of the optimal schedule generated by our proposed model (the RP). When presenting results obtained by our optimization model, in addition to the value of each indicator, we also report the $95 \%$ level of confidence interval (CI). We present the results for each treatment phase, and we also show the overall average waiting time. 
Table 5.8: Discrete-event simulation results

\begin{tabular}{|c|c|c|c|c|c|}
\hline \multirow{2}{*}{ Treatment phase } & \multirow{2}{*}{ Indicators } & \multicolumn{2}{|c|}{ Current Allocation } & \multicolumn{2}{|c|}{ Proposed Model } \\
\hline & & Value & CI & Value & CI \\
\hline \multirow{6}{*}{ First General Assessment } & Frequency of queue (\%) & 31.14 & 0.00 & 12.16 & 0.00 \\
\hline & Queue above 10 patients (\%) & 11.02 & 0.00 & 1.40 & 0.00 \\
\hline & Queue between 1 and 10 (\%) & 20.12 & 0.00 & 10.76 & 0.00 \\
\hline & Average number in queue & 3.05 & 0.05 & 0.59 & 0.01 \\
\hline & Average waiting time (min) & 36.55 & 0.54 & 7.17 & 0.16 \\
\hline & Average scheduled utilization (\%) & 51.60 & 0.00 & 41.59 & 0.00 \\
\hline \multirow{6}{*}{ Medication } & Frequency of queue (\%) & 5.58 & 0.00 & 10.38 & 0.00 \\
\hline & Queue above 10 patients (\%) & 0.18 & 0.00 & 0.29 & 0.00 \\
\hline & Queue between 1 and $10(\%)$ & 5.40 & 0.00 & 10.10 & 0.00 \\
\hline & Average number in queue & 0.18 & 0.01 & 0.33 & 0.01 \\
\hline & Average waiting time (min) & 4.46 & 0.22 & 7.94 & 0.14 \\
\hline & Average scheduled utilization (\%) & 30.71 & 0.00 & 38.40 & 0.00 \\
\hline \multirow{6}{*}{ Second General Assessment } & Frequency of queue $(\%)$ & 45.48 & 0.00 & 21.09 & 0.00 \\
\hline & Queue above 10 patients (\%) & 6.71 & 0.00 & 0.99 & 0.00 \\
\hline & Queue between 1 and $10(\%)$ & 38.76 & 0.00 & 20.10 & 0.00 \\
\hline & Average number in queue & 2.52 & 0.02 & 0.74 & 0.01 \\
\hline & Average waiting time (min) & 55.74 & 0.50 & 16.52 & 0.24 \\
\hline & Average scheduled utilization (\%) & 65.35 & 0.00 & 47.12 & 0.00 \\
\hline \multirow{7}{*}{ Beds } & Frequency of queue (\%) & 1.74 & 0.00 & 5.08 & 0.00 \\
\hline & Queue above 10 patients (\%) & 0.00 & 0.00 & 0.01 & 0.00 \\
\hline & Queue between 1 and 10 (\%) & 1.74 & 0.00 & 5.06 & 0.00 \\
\hline & Average number in queue & 0.04 & 0.00 & 0.11 & 0.00 \\
\hline & Average waiting time (min) & 1.26 & 0.05 & 3.89 & 0.11 \\
\hline & Average scheduled utilization (\%) & 24.86 & 0.00 & 36.20 & 0.00 \\
\hline & Overall average waiting time (min) & 54,56 & 0.52 & 16.83 & 0.18 \\
\hline
\end{tabular}

The optimal schedule leads to a reduction of approximately $61 \%$ and $54 \%$ in the frequency of queues for 'First General Assessment' and 'Second General Assessment', respectively, when compared with the current empirically-defined schedule. Moreover, for 'First General Assessment', the frequency of queue with more than 10 patients is reduced by approximately $87 \%$. For 'Second General Assessment', this same indicator achieves a reduction of about $85 \%$. The proposed model leads to a reduction of approximately $81 \%$ and $71 \%$ in the number of patients in queue for 'First General Assessment' and 'Second General Assessment', respectively, while the average waiting time decreases by approximately $80 \%$ and $70 \%$ in relation to the current schedule, respectively for 'First General Assessment' and 'Second General Assessment'. Although we observe the reduction of all queue indicators for 'First General Assessment' and 'Second General Assessment' treatment phases, we observe an increase in the queue indicators for 'Medication' and 'Beds' treatment phases. As we do not increase the number of physicians used, the model distributes the doctors between the areas and schedules to reduce the total waiting time, and for this, some areas have their queue indicators impaired to the detriment of the others. As we can see the overall average waiting time is reduced from 55' to $17^{\prime}$ 'using 
the model when compared to the current schedule.

It is worthy of mentioning that we do not report the indicators about the 'Triage' because the staffing defined by the model was the same used in the current allocation, thus the queue indicators are similar.

Taken together, these results indicate that the schedule generated by the proposed model takes advantage of the wide representation of the uncertainty in the arrival of patients by the generation of scenarios and thus achieves better results than those from the current manually-defined ED schedule.

\section{5}

\section{Conclusions}

The methodology we propose can optimally generate a schedule that aligns staff service capacity with patient arrivals, while accounting for all schedule requirements and contractual agreements. Aligning physician schedule with patient demand in all stages of treatments is a strategy for improving patient throughput in the ED, reducing the average LOS. Furthermore, our methodology is generalizable to other EDs, i.e., we can extend the model to different ED configurations that present other queue rules, schedule requirements, and contractual agreements. The main limitation of our study deals with the fact that we assumed fixed and constant staff service rates across the entire planning horizon for each stage of treatment. Nevertheless, as shown in Kuo [2014], this assumption is acceptable in an ED setting because emergency physicians are cross-trained (i.e., equipped with all essential medical knowledge for potential illnesses and injuries of patients in EDs) and must work as efficiently as possible; moreover this assumption is relaxed in the simulation. 


\section{6 \\ Coordinating physician scheduling for clinic and surgery ap- pointments to deliver timely access in a specialized surgical unit}

In this chapter, we present a description of the problem of resource assignment in a specialty surgical unit to properly coordinate physician scheduling to clinic and surgery appointments. In the sequence, we present the mathematical model formulated to represent the problem. Finally, we present the results of a real case study in a hospital from the USA.

\section{1}

\section{Problem Description}

The problem in question can be defined as the physician scheduling in a surgical specialty division (SSD), where one seeks to determine the activities each physician should handle in each day of the planning horizon in accordance with service requirements and contractual agreements, and the distributions of demand through the physicians coordinating clinic and surgery appointment, taking into consideration uncertainty in demand. Such decisions arise in the context of tactical capacity planning of health care staffs. Specialty surgical practice faces a significant challenge in scheduling physicians to maintain a proper balance of clinic and surgery appointments. Time in clinic results in surgical procedures to be performed by the physician while time in the operation room (OR) reduce the number of patients waiting for surgery. Inappropriate time assigned both to the clinic and OR derive in the problem of delivering timely and convenient access to health services. This problem is motivated by the desire to properly coordinate physician scheduling to clinic and surgery appointments to minimize the number of patients waiting for treatment, and consequently the access time, in a highly specialized surgical unit. Many surgical divisions around the world are observing a raise in patient demand that surpasses the increase of capacity, leading to long indirect wait times. We define "indirect wait time" or "access delay" to appointment as the number of business days between the day a patient is referred to the specialty surgical unit and the day the appointment (both for clinic encouter and surgery) occurred (see Gupta and Denton [2008] about direct vs. indirect 
time). Bearing in mind that what connects demand and capacity is how the unit handles access to services, we propose three different prioritization policies: access to clinic, access to surgery (itinerary length) or total access.

Typically, patients referred to a SSD have surgery as a treatment option due to their medical condition. In general, the process begins when patients call the department requiring a clinical appointment. During the clinical appointment, the surgeon and the patient discuss treatment options, and once the surgery is feasible, the surgery is scheduled. The patient flow through the SSD is represented as a queuing system with the treatment steps and the resources. Figure 6.1 represents an example of the flow in the SSD. We have the patient flow, which is different each day. Patients referred to the surgical department wait in a queue ('virtual queue') until the clinical encounter be performed for one of the physicians. Then, if the surgery is required for the treatment (a percentage $\alpha$ of the patients served each day in the clinic $\left(S_{t}\right)$ needs surgery), the patient waits again until the day of the surgery, also in a 'virtual queue'. The surgery is mandatory to be performed by the same doctor who attends the patient in the clinical appointment. Here we are concerned about the indirect time since the patients are wait from the moment they require an appointment until de day of the consultation/surgery instead the wait time in the day of the procedure (direct time) and because of this we call 'virtual queue' since the patients are not waiting in a waiting room.

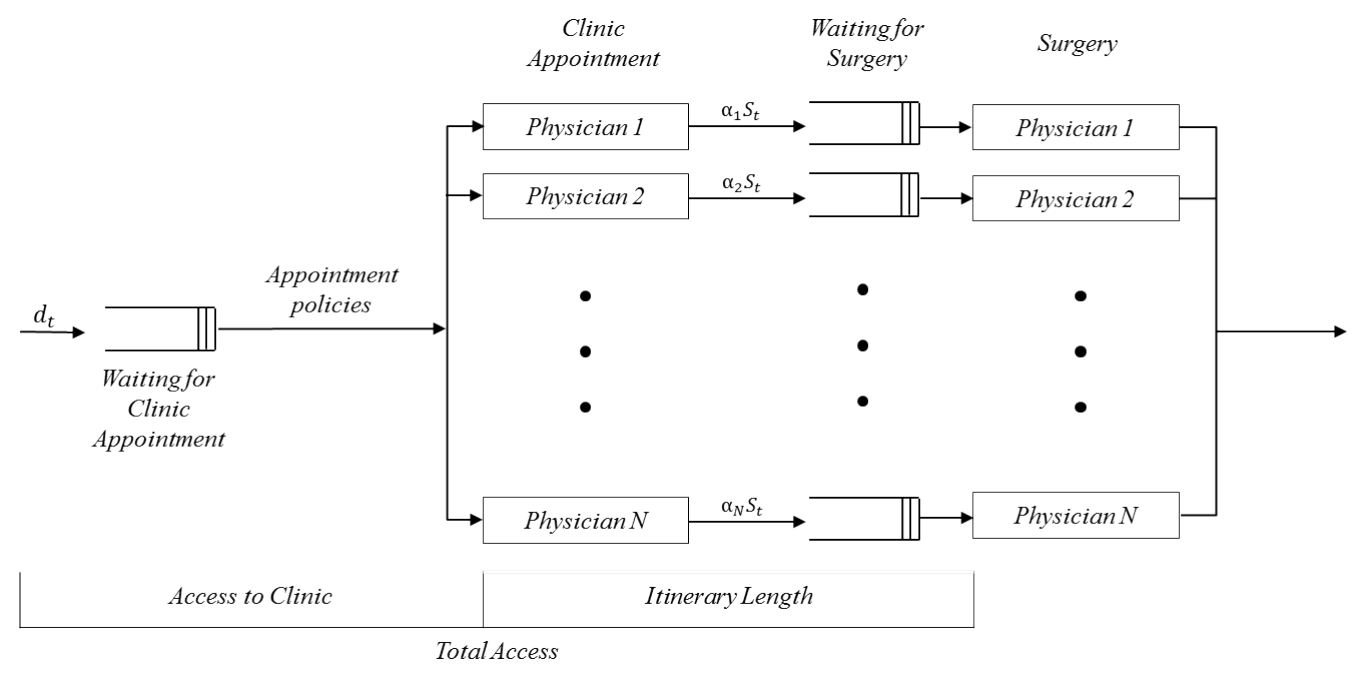

Figure 6.1: General patient flow in a surgical specialty division

According to how the surgical unit want/need to handle the access to services, we define three different prioritization policies: access to clinic, access to surgery (itinerary length) or total access. As we observe in Figure 6.1, when the objective is to achieve shorter waiting time for clinic appointments, 
the policy defined is the access to clinic; when the objective is to achieve shorter waiting time for the surgery, the policy defined is the access to surgery (also called itinerary length); and when the objective is a balance waiting time between clinic appointment and surgery the policy defined is the total access.

The problem is performed for a planning horizon that consists of a set of subsequent days of finite and fixed length $T$. We assume that a fixed number of physicians $P$ must be scheduled over the planning horizon through $K$ different assignment types. Figure 6.2 shows an example of physician scheduling in the SSD. For each day in the planning horizon, the physician should be assigned to the clinic $(\mathrm{C})$ or to the operation room (OR) in order to meet the demand, but the physicians should also be assign to other activities, e.g. Administrative, Research and Education (A/R/E) and Paid Time Off (D), in order to respect the service requirements and contractual agreements.

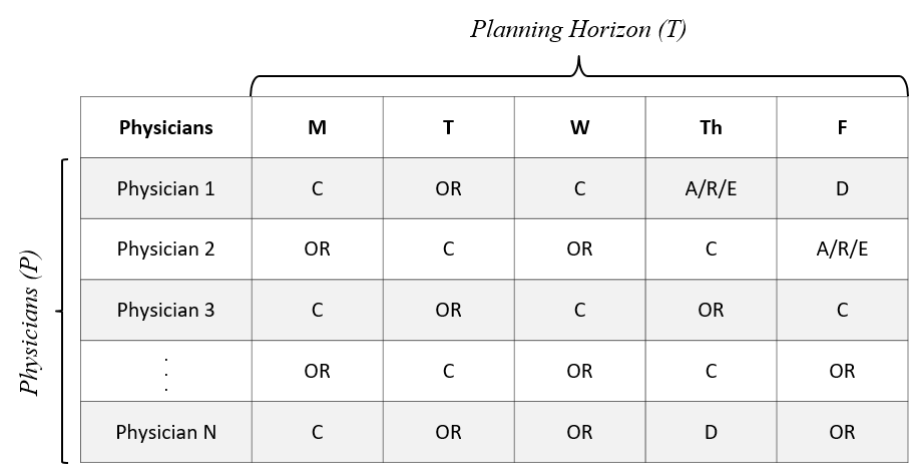

Figure 6.2: Example of physician scheduling in a surgical specialty division

To address this issue, we propose a two-stage stochastic modeling formulation. The mathematical model objective is to minimize the number of patients waiting for clinic and for surgery appointments that are weighted in the objective function by a factor $\beta$ and $1-\beta$, respectively, where $\beta \in[0,1]$ should vary according to the policy adopted by the surgical unit. Therefore, the model aims to guarantee the proper balance of physician assignments to clinic and surgery appointments to meet patient demand according to the policy adopted, while taking into account scheduling requirements and contractual agreement to perform the assignments to other activities that physicians should deal. The first-stage decisions define the physicians' assignments, i.e. define the activity each physician will perform in each day to meet the scheduling requirements and the contractual agreement; the second-stage decisions are the evaluation of queue level for clinic and surgery given the physician assignment for a certain realization $\xi$ of the uncertain parameters. The uncertainties in the model are related to the levels of demand (patients referred to a specialty surgical unit) 
for a clinic appointment and consequently for surgeries, which are modeled as random variables.

We found some related papers to this study: Gunawan and Lau [2010], Gunawan and Lau [2013], Baugh [2012], Martinez et al. [2016a], Martinez et al. [2016b], and Kazemian et al. [2017]. Martinez et al. [2016b] and Kazemian et al. [2017] address the scheduling of patients referred to a surgical specialty and study the impact of scheduling policies on clinical and surgical access using discrete-event simulation. The objective in both is to reduce waiting times to access elective surgery, coordinating clinical and surgical appointments. Martinez et al. [2016a] analyze strategies to assign surgeons in an elective surgical practice and to evaluate the clinical and surgical capacity by using a simulation model. Their objective is to identify inefficiencies and to point strategic changes to the schedule to achieve better allocation of surgeon resources. Baugh [2012] use a simulation model to investigate how access to care responds to surgeons' time allocation policies and other factors. Gunawan and Lau [2010] and Gunawan and Lau [2013] develop a model to assign physician duties to the defined time slots/shifts over a planning horizon to satisfy as many physicians' preferences and services requirements as possible while ensuring the optimal use of the resources. As duties, they refer to surgery, clinic appointments, scopes, calls, administration. There are some differences between these studies and ours. First, we consider the physician scheduling as opposed to considering patient scheduling policies once we aim to improve access to care better distributing the capacity of this scarce resource among the services. Second, we use an optimization model to automatically coordinate the assignment of the physicians to clinic, OR and other tasks by considering all service requirements, contractual agreement, and uncertainty in the demand; whereas Martinez et al. [2016a] and Baugh [2012] just evaluate the schedule that is manually done in the former and for a single physician in the latter. Third, our paper focuses on the access to care, the principal difference from Gunawan and Lau [2010] and Gunawan and Lau [2013], and for this, we incorporate the factor demand in the model on which we consider the associated uncertainty to better allocate the physicians resources over time and activities.

The most important difference between our work and the papers in literature related to scheduling problem in health care is that we extend our view to integrating the scheduling of clinic and surgery appointments such that the physician capacity is distributed through the days in clinic, ORs and other activities they may handle and the indirect waiting time of the patients for both the clinic and surgery appointment is minimized according 
to the policies proposed. This model also considers the continuity of care between patient-surgeon, once to assign the capacity we ensure the number of surgeries derived from consultations in the clinic is delivered by the same surgeon. The concept of coordinating physician scheduling to clinic and surgery appointments considering different prioritization policies and addressing the uncertainty in demand is unique and novel in our work and, to the best of our knowledge, has never been studied before in the context of none of the scheduling areas mentioned previously.

Table 6.1 summarizes the contributions of this chapter comparing with related works in literature. 
Table 6.1: Summary of contributions from the Chapter 6

\begin{tabular}{|c|c|c|}
\hline Chapter 6 & \multicolumn{2}{|l|}{ Literature } \\
\hline $\begin{array}{l}\text { Physician scheduling - integrate the scheduling of clinic } \\
\text { and surgery appointments }\end{array}$ & Patient scheduling policies & $\begin{array}{l}\text { e.g. [Kazemian et al., 2017; Martinez } \\
\text { et al., 2016b] }\end{array}$ \\
\hline $\begin{array}{l}\text { Optimization model - automatically coordinate the assign- } \\
\text { ment of the physicians }\end{array}$ & Simulation model - evaluate schedule manually done & e.g. [Martinez et al., 2016a] \\
\hline Multiple physicians & Just one physician & e.g. [Baugh, 2012] \\
\hline Focus on the access to care - consider demand uncertainty & $\begin{array}{l}\text { Focus just in perfom the scheduling of physicians over the } \\
\text { diferente tasks }\end{array}$ & e.g. [Gunawan and Lau, 2010, 2013] \\
\hline
\end{tabular}


We assign the physician to a task for the entire day, and the allocation in the OR and clinic is based on institutional standards; we assume the surgeon's block time is predetermined. Our model is concerned with choosing (1) the activity that should be assigned to the physician, (2) the number of clinic appointment performed in each day per physician, and (3) the number of surgeries performed in each day per physician.

\section{2}

\section{Mathematical Model Framework}

In this section we present the mathematical model developed to deal with the aforementioned problem. The objective of the mathematical model is to minimize the expected value of the number of patients waiting according to the policy adopted by the surgical unit, while taking into account scheduling requirements and contractual agreement for each physician. Table 6.2 presents the domains in which our model attributes are defined, while Tables 6.3 and 6.4 provide a complete listing of parameter and variable definitions.

Table 6.2: Sets, subsets and corresponding domains

\begin{tabular}{llll}
\hline Sets & Indexes & Domain & Description \\
\hline Physicians $(P)$ & $\mathrm{p}$ & $\{1, \ldots,|P|\}$ & Physicians of the surgical division \\
Days $(T)$ & $\mathrm{t}, \mathrm{t}$ " & $\{1, \ldots,|T|\}$ & Days in the planning horizon \\
Assignments Type $(K)$ & $\mathrm{k}$ & $\{0, \ldots,|K|\}$ & Places that the physician can be assigned $(\mathrm{OR}, \mathrm{Clinic} . .)$. \\
Scenarios $(\Xi)$ & $\xi$ & $\{0, \ldots,|\Xi|\}$ & Possible realization scenarios \\
Days other tasks $(\tau)$ & - & Subset of Days $(T)$ & Days where assignments to other tasks can be added \\
OR $(O)$ & - & Subset of Assignments Type $(K)$ & Assignments type related to tasks in OR \\
Clinic $(C)$ & - & Subset of Assignments Type $(K)$ & Assignments type related to tasks in Clinic \\
Clinic and OR $(C O)$ & - & Subset of Assignments Type $(K)$ & Assignments type related to tasks in Clinic and OR \\
\hline
\end{tabular}

Table 6.3: Model parameters

\begin{tabular}{lll}
\hline Parameters & Description & Unit \\
\hline$\beta$ & Weight assigned to objective function parcels & {$[0,1]$} \\
$n^{k}$ & Minimum number of assignments for type $k$ & - \\
$q$ & Maximum number of days the physician can be allocated consecutively in the OR & - \\
$u$ & Maximum number of ORs (nOR) available for the surgical division & - \\
$l$ & Minimum number of ORs that surgical division must use per day & - \\
$i_{p t}^{k}$ & Indication if physician $p$ in the day $t$ is allowed to be assigned to place $k$ & $\{0,1\}$ \\
$\kappa_{j t}^{k}$ & Capacity (number of appointments to be performed) for each physician $p$ in day $t$ for each assignment & - \\
& type $k$ & - \\
$\alpha$ & Demand (arrival of patients for each day $t$ ) in realization $\xi$ & - \\
\hline
\end{tabular}


Table 6.4: Model variables

\begin{tabular}{lll}
\hline Variables & Description & Domain \\
\hline$w_{t \xi}^{\text {cli }}$ & Number of patients wait for clinic appointment in day $t$ in realization $\xi$ & $\mathbb{R}_{+}$ \\
$w_{p t \xi}^{\text {sur }}$ & Number of patients wait for surgery in day $t$ for physician $p$ in realization $\xi$ & $\mathbb{R}_{+}$ \\
$x_{p t}^{k}$ & Decision if physician $p$ is allocated to place $k$ in the day $t$ & $\{0,1\} \mid \exists i_{p t}^{k}$ \\
$y_{p t \xi}^{k}$ & Number of patients assigned to physician $p$ in the day $t$ for surgery in realization $\xi$ in place & $\mathbb{Z}_{+}$ \\
$d_{p t \xi}^{\text {sur }}$ & $k$ & $\mathbb{Z}_{+}$ \\
\hline
\end{tabular}

The proposed mathematical formulation is as follow:

$$
\operatorname{Min} Z=\beta * \sum_{t \in T} w_{t \xi}^{\mathrm{cli}}+(1-\beta) * \sum_{p \in P} \sum_{t \in T} w_{p t \xi}^{\mathrm{sur}}
$$

The objective function in (6-1) consists of minimizing the total number of patients waiting for clinic and surgery appointments weighted in the objective function by a factor $\beta$ and $1-\beta$, respectively. The prioritization of one policy over another occurs in the definition of the $\beta$ factor.

Subject to:

$$
\sum_{k \in K} x_{p t}^{k}=1 \quad \forall p \in P, \forall t \in T
$$

Constraint (6-2) guarantees that each physician is assigned to just one place each day.

$$
\sum_{t \in \tau} x_{p t}^{k} \geq n^{k} \quad \forall p \in P, \forall k \in K
$$

Constraints (6-3) matches the minimum time the physician should have in each assignments type.

$$
\sum_{t^{\prime \prime} \geq t}^{t " \leq t+q} \sum_{k \in O} x_{p t}^{k}, \leq q \quad \forall p \in P, \forall t \in T
$$

Constraint (6-4) establishes that each physician can just be assigned in the OR for at most $q$ days consecutively.

$$
\begin{aligned}
& \sum_{p \in P} \sum_{k \in O} x_{p t}^{k} \leq u \quad \forall t \in T \\
& \sum_{p \in P} \sum_{k \in O} x_{p t}^{k} \geq l \quad \forall t \in T
\end{aligned}
$$

Constraints (6-5)-(6-6) enforces that the number of Physicians assigns to OR per day is not higher than the maximum number of ORs the department can use and not lower than the minimum number of ORs the department need to use, respectively.

$$
y_{p t \xi}^{k} \leq x_{p t}^{k} \kappa_{j t}^{k} \quad \forall p \in P, \forall t \in T, \forall k \in O C, \forall \xi \in \Xi
$$


Constraint (6-7) ensures that patients will only be assigned to an appointment to a physician in a certain place if the physician is allocated to that place in that day and has available capacity to perform the medical encounter.

$$
w_{t \xi}^{\mathrm{cli}}=w_{t-1, \xi}^{\mathrm{cli}}+d_{t-1, \xi}-\sum_{p \in P} y_{p t \xi}^{k} \quad \forall t \in T, \forall k \in C, \forall \xi \in \Xi
$$

Constraint (6-8) computes the number of patients waiting for a clinic appointment each day.

$$
\begin{gathered}
d_{p t \xi}^{\text {sur }} \leq \alpha y_{p, t-1, \xi}^{k} \quad \forall p \in P, \forall t \in T, \forall k \in C, \forall \xi \in \Xi \\
d_{p t \xi}^{\text {sur }} \geq \alpha y_{p, t-1, \xi}^{k}-1 \quad \forall p \in P, \forall t \in T, \forall k \in C, \forall \xi \in \Xi
\end{gathered}
$$

Constraint (6-9)-(6-10) establishes the surgery demand for each physician. Surgery demand is a rate of the clinic appointments performed by each physician.

$$
w_{p t \xi}^{\mathrm{sur}}=w_{p, t-1, \xi}^{\mathrm{sur}}+d_{p t \xi}^{\mathrm{sur}}-y_{p t \xi}^{k} \quad \forall p \in P, \forall t \in T, \forall k \in O, \forall \xi \in \Xi
$$

Constraint (6-11) computes the number of patients waiting for surgery each day for each physician.

\section{3 \\ Numerical Experiments}

In this section, we show the results of the computational experiments conducted with the proposed model, considering a real case study on the colorectal surgery (CRS) division. The two-stage stochastic programming model and the sampling technique were implemented using AIMMS version 4.46, and the MILP models were solved using GUROBI 7.5 with defaults settings. We performed the experiments in an Intel i5 $3.2 \mathrm{GHz}$ 8GB RAM computer.

To take into account the uncertainty in demand levels for patients referred to SSD, scenarios were generated by using the Poisson distribution being the lambda $(\lambda)$ the average number of patients referred SSD for each weekday, as following:

$$
d_{t \xi}=f\left(\lambda_{t}\right)
$$


The computational experiments using SAA were performed considering Monte Carlo Sampling (MCS) as scenario generation method. For this study we used Monte Carlo Sampling since convergence occurs for a small number of scenarios. For lower bound calculation, our in-sample analysis, we use 10 replications $(M=10)$ and we start using 5 scenarios $(N=5)$. We use an additional independent set of samples of 1,000 independent scenarios ( $T=1$ and $\bar{N}=1,000)$, representing the true distribution, to calculate the upper bound, our out-of-sample analysis. If the stop criterion presented in the Section 3.2.2 is not achieved the required number of scenarios $N$ is increased by 5 .

In the numerical experiments of this section the objective is to show how the number of patients in the queue changes as we vary the $\beta$ factor in the objective function and with this we constructed an efficiency frontier (number of patients waiting versus beta factor). Then, we discuss where each of the three prioritization policies defined are located on the efficiency frontier.

\subsection{1}

\section{Case Study}

The proposed model was tested in a real case study on the colorectal surgery (CRS) division of Mayo Clinic in Rochester, Minnesota. However, our general modeling framework can be used for other surgical units and other medical centers that offer clinic consultations followed by surgery. In the studied surgical division, physician scheduling is made for three months, but the planning is done two months in advance. Figure 6.3 shows an example of how we consider the planning horizon for the unit studied.

\begin{tabular}{|c|c|c|c|c|c|c|}
\hline \multirow{2}{*}{$\begin{array}{l}\text { Planning } \\
\text { Today }\end{array}$} & Fixed allocation & Fixed allocation & $O R \rightarrow 2 O R$ & Fixed allocation & Free assignment & \multirow{2}{*}{$\begin{array}{l}\text { Out of scope } \\
\text { Month } 6\end{array}$} \\
\hline & Month 1 & Month 2 & Month 3 & Month 4 & Month 5 & \\
\hline \multirow{2}{*}{$\begin{array}{c}\text { Planning } \\
\text { in the next } \\
\text { month }\end{array}$} & $\begin{array}{l}\text { Fixed allocation } \\
\text { (out of scope) }\end{array}$ & Fixed allocation & Fixed allocation & $O R \rightarrow 2 O R$ & Fixed allocation & Free assignment \\
\hline & Month 1 & Month 2 & Month 3 & Month 4 & Month 5 & Month 6 \\
\hline
\end{tabular}

Figure 6.3: Example of the planning horizon considered in the case study

From the Figure 6.3 what we observe is: the planning horizon considered is five months; in the Month 3 just small capacity adjustments is allowed which derives from the policy of CRS division in which physicians can use two operation rooms to perform more surgeries in one day. The second OR is assigned to a physician that is already allocated to perform surgery in a given day and preferably for physicians with more patients waiting for surgeries, thus these doctors have 2 ORs (called 2ORs activity) to that day; in the Month 5 
the physicians can be allocated to any activity according to the availability of each one $\left(i_{p t}^{k}\right)$; for the other months, a fixed allocation is used since there are several capacity slots already occupied with clinic appointments and surgeries based on previous physician scheduling, same reason why for Month 3 just small adjustment of capacity is allowed. Hence, the planning horizon has 100 days since we just consider weekdays. However, we included 10 more days in these experiments to better see the behavior of the model so the planning horizon analyzed here have 110 days $(|T|=110)$.

The CRS division under study consists of ten physicians $(|P|=10)$. Physicians can be assigned for 7 types of activities $(|K|=7)$ : operation room (OR), 2 operations rooms (2 ORs), clinic (C), small surgeries ( $G$ ), emergency room (ER), Administrative, Research and Education (A/R/E), and time not assigned in the unit (UN). The allocation for OR, 2OR and $\mathrm{C}$ is based on demand $\left(d_{t \xi}\right)$ that has different average number of patients referred to CRS division for each weekday $\left(\lambda_{t}=10.04,16.96,12.07,15.68,7.92\right)$. The allocation for other activities varies from physician to physician according to services requirements and contractual agreements in which a minimum assignment in each activity should be respected $\left(n^{k}\right)$. The physicians can be assigned for a maximum of 3 consecutive days in an OR $(q=3)$. Each physician can perform up to 8 encounters per day in the clinic and up to 3 surgeries per day in OR $\left(\kappa_{j t}^{k}\right)$. In this surgical division, an average of $68 \%$ of the patients seen in the clinic need surgery $(\alpha=0.68)$. The CRS division can use per day a maximum of 4 ORs $(u=4)$ and must use a minimum of 1 OR per day $(l=1)$.

An important assumption that is implied in traditional methods for queue studies is that the system is operating in a steady-state manner, in a sense that the initialization of variables should not be a concern. However, since we are proposing an optimization-based approach, it is mandatory to define initial queue levels. Therefore, we use a result of a preliminary simulation study (see Martinez et al. [2016a] and Martinez et al. [2016b] to details about the simulation) using the current configuration of CRS to define the initial queue for clinic appointments and surgery. Hence, the initial queue for clinic appointment is equal to 13 and for surgery equal to 168, being the queue for each physician as presented in Table 6.5.

Table 6.5: Initial queue for surgery per physician

\begin{tabular}{lrrrrrrrrrr}
\hline Physician & 1 & 2 & 3 & 4 & 5 & 6 & 7 & 8 & 9 & 10 \\
\hline Initial queue & 21 & 20 & 15 & 14 & 20 & 23 & 18 & 10 & 16 & 11 \\
\hline
\end{tabular}




\subsection{2 \\ Results}

We start defining the number of scenarios that should be used in the experiments. For this, we used the model considering the factor $\beta=0.5$ to run preliminary tests as specified in Section 3.2.2 using Monte Carlo Sampling (MCS) using. The optimal solution for each number of scenarios until achieve the stop criterion is shown in Table 6.6.

Table 6.6: Gap, lower and upper bound of SAA experiments

\begin{tabular}{ccccc}
\hline $\mathbf{N}^{\mathbf{a}}$ & Measures & $\mathbf{L B}^{\mathbf{b}}$ & $\mathbf{U B}^{\mathbf{c}}$ & gap \\
\hline \multirow{4}{*}{5} & Average & 2486.62 & 2796.01 & $309.39(12.44 \%)$ \\
& Deviation & 29.52 & 50.25 & 58.27 \\
& Deviation(\%) & 1.19 & 1.80 & 2.34 \\
\hline \multirow{4}{*}{10} & Average & 2506.62 & 2636.01 & $129.39(5.16 \%)$ \\
& Deviation & 25.51 & 81.29 & 85.19 \\
& Deviation(\%) & 1.02 & 3.08 & 3.40 \\
\multirow{4}{*}{15} & Average & 2577.73 & 2587.48 & $9.75(0.38 \%)$ \\
& Deviation & 18.82 & 7.87 & 20.39 \\
& Deviation(\%) & 0.73 & 0.30 & 0.79 \\
\hline
\end{tabular}

${ }^{a} \mathrm{~N}$ - Number of Scenarios

${ }^{\mathrm{b}} \mathrm{LB}$ - Lower Bound

${ }^{\mathrm{c}} \mathrm{UB}$ - Upper Bound

The results suggest that the variability is due to the number of scenarios considered to obtain the solution and tend to reduce as we consider more scenarios. This effect is related with the fact that, in general, a larger number of scenarios leads to a more comprehensive staffing and scheduling profile in terms of its ability to handle with higher demands, which makes the system more robust with respect to variations in the demand and consecutively achieves small fluctuations in second-stage. We notice that the optimality gap achieve the stop criterion defined for 15 scenarios, being the gap $0.38 \%$. Table 6.7 shows the minimum and maximum confidence levels for the optimal gap for each number of scenarios, which confirm that 15 scenarios can be considered sufficient to determine the lower and upper bounds of the objective functions since as for a confidence level of $95 \%$, the maximum value of the gap is $\leq 1 \%$. The size of the model (i.e., variables and constraints) and the average execution times are shown in Table N.1 in Appendix N. 
Table 6.7: Confidence interval of the optimal gap

\begin{tabular}{|c|c|c|}
\hline $\mathbf{N}^{\mathrm{a}}$ & \multicolumn{2}{|c|}{ 95\% Confidence leve } \\
\hline \multirow{2}{*}{5} & Min & 10.99 \\
\hline & Max & 13.89 \\
\hline \multirow{2}{*}{10} & Min & 3.06 \\
\hline & Max & 7.27 \\
\hline \multirow{2}{*}{15} & Min & -0.11 \\
\hline & Max & 0.87 \\
\hline
\end{tabular}

${ }^{a} \mathrm{~N}$ - Number of Scenarios

To construct the efficiency frontier we vary the value of the $\beta$ factor from 0 to 1 in the objective function (6-1) and we analyzed the results in terms of the number of patients waiting for clinic appointments, for surgery and the total number of patients waiting. Figures 6.4 shows the efficiency frontiers in terms of $\beta$ factor vs. the average number of patients waiting.

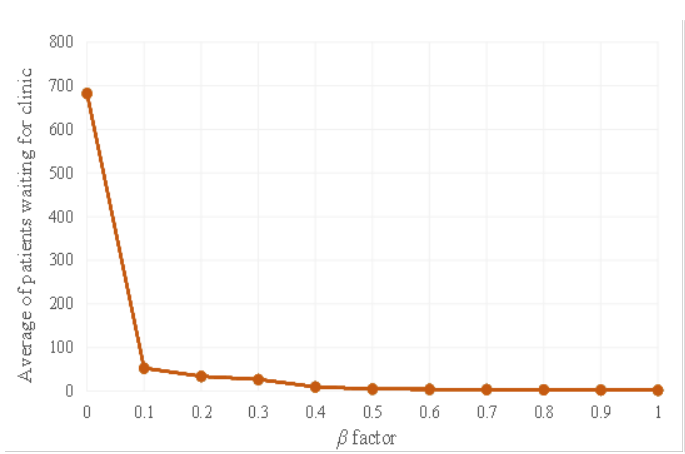

(a)

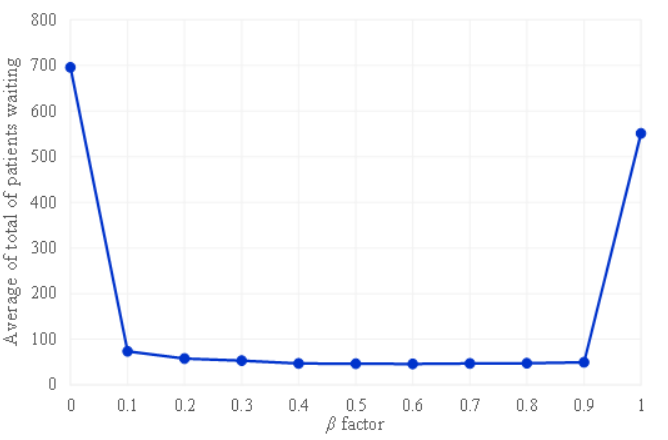

(c)

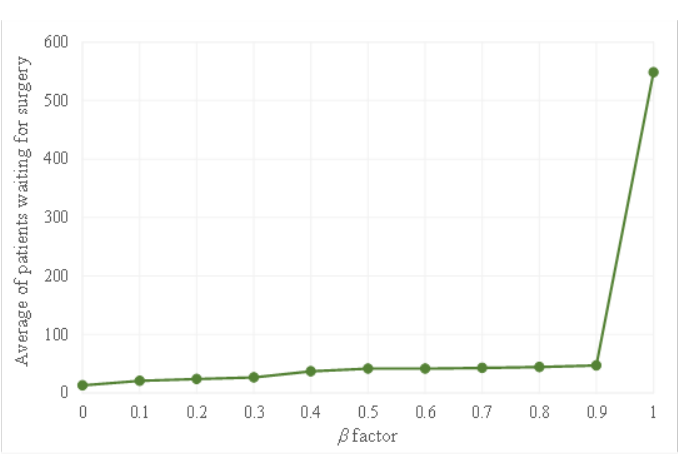

(b)

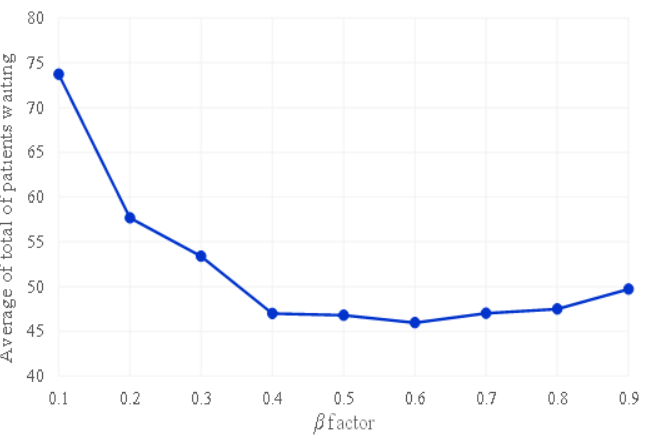

(d)

Figure 6.4: $\beta$ factor vs. average number of patients waiting: (a) for clinic appotinments; (b) for surgery; (c) total of patients waiting; (d) total number of patients waiting without extreme values of $\beta 0$ and 1

As can be seen, the efficiency frontier related to the total of patients 
waiting (Figure $6.4(\mathrm{c})$ ) is the combination of the graphs in Figure 6.4 (a) and (b) since the total number of patients waiting is the sum of the patients waiting in the two stages of the treatment. In the Figure 6.4 (d), we remove the extreme points ( $\beta$ factor $=0$ and $\beta$ factor $=1$ ) to better see the behavior of the midpoints in the curve. For Figure 6.4 (d), we note that the lowest values of the total number of patients waiting are achieved to $\beta$ between 0.4 and 0.6 being the minimum value reached using $\beta=0.6$. We summarize the results in terms of the average queue, worst case, and average idle capacity for clinic and surgery service for each value of $\beta$ factor in Table O.1 in Appendix O.

Figure 6.5 shows the efficiency frontier in terms of the average number of patients waiting for clinic appointments and the average number of patients waiting for surcerv.

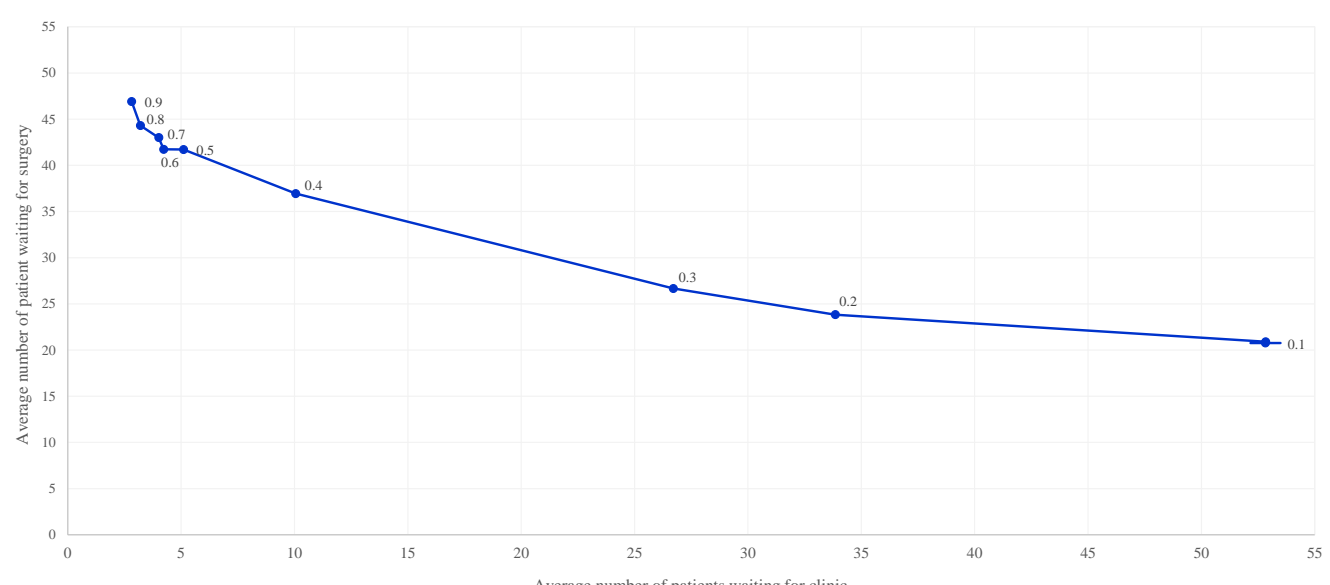

Figure 6.5: Average number of patients waiting for clinic appotinments vs. Average number of patients waiting for surgery

Each point on the graph showed in Figure 6.5 represents the result in terms of number of patients waiting for each $\beta$ chosen. The extreme points were removed since for $\beta=1$ we have only patients waiting for surgery and for $\beta=0$ we have only patients waiting for surgery, with no trade-off between the two measures.

Figures 6.6 (a) and (b) shows, respectively, the number of patients waiting for clinic appointment and surgery over the days in last month of the planning horizon using the $\beta$ factor $=0.6$ that is the value of $\beta$ which the total number of patients waiting is the lowest. In this Figure, we also plot the result of the observed schedule, which means that we fixed the real schedule used by CRS during the planning horizon addressed in this study and run the proposed model. 


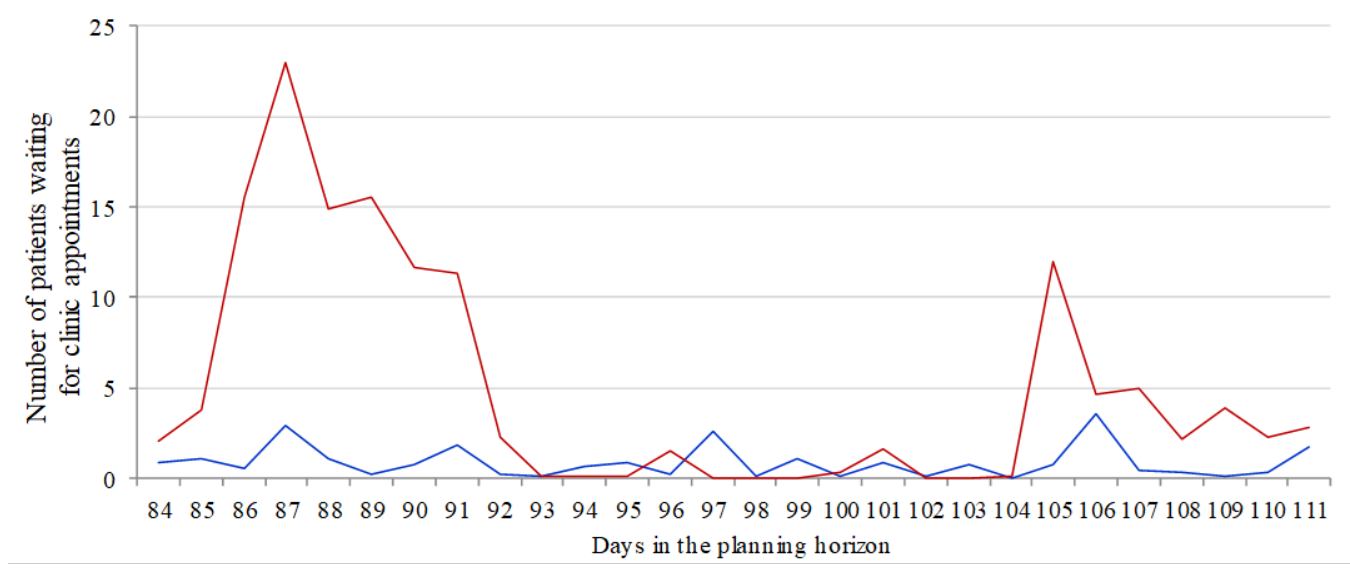

(a)

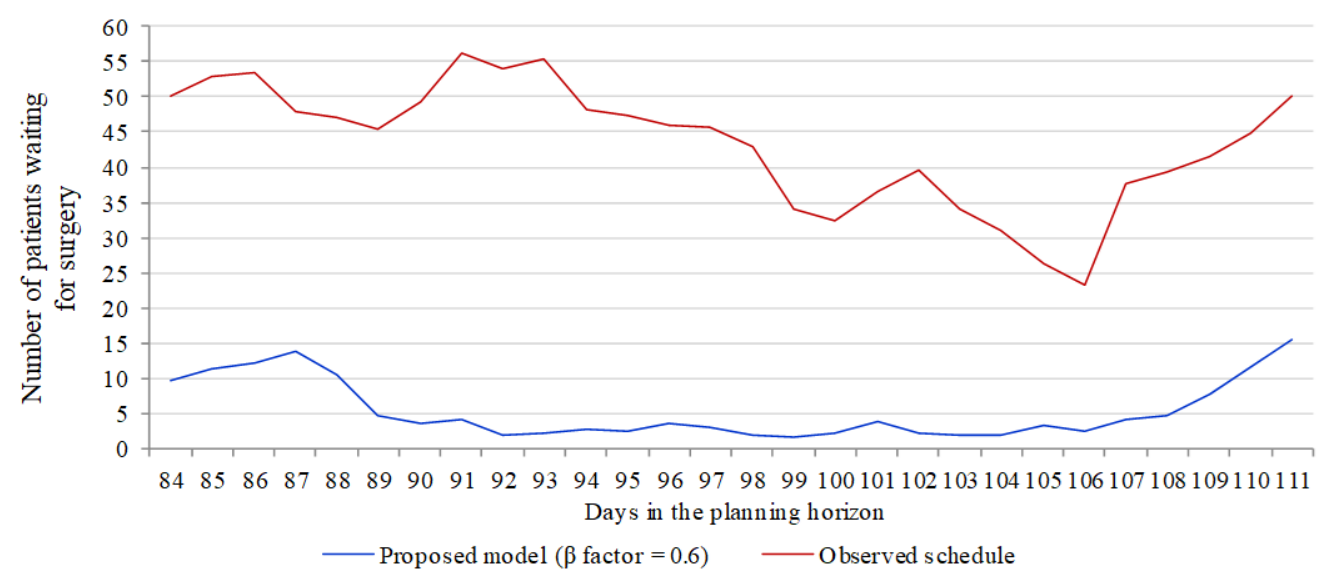

(b)

Figure 6.6: Comparation between the average number of patients waiting using the proposed model with $\beta$ factor $=0.6$ and the observed schedule (a) for clinic appointments; (b) for surgery

From Figure 6.6, we observe that using the proposed model it is possible to achieve better results in terms of the number of patients waiting than the schedule manually-defined by CRS since the curve of the 'Observed schedule' graph is mostly above the curve of the results of the proposed model. The schedule from the model using $\beta=0.6$ achieves a reduction of $82 \%$ and $98 \%$ in terms of the average number of patients waiting in the last month for clinic appointments and for surgery, respectively.

\subsection{3}

\section{Discussion}

This work presents a mathematical model to minimize the number of patients waiting for clinic and surgery according to the prioritization policies we propose: total access, access to clinic or access to surgery (itinerary length). 
The prioritization of one policy over another occurs in the definition of the $\beta$ factor. When setting a high value for $\beta$, we are working with the Access to Clinic policy, setting a low value for $\beta$ the policy Itinerary Length is defined, and settling intermediates values for $\beta$ the established policy would be the Total Access. We present a case of the colorectal surgery (CRS) division of a large academic medical center to test the model proposed.

From the results, we note that when we work with extreme values of $\beta$ ( $\beta$ $=0$ or $\beta=1$ ) the results are not good in terms of the total of patients waiting. When we set $\beta=1$ we are working with the policy Access to Clinic, so the prioritization is to patients waiting for clinic appointments and the model do not have the motivation to reduce the number of patients waiting for surgery thus the number of patients waiting in this stage of treatment is very high, contributing to increasing the number of total patients waiting. When we set $\beta$ $=0$, we are working with the policy Itinerary Length and occur the opposite. In this case, as the objective is to minimize just the number of patients waiting for surgery, which is the second stage of the treatment, the model decides not to flow the patients through the system, i.e., seeing patients in clinic and sending them to surgery, so the queue in surgery remains zero all the time while the queue in the clinic increase over the time. Also, from the results, we can say that setting $\beta$ from 0.4 to 0.8 we are working with the Total Access policy and we achieved very similar results using these values, being the better results in terms of the total number of patients waiting using $\beta=0.6$.

According to the patient's characteristics referred to the CRS, division studied and to the practice discussion with physicians, the values of $\beta$ indicated for this case study is the values related the Total Access policy. Patients referred to CRS division addressed in this work are mostly patients with some suspicion of cancer who need timely access both to clinic appointment with a specialist and to surgery, if surgery was the treatment indicated (which in that unit occurs on average $68 \%$ of the time). Comparing the results of the observed schedule with the results from the model using $\beta=0.6$, we see that using the schedule from the proposed model, the goal of balancing timely access between clinic and surgery is reached with more success. With the schedule used by CRS during the planning horizon addressed the number of patients waiting for surgery increase fast.

The model variation related to clinic access (high $\beta$ values) is suggested for practices where the patients have more urgency in the encounter with the specialist and are not worried about the time for surgery. For example, we can cite the plastic surgery unit where this situation can occur more frequently. In plastic surgery division, patients often want quick access to the expert for 
opinion and recommendation, but can wait longer for surgery. One reason is that these patients, in some cases, do not have sure that they want to perform the procedure.

For practices where the patients have more urgency in perform the surgery after pass through the clinic encounter and are not worried about the waiting time for clinic appointments, the suggestion is the model variation related to itinerary length (low $\beta$ values). For example, medical check-up units and other practices where the number of patients who need surgery is very low (the alpha is low), but when there are surgical cases, they require urgency.

\subsection{4}

\section{Conclusions}

In this chapter, we presented a novel framework for coordinating physician scheduling to clinic and surgery appointments in a highly-specialized surgical unit to minimize the number of patients waiting for treatment, and consequently, the access time. Proof of concept was given for the colorectal surgery (CRS) division of Mayo Clinic in Rochester, Minnesota. Knowing that what connects demand and capacity is how the unit deals with access to services, we proposed three different prioritization policies addressed by a mathematical model in which the prioritization of one policy over another occurs in the definition of a $\beta$ factor in the objective function. The model is based on the two-stage stochastic model, and we created possible realization scenarios to consider uncertainty in demand (patients referred to surgical specialty division) using Sample Average Approximation (SAA). Numerical results demonstrated that the model performed as expected with the variation of $\beta$ factor and can outperform the observed schedule from CRS. There is no single policy overall; the best policy depends on the goal, the case mix, and the wishes of the patients of each surgical division. For CRS, we consider the total access policy the most indicated; in this case, we consider total access when $\beta=$ 0.6. Total access performs $82 \%$ and $98 \%$ better than the observed schedule in terms of the average number of patients waiting in the last month for clinic appointment and for surgery, respectively. 


\section{Conclusions}

Healthcare providers face a significant challenge to meet demand properly since the demand for health services over the last few decades have raised due to the increased attention about preventive care and the aging population. Thus, healthcare administrators often effort to use existing capacity more efficiently. In this context, the healthcare staff scheduling is one of the modern healthcare challenges once inadequate scheduling leads to inappropriate patient treatment, decreasing both patient and provider satisfaction. In this thesis, we presented a framework to solve a set of problems related to healthcare staff scheduling. The models proposed are based on the two-stage stochastic programming, and we create possible realization scenarios to consider uncertainty in demand using Sample Average Approximation (SAA). To evaluate the models, we used real historical patient data from two hospitals in Brazil and one in the USA.

Concerning the first problem presented, we proposed a generalizable and integrated two-satge stochastic programming that, combined with a discreteevent simulation model, supports physician staffing and scheduling in the Emergency Department (ED) considering uncertainties related to patient arrivals able to reduce the time door-to-doctor. We validated and tested our model using real data from two EDs of Brazilian hospital, and the model proved to be adaptable to the two different realities. About the second problem, we proposed a mathematical model approach to provide timely access to the overall treatment in an Emergency Department. We proposed a twostage stochastic programming formulation which the aim is to guarantee that enough staff (physicians and nurses) will be available to meet patient demand to minimize the total number of patients waiting per hour. Moreover, the assignment of individual physicians is performed for each day and shift following service requirements and contractual agreements, taking into consideration uncertainty in patient arrival patterns. We validated and tested our model using real data from an ED of Brazilian hospitals. For both problems, our main contributions are the development a model that simultaneously supports physician staffing and scheduling, adequately accounts for service requirements, contractual agreements and the relation between installed capability and demand, 
all the while considering multiple overlapped shifts, different physician categories, and physicians preferences; the incorporation of uncertainties in patient arrivals by means of a two-stage stochastic programming using Sample Average Approximation; and, to validate and test our model, the application of the model in case studies using real data from Brazilian hospital EDs.

For the first problem, the proposed methodology enhances alignment between service capacity and demand, significantly improving all queue and waiting time indicators, which were evaluated using a discrete-event simulation model. In fact, for the hospital of case study I, the frequency of queue and average time door-to-doctor were reduced by $73 \%$ and $92 \%$, respectively, compared to the current manually-defined ED schedule, and for the hospital of case study II, the frequency of queue decrease about $22 \%$ and the average time door-to-doctor decrease 48\%. The Latin Hypercube Sampling performed better to this problem converging faster to solutions that are close to the real optimal solution of the problem comparing to Monte Carlo Sampling. For the second problem, the proposed methodology shows that aligning physician schedule with patient demand in all stages of treatments is a strategy for improving patient throughput in the ED, reducing the overall average LOS, which decrease $23 \%$ compared to the current manually-defined ED schedule. Both in the first and second problem addressed, our methodology facilitates and speeds up the generation of physician schedules in the EDs studied and ensures that all scheduling rules are met. It is also important to highlight that the proposed optimization approach is generalizable to other ED settings, with only a few minor adaptations.

In the last problem, we presented a novel framework for coordinating physician scheduling to clinic and surgery appointments in a highly-specialized surgical unit to minimize the number of patients waiting for treatment, and consequently the access time, in a highly specialized surgical unit. We proposed three different prioritization policies, and we discussed each of the three prioritization policies using an efficiency frontier. Proof of concept was given for the colorectal surgery (CRS) division of Mayo Clinic in Rochester, Minnesota. Our main contributions are the development a model that integrates the scheduling of clinic and surgery appointments, considers the continuity of care between patient-surgeon, presents the concept of different prioritization policies, all the while considering the uncertainty in demand. Numerical results demonstrated that the model can outperform the observed schedule from CRS. There is no single policy overall; the best policy depends on the goal, the case mix, and the wishes of the patients of each surgical division. For CRS, we consider the total access policy the most indicated, which performs $82 \%$ and 
$98 \%$ better than the observed schedule in terms of the average number of patients waiting in the last month for a clinic appointment and for surgery, respectively.

We consider that we accomplished with this thesis the objective of reducing the distance between theoretical research and real-world applications since we have successfully applied optimization knowledge to design decision support tools that ably solve the problems addressed.

\section{1}

\section{Future Perspectives}

For future work, the following points would be essential to research:

- Study the impact of service time when it is considered as a random variable in the optimization models, instead of just varying this parameter in the simulation;

- Perform a stress test for the proposed models, increasing the demand, decreasing capacity in the three models and increasing the number of phases of treatment in the second problem, in order to know the performance of the models in these conditions;

- Consider other staff categories in the model proposed in Chapter 5, considering two or more staff categories to the same stage of treatment. This characteristic increases the resolution complexity of the model.

- Generalize the model proposed in Chapter 5 to reflect the operational characteristics of several EDs without making any adaptation in the model, i.e., being only necessary to provide a different data set.

- Apply acceleration techniques (e.g Benders' Decomposition) to achieve solutions with lower computational time to enable future practical applications.

- Apply other solutions approaches manly in the problem of Chapter 5 as: Dynamic Programming and Multistage Optmization, Risk Aversion Control on the optmization, Distributionally Robust Optimization, and etc.

- Consider the effect of seasonality on patient demand and criticality (case $\operatorname{mix})$.

- Integrate the analysis of the areas studied (ED, surgical unit) with other areas of the hospital, for example, bed management, image center. The ED and the surgical unit are directly affected by the bed management, and the image center strongly impacts the ED, so the importance of the integrated analysis of these areas for a global solution. 
- Implement the proposed solutions in the hospitals studied to compare the current scenario with the improvements achieved in practice.

- Apply the proposed models in other hospitals. 


\section{Bibliography}

Bergh, J., Beliën, J., De Bruecker, P., Demeulemeester, E., and De Boeck, L. (2013). Personnel scheduling: A literature review. European Journal of Operational Research, 226(3):367-385.

Alfares, H. K. (2004). Survey, Categorization, and Comparison of Recent Tour Scheduling Literature. Annals of Operations Research, 127(1-4):145-175.

Bagheri, M., Gholinejad Devin, A., and Izanloo, A. (2016). An application of stochastic programming method for nurse scheduling problem in real word hospital. Computers \& Industrial Engineering, 96:192-200.

Bagwandeen, H. G. and Carroll, A. K. (2013). Systems and methods for automated triage and scheduling in an emergency department. US Patent App. 13/194,495.

Baker, K. R. (1976). Workforce Allocation in Cyclical Scheduling Problems: A Survey. Journal of the Operational Research Society, 27(1):155-167.

Bard, J. F., Shu, Z., and Leykum, L. (2013). Monthly clinic assignments for internal medicine housestaff. IIE Transactions on Healthcare Systems Engineering, 3(4):207-239.

Bard, J. F., Shu, Z., and Leykum, L. (2014). A network-based approach for monthly scheduling of residents in primary care clinics. Operations Research for Health Care, 3(4):200-214.

Bard, J. F., Shu, Z., Morrice, D. J., Leykum, L. K., and Poursani, R. (2016). Annual block scheduling for family medicine residency programs with continuity clinic considerations. IIE Transactions, 48(9):797-811.

Barz, C. and Rajaram, K. (2015). Elective Patient Admission and Scheduling under Multiple Resource Constraints. Production and Operations Management, 24(12):1907-1930.

Baugh, R. (2012). The impact of scheduling policies on surgical clinical access. The Journal of Medical Practice Management: MPM, 27(6):371.

Baum, R., Bertsimas, D., and Kallus, N. (2014). Scheduling, Revenue Management, and Fairness in an Academic-Hospital Radiology Division. Academic Radiology, 21(10):1322-1330. 
Bayraksan, G. and Morton, D. P. (2006). Assessing solution quality in stochastic programs. Mathematical Programming, 108(2-3):495-514.

Beaulieu, H., Ferland, J. A., Gendron, B., and Michelon, P. (2000). A mathematical programming approach for scheduling physicians in the emergency room. Health Care Management Science, 3(3):193-200.

Bechtold, S. E., Brusco, M. J., and Showalter, M. J. (1991). A Comparative Evaluation of Labor Tour Scheduling Methods. Decision Sciences, 22(4):683699.

Birge, J. R. and Louveaux, F. (2011). Introduction to stochastic programming. Springer Science \& Business Media.

Blöchliger, I. (2004). Modeling staff scheduling problems. A tutorial. European Journal of Operational Research, 158(3):533-542.

Bowers, M. R., Noon, C. E., Wu, W., and Bass, J. K. (2016). Neonatal Physician Scheduling at the University of Tennessee Medical Center. Interfaces, 46(2):168182.

Bruni, R. and Detti, P. (2014). A flexible discrete optimization approach to the physician scheduling problem. Operations Research for Health Care, 3(4):191199.

Brunner, J. O. (2010). Flexible shift planning in the service industry: the case of physicians in hospitals, volume 640. Springer Science \& Business Media.

Brunner, J. O., Bard, J. F., and Kolisch, R. (2009). Flexible shift scheduling of physicians. Health Care Management Science, 12(3):285-305.

Brunner, J. O., Bard, J. F., and Kolisch, R. (2011). Midterm scheduling of physicians with flexible shifts using branch and price. IIE Transactions, 43(2):84109.

Brunner, J. O. and Edenharter, G. M. (2011). Long term staff scheduling of physicians with different experience levels in hospitals using column generation. Health Care Management Science, 14(2):189-202.

Burke, E. K., De Causmaecker, P., Berghe, G. V., and Van Landeghem, H. (2004). The State of the Art of Nurse Rostering. Journal of Scheduling, 7(6):441-499.

Carrasco, R. C. (2010). Long-term staff scheduling with regular temporal distribution. Computer Methods and Programs in Biomedicine, 100(2):191-199. 
Carter, M. W. and Lapierre, S. D. (2001). Scheduling emergency room physicians. Health Care Management Science, 4(4):347-360.

Cheang, B., Li, H., Lim, A., and Rodrigues, B. (2003). Nurse rostering problems-a bibliographic survey. European Journal of Operational Research, 151(3):447460.

Cohn, A., Root, S., Kymissis, C., Esses, J., and Westmoreland, N. (2009). Scheduling medical residents at boston university school of medicine. Interfaces, 39(3):186-195.

da Silva Rocha, M. S. F. (2013). The staff scheduling problem: a general model and applications. $\mathrm{PhD}$ thesis, Engineering and technology - Porto University.

Daldoul, D., Nouaouri, I., Bouchriha, H., and Allaoui, H. (2018). A stochastic model to minimize patient waiting time in an emergency department. Operations Research for Health Care, 18:16-25.

Day, T. E., Napoli, J. T., and Kuo, P. C. (2006). Scheduling the Resident 80-Hour Work Week: An Operations Research Algorithm. Current Surgery, 63(2):136141.

EL-Rifai, O., Garaix, T., Augusto, V., and Xie, X. (2015). A stochastic optimization model for shift scheduling in emergency departments. Health Care Management Science, 18(3):289-302.

Elomri, A., Elthlatiny, S., and Sidi Mohamed, Z. (2015). A goal programming model for fairly scheduling medicine residents. International Journal of Supply Chain Management, 4(2).

Erhard, M., Schoenfelder, J., Fügener, A., and Brunner, J. O. (2018). State of the art in physician scheduling. European Journal of Operational Research, 265(1):1-18.

Ernst, A., Jiang, H., Krishnamoorthy, M., and Sier, D. (2004). Staff scheduling and rostering: A review of applications, methods and models. European Journal of Operational Research, 153(1):3-27.

Ferrand, Y., Magazine, M., Rao, U. S., and Glass, T. F. (2011). Building Cyclic Schedules for Emergency Department Physicians. Interfaces, 41(6):521-533.

Firestone, M., Fenner-Crisp, P., Barry, T., Bennett, D., Chang, S., Callahan, M., Burke, A., Michaud, J., Olsen, M., Cirone, P., et al. (1997). Guiding principles for monte carlo analysis. Washington, DC: US Environmental Protection Agency. 
Fries, B. E. (1976). Bibliography of Operations Research in Health-Care Systems. Operations Research, 24(5):801-814.

Fügener, A., Brunner, J. O., and Podtschaske, A. (2015). Duty and workstation rostering considering preferences and fairness: a case study at a department of anaesthesiology. International Journal of Production Research, 53(24):74657487.

Ganguly, S., Lawrence, S., and Prather, M. (2014). Emergency Department Staff Planning to Improve Patient Care and Reduce Costs. Decision Sciences, 45(1):115-145.

Gendron, B., Lebbah, H., and Pesant, G. (2005). Improving the Cooperation Between the Master Problem and the Subproblem in Constraint Programming Based Column Generation. In International Conference on Integration of Artificial Intelligence (AI) and Operations Research (OR) Techniques in Constraint Programming, pages 217-227.

Glasserman, P. (2013). Monte Carlo methods in financial engineering, volume 53. Springer Science \& Business Media.

Gondane, M. S. and Zanwar, D. R. (2012). Staff Scheduling in Health Care Systems. IOSR Journal of Mechanical and Civil Engineering, 1:28-40.

Granja, C., Almada-Lobo, B., Janela, F., Seabra, J., and Mendes, A. (2014). An Optimization based on Simulation Approach to the Patient Admission Scheduling Problem: Diagnostic Imaging Department Case Study. Journal of Digital Imaging, 27(1):33-40.

Green, L. V. (2005). Capacity planning and management in hospitals. In Operations research and health care, pages 15-41. Springer.

Güler, M. G. (2013). A hierarchical goal programming model for scheduling the outpatient clinics. Expert Systems with Applications, 40(12):4906-4914.

Güler, M. G., Idin, K., and Yilmaz Güler, E. (2013). A goal programming model for scheduling residents in an anesthesia and reanimation department. Expert Systems with Applications, 40(6):2117-2126.

Gunawan, A. and Lau, H. C. (2010). The bi-objective master physician scheduling problem.

Gunawan, A. and Lau, H. C. (2013). Master physician scheduling problem. Journal of the Operational Research Society, 64(3):410-425. 
Gupta, D. and Denton, B. (2008). Appointment scheduling in health care: Challenges and opportunities. IIE transactions, 40(9):800-819.

Hans, E. W., Van Houdenhoven, M., and Hulshof, P. J. (2012). A framework for healthcare planning and control. In Handbook of healthcare system scheduling, pages 303-320. Springer.

Hidri, L. and Labidi, M. (2016). Optimal Physicians Schedule in an Intensive Care Unit. Advanced Science, Engineering and Medicine, 8(10):814-818.

Homem-de Mello, T., De Matos, V. L., and Finardi, E. C. (2011). Sampling strategies and stopping criteria for stochastic dual dynamic programming: a case study in long-term hydrothermal scheduling. Energy Systems, 2(1):1-31.

Huang, Y.-C., Lee, P.-T., and Huang, T.-L. (2016). A rostering optimization model for physician scheduling in medical department: a case study in district hospital. Journal of Industrial and Production Engineering, 33(8):533-557.

Hung, G. R., Whitehouse, S. R., O'neill, C., Gray, A. P., and Kissoon, N. (2007). Computer modeling of patient flow in a pediatric emergency department using discrete event simulation. Pediatric Emergency Care, 23(1):5-10.

Huntington, D. and Lyrintzis, C. (1998). Improvements to and limitations of latin hypercube sampling. Probabilistic Engineering Mechanics, 13(4):245-253.

Kaut, M. and Wallace, S. W. (2007). Evaluation of scenario-generation methods for stochastic programming. Pacific Journal of Optimization, 3(2):257-271.

Kazemian, P., Dong, Y., Rohleder, T. R., Helm, J. E., and Van Oyen, M. P. (2014). An IP-based healthcare provider shift design approach to minimize patient handoffs. Health Care Management Science, 17(1):1-14.

Kazemian, P., Sir, M. Y., Van Oyen, M. P., Lovely, J. K., Larson, D. W., and Pasupathy, K. S. (2017). Coordinating clinic and surgery appointments to meet access service levels for elective surgery. Journal of Biomedical Informatics, 66:105-115.

Kleywegt, A. J., Shapiro, A., and Homem-de Mello, T. (2002). The sample average approximation method for stochastic discrete optimization. SIAM Journal on Optimization, 12(2):479-502.

Kroese, D. P., Taimre, T., and Botev, Z. I. (2013). Handbook of monte carlo methods, volume 706. John Wiley \& Sons. 
Kuo, Y. H. (2014). Integrating simulation with simulated annealing for scheduling physicians in an understaffed emergency department. HKIE Transactions, 21(4):253-261.

Linderoth, J., Shapiro, A., and Wright, S. (2006). The empirical behavior of sampling methods for stochastic programming. Annals of Operations Research, 142(1):215-241.

Lo, C.-C. and Lin, T.-H. (2011). A Particle Swarm Optimization approach for physician scheduling in a hospital emergency department. In Natural Computation (ICNC), 2011 Seventh International Conference on, volume 4, pages 1929-1933. IEEE.

Mak, W.-K., Morton, D. P., and Wood, R. K. (1999). Monte carlo bounding techniques for determining solution quality in stochastic programs. Operations research letters, 24(1-2):47-56.

Martinez, G., Bernard, B. J., Larson, D. W., Pasupathy, K. S., and Sir, M. Y. (2016a). A data-driven approach for better assignment of clinical and surgical capacity in an elective surgical practice. In AMIA Annual Symposium Proceedings, volume 2016, page 874. American Medical Informatics Association.

Martinez, G., Huschka, T., Pasupathy, K., et al. (2016b). A coordinated scheduling policy to improve patient access to surgical services. In Proceedings of the 2016 Winter Simulation Conference, pages 2041-2052. IEEE Press.

Min, D. and Yih, Y. (2010). Scheduling elective surgery under uncertainty and downstream capacity constraints. European Journal of Operational Research, 206(3):642-652.

Moher, D., Liberati, A., Tetzlaff, J., and Altman, D. G. (2009). Preferred reporting items for systematic reviews and meta-analyses: the prisma statement. Annals of internal medicine, 151(4):264-269.

Norkin, V. I., Pflug, G. C., and Ruszczyński, A. (1998). A branch and bound method for stochastic global optimization. Mathematical Programming, 83(13):425-450.

Pengelly, J. (2002). Monte carlo methods. University of Otago.

Rosocha, L., Vernerova, S., and Verner, R. (2015). Medical Staff Scheduling Using Simulated Annealing. Quality Innovation Prosperity, 19(1):1-8. 
Savage, D. W., Woolford, D. G., Weaver, B., and Wood, D. (2015). Developing emergency department physician shift schedules optimized to meet patient demand. CJEM, 17(01):3-12.

Scopus (2017). Scopus [database online], https://www.scopus.com/search/form.uri?display=basic; accessed 1 june 2017.

Shamia, O., Aboushaqrah, N., and Bayoumy, N. (2015). Physician on call scheduling: Case of a Qatari hospital. In 2015 6th International Conference on Modeling, Simulation, and Applied Optimization (ICMSAO), pages 1-6. IEEE, IEEE.

Shapiro, A. and Homem-de Mello, T. (1998). A simulation-based approach to two-stage stochastic programming with recourse. Mathematical Programming, 81(3):301-325.

Sherali, H. D., Ramahi, M. H., and Saifee, Q. J. (2002). Hospital resident scheduling problem. Production Planning \& Control, 13(2):220-233.

Shields, M. D. and Zhang, J. (2016). The generalization of latin hypercube sampling. Reliability Engineering \& System Safety, 148:96-108.

Sinreich, D. and Jabali, O. (2007). Staggered work shifts: a way to downsize and restructure an emergency department workforce yet maintain current operational performance. Health care management science, 10(3):293-308.

Sinreich, D., Jabali, O., and Dellaert, N. P. (2012). Reducing emergency department waiting times by adjusting work shifts considering patient visits to multiple care providers. lie Transactions, 44(3):163-180.

Smalley, H. K. and Keskinocak, P. (2016). Automated medical resident rotation and shift scheduling to ensure quality resident education and patient care. Health Care Management Science, 19(1):66-88.

Smalley, H. K., Keskinocak, P., and Vats, A. (2015). Physician Scheduling for Continuity: An Application in Pediatric Intensive Care. Interfaces, 45(2):133148.

Stolletz, R. and Brunner, J. O. (2012). Fair optimization of fortnightly physician schedules with flexible shifts. European Journal of Operational Research, 219(3):622-629. 
Topaloglu, S. (2006). A multi-objective programming model for scheduling emergency medicine residents. Computers \& Industrial Engineering, 51(3):375388.

Topaloglu, S. (2009). A shift scheduling model for employees with different seniority levels and an application in healthcare. European Journal of Operational Research, 198(3):943-957.

Topaloglu, S. and Ozkarahan, I. (2011). A constraint programming-based solution approach for medical resident scheduling problems. Computers \& Operations Research, 38(1):246-255.

Turhan, A. M. and Bilgen, B. (2017). Mixed integer programming based heuristics for the Patient Admission Scheduling problem. Computers \& Operations Research, 80:38-49.

Turner, J., Kim, K., Mehrotra, S., DaRosa, D. A., Daskin, M. S., and Rodriguez, H. E. (2013). Using optimization models to demonstrate the need for structural changes in training programs for surgical medical residents. Health Care Management Science, 16(3):217-227.

Van Huele, C. and Vanhoucke, M. (2014). Analysis of the Integration of the Physician Rostering Problem and the Surgery Scheduling Problem. Journal of Medical Systems, 38(6):43.

Vile, J. L., Gillard, J. W., Harper, P. R., and Knight, V. A. (2016). Time-dependent stochastic methods for managing and scheduling Emergency Medical Services. Operations Research for Health Care, 8:42-52.

Wang, C.-W., Sun, L.-M., Jin, M.-H., Fu, C.-J., Liu, L., Chan, C.-H., and Kao, C.-Y. (2007). A genetic algorithm for resident physician scheduling problem. In Proceedings of the 9th annual conference on Genetic and evolutionary computation - GECCO '07, page 2203, New York, New York, USA. ACM, ACM Press.

Welch, S., Augustine, J., Camargo Jr, C. A., and Reese, C. (2006). Emergency department performance measures and benchmarking summit. Academic Emergency Medicine, 13(10):1074-1080.

White, C. A. and White, G. M. (2003). Scheduling Doctors for Clinical Training Unit Rounds Using Tabu Optimization. In International Conference on the Practice and Theory of Automated Timetabling, pages 120-128. 


\section{A \\ Complete account of the studies screened, assessed for eligi- bility, and included in the literature review}

This appendix provides a complete account of the studies screened, assessed for eligibility, and included in our review, with reasons for exclusions at each stage. Of note, we adopt the Preferred Reporting Items for Systematic reviews and Meta-Analyses (PRISMA) guidelines [Moher et al., 2009].

For data collection, we used the Scopus database and performed a keyword-driven search strategy. In what concerns the Physician Scheduling Problem (PSP), our search spanned publications prior to January of 2017 and comprised the fields "title", "abstracts", and "keywords" with no limitations with regards to the field "journals". Moreover, the search was limited to papers written in English, Spanish, or Portuguese. Due to the relative scarcity of studies dealing with physician scheduling, our search for PSP papers was not limited by the number of citations.

Keywords included words directly related to physician scheduling (see items (i)-(iv) below) as well as words related to solution techniques (see item (vii) below). We also made a specific search for words related to techniques that address uncertainty (see item (viii) below). Finally, it is worthwhile to mention that an advanced search was also performed to retrieve different spelling occurrences of the keywords in both the singular and plural forms. The keywords were chosen to yield the following unified query, where the asterisk $(*)$ is used as a substitute for zero or more characters, and the question mark (?) is used as a substitute for a single character:

(i) ("physician* rostering" OR "physician* scheduling")

(ii) ("resident rostering" OR "resident scheduling" OR "resident physician* rostering" OR "resident physician* scheduling" OR "residents rostering" OR "residents scheduling" OR "residents physician* rostering" OR "residents physician* scheduling")

(iii) ("physician* " OR "residents physician*" OR "resident physician*" OR resident OR residents)

(iv) ("rostering" OR "scheduling" OR "shift* scheduling" OR "shift* rostering") 
Appendix A. Complete account of the studies screened, assessed for eligibility,

$(\mathrm{v})=(\mathrm{iii}) \mathrm{AND}(\mathrm{iv})$

$(\mathrm{vi})=(\mathrm{i})$ OR (ii) OR (v)

(vii) (*heuristic* OR optimi?ation OR "*integer program*" OR "math* program*" OR "linear program*" OR "network optimi?ation" OR "goal program*" OR "mixed integer program*" OR "multi-objective program*" OR "multi objective program*" OR "multiple objective program*" OR "multiobjective optimi?ation" OR "multi objective optimi?ation" OR "multiple objective optimi?ation*" OR "constrain* program*" OR "constrain* optimi?ation" OR "set covering" OR "set patitioning" OR "?agrangian heuristic" OR "column generation" OR "ant colony optimi?ation" OR "tabu search" OR "genetic algorithm*" OR "memetic algorithm*" OR "grasp" OR "GRASP" OR "neural networks" OR "branch-and-bound" OR " branch and bound" OR "branch-and-price" OR " branch and price" OR "branch-and-cut" OR " branch and cut" OR "simulated annealing" OR "artificial inteligence" OR fuzzy OR "greedy search")

(viii) ("stochastic programm*" OR "stochastic optmi?ation*" OR "robust programm*" OR "rosbust optmi?ation*" OR uncertain* OR "chance constrain*" OR "chance-constrain*")

(ix) $=($ vi) AND (vii) AND (viii)

For study selection, we formulated the following exclusion criteria: (1) study is not related to physician scheduling; (2) study refers to patient appointment; (3) study does not use optimization/operation research techniques; (4) physician scheduling is not the main subject of the study. Studies whose abstract did not meet any number of the above criteria were excluded from further analysis. The studies retained were read in full, and in doing so, we verified the need to define two additional exclusion criteria: (5) paper is not available online; (6) study characteristics do not fit the classification field proposed for the review discussion and the summary table proposed.

Figure 2 details the number of studies screened, assessed for eligibility, and included in our review, with reasons for exclusion at each stage. 
Appendix A. Complete account of the studies screened, assessed for eligibility, and included in the literature review

Records identified though database search $(n=289)$

Records after duplicates removed $(n=283)$

Records screened $(\mathrm{n}=283)$

Full-text articles assessed for eligibility $(n=80) \quad \longrightarrow$ Full-text articles excluded by exclusion criteria $(n=40)$

Studies included in qualitative synthesis $(n=40)$

Figure A.1: Stepwise data collection process for physician scheduling 
B

Detailed account of the constraints defined in the reviewed Physician Scheduling Problem formulations

This appendix provides a detailed account of the constraints defined in the reviewed Physician Scheduling Problem (PSP) formulations. 
Table B.1: Time related constraints for PSP

\begin{tabular}{|c|c|c|c|c|c|c|c|c|c|c|c|c|c|c|c|c|c|c|c|c|c|c|c|c|c|c|c|c|c|c|c|c|c|c|c|c|c|c|c|c|c|c|c|c|}
\hline \multirow{2}{*}{ ef. } & \multicolumn{2}{|c|}{ A } & \multicolumn{2}{|c|}{ B } & \multicolumn{2}{|c|}{$\mathrm{C}$} & \multicolumn{2}{|c|}{$\mathrm{D}$} & \multicolumn{2}{|c|}{$\mathrm{E}$} & \multicolumn{2}{|c|}{$\mathrm{F}$} & \multicolumn{2}{|c|}{$\mathrm{G}$} & \multicolumn{2}{|c|}{$\mathrm{H}$} & \multicolumn{2}{|c|}{ I } & \multicolumn{2}{|c|}{$\mathrm{J}$} & \multicolumn{2}{|c|}{$\mathrm{K}$} & \multicolumn{2}{|c|}{$\mathrm{L}$} & \multicolumn{2}{|c|}{ M } & \multicolumn{2}{|c|}{$\mathrm{N}$} & C & & $\mathrm{I}$ & D & C & 2 & $\mathrm{R}$ & 3 & S & & $\mathrm{T}$ & & $\mathrm{U}$ & & $\mathrm{V}$ & \\
\hline & & & $\mathrm{h}$ & & h & & $\mathrm{b}$ & & h & & & & h & & & & $\mathrm{h}$ & & 1 & & & & $\mathrm{~h}$ & & 11 & S & & & $\mathrm{h}$ & D & & $\mathrm{s}$ & & $\mathrm{s}$ & $\mathrm{h}$ & $\mathrm{s}$ & & & $\mathrm{h}$ & & $\mathrm{h}$ & & & \\
\hline Bard et al. [2013] & - & - & - & - & - & - & - & - & 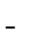 & - & 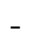 & . & 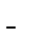 & . & 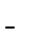 & - & - & - & - & & - & - & $\mathrm{x}$ & $\mathrm{x}$ & $\mathrm{x}$ & $\mathrm{x}$ & - & 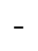 & - & - & 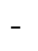 & - & - & - & . & - & - & 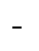 & - & - & - & 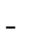 & - & 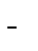 \\
\hline Bard et al. [2014] & - & - & - & - & - & - & - & - & - & & 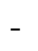 & - & . & - & 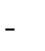 & - & - & - & - & & - & - & $\mathrm{x}$ & $\mathrm{x}$ & $\mathrm{x}$ & $\mathrm{x}$ & - & - & - & - & - & - & - & - & - & - & - & - & - & - & - & - & - & - \\
\hline Bard et al. [2016] & - & - & - & - & - & - & - & - & - & & - & - & - & - & - & - & - & - & - & & - & - & $\mathrm{x}$ & - & $\mathrm{x}$ & - & - & - & - & - & - & - & - & - & - & - & - & 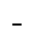 & - & - & - & - & - - & - \\
\hline Baum et al. [2014] & - & - & - & - & - & - & - & - & - & & - & - & - & - & - & - & - & - & $\mathrm{x}$ & - & - & - & $\mathrm{x}$ & - & - & - & - & - & - & - & - & - & - & - & - & - & - & - & - & - & - & - & - - & - \\
\hline Beaulieu et al. [2000] & $\mathrm{x}$ & - & - & - & - & $\mathrm{x}$ & - & - & - & - & - & - & - & $\mathrm{x}$ & - & - & $\mathrm{x}$ & - & - & - & - & - & - & - & - & - & - & - & - & - & - & $\mathrm{x}$ & - & $\mathrm{x}$ & - & - & - & - & - & - & - & - & - & - \\
\hline Bowers et al. [2016] & - & - & - & - & $\mathrm{x}$ & - & - & - & - & & - & - & - & - & - & - & $\mathrm{x}$ & - & - & - & - & - & $\mathrm{x}$ & - & - & - & - & - & - & - & - & - & - & - & - & - & - & - & - & - & - & - & - & - \\
\hline Bruni and Detti [2014] & $\mathrm{x}$ & - & - & - & - & - & - & - & $\mathrm{x}$ & & - & - & 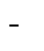 & - & - & - & - & - & - & - & . & - & - & - & - & - & $\mathrm{x}$ & - & $\mathrm{x}$ & - & - & - & - & - & - & - & - & - & - & - & - & - & - - & - \\
\hline Brunner and Edenharter [2011] & $\Lambda$ & - & - & - & - & - & - & - & - & 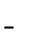 & 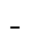 & - & - & - & - & - & - & - & - & - & - & - & $\mathrm{x}$ & - & - & - & 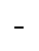 & - & - & - & $\mathrm{x}$ & - & - & - & - & - & 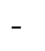 & 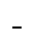 & $\mathrm{x}$ & - & $\mathrm{x}$ & - & - - & - \\
\hline Brunner et al. [2009] & $\mathrm{x}$ & - & - & - & - & - & - & - & - & - & - & - & - & - & - & - & - & - & - & - & - & - & - & $\mathrm{x}$ & - & - & - & - & - & - & - & - & - & $\mathrm{x}$ & - & - & - & - & $\mathrm{x}$ & - & $\mathrm{x}$ & - & $\mathrm{x}-$ & - \\
\hline Brunner et al. [2011] & $\mathrm{X}$ & - & $\mathrm{x}$ & - & - & - & - & - & - & - & $\mathrm{x}$ & - & - & - & - & - & - & - & - & - & - & - & $\mathrm{x}$ & - & - & - & - & - & - & - & - & - & - & $\mathrm{x}$ & - & - & - & - & $\mathrm{x}$ & - & $\mathrm{x}$ & - & $\mathrm{x}-$ & - \\
\hline Carrasco [2010] & $\mathrm{x}$ & - & - & - & - & - & - & - & - & 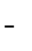 & - & - & - & - & - & - & - & - & - & - & - & - & - & - & - & - & - & - & - & - & - & - & - & - & - & - & - & - & - & - & - & - & - - & - \\
\hline Carter and Lapierre [2001] & $\mathrm{x}$ & $\mathrm{x}$ & - & - & $\mathrm{x}$ & $\mathrm{x}$ & $\mathrm{x}$ & $\mathrm{x}$ & $\mathrm{x}$ & $\mathrm{X}$ & - & - & - & - & - & - & - & - & - & & $\mathrm{x}$ & $\mathrm{x}$ & - & - & - & - & - & - & - & - & - & - & - & - & - & - & - & - & - & - & - & - & - & - \\
\hline Cohn et al. [2009] & 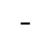 & - & - & - & - & - & - & - & - & & - & - & - & - & - & - & - & - & - & - & $\mathrm{x}$ & - & - & - & - & - & - & - & - & - & - & - & - & - & - & - & - & - & - & - & - & 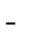 & - - & - \\
\hline Day et al. [2006] & $\mathrm{x}$ & - & - & - & - & - & $\mathrm{x}$ & - & - & - & - & - & - & - & $\mathrm{X}$ & - & - & - & - & - & - & - & - & - & - & - & - & - & $\mathrm{x}$ & - & - & - & $\mathrm{x}$ & - & - & - & - & - & - & - & - & - & - - & - \\
\hline Elomri et al. [2015] & - & - & - & - & $\mathrm{x}$ & - & - & - & - & & - & - & - & - & - & - & $\mathrm{x}$ & - & - & - & 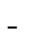 & - & - & - & - & - & - & - & - & - & - & - & - & - & - & - & - & - & - & - & - & 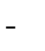 & - - & - \\
\hline Ferrand et al. [2011] & $\mathrm{x}$ & - & - & - & - & - & - & - & - & & - & $\mathrm{x}$ & $\mathrm{x}$ & - & - & - & - & - & - & - & $\mathrm{x}$ & - & $\mathrm{x}$ & - & $\mathrm{x}$ & - & - & - & - & - & - & - & - & - & - & - & - & - & - & - & - & - & - & - \\
\hline Fügener et al. [2015] & - & - & - & - & $\mathrm{x}$ & - & - & - & - & - & - & - & $\mathrm{x}$ & - & - & - & $\mathrm{x}$ & - & - & - & - & - & - & - & - & - & - & - & - & - & - & - & - & - & - & - & - & - & - & - & - & - & - & - \\
\hline Gendron et al. [2005] & $\mathrm{x}$ & - & - & - & - & - & - & - & - & - & - & - & - & - & - & - & $\mathrm{x}$ & - & - & - & - & - & - & - & - & - & - & - & - & - & - & $\mathrm{x}$ & - & $\mathrm{x}$ & - & - & - & - & - & - & - & - & - - & - \\
\hline Güler [2013] & - & - & - & - & - & - & - & - & - & & - & - & - & - & - & - & - & - & - & & $\mathrm{x}$ & - & - & - & - & - & - & - & $\mathrm{x}$ & - & - & - & - & - & - & - & - & - & - & - & - & 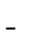 & - & - \\
\hline Güler et al. [2013] & - & - & - & - & $\mathrm{x}$ & - & - & - & - & & - & - & - & - & - & - & $\mathrm{x}$ & - & - & & - & - & $\mathrm{x}$ & - & - & - & - & - & - & - & - & - & - & - & - & $\mathrm{x}$ & - & - & - & - & - & 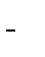 & - - & - \\
\hline Gunawan and Lau [2010] & 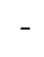 & - & - & - & - & - & - & - & 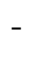 & & - & - & - & - & - & - & - & - & - & & - & - & $\mathrm{x}$ & - & 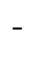 & - & 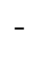 & - & - & - & - & - & - & - & - & - & - & - & - & - & - & 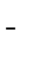 & - - & - \\
\hline Gunawan and Lau [2013] & - & - & - & - & - & - & 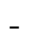 & - & - & & - & - & 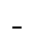 & - & . & - & $\mathrm{x}$ & - & - & & & - & - & - & - & - & - & - & - & - & - & - & & - & - & - & - & - & - & - & - & - & & \\
\hline
\end{tabular}

Gunawan and Lau [2013] 
Hidri and Labidi [2016]

Huang et al. [2016]

Kazemian et al. [2014]

Lo and Lin [2011]

Rosocha et al. [2015]

Savage et al. [2015]

Shamia et al. [2015]

Sherali et al. [2002]

Smalley and Keskinocak [2016]

Smalley et al. [2015]

Stolletz and Brunner [2012]

Topaloglu [2006]

Topaloglu [2009]

Topaloglu and Ozkarahan [2011]

Van Huele and Vanhoucke [2014]

Wang et al. [2007]

White and White [2003]

A - Minimum interval between shift allocations; B - Minimum interval after a block of shifts; C - Maximum consecutive working days; D - Minimum consecutive working days; E - Minimum consecutive days off; F - Maximum consecutive shift type; G - Minimum consecutive shift type; H - Maximum consecutive working hours; I - Maximum shift/block per day; J - Minimum shift/block per day; K - Maximum number of days on in a period; L - Maximum number of shifts worked during a period; $\mathrm{M}$ - Minimum number of shifts worked during a period; $\mathrm{N}$ - Maximum number of days off in a period; O - Minimum number of days off in a period; P - Minimum number of hours a physician must work in period; Q - Maximum number of hours a physician must work in period; R - Maximum number of assignments per week day; S - Maximum number of hours worked per day; T - Minimum length of time a shift must span; U - Maximum length of time a shift must span; V - Assigned the breaks required for each shift length. h - Hard constraints; s - Soft constraints. 
Table B.2: Weekend related constraints for PSP

\begin{tabular}{|c|c|c|c|c|c|c|c|c|c|c|c|c|c|c|}
\hline \multirow{2}{*}{ Ref. } & \multicolumn{2}{|c|}{$\mathrm{A}$} & \multicolumn{2}{|c|}{$\mathrm{B}$} & \multicolumn{2}{|c|}{$\mathrm{C}$} & \multicolumn{2}{|c|}{$\mathrm{D}$} & \multicolumn{2}{|c|}{$\mathrm{E}$} & \multicolumn{2}{|c|}{$\mathrm{F}$} & \multicolumn{2}{|c|}{$\mathrm{G}$} \\
\hline & $\mathrm{h}$ & $\mathrm{s}$ & $\mathrm{h}$ & $\mathrm{s}$ & $\mathrm{h}$ & s & $\mathrm{h}$ & $\mathrm{s}$ & $\mathrm{h}$ & $\mathrm{s}$ & $\mathrm{h}$ & s & $\mathrm{h}$ & $\mathrm{s}$ \\
\hline Beaulieu et al. [2000] & - & $\mathrm{x}$ & - & - & - & - & - & - & - & - & - & $\mathrm{x}$ & & - \\
\hline Bowers et al. [2016] & - & - & - & - & $\mathrm{x}$ & - & - & - & - & - & - & - & - & - \\
\hline Bruni and Detti [2014] & - & - & - & - & - & - & - & - & $\mathrm{x}$ & - & 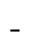 & - & - & - \\
\hline Carter and Lapierre [2001] & - & - & - & - & - & - & $\mathrm{x}$ & $\mathrm{x}$ & $\mathrm{x}$ & $\mathrm{x}$ & - & - & $\mathrm{x}$ & $\mathrm{x}$ \\
\hline Ferrand et al. [2011] & - & - & - & - & $\mathrm{x}$ & - & - & - & $\mathrm{x}$ & - & $\mathrm{x}$ & - & - & - \\
\hline Fügener et al. [2015] & - & - & - & - & - & - & $\mathrm{x}$ & - & 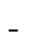 & - & - & - & - & - \\
\hline Güler et al. [2013] & - & - & - & - & $\mathrm{x}$ & - & - & - & - & - & t & $\mathrm{x}$ & - & - \\
\hline Hidri and Labidi [2016] & - & - & - & - & - & - & - & - & - & - & $\mathrm{x}$ & - & - & - \\
\hline Huang et al. [2016] & - & - & - & - & - & - & $\mathrm{x}$ & - & - & - & 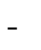 & - & - & - \\
\hline Rosocha et al. [2015] & - & - & - & - & - & - & - & $\mathrm{x}$ & $\mathrm{x}$ & $\mathrm{x}$ & - & $\mathrm{x}$ & - & - \\
\hline Sherali et al. [2002] & - & - & - & - & - & - & $\mathrm{x}$ & - & - & - & - & - & - & - \\
\hline Topaloglu [2006] & - & - & - & $\mathrm{x}$ & - & - & - & - & - & - & - & - & - & - \\
\hline Topaloglu [2009] & - & - & - & - & $\mathrm{x}$ & - & - & - & - & - & - & - & - & - \\
\hline Topaloglu and Ozkarahan [2011] & - & - & - & - & - & - & $\mathrm{x}$ & - & 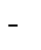 & - & 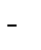 & - & - & - \\
\hline Van Huele and Vanhoucke [2014] & - & - & - & - & - & - & - & - & - & $\mathrm{x}$ & - & - & - & - \\
\hline
\end{tabular}

A - Number of complete working weekends; B - Number of weekend off in a period; C -Number of working weekend days; D - Maximum number of working weekends; E - Maximum number of consecutive work weekends; F - No split weekends; G - Work in identical shift types over a weekend. h - Hard constraints; s Soft constraints. 


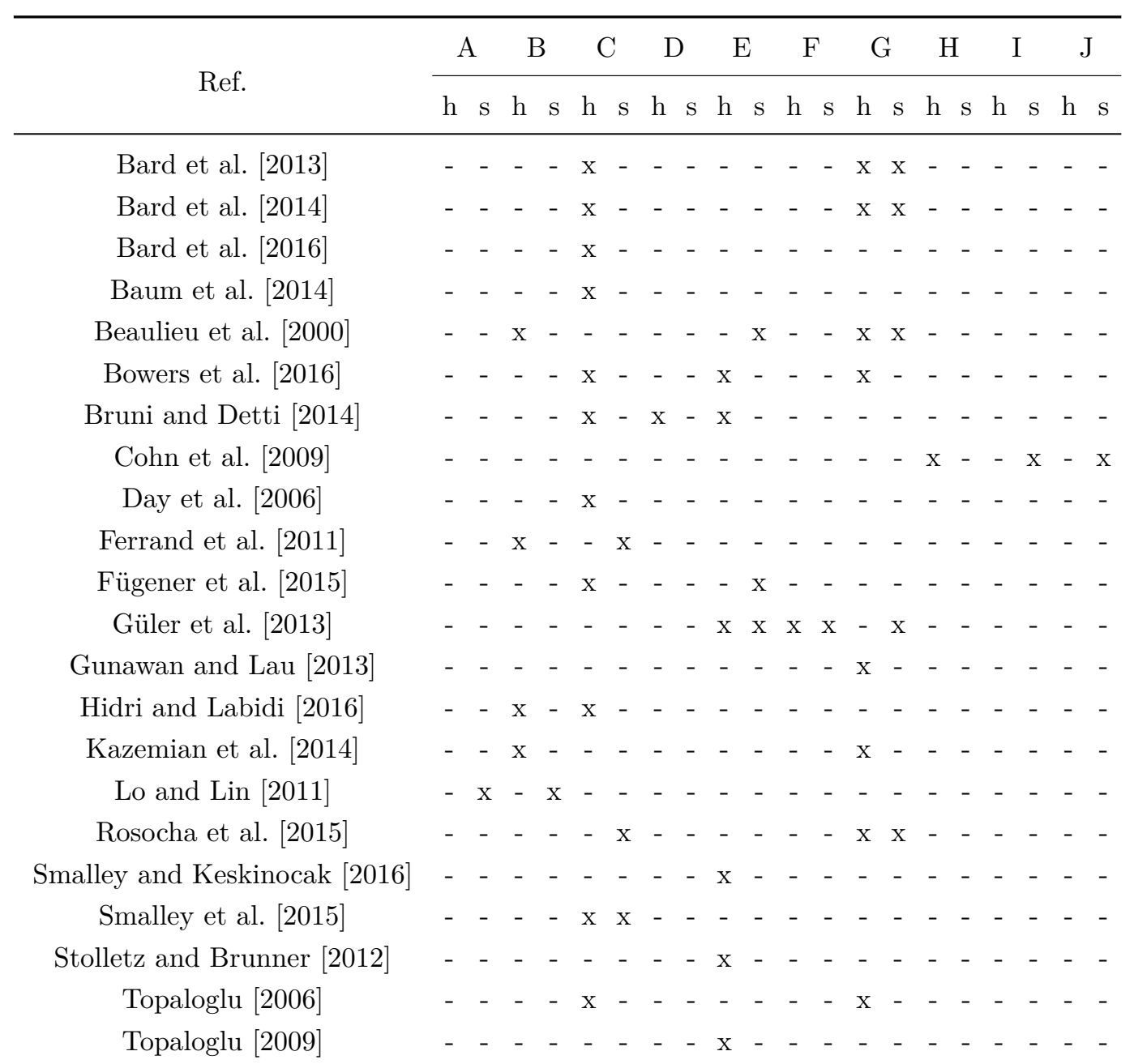

Topaloglu and Ozkarahan [2011] - - - - - - x - x - x - - - - - 
A - Off-on-off pattern; B - Minimum number of days off after night shifts or consecutive night shift; C - Assign specifics shifts patterns/to skill; D - No certain shift type for specific physician; E - Maximum number of certain shift type; F - Minimum number of certain shift type; G - Avoid some shifts patterns; H -

Residents cannot be assigned to calls at different hospitals on the same night; I - Assigned to work a shift on exactly one holiday during the period; J - Night assign location be the hospital closest to their daytime assignment. h - Hard constraints; s - Soft constraints.

Table B.4: Coverage related constraints for PSP

\begin{tabular}{|c|c|c|c|c|c|c|c|c|c|c|}
\hline \multirow{2}{*}{ Ref. } & \multicolumn{2}{|c|}{$\mathrm{A}$} & \multicolumn{2}{|c|}{ B } & \multicolumn{2}{|c|}{$\mathrm{C}$} & \multicolumn{2}{|c|}{$\mathrm{D}$} & \multicolumn{2}{|c|}{$\mathrm{E}$} \\
\hline & $\mathrm{h}$ & s & $\mathrm{h}$ & s & $\mathrm{h}$ & & $\mathrm{h}$ & & $\mathrm{h}$ & $\mathrm{s}$ \\
\hline Bard et al. [2013] & $\mathrm{x}$ & - & - & $\mathrm{x}$ & $\mathrm{x}$ & - & - & - & - & - \\
\hline Bard et al. [2014] & $\mathrm{x}$ & - & - & $\mathrm{x}$ & - & - & - & - & - & - \\
\hline Bard et al. [2016] & - & - & $\mathrm{x}$ & - & $\mathrm{x}$ & - & - & - & - & - \\
\hline Baum et al. [2014] & - & - & $\mathrm{x}$ & - & $\mathrm{x}$ & - & - & - & - & - \\
\hline Beaulieu et al. [2000] & $\mathrm{x}$ & - & - & - & - & - & - & - & - & - \\
\hline Bowers et al. [2016] & $\mathrm{x}$ & - & - & - & - & - & - & - & - & - \\
\hline Bruni and Detti [2014] & $\mathrm{x}$ & - & - & - & - & - & - & - & - & - \\
\hline Brunner and Edenharter [2011] & $\mathrm{x}$ & - & - & - & - & - & - & $\mathrm{x}$ & - & - \\
\hline Brunner et al. [2009] & $\mathrm{x}$ & - & - & - & - & - & - & - & - & - \\
\hline Brunner et al. [2011] & - & - & - & - & - & - & - & - & - & - \\
\hline Carrasco [2010] & $\mathrm{x}$ & - & - & - & - & - & $\mathrm{x}$ & - & - & - \\
\hline Carter and Lapierre [2001] & $\mathrm{x}$ & $\mathrm{x}$ & - & - & - & - & - & - & - & - \\
\hline Cohn et al. [2009] & $\mathrm{x}$ & - & - & - & - & - & - & - & - & - \\
\hline Day et al. [2006] & $\mathrm{x}$ & - & - & - & - & - & $\mathrm{x}$ & - & - & - \\
\hline
\end{tabular}


Ferrand et al. [2011]

Fügener et al. [2015]

Gendron et al. [2005]

Güler [2013]

Güler et al. [2013]

Gunawan and Lau [2010]

Hidri and Labidi [2016]

Huang et al. [2016]

Kazemian et al. [2014]

Lo and Lin [2011]

Rosocha et al. [2015]

Savage et al. [2015]

Sherali et al. [2002]

Smalley and Keskinocak [2016]

Smalley et al. [2015]

Stolletz and Brunner [2012]

Topaloglu [2006]

Topaloglu and Ozkarahan [2011

Turner et al. [2013]

Van Huele and Vanhoucke [2014]

Wang et al. [2007]

White and White [2003]

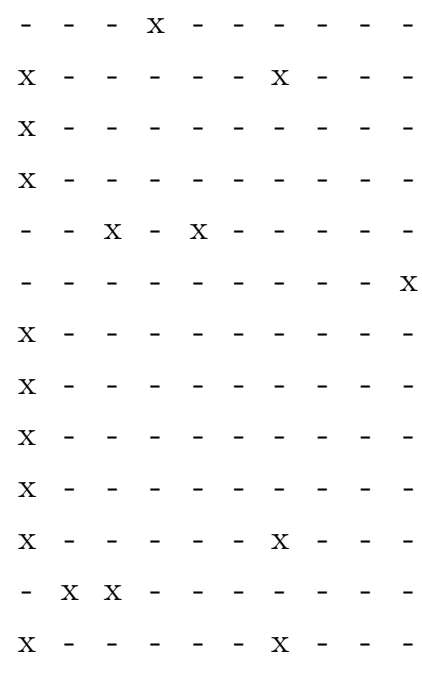

$--x-x--$

$\mathrm{x}-\mathrm{C}_{-}-\mathrm{C}_{-}-$

$\mathrm{x}-\mathrm{C}^{-}-\mathrm{C}^{-}-$

$-\mathrm{x}-\mathrm{x}_{-}-\mathrm{-}_{-}-$

$-\quad-\quad-\quad--$

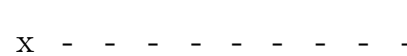

$-\quad-\quad-\quad-x--$

A - Cover required; B - Minimum staff level; C - Maximum staff level; D - Assign specific skill category per shift; E - Maximum number of physician for each period of work. h - Hard constraints; s - Soft constraints. 
Table B.5: Preference/Fairness related constraints for PSP

\begin{tabular}{|c|c|c|c|c|c|c|c|c|c|c|c|c|c|c|}
\hline \multirow{2}{*}{ Ref. } & \multicolumn{2}{|c|}{$\mathrm{A}$} & \multicolumn{2}{|c|}{$\mathrm{B}$} & \multicolumn{2}{|c|}{$\mathrm{C}$} & \multicolumn{2}{|c|}{$\mathrm{D}$} & \multicolumn{2}{|c|}{$\mathrm{E}$} & \multicolumn{2}{|c|}{$\mathrm{F}$} & \multicolumn{2}{|c|}{$\mathrm{G}$} \\
\hline & $\mathrm{h}$ & $\mathrm{s}$ & n & $\mathrm{s}$ & $\mathrm{h}$ & $\mathrm{s}$ & $\mathrm{h}$ & & $\mathrm{h}$ & $\mathrm{S}$ & $\mathrm{h}$ & & $\mathrm{h}$ & $\mathrm{s}$ \\
\hline Bard et al. [2016] & - & - & - & - & - & - & $\mathrm{x}$ & - & - & - & - & - & - & - \\
\hline Baum et al. [2014] & - & $\mathrm{x}$ & - & - & - & $\mathrm{x}$ & - & - & - & - & - & - & - & - \\
\hline Beaulieu et al. [2000] & - & - & $X$ & - & - & - & - & - & - & - & - & - & - & - \\
\hline Bowers et al. [2016] & $\mathrm{x}$ & - & $\mathrm{x}$ & $\mathrm{x}$ & - & - & 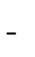 & - & - & - & - & - & - & - \\
\hline Bruni and Detti [2014] & - & $\mathrm{x}$ & - & - & - & - & - & - & - & 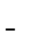 & - & 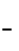 & - & - \\
\hline Brunner and Edenharter [2011] & - & - & - & - & - & - & - & - & - & - & - & & 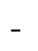 & $\mathrm{x}$ \\
\hline Brunner et al. [2009] & - & - & - & - & - & - & - & - & - & - & - & - & - & $\mathrm{x}$ \\
\hline Brunner et al. [2011] & - & - & - & - & - & - & - & - & - & - & - & - & - & $\mathrm{x}$ \\
\hline Carrasco $[2010]$ & $\mathrm{x}$ & $\mathrm{x}$ & - & - & - & - & - & 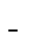 & - & - & - & - & - & - \\
\hline Carter and Lapierre [2001] & $\mathrm{x}$ & $\mathrm{x}$ & - & $\mathrm{x}$ & - & - & - & 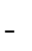 & - & - & & - & - & - \\
\hline Cohn et al. [2009] & - & $\mathrm{x}$ & 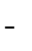 & $\mathrm{x}$ & - & - & - & - & - & - & $\lambda$ & & - & - \\
\hline Elomri et al. [2015] & - & $\mathrm{x}$ & - & - & - & - & - & - & - & - & - & & - & - \\
\hline Ferrand et al. [2011] & $\mathrm{x}$ & - & - & - & - & - & - & - & - & - & - & - & - & - \\
\hline Fügener et al. [2015] & - & $\mathrm{x}$ & - & $\mathrm{x}$ & - & - & - & - & - & - & - & - & - & - \\
\hline Gendron et al. [2005] & - & $\mathrm{x}$ & X & - & - & - & - & - & - & - & - & - & - & \\
\hline Güler [2013] & - & - & - & $\mathrm{x}$ & - & - & - & - & - & - & - & - & - & - \\
\hline Güler et al. [2013] & - & - & - & $\mathrm{x}$ & - & - & - & - & - & - & - & - & - & - \\
\hline Gunawan and Lau [2010] & - & $\mathrm{x}$ & - & $\mathrm{x}$ & - & - & - & - & - & - & - & & - & - \\
\hline Gunawan and Lau [2013] & - & - & & $\mathrm{x}$ & - & - & - & - & - & - & - & & - & - \\
\hline Hidri and Labidi [2016] & - & - & & - & - & - & 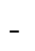 & - & - & - & & & - & \\
\hline Huang et al. [2016] & - & - & - & $\mathrm{x}$ & - & - & - & - & - & - & - & - & - & \\
\hline Kazemian et al. [2014] & - & - & - & - & - & - & $\mathrm{x}$ & - & - & - & 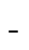 & - & - & \\
\hline
\end{tabular}

Kazemian et al. [2014] 
Lo and Lin [2011]

Rosocha et al. [2015]

Shamia et al. [2015]

Sherali et al. [2002]

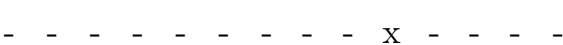

$--\mathrm{x}-\mathrm{C}_{-}-\mathrm{-}_{-}-{ }_{-}$

Smalley and Keskinocak [2016] - $\mathrm{x}$ - $\mathrm{x}$ - - - - - - - - -

Smalley et al. [2015] $\quad-\quad-\mathrm{x}$ x - - - - -

Stolletz and Brunner [2012] - $\mathrm{x}-\mathrm{x}$ x - - - - - - $\mathrm{x}$

Topaloglu [2006] $\quad-\mathrm{x}-\mathrm{C}_{-}-\mathrm{C}_{\mathrm{C}} \mathrm{-}-\mathrm{-}^{-}$

Topaloglu [2009] $\quad$ x $\mathrm{x}-\mathrm{x}-\mathrm{C}_{-}-\mathrm{C}_{-}-{ }_{-}$

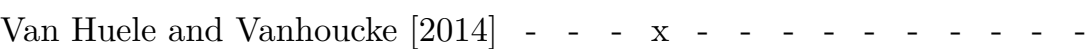

Wang et al. [2007] $\quad-\mathrm{x}-\mathrm{x}-\mathrm{x}_{-}-\mathrm{C}_{-}-\mathrm{-}_{-}$

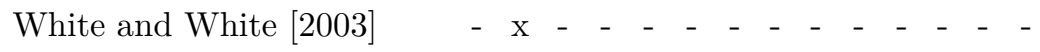

A - Balancing workload; B - Allocation according with the preferred schedule or shift rotational profile; C - Maximum overtime assigned to each physician; D Number of a shift type in a period for a given rotation should remain the same/Shift change is not allowed during bedside multidisciplinary rounds; E -

Schedules with few changes over time; F - No assign certain category in a specific day; G - All shift starting times in his or her schedule for an arbitrary week should be contained in an individual time window of pre-defined length. h - Hard constraints; s - Soft constraints.

Table B.6: Other constraints for PSP

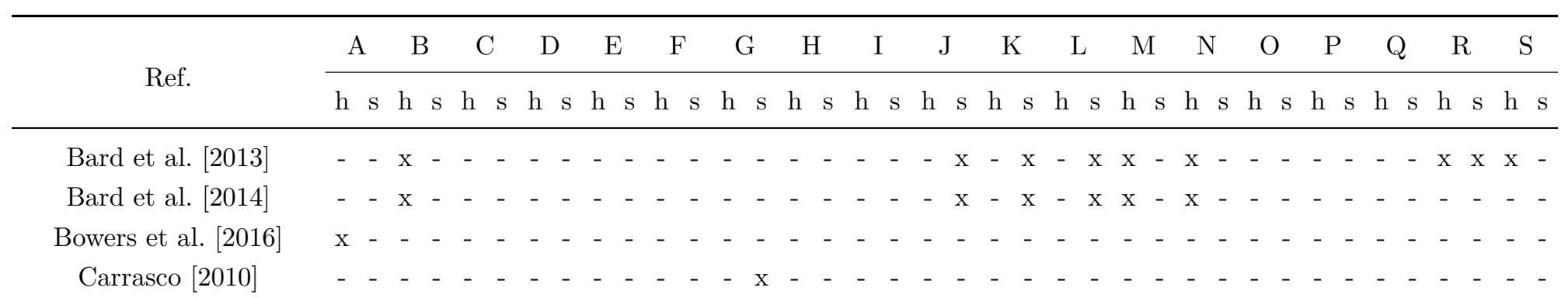


Cohn et al. [2009]

Elomri et al. [2015]

Güler [2013]

Güler et al. [2013]

$-$

$-$

Gunawan and Lau [2010]

Rosocha et al. [2015]

Smalley et al. [2015]

A - Continuity of staff when treating patients; B - Tutorship roster; C - Working separately; D - Each employee is assigned at most to one location and clinic type per time block per week; E - A clinic should be staffed with zero employees or with at least the minimum amount necessary to provide that type of clinic service; F - Prespecified number of shifts at each hospital; G - The group of employees assigned to the same shift, should change often and show no preference for specific pairings; $\mathrm{H}$ - Minimum duration that a resident has to work in a ward/clinic type; I - Minimum time residents work with each specialist; J - The ratio of house staff to faculty should be maximized within the given limits; K -Each resident should be assigned the same clinic days each week; L - Minimum number of faculty on duty each week; M - For each team at most max T team members can be assigned clinic duty on any day; N- Maximum assignment per area; O -

Maximum of duty per shift; P - Standard day-offs have to be determined before scheduling; Q - Each resident must perform all his duty in the same department in a period; R - Minimum house staff on a required inpatient rotation; S - No violation of ratio of house staff to faculty. $\mathrm{h}$ - Hard constraints; $\mathrm{s}$ - Soft constraints. 
C

Assessment of planning horizon, shift types, personnel characteristics, performance measures, and constraints found in the surveyed Physician Scheduling Problem papers

This appendix contains an assessment of planning horizon, shift types, personnel characteristics, performance measures, and constraints found in the surveyed Physician Scheduling Problem (PSP) papers. 
Table C.1: Planning horizon, shift types, and personnel characteristics found in surveyed PSP papers

\begin{tabular}{|c|c|c|c|c|c|c|c|c|c|c|c|c|c|c|}
\hline \multirow{2}{*}{ Ref. (A) } & \multirow{2}{*}{ PH (B) } & \multicolumn{2}{|c|}{ Shifts number (C) } & \multicolumn{2}{|c|}{ Shift length (D) } & \multicolumn{2}{|c|}{ Shift start (E) } & \multicolumn{2}{|c|}{ Shift overlapping $(\mathrm{F})$} & \multirow{2}{*}{ CS (G) } & \multicolumn{4}{|c|}{ Skill categories $(\mathrm{H})$} \\
\hline & & $123+$ & UD & $\mathrm{F}$ & $\mathrm{D}$ & $\mathrm{F}$ & $\mathrm{D}$ & $\mathrm{NO}$ & $\mathrm{O}$ & & 123 & + & SSep. & HSub. \\
\hline Bard et al. [2013] & UD & $--\mathrm{x}-$ & - & $\mathrm{x}$ & - & $\mathrm{x}$ & - & $\mathrm{x}$ & - & $\mathrm{x}$ & $\mathrm{x}--$ & - & $\mathrm{x}$ & - \\
\hline Bard et al. [2014] & $1 \mathrm{~m}$ & $-x--$ & - & $\mathrm{x}$ & - & $\mathrm{x}$ & - & $\mathrm{x}$ & - & - & $\mathrm{x}--$ & - & $\mathrm{x}$ & - \\
\hline Bard et al. [2016] & $1 \mathrm{~m}$ & $-x--$ & - & $\mathrm{x}$ & - & $\mathrm{x}$ & - & $\mathrm{x}$ & - & - & $\mathrm{x}--$ & - & $\mathrm{x}$ & - \\
\hline Baum et al. [2014] & $1 \mathrm{~m}$ & $-x--$ & - & $\mathrm{x}$ & - & $\mathrm{x}$ & - & $\mathrm{x}$ & - & - & $--x$ & - & $\mathrm{x}$ & - \\
\hline Bowers et al. [2016] & $4 \mathrm{w}$ & $--x$ & - & $\mathrm{x}$ & - & $\mathrm{x}$ & - & - & $\mathrm{x}$ & - & $\mathrm{x}--$ & - & $\mathrm{x}$ & - \\
\hline Bruni and Detti [2014] & $9 \mathrm{w}$ & $--\mathrm{x}-$ & - & $\mathrm{x}$ & - & $\mathrm{x}$ & - & - & $\mathrm{x}$ & - & $\mathrm{x}-{ }^{-}$ & - & $\mathrm{x}$ & - \\
\hline Brunner and Edenharter [2011] & UD & $-x--$ & - & $\mathrm{x}$ & - & $\mathrm{x}$ & - & $\mathrm{x}$ & - & - & -- & $\mathrm{x}$ & $\mathrm{x}$ & - \\
\hline Brunner et al. [2009] & $1 \mathrm{w}$ & --- & $\mathrm{x}$ & - & $\mathrm{x}$ & - & $\mathrm{x}$ & - & $\mathrm{x}$ & - & $-x-$ & - & - & $\mathrm{x}$ \\
\hline Brunner et al. [2011] & $2 \mathrm{w}$ & --- & $\mathrm{x}$ & - & $\mathrm{x}$ & - & $\mathrm{x}$ & - & $\mathrm{x}$ & - & $\mathrm{x}--$ & - & $\mathrm{x}$ & - \\
\hline Carrasco [2010] & UD & --- & $\mathrm{x}$ & - & $\mathrm{x}$ & - & $\mathrm{x}$ & - & $\mathrm{x}$ & - & $\mathrm{x}--$ & - & $\mathrm{x}$ & - \\
\hline Cohn et al. [2009] & $1 \mathrm{y}$ & $x---$ & - & $\mathrm{x}$ & - & $\mathrm{x}$ & - & $\mathrm{x}$ & - & - & $-x-$ & - & $\mathrm{x}$ & - \\
\hline Day et al. [2006] & $4 \mathrm{w}$ & $--x$ & - & $\mathrm{x}$ & - & $\mathrm{x}$ & - & $\mathrm{x}$ & - & - & -- & $\mathrm{x}$ & $\mathrm{x}$ & - \\
\hline Elomri et al. [2015] & $2 \mathrm{~m}$ & $-x--$ & - & $\mathrm{x}$ & - & $\mathrm{x}$ & - & - & $\mathrm{x}$ & - & $\mathrm{x}--$ & - & $\mathrm{x}$ & - \\
\hline Ferrand et al. [2011] & $1 y$ & $--x-$ & - & $\mathrm{x}$ & - & $\mathrm{x}$ & - & $\mathrm{x}$ & - & $\mathrm{x}$ & $\mathrm{x}--$ & - & $\mathrm{x}$ & - \\
\hline Fügener et al. [2015] & UD & $-x--$ & - & $\mathrm{x}$ & - & $\mathrm{x}$ & - & - & $\mathrm{x}$ & - & -- & $\mathrm{x}$ & $\mathrm{x}$ & - \\
\hline Gendron et al. [2005] & $2 \mathrm{w}$ & $--x$ & - & $\mathrm{x}$ & - & $\mathrm{x}$ & - & - & $\mathrm{x}$ & - & $\mathrm{x}--$ & - & $\mathrm{x}$ & - \\
\hline Güler [2013] & $1 \mathrm{~m}$ & $\mathrm{x}---$ & - & $\mathrm{x}$ & - & $\mathrm{x}$ & - & $\mathrm{x}$ & - & $\mathrm{x}$ & $-x-$ & - & $\mathrm{x}$ & - \\
\hline Güler et al. [2013] & $3 \mathrm{~m}$ & $-x--$ & - & $\mathrm{x}$ & - & $\mathrm{x}$ & - & $\mathrm{x}$ & - & - & --- & $\mathrm{x}$ & $\mathrm{x}$ & - \\
\hline Gunawan and Lau [2010] & $1 \mathrm{w}$ & $-x--$ & - & $\mathrm{x}$ & - & $\mathrm{x}$ & - & $\mathrm{x}$ & - & - & $\mathrm{x}-{ }^{-}$ & - & $\mathrm{x}$ & - \\
\hline
\end{tabular}

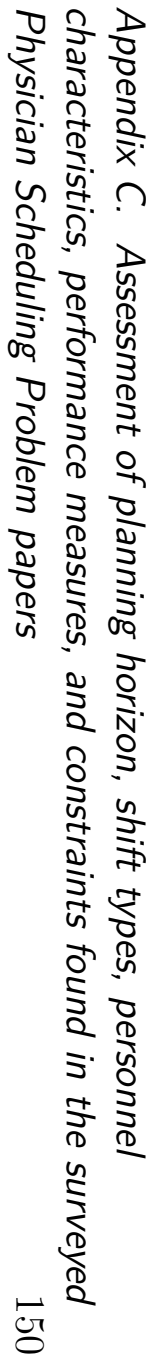


Gunawan and Lau [2013]

Hidri and Labidi [2016]

Huang et al. [2016]

Kazemian et al. [2014]

Lo and Lin [2011]

Rosocha et al. [2015]

Savage et al. [2015]

Shamia et al. [2015]

Sherali et al. [2002]

Smalley and Keskinocak [2016]

Smalley et al. [2015]

Stolletz and Brunner [2012]

Topaloglu [2006]

Topaloglu [2009]

Topaloglu and Ozkarahan [2011]

Turner et al. [2013]

Van Huele and Vanhoucke [2014]

Wang et al. [2007]

White and White [2003]

Ref. - Reference PH - Planning horizon; w - week(s); m - month; d - day(s); h - hours; y - year; UD - user-definable + More shifts; UD - user-definable F fixed; D - definable NO - non-overlapping; O - overlapping CS - Cyclical shifts SSep. - Schedule separately; HSub.- Hierarchical substitution allowed

Table C.2: Performance measures found in surveyed PSP papers

Objective

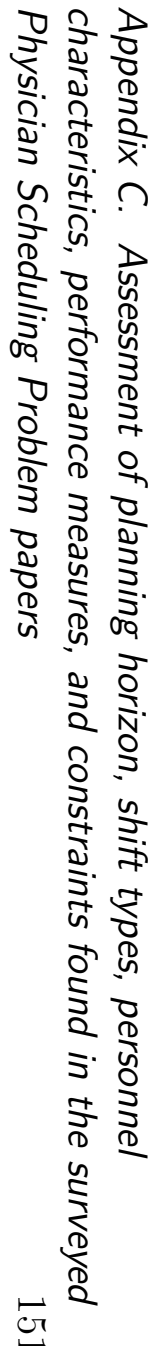


PUC-Rio - Certificação Digital No 1521900/CA

\begin{tabular}{|c|c|c|c|c|c|c|c|c|c|c|c|c|c|c|c|c|c|c|c|c|}
\hline Min/Max & Min & Max & Min & Min & Min & Min & $\operatorname{Max}$ & $\operatorname{Max}$ & Min & Min & Min & Min & Min & Min & Min & Min & Min & Min & Min & Min \\
\hline Bard et al. [2013] & $\mathrm{x}$ & - & - & - & - & - & - & - & - & - & - & - & - & - & - & - & - & - & - & - \\
\hline Bard et al. [2014] & $\mathrm{x}$ & - & - & - & - & - & $\mathrm{x}$ & - & - & - & - & - & - & - & - & - & - & - & - & - \\
\hline Bard et al. [2016] & - & - & - & - & - & - & $\mathrm{x}$ & - & - & - & - & - & - & - & - & - & - & - & - & - \\
\hline Baum et al. [2014] & - & - & - & - & - & - & - & - & - & - & - & - & - & - & - & - & - & $\mathrm{x}$ & $\mathrm{x}$ & - \\
\hline Beaulieu et al. [2000] & - & $\mathrm{x}$ & - & - & - & - & - & - & - & - & - & - & - & - & - & - & - & - & - & - \\
\hline Bowers et al. [2016] & $\mathrm{x}$ & - & - & - & - & - & - & - & - & - & - & - & - & - & - & - & - & - & - & - \\
\hline Bruni and Detti [2014] & - & $\mathrm{x}$ & - & - & - & - & - & - & - & - & - & - & - & - & - & - & - & - & - & - \\
\hline Brunner and Edenharter [2011] & - & $\mathrm{x}$ & $\mathrm{x}$ & - & - & - & - & $\mathrm{x}$ & - & - & - & - & - & - & - & - & - & - & - & - \\
\hline Brunner et al. [2009] & - & - & - & - & - & - & - & - & $\mathrm{x}$ & - & - & - & - & - & - & - & - & - & - & - \\
\hline Brunner et al. [2011] & - & - & - & $\mathrm{x}$ & $\mathrm{x}$ & - & - & - & - & - & - & - & - & - & - & - & - & - & - & - \\
\hline Carrasco $[2010]$ & - & - & - & $\mathrm{x}$ & $\mathrm{x}$ & - & - & - & - & - & - & - & - & - & - & - & - & - & - & - \\
\hline Carter and Lapierre [2001] & - & - & - & - & - & - & - & - & - & - & - & $\mathrm{x}$ & - & - & - & - & - & - & - & - \\
\hline Cohn et al. [2009] & - & - & $\mathrm{x}$ & - & $\mathrm{x}$ & - & - & - & - & - & - & - & - & - & - & - & - & - & - & - \\
\hline Day et al. [2006] & - & - & - & - & - & - & $\mathrm{x}$ & - & - & - & - & - & - & $\mathrm{x}$ & - & - & - & - & - & - \\
\hline Elomri et al. [2015] & - & - & - & - & - & - & - & $\mathrm{x}$ & - & - & - & - & - & - & - & - & - & - & - & - \\
\hline Ferrand et al. [2011] & $\mathrm{x}$ & - & - & - & - & - & - & - & - & - & - & - & - & - & - & - & - & - & - & - \\
\hline Fügener et al. [2015] & $\mathrm{x}$ & $\mathrm{x}$ & $\mathrm{x}$ & - & - & - & - & - & - & - & - & - & - & - & - & - & - & - & - & - \\
\hline Gendron et al. [2005] & - & - & - & - & - & - & - & $\mathrm{x}$ & - & - & - & - & - & - & - & - & - & - & - & - \\
\hline Güler [2013] & $\mathrm{x}$ & - & - & - & - & - & - & - & - & - & - & - & - & - & - & - & - & - & - & - \\
\hline Güler et al. [2013] & $\mathrm{x}$ & - & - & - & - & - & - & - & - & - & - & - & - & - & - & - & - & - & - & - \\
\hline Gunawan and Lau [2010] & - & $\mathrm{x}$ & - & - & - & - & - & - & - & - & - & - & - & - & - & $\mathrm{x}$ & - & - & - & - \\
\hline Gunawan and Lau [2013] & - & $\mathrm{x}$ & - & - & - & $\mathrm{x}$ & - & - & - & - & - & - & - & - & - & - & - & - & - & - \\
\hline Hidri and Labidi [2016] & - & - & - & $\mathrm{x}$ & - & - & - & - & - & - & - & - & - & - & - & - & - & - & - & - \\
\hline Huang et al. [2016] & - & $\mathrm{x}$ & - & - & - & - & - & - & - & - & - & - & - & - & - & - & - & - & - & - \\
\hline Kazemian et al. [2014] & - & - & - & - & - & - & - & - & - & $\mathrm{x}$ & - & - & - & - & - & - & - & - & - & - \\
\hline
\end{tabular}

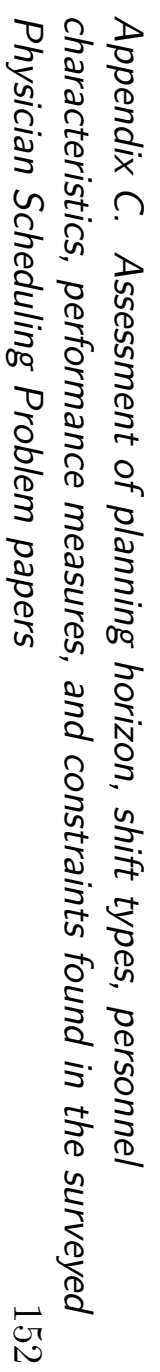


PUC-Rio - Certificação Digital No 1521900/CA

\begin{tabular}{|c|c|c|c|c|c|c|c|c|c|c|c|c|}
\hline Bard et al. [2013] & $\mathrm{x}$ & $\mathrm{x}$ & $\mathrm{x}$ & $\mathrm{x}$ & - & - & $\mathrm{x}$ & $\mathrm{x}$ & $\mathrm{x}$ & $\mathrm{x}$ & - & - \\
\hline Bard et al. [2014] & $\mathrm{x}$ & $\mathrm{x}$ & - & - & $\mathrm{x}$ & $\mathrm{x}$ & $\mathrm{x}$ & $\mathrm{x}$ & - & - & $\mathrm{x}$ & $\mathrm{x}$ \\
\hline Bard et al. [2016] & $\mathrm{x}$ & $\mathrm{x}$ & - & - & $\mathrm{x}$ & $\mathrm{x}$ & $\mathrm{x}$ & $\mathrm{x}$ & - & - & $\mathrm{x}$ & $\mathrm{x}$ \\
\hline Baum et al. [2014] & $\mathrm{x}$ & - & - & - & $\mathrm{x}$ & - & $\mathrm{x}$ & - & $\mathrm{x}$ & - & - & - \\
\hline Beaulieu et al. [2000] & $\mathrm{x}$ & - & - & - & $\mathrm{x}$ & - & $\mathrm{x}$ & - & - & $\mathrm{x}$ & - & - \\
\hline Bowers et al. [2016] & $\mathrm{x}$ & $\mathrm{x}$ & - & $\mathrm{x}$ & $\mathrm{x}$ & $\mathrm{x}$ & $\mathrm{x}$ & - & $\mathrm{x}$ & - & - & - \\
\hline Bruni and Detti [2014] & $\mathrm{x}$ & - & $\mathrm{x}$ & - & $\mathrm{x}$ & - & $\mathrm{x}$ & - & $\mathrm{x}$ & $\mathrm{x}$ & $\mathrm{x}$ & - \\
\hline Brunner and Edenharter [2011] & $\mathrm{x}$ & - & $\mathrm{x}$ & - & $\mathrm{x}$ & - & $\mathrm{x}$ & - & - & $\mathrm{x}$ & - & - \\
\hline Brunner et al. [2009] & $\mathrm{x}$ & - & - & - & - & - & $\mathrm{x}$ & $\mathrm{x}$ & - & $\mathrm{x}$ & - & - \\
\hline Brunner et al. [2011] & $\mathrm{x}$ & $\mathrm{x}$ & - & - & - & - & $\mathrm{x}$ & - & - & $\mathrm{x}$ & - & - \\
\hline Carrasco [2010] & $\mathrm{x}$ & $\mathrm{x}$ & - & - & - & - & $\mathrm{x}$ & - & - & $\mathrm{x}$ & - & - \\
\hline Carter and Lapierre [2001] & $\mathrm{x}$ & - & - & - & - & - & $\mathrm{x}$ & - & $\mathrm{x}$ & $\mathrm{x}$ & - & - \\
\hline Cohn et al. [2009] & $\mathrm{x}$ & - & - & - & $\mathrm{x}$ & - & $\mathrm{x}$ & - & $\mathrm{x}$ & $\mathrm{x}$ & - & - \\
\hline Day et al. [2006] & $\mathrm{x}$ & - & - & - & $\mathrm{x}$ & - & $\mathrm{x}$ & - & - & - & - & - \\
\hline Elomri et al. [2015] & $\mathrm{x}$ & - & - & - & - & - & - & - & - & $\mathrm{x}$ & $\mathrm{x}$ & - \\
\hline Ferrand et al. [2011] & $\mathrm{x}$ & $\mathrm{x}$ & $\mathrm{x}$ & - & $\mathrm{x}$ & $\mathrm{x}$ & - & $\mathrm{x}$ & $\mathrm{x}$ & - & - & - \\
\hline Fügener et al. [2015] & $\mathrm{x}$ & - & $\mathrm{x}$ & - & $\mathrm{x}$ & $\mathrm{x}$ & $\mathrm{x}$ & - & - & $\mathrm{x}$ & - & - \\
\hline Gendron et al. [2005] & $\mathrm{x}$ & $\mathrm{x}$ & - & - & - & - & $\mathrm{x}$ & - & $\mathrm{x}$ & $\mathrm{x}$ & - & - \\
\hline Güler [2013] & $\mathrm{x}$ & - & - & - & - & - & $\mathrm{x}$ & - & - & $\mathrm{x}$ & - & - \\
\hline Güler et al. [2013] & $\mathrm{x}$ & $\mathrm{x}$ & $\mathrm{x}$ & $\mathrm{x}$ & $\mathrm{x}$ & $\mathrm{x}$ & $\mathrm{x}$ & - & - & $\mathrm{x}$ & $\mathrm{x}$ & $\mathrm{x}$ \\
\hline Gunawan and Lau [2010] & $\mathrm{x}$ & - & - & - & - & - & - & $\mathrm{x}$ & - & $\mathrm{x}$ & $\mathrm{x}$ & - \\
\hline Gunawan and Lau [2013] & $\mathrm{x}$ & - & - & - & $\mathrm{x}$ & - & - & - & - & $\mathrm{x}$ & - & - \\
\hline Hidri and Labidi [2016] & $\mathrm{x}$ & - & $\mathrm{x}$ & - & $\mathrm{x}$ & - & $\mathrm{x}$ & - & - & - & - & - \\
\hline Huang et al. [2016] & $\mathrm{x}$ & - & $\mathrm{x}$ & - & - & - & $\mathrm{x}$ & - & - & $\mathrm{x}$ & - & - \\
\hline Kazemian et al. [2014] & $\mathrm{x}$ & - & - & - & $\mathrm{x}$ & - & $\mathrm{x}$ & - & $\mathrm{x}$ & - & - & - \\
\hline Lo and Lin [2011] & $\mathrm{x}$ & $\mathrm{x}$ & - & - & - & $\mathrm{x}$ & $\mathrm{x}$ & - & - & $\mathrm{x}$ & - & - \\
\hline
\end{tabular}

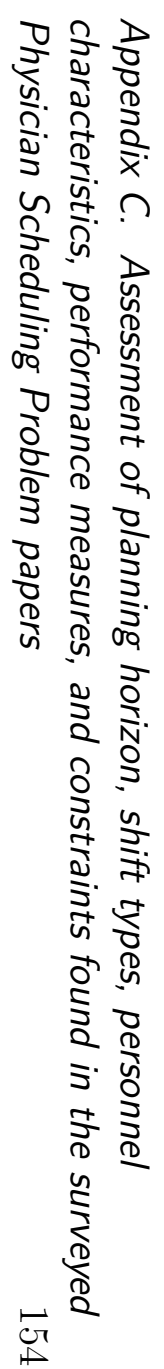


Rosocha et al. [2015]

Savage et al. [2015]

Shamia et al. [2015

Sherali et al. [2002]

Smalley and Keskinocak [2016]

Smalley et al. [2015]

Stolletz and Brunner [2012]

Topaloglu [2006]

Topaloglu [2009]

Turner et al. [2013]

Van Huele and Vanhoucke [2014]

Wang et al. [2007]

White and White [2003]
Topaloglu and Ozkarahan [2011]

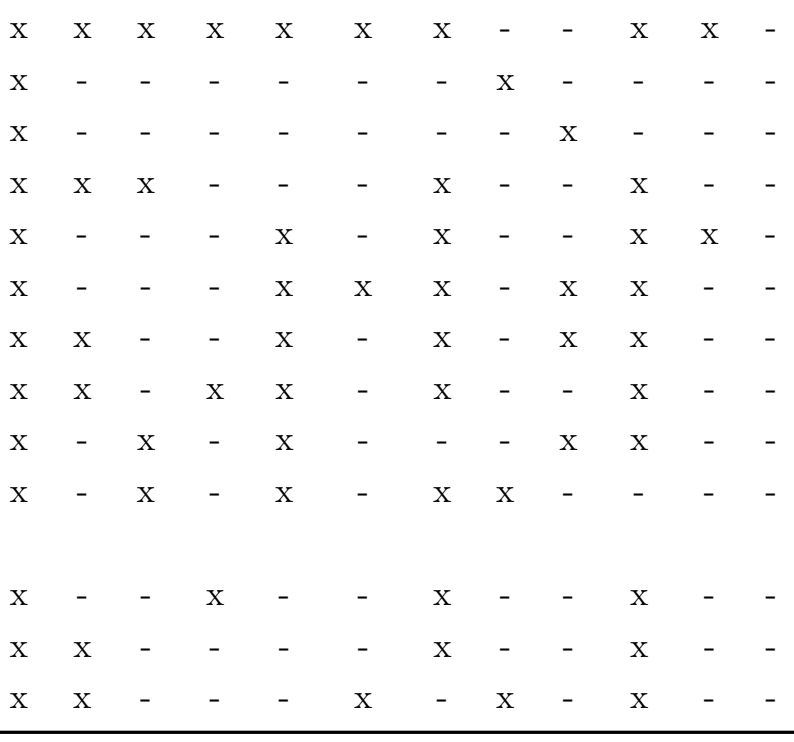

TR - Time related constrains; WR - Weekend related constrains; SP/S - Shifts pattern/sequencing constrains; CR - Cover related constraints; P/R Preference/Fairness related constraints; O - Other constraints; HC - Hard constraints; SC - Soft constraints

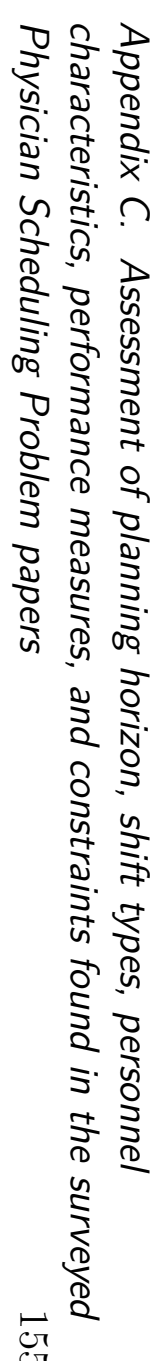


D

\section{Extra material from the Hospital A's case study from Chapter} 4

Table D.1: Current schedule of physicians in the Hospital A

\begin{tabular}{|c|c|c|c|c|c|c|c|c|c|c|c|c|c|c|c|c|c|c|c|c|c|c|c|c|c|c|c|c|}
\hline \multirow[b]{2}{*}{ Shift } & \multicolumn{7}{|c|}{ Week 1} & \multicolumn{7}{|c|}{ Week 2} & \multicolumn{7}{|c|}{ Week 3} & \multicolumn{7}{|c|}{ Week 4} \\
\hline & M & $\mathbf{T}$ & W & $\mathbf{T}$ & $\mathbf{F}$ & $\mathrm{S}$ & $\mathrm{S}$ & M & $\mathrm{T}$ & $\mathbf{W}$ & $\mathbf{T}$ & $\mathbf{F}$ & $\mathrm{S}$ & $\mathrm{S}$ & M & $T$ & W & $\mathrm{T}$ & F & $\mathrm{S}$ & $\mathrm{S}$ & $\mathrm{M}$ & $\mathbf{T}$ & $\mathbf{W}$ & $\mathrm{T}$ & F & $\mathrm{S}$ & $\mathrm{S}$ \\
\hline 1 & 5 & 5 & 5 & 5 & 5 & 5 & 2 & 5 & 5 & 5 & 5 & 5 & 4 & 4 & 5 & 5 & 5 & 5 & 5 & 5 & 4 & 5 & 5 & 5 & 5 & 5 & 4 & 5 \\
\hline 2 & 2 & 2 & 1 & 2 & 2 & 2 & 2 & 2 & 2 & 1 & 2 & 1 & 2 & 2 & 2 & 2 & 1 & 2 & 2 & 2 & 2 & 2 & 2 & 1 & 2 & 2 & 2 & 2 \\
\hline 3 & 2 & 1 & 2 & 1 & 2 & 3 & 3 & 2 & 1 & 2 & 2 & 1 & 3 & 3 & 2 & 1 & 2 & 1 & 2 & 3 & 3 & 2 & 1 & 2 & 2 & 1 & 3 & 3 \\
\hline 4 & 5 & 5 & 5 & 5 & 3 & 5 & 2 & 5 & 5 & 5 & 5 & 3 & 4 & 4 & 5 & 5 & 5 & 5 & 3 & 4 & 4 & 5 & 5 & 5 & 5 & 3 & 4 & 5 \\
\hline 5 & 2 & 2 & 2 & 1 & 1 & 2 & 2 & 2 & 2 & 2 & 1 & 0 & 2 & 2 & 2 & 2 & 2 & 1 & 1 & 2 & 2 & 2 & 2 & 2 & 1 & 0 & 2 & 2 \\
\hline 6 & 2 & 2 & 2 & 2 & 1 & 3 & 3 & 2 & 2 & 2 & 2 & 0 & 3 & 3 & 2 & 2 & 2 & 2 & 2 & 3 & 3 & 2 & 2 & 2 & 2 & 0 & 3 & 2 \\
\hline 7 & 3 & 4 & 4 & 3 & 3 & 4 & 3 & 3 & 4 & 4 & 4 & 4 & 1 & 3 & 3 & 4 & 4 & 4 & 3 & 3 & 3 & 3 & 4 & 4 & 3 & 3 & 3 & 2 \\
\hline
\end{tabular}

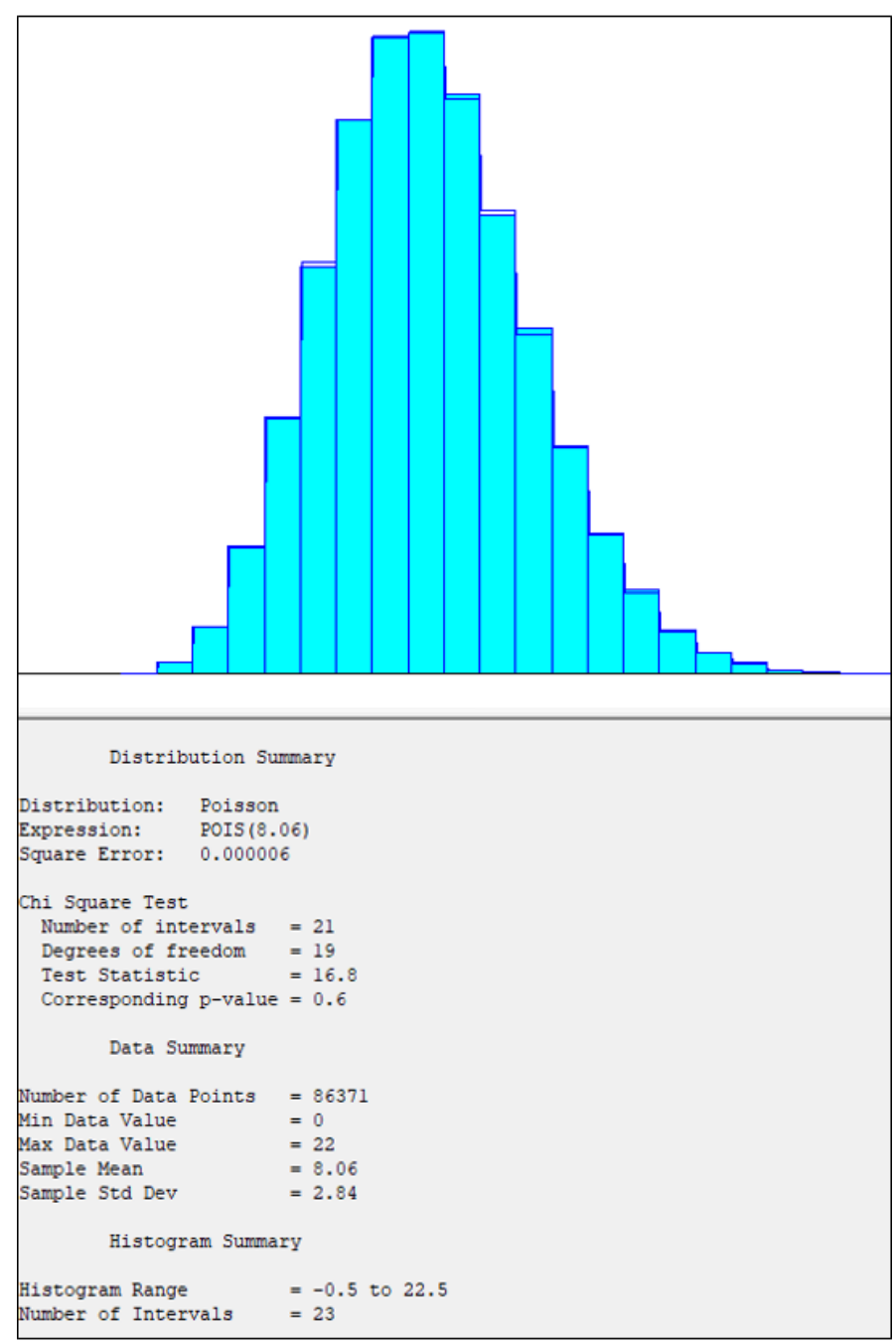

Figure D.1: Fit test of the patients arrival distribution in the Hospital A 
Appendix D. Extra material from the Hospital A's case study from Chapter147

Table D.2: Average interval between arrival per hour and day of week in ED of Hospital A

\begin{tabular}{|c|c|c|c|c|c|c|c|}
\hline \multirow[b]{2}{*}{ Time of day $(h)^{a}$} & \multicolumn{7}{|c|}{ Day } \\
\hline & $\mathbf{M}$ & $\mathbf{T}$ & $\mathbf{W}$ & $\mathbf{T}$ & $\mathbf{F}$ & $\mathbf{S}$ & $\mathbf{S}$ \\
\hline 0 & 15.0 & 11.3 & 13.9 & 12.2 & 14.3 & 12.7 & 13.7 \\
\hline 1 & 18.5 & 15.4 & 18.3 & 17.3 & 16.3 & 17.7 & 17.1 \\
\hline 2 & 24.9 & 22.2 & 25.1 & 25.7 & 24.2 & 23.0 & 23.6 \\
\hline $\begin{array}{l}3 \\
4 \\
5 \\
6\end{array}$ & 32.4 & 33.3 & 34.1 & 33.7 & 37.0 & 34.3 & 32.2 \\
\hline 7 & 17.7 & 16.8 & 18.3 & 20.0 & 20.1 & 21.5 & 25.4 \\
\hline $\begin{array}{c}8 \\
23\end{array}$ & 8.4 & 9.7 & 9.9 & 11.4 & 10.8 & 11.8 & 12.7 \\
\hline 9 & 6.0 & 6.5 & 6.8 & 7.2 & 8.0 & 8.4 & 7.9 \\
\hline $\begin{array}{l}10 \\
11\end{array}$ & 3.8 & 4.4 & 4.5 & 4.8 & 5.2 & 5.1 & 5.1 \\
\hline $\begin{array}{l}12 \\
14\end{array}$ & 4.3 & 4.8 & 5.2 & 5.3 & 5.8 & 5.5 & 5.3 \\
\hline $\begin{array}{l}13 \\
15\end{array}$ & 4.5 & 5.1 & 5.4 & 5.3 & 5.9 & 5.7 & 5.8 \\
\hline $\begin{array}{l}16 \\
17 \\
20\end{array}$ & 4.8 & 5.5 & 5.9 & 6.2 & 6.6 & 6.4 & 6.3 \\
\hline $\begin{array}{l}18 \\
21\end{array}$ & 5.1 & 5.8 & 6.4 & 6.7 & 7.6 & 7.5 & 6.7 \\
\hline
\end{tabular}

a24 hour clock format 
E

Size of the model from the Hospital A's case study from Chapter 4

The size of the models (i.e., variables and constraints), average execution times, and their standard deviation are shown in Table E.1.

Table E.1: Size of the model from the case study in the Hospital A

\begin{tabular}{ccccc}
\hline Scenarios & Variables & Constraints & Time(s) & St.Dev (s) \\
\hline $\mathbf{1 0 0}$ & 43373 & 53215 & 15.27 & 28.02 \\
$\mathbf{2 0 0}$ & 76973 & 70015 & 17.97 & 12.97 \\
$\mathbf{3 0 0}$ & 110573 & 86815 & 26.24 & 35.53 \\
$\mathbf{4 0 0}$ & 144173 & 103615 & 33.01 & 26.47 \\
$\mathbf{5 0 0}$ & 177773 & 120415 & 36.43 & 10.12 \\
$\mathbf{6 0 0}$ & 211373 & 137215 & 39.41 & 11.23 \\
$\mathbf{7 0 0}$ & 244973 & 154015 & 46.63 & 6.99 \\
$\mathbf{8 0 0}$ & 278573 & 170815 & 57.5 & 6.22 \\
$\mathbf{9 0 0}$ & 312173 & 187615 & 61.11 & 29.94 \\
$\mathbf{1 0 0 0}$ & 345773 & 204415 & 69.26 & 17.40 \\
\hline
\end{tabular}




\section{$\mathbf{F}$ \\ Extra SAA material from the Hospital A's case study}

Table F.1 shows the minimum and maximum confidence levels for the optimal gap in each experiment.

Table F.1: Confidence interval of the optimal gap from case study in the Hospital A

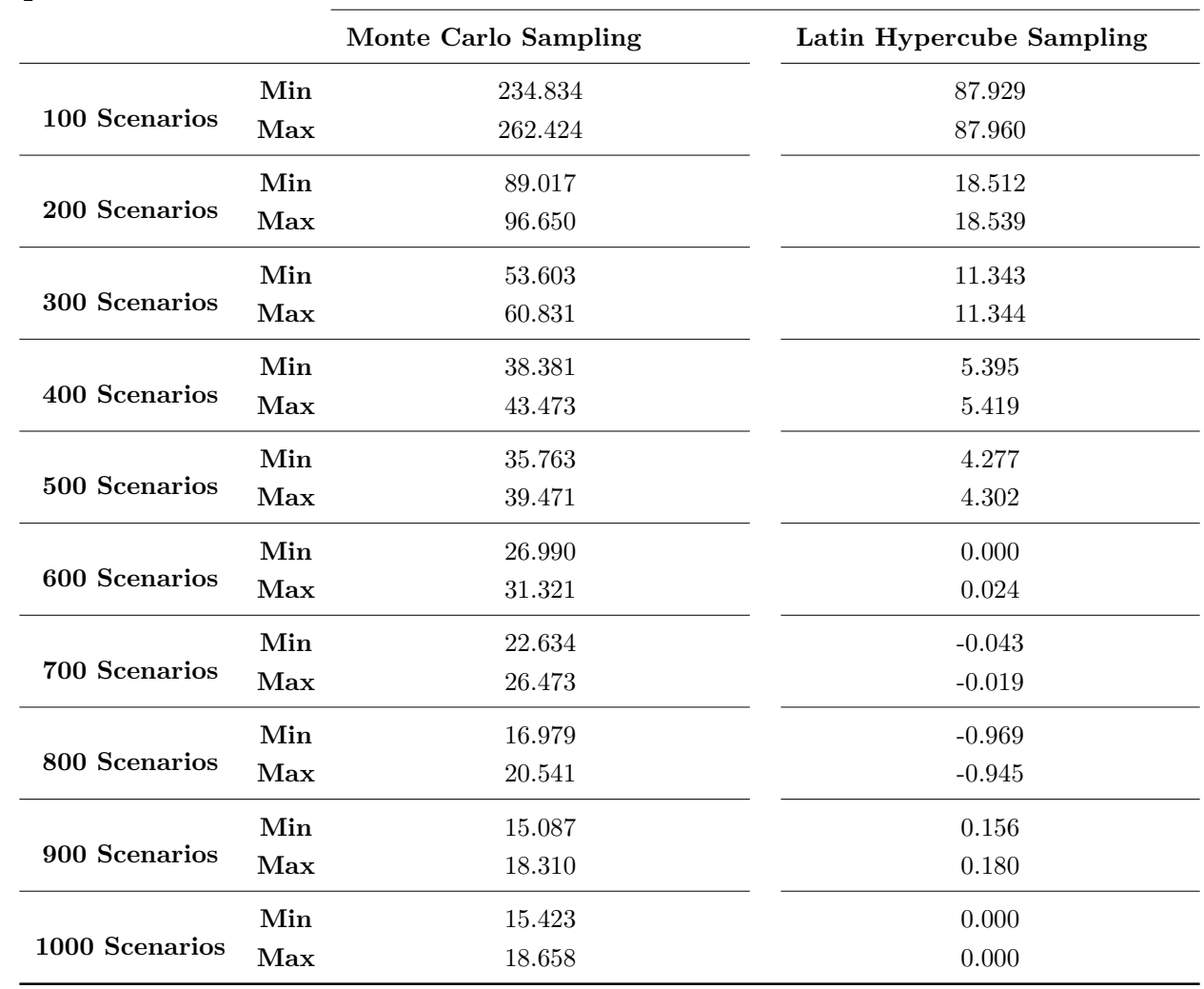

Tables F.2 and F.3 show the number of first-stage solutions in the insample analysis, i.e., how many different solutions were observed in each replication. In the tables, we present the results of each replication by varying the number of scenarios using the Monte Carlo and the Latin Hypercube methods, respectively. The solution we consider for analysis here is the number of physicians allocated for each day and shift. For example, in Table F.2 we see that using 100 scenarios we have 5 possible solutions for the number of physicians that should be allocated on Monday's Shift 1.

We can observe from Table F.2 that as we increase the number of scenarios, the number of possible solutions decreases, tending to converge to a 
single solution. We see that the Average Number of Solutions (ANS) is 3.8 for 100 scenarios and decreases progressively reaching 2.3 for 1,000 scenarios. We observed the same behavior in Table F.3 using the Latin Hypercube technique. However, we noticed a faster convergence tending to a single solution using this sampling method (ANS $=1.5$ for 1,000 scenarios).

Table F.2: Number of solutions using Monte Carlo Sampling method

\begin{tabular}{|c|c|c|c|c|c|c|c|c|c|c|c|c|c|c|c|c|c|c|c|c|c|c|c|c|c|c|c|c|c|c|c|c|c|c|c|c|c|c|c|c|}
\hline \multirow{2}{*}{\multicolumn{2}{|c|}{ Shifts $^{a}$}} & \multicolumn{5}{|c|}{ Monday } & \multicolumn{6}{|c|}{ Tuesday } & \multicolumn{5}{|c|}{ Wednesday } & \multicolumn{6}{|c|}{ Thursday } & \multicolumn{5}{|c|}{ Friday } & \multicolumn{5}{|c|}{ Saturday } & \multicolumn{6}{|c|}{ Sunday } & \multirow[b]{2}{*}{$\mathrm{ANS}^{\mathrm{b}}$} \\
\hline & & & & & & 67 & 1 & 12 & & & 6 & & 1 & & & & 7 & & & & 5 & 6 & & 12 & & 7 & & 7 & 1 & & 34 & & 67 & & 12 & 34 & 45 & & & \\
\hline & 100 & 5 & & & 2 & & 3 & & & & 4 & & 4 & & 5 & 3 & & 4 & & 45 & & 4 & & 44 & & 5 & & & 3 & & & 4 & & & & 45 & $\begin{array}{ll}53 \\
\end{array}$ & & & 3.8 \\
\hline & 200 & 3 & 3 & 35 & 4 & $\begin{array}{l}32 \\
\end{array}$ & 3 & 34 & 34 & 3 & 3 & 2 & 2 & 33 & 3 & $3:$ & 2 & 3 & & 34 & 3 & 2 & & 34 & 43 & 4 & $\begin{array}{ll}33 \\
\end{array}$ & 2 & 3 & 3 & $\begin{array}{ll}34 \\
\end{array}$ & 3 & 32 & & 44 & 34 & 43 & 2 & & .0 \\
\hline & 300 & 3 & 3 & 34 & 3 & 22 & 3 & 3 & 35 & 3 & 2 & 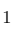 & 2 & 23 & 3 & 3 & 2 & 3 & 3 & 3 & 2 & 2 & 2 & 33 & 33 & 4 & 33 & 2 & 3 & 3 & 34 & 3 & 32 & & 33 & 23 & 33 & 3 & & 2.8 \\
\hline & 400 & 3 & 3 & 34 & 3 & 22 & 2 & 24 & 35 & 3 & 3 & & 2 & $\begin{array}{ll}33 \\
\end{array}$ & 3 & 3 & 1 & 3 & 3 & 33 & 2 & 2 & 2 & 22 & 23 & 4 & 23 & 2 & 3 & 4 & 23 & 3 & 32 & & $\begin{array}{l}34 \\
\end{array}$ & 23 & $\begin{array}{ll}3 & 3\end{array}$ & 3 & & 2.8 \\
\hline & 500 & 3 & 3 & 23 & 3 & $\begin{array}{l}32 \\
\end{array}$ & 2 & 23 & 34 & 3 & 2 & & 2 & 23 & 3 & 2 & 1 & 3 & 3 & 24 & 3 & 2 & & 22 & 23 & 4 & 23 & 2 & 2 & 3 & $\begin{array}{ll}23 \\
\end{array}$ & 3 & 32 & & 23 & 34 & 43 & 3 & & 2.6 \\
\hline & 600 & 3 & & 34 & 3 & 22 & 3 & 33 & 34 & 3 & 2 & & 2 & 23 & 3 & 2 & & 3 & 2 & 23 & 2 & 3 & & & 23 & 4 & $\begin{array}{ll}33 \\
\end{array}$ & 2 & 2 & 3 & 23 & 3 & 32 & & & 23 & $\begin{array}{l}33 \\
\end{array}$ & 3 & & 2.6 \\
\hline & 700 & 2 & & 44 & 3 & 22 & 3 & $\begin{array}{ll}33 \\
\end{array}$ & 34 & 3 & 2 & & 2 & 22 & 3 & 2 & 1 & 2 & 3 & 23 & 2 & 2 & & $\begin{array}{ll}32 \\
\end{array}$ & 23 & 2 & 23 & 2 & 2 & 3 & 23 & 3 & 32 & & & 23 & & 3 & 2 & 2.5 \\
\hline & 800 & 3 & 3 & 33 & 3 & 22 & 2 & 22 & 33 & 3 & 2 & & 2 & 23 & 3 & 2 & 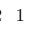 & 2 & & 23 & 2 & 1 & & 22 & & 2 & & 2 & 3 & 3 & $\begin{array}{lll}3 & 4\end{array}$ & 3 & 32 & & & 23 & & 2 & & 4 \\
\hline & 900 & 2 & 3 & 23 & 2 & 22 & 2 & 22 & 33 & & 2 & & 2 & 23 & & 2 & & 3 & & 23 & 2 & 2 & & 22 & & 3 & & 2 & 2 & 2 & & 3 & 32 & & $\begin{array}{ll}22 \\
\end{array}$ & 23 & $\begin{array}{ll}32 \\
\end{array}$ & 3 & 2 & 2.3 \\
\hline & 1000 & 2 & & 23 & & 22 & 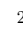 & 22 & 33 & & & & 2 & & & 2 & & 2 & & 23 & 2 & 2 & & 20 & & 3 & 22 & 2 & 2 & 3 & $\begin{array}{ll}33 \\
3\end{array}$ & 2 & 32 & & 23 & 23 & & 2 & 2 & 2.3 \\
\hline
\end{tabular}

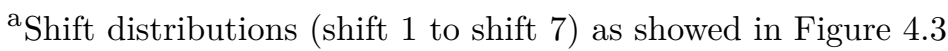

${ }^{\mathrm{b}}$ ANS - Average Number of Solutions

Table F.3: Number of solutions using Latin Hypercube Sampling method

\begin{tabular}{|c|c|c|c|c|c|c|c|c|c|c|c|c|c|c|c|c|c|c|c|c|c|c|c|c|c|c|c|c|c|c|c|c|c|c|c|c|c|c|}
\hline & & \multicolumn{5}{|c|}{ Monday } & \multicolumn{6}{|c|}{ Tuesday } & \multicolumn{5}{|c|}{ Wednesday } & \multicolumn{5}{|c|}{ Thursday } & \multicolumn{6}{|c|}{ Friday } & \multicolumn{5}{|c|}{ Saturday } & \multicolumn{4}{|c|}{ Sunday } & \multirow{2}{*}{$\mathrm{ANS}^{\mathrm{b}}$} \\
\hline & Shifts ${ }^{a}$ & 1 & & & & 67 & & & & & 7 & & 1 & & & & & & 2 & 7 & 56 & 7 & & 2 & 4 & & 67 & & 12 & 8 & & 67 & & 12 & 34 & 156 & 7 & \\
\hline & 100 & 3 & & $\begin{array}{ll}35 \\
5\end{array}$ & 3 & 22 & & & 3 & 44 & 3 & & 3 & 44 & 43 & $\begin{array}{ll}34 \\
\end{array}$ & & & 4 & 44 & $\begin{array}{ll}32 \\
\end{array}$ & 8 & & 3 & $\begin{array}{ll}34 \\
\end{array}$ & 3 & $\begin{array}{ll}42 \\
4\end{array}$ & & $\begin{array}{ll}34 \\
\end{array}$ & 3 & 43 & 32 & & 44 & 34 & 33 & $\begin{array}{ll}32 \\
\end{array}$ & 3.2 \\
\hline & 200 & 3 & & 34 & & $\begin{array}{ll}32 \\
\end{array}$ & & & 3 & 32 & 2 & & 2 & . & 42 & 23 & 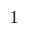 & & 2 & $\begin{array}{ll}23 \\
\text {. }\end{array}$ & 22 & 1 & & 3 & 34 & & 3 & & 23 & 33 & 33 & & 2 & 33 & 33 & 22 & 22 & .6 \\
\hline & 300 & 3 & & 24 & 3 & 22 & & 2 & 3 & 43 & 2 & & 2 & 22 & 32 & 22 & 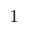 & & 2 & 23 & $\begin{array}{ll}21 \\
\end{array}$ & 1 & & 2 & $\begin{array}{ll}33 & 3\end{array}$ & 2 & 3 & & $\begin{array}{ll}33 \\
3\end{array}$ & $\begin{array}{l}33 \\
3\end{array}$ & 33 & 32 & & 22 & 23 & 22 & 22 & 14 \\
\hline & 400 & 2 & & $\begin{array}{l}34 \\
\end{array}$ & 3 & $\begin{array}{ll}22 \\
\end{array}$ & & & 3 & $\begin{array}{ll}32 \\
\end{array}$ & 2 & & 1 & 12 & 22 & $\begin{array}{ll}22 \\
\end{array}$ & 1 & 1 & 2 & $\begin{array}{ll}23 \\
\text { : }\end{array}$ & $\begin{array}{ll}21 \\
\end{array}$ & 11 & 2 & 2 & $\begin{array}{ll}33 \\
\end{array}$ & 2 & 22 & & 23 & 332 & 23 & 32 & & 22 & 23 & 22 & 22 & 1.1 \\
\hline & 500 & 3 & 3 & 23 & 3 & 22 & & & 3 & 42 & 2 & & 2 & 22 & 32 & 22 & 1 & 1 & 2 & 23 & $\begin{array}{ll}2 & 1\end{array}$ & 11 & 2 & 3 & $\begin{array}{ll}32 \\
\end{array}$ & & 22 & & $\begin{array}{ll}23 \\
\end{array}$ & $\begin{array}{ll}32 & 2\end{array}$ & 23 & & & 22 & & 21 & $\begin{array}{lll}11 & 1\end{array}$ & 2 \\
\hline & 600 & 2 & 2 & 22 & 2 & $\begin{array}{ll}22 & 2\end{array}$ & & & 3 & 32 & 2 & & 1 & 12 & 21 & 11 & 1 & 1 & 1 & $\begin{array}{ll}23 \\
\end{array}$ & 21 & 1 & 1 & 1 & 22 & 2 & 22 & & 22 & 222 & 23 & 22 & 2 & 22 & 32 & 22 & 21 & .8 \\
\hline & 700 & 2 & & $\begin{array}{ll}32 \\
\end{array}$ & 2 & 22 & & & 3 & 22 & & 1 & 1 & 22 & 11 & 11 & 1 & & 2 & $\begin{array}{ll}32 \\
3\end{array}$ & $\begin{array}{ll}1 & 1\end{array}$ & 1 & & 2 & $\begin{array}{ll}2 & 1\end{array}$ & 1 & 12 & & 22 & 22 & 22 & 12 & 2 & 22 & 32 & 11 & $\begin{array}{ll}11 & 1\end{array}$ & .7 \\
\hline & 800 & 1 & & 22 & 1 & 22 & & & 2 & 22 & 2 & 1 & 1 & $\begin{array}{ll}11 & 1\end{array}$ & 11 & 11 & 1 & & 1 & 11 & $\begin{array}{lll}1 & 1\end{array}$ & 11 & & 1 & 11 & 1 & 1 & & $\begin{array}{ll}12 \\
\end{array}$ & 221 & 12 & 21 & & 22 & 22 & 11 & $\begin{array}{lll}11 & \end{array}$ & \\
\hline & & 1 & & 22 & 1 & $\begin{array}{ll}22 \\
\end{array}$ & & & 2 & $\begin{array}{ll}32 \\
\end{array}$ & 2 & & 1 & 12 & 21 & 11 & 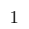 & & 1 & 22 & 21 & 11 & & 1 & 22 & 1 & 11 & & 12 & 221 & 12 & 21 & & & 22 & 11 & 1 & 1.5 \\
\hline & 1000 & 1 & & 23 & & & & & 3 & $\begin{array}{ll}32 \\
\end{array}$ & & & 1 & 12 & 21 & 11 & 1 & & 1 & 22 & $\begin{array}{ll}1 & 1\end{array}$ & 11 & 1 & 1 & 12 & & 11 & & & 221 & 12 & 21 & & 11 & 22 & 12 & 22 & 1.5 \\
\hline
\end{tabular}

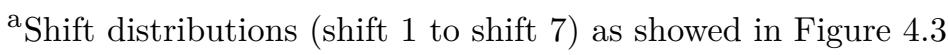

${ }^{\mathrm{b}}$ ANS - Average Number of Solutions

As for each number of scenarios, we run 50 replications, it is important to analyze the frequency with which each solution appears. With this, we can analyze dominant solutions. We call dominant solutions the solutions that appear most often. Tables F.4 - F.13 present the results of the dominant solution analysis for the Monte Carlo method. Tables F.14 - F.23 show the results for the Latin Hypercube technique. For dominant solution analysis, we use a color scale according to Figure F.1. When a solution appears 26 times or more (more than $50 \%$ ) we consider only 1 dominant solution, otherwise we consider as dominant solution any solution that appears between 11 and 25 times. 


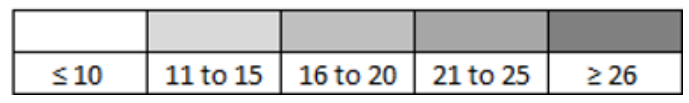

Figure F.1: Color scale used in the dominant solution analysis

Considering only the dominant solutions, we observed that even with just 100 scenarios we have a low number of possible solutions using both methods. Despite a large number of possible solutions, we see that as we increase the number of scenarios, even if other solutions ever appear, the solution tends to converge to a point, which we call the dominant solution and which can be considered the true solution for the problem. The results showed that the LHS method converges more quickly to a true solution since the ANS always decreases and from 300 scenarios the ANS is already 1.0 and remain the same in the tests with more scenarios. Using the MCS, we observed a greater oscillation of the ANS.

Table F.4: Analysis of dominant solution from Monte Carlo Sampling method using 100 scenarios

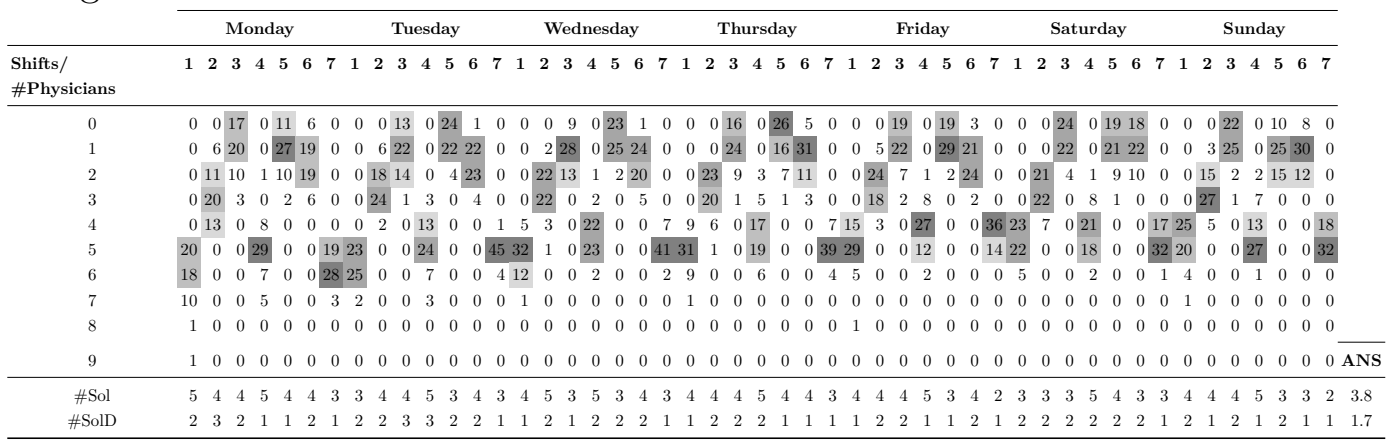

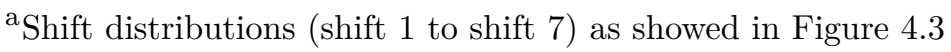

b \#Physicians - Number of Physicians assigned to that shift

${ }^{\mathrm{c}}$ \#Sol - Number of different solutions

$\mathrm{d}$ \#SolD - Number of dominant solutions

e ANS - Average Number of Solutions 
Table F.5: Analysis of dominant solution from Monte Carlo Sampling method using 200 scenarios

\begin{tabular}{|c|c|c|c|c|c|c|c|c|c|c|c|c|c|c|c|c|c|c|c|c|c|c|c|c|c|c|c|c|c|c|c|c|c|c|c|c|c|}
\hline \multirow[b]{2}{*}{$\begin{array}{l}\text { Shifts/ } \\
\text { \#Physicians }\end{array}$} & \multicolumn{5}{|c|}{ Monday } & \multicolumn{6}{|c|}{ Tuesday } & \multicolumn{5}{|c|}{ Wednesday } & \multicolumn{5}{|c|}{ Thursday } & \multicolumn{4}{|c|}{ Friday } & \multicolumn{6}{|c|}{ Saturday } & \multicolumn{5}{|c|}{ Sunday } & \\
\hline & $\begin{array}{ll}12 \\
\end{array}$ & & 4 & 5 & 7 & & 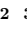 & 34 & & 6 & 71 & 2 & $\begin{array}{ll}3 & 4\end{array}$ & 5 & & & 2 & & & & & 2 & 3 & & & 71 & 12 & & & 56 & 7 & 1 & 2 & 34 & & & \\
\hline 0 & & 015 & 01 & 12 & 0 & & 01 & 60 & 19 & 0 & $\begin{array}{lll}0 & 0\end{array}$ & 0 & 170 & 027 & 2 & 0 & $\begin{array}{lll}0 & 0\end{array}$ & 11 & 015 & 0 & $\begin{array}{lll}0 & 0\end{array}$ & $\begin{array}{ll}0 & 0\end{array}$ & 14 & 025 & & & & 27 & $\begin{array}{lll}0 & 1\end{array}$ & 1419 & & 0 & 02 & 220 & 011 & & \\
\hline 1 & & 028 & 302 & 272 & & & 32 & & 26 & & & 2 & $\begin{array}{ll}30 & 0\end{array}$ & $022:$ & & & $\begin{array}{ll}0 & 0\end{array}$ & 35 & 0314 & & $\begin{array}{lll}0 & 0\end{array}$ & & 25 & 022 & 21 & & & 21 & & 3221 & & & 32 & & 032 & & 0 \\
\hline 2 & 014 & 146 & 0 & 92 & 20 & 0 & & $\begin{array}{lll}9 & 0\end{array}$ & 5 & 30 & $\begin{array}{ll}0 \quad 0 \\
0\end{array}$ & 22 & 30 & $\begin{array}{lll}0 & 1\end{array}$ & & 0 & $\begin{array}{lll}0 & 16\end{array}$ & 40 & 04 & & $\begin{array}{lll}0 & 0\end{array}$ & & 10 & & 26 & 0 & 013 & 31 & & 49 & 90 & & & & & & 0 \\
\hline 3 & 023 & $\begin{array}{ll}23 & 0\end{array}$ & 0 & 1 & 20 & 0 & & $\begin{array}{lll}0 & 1\end{array}$ & 0 & 1 & & 26 & $\begin{array}{lll}0 & 0\end{array}$ & $\begin{array}{lll}0 & 0\end{array}$ & 0 & 0 & 030 & 0 & $\begin{array}{lll}4 & 0\end{array}$ & 0 & & 016 & 0 & 70 & 0 & 0 & 033 & & 2 & & & & & $\begin{array}{lll}0 & 3\end{array}$ & $\begin{array}{lll}3 & 0\end{array}$ & & 0 \\
\hline 4 & 013 & & 5 & & & 0 & & & & 0 & & 0 & 014 & & 0 & 0 & $\begin{array}{lll}9 & 4\end{array}$ & 028 & & 0 & & $\begin{array}{lll}9 & 1\end{array}$ & 02 & & & 3621 & 214 & & 25 & & & & & 020 & $\begin{array}{lll}0 & 0\end{array}$ & 01 & \\
\hline 5 & 180 & & 25 & & 18 & & 0 & 026 & & & 734 & & 030 & & & 4739 & & 017 & & 04 & 1837 & 70 & 01 & 130 & & 1426 & 260 & & 21 & & 30 & & & 024 & $\begin{array}{ll}40 \\
0\end{array}$ & 03 & \\
\hline 6 & 270 & & 15 & & 32 & & 0 & 014 & 0 & & 316 & 0 & 06 & $\begin{array}{lll}6 & 0\end{array}$ & & 3 & 20 & 0 & $\begin{array}{lll}1 & 0\end{array}$ & & & 40 & & 30 & & 03 & 30 & & 2 & & & & & 0 & $\begin{array}{ll}3 & 0\end{array}$ & & 0 \\
\hline 7 & & $\begin{array}{lll}0 & 0\end{array}$ & & 0 & & & 0 & $\begin{array}{lll}0 & 0\end{array}$ & 0 & & & 0 & $\begin{array}{ll}0 & 0\end{array}$ & $\begin{array}{lll}0 & 0\end{array}$ & & 0 & $\begin{array}{lll}0 & 0\end{array}$ & 0 & $\begin{array}{lll}0 & 0\end{array}$ & 0 & & $\begin{array}{lll}0 & 0\end{array}$ & 0 & $\begin{array}{lll}0 & 0\end{array}$ & 0 & $\begin{array}{lll}0 & 0\end{array}$ & $\begin{array}{ll}0 & 0\end{array}$ & $\begin{array}{ll}0 & 0\end{array}$ & 0 & $\begin{array}{lll}0 & 0\end{array}$ & $\begin{array}{lll}0 & 0\end{array}$ & 1 & & 0 & $\begin{array}{lll}0 & 0\end{array}$ & & \\
\hline 8 & $\begin{array}{lll}0 & 0\end{array}$ & $\begin{array}{ll}0 & 0\end{array}$ & 1 & 0 & & 0 & 0 & $\begin{array}{lll}0 & 0\end{array}$ & 0 & 0 & $\begin{array}{lll}0 & 0\end{array}$ & 0 & $\begin{array}{ll}0 & 0\end{array}$ & $\begin{array}{lll}0 & 0\end{array}$ & 0 & 0 & $\begin{array}{lll}0 & 0\end{array}$ & 0 & $\begin{array}{lll}0 & 0\end{array}$ & 0 & 00 & $\begin{array}{ll}0 & 0\end{array}$ & 0 & $\begin{array}{ll}0 & 0\end{array}$ & 0 & $\begin{array}{lll}0 & 0\end{array}$ & $\begin{array}{ll}0 & 0\end{array}$ & $\begin{array}{ll}0 & 0\end{array}$ & 0 & $\begin{array}{ll}0 & 0\end{array}$ & $\begin{array}{ll}0 & 0\end{array}$ & 0 & 0 & 0 & $\begin{array}{lll}0 & 0\end{array}$ & 0 & $0 \overline{\mathrm{AN}}$ \\
\hline & & & 5 & & & & & & 3 & & & 3 & $\begin{array}{ll}3 & 3\end{array}$ & & 3 & & $\begin{array}{ll}3 & 3\end{array}$ & & & 2 & & & & & & 23 & & & & & & & & 3 & 4 & & \\
\hline 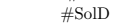 & 1 & 21 & 2 & & & & 1 & 21 & 1 & 1 & & 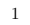 & 11 & 11 & 1 & 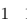 & 11 & 1 & 11 & 1 & 1 & 11 & 2 & 12 & 1 & 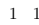 & 11 & 11 & 2 & 12 & 21 & 1 & 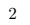 & 12 & 21 & 1 & $\begin{array}{ll}1 & 1.2\end{array}$ \\
\hline
\end{tabular}

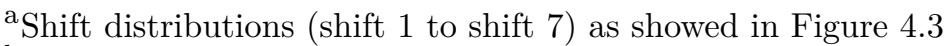

${ }^{\mathrm{b}}$ \#Physicians - Number of Physicians assigned to that shift

${ }^{\mathrm{c}}$ \#Sol - Number of different solutions

d \#SolD - Number of dominant solutions

${ }^{\mathrm{e}}$ ANS - Average Number of Solutions

Table F.6: Analysis of dominant solution from Monte Carlo Sampling method using 300 scenarios

\begin{tabular}{|c|c|c|c|c|c|c|c|c|c|c|c|c|c|c|c|c|c|c|c|c|c|c|c|c|c|c|c|c|c|c|c|c|c|c|c|c|c|c|}
\hline \multirow[b]{2}{*}{$\begin{array}{l}\text { Shifts/ } \\
\text { \#Physicians }\end{array}$} & \multicolumn{6}{|c|}{ Monday } & \multicolumn{5}{|c|}{ Tuesday } & \multicolumn{5}{|c|}{ Wednesday } & \multicolumn{6}{|c|}{ Thursday } & \multicolumn{5}{|c|}{ Friday } & \multicolumn{6}{|c|}{ Saturday } & \multicolumn{4}{|c|}{ Sunday } & \\
\hline & 12 & $\begin{array}{ll}2 & 3 \\
\end{array}$ & 34 & & $\begin{array}{ll}6 & 7\end{array}$ & 71 & & & & & 7 & & & & & & & & & & & & & 23 & & & $\begin{array}{ll}67 \\
6\end{array}$ & 1 & 2 & 34 & & 67 & 71 & 12 & 234 & 45 & & \\
\hline 0 & & 018 & $\begin{array}{lll}8 & 01 \\
\end{array}$ & 12 & 00 & $\begin{array}{ll}0 & 0\end{array}$ & 00 & 8 & 019 & & 0 & & $\begin{array}{lll}0 & 19 \\
\end{array}$ & 02 & 22 & & & 0 & & & & & & 015 & & 36 & & & & $\begin{array}{ll}30 & 0 \\
\end{array}$ & & & & & 021 & 022 & & \\
\hline 1 & & 026 & & 303 & & & & 33 & 027 & & 0 & & $\begin{array}{l}022 \\
022\end{array}$ & & 263 & & & 0 & & 028 & 44 & & & 232 & & 122 & & & & $\begin{array}{lll}19 & 0 \\
19\end{array}$ & 029 & & & & $\begin{array}{l}129 \\
129\end{array}$ & 027 : & $\begin{array}{ll}32 & 0\end{array}$ & \\
\hline 2 & & 11 & & 81 & & & 26 & 8 & 04 & 33 & & 02 & 39 & 0 & 11 & & & 15 & 3 & 0 & 6 & 0 & 03 & & 30 & 12 ? & & & 22 & & $\begin{array}{ll}0 & 3 \\
0 & 3\end{array}$ & 5 & & 034 & $\begin{array}{llll}4 & 25 & 0 \\
4 & 0\end{array}$ & & $\begin{array}{ll}11 & 0 \\
11 & 0\end{array}$ & 0 \\
\hline 3 & & 35 & & 0 & & & $\begin{array}{l}20 \\
23\end{array}$ & $\begin{array}{l}8 \\
0\end{array}$ & & 0 & & 02 & & 0 & $\begin{array}{l}1 \\
0\end{array}$ & & & 32 & 0 & & & & 01 & & 3 & & & & 22 & & & & & $\begin{array}{l}015 \\
015\end{array}$ & & & & 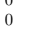 \\
\hline 4 & & & $\begin{array}{lll}0 & 4\end{array}$ & & & $\begin{array}{ll}0 & 0\end{array}$ & 0 & 01 & & & 0 & 0 & & 21 & & & 011 & 3 & 01 & & & 1 & & & 24 & & & 422 & 6 & 014 & & & $\begin{array}{ll}11 & 9\end{array}$ & $\begin{array}{ll}9 & 0\end{array}$ & $\begin{array}{llll}0 & 0 & 1\end{array}$ & 150 & 014 & \\
\hline 5 & 11 & 0 & 016 & & & 122 & 20 & 02 & & & $50:$ & & $\begin{array}{ll}0 & 0\end{array}$ & 24 & & & 935 & 0 & 02 & & & 49 & 36 & $\begin{array}{ll}0 & 0\end{array}$ & 21 & 0 & & 627 & 0 & 029 & & & 3940 & & $\begin{array}{lll}0 & 0 & 2\end{array}$ & $\begin{array}{ll}23 & 0\end{array}$ & 036 & \\
\hline 6 & 35 & 0 & 027 & 0 & & 927 & 70 & 0 & 80 & & 0 & & $\begin{array}{ll}0 & 0\end{array}$ & 5 & 0 & & 1 & 10 & 0 & 8 & 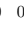 & & 4 & 0 & 2 & 0 & & 1 & 0 & $\begin{array}{ll}03 \\
3\end{array}$ & 0 & & $\begin{array}{lll}0 & 1\end{array}$ & 0 & $\begin{array}{lll}0 & 0 & 1\end{array}$ & 120 & & \\
\hline 7 & 4 & 0 & $\begin{array}{lll}0 & 3 \\
\end{array}$ & 0 & 0 & $\begin{array}{ll}0 & 1\end{array}$ & 10 & 0 & 10 & 0 & 0 & 0 & $\begin{array}{ll}0 & 0\end{array}$ & 0 & 0 & 0 & $\begin{array}{ll}0 & 0\end{array}$ & 0 & 0 & 0 & 0 & 0 & 0 & $\begin{array}{ll}0 & 0\end{array}$ & 00 & 0 & 0 & $\begin{array}{ll}0 & 0\end{array}$ & 0 & $\begin{array}{ll}0 & 0\end{array}$ & $\begin{array}{ll}0 & 0\end{array}$ & 0 & $\begin{array}{ll}0 & 0\end{array}$ & $\begin{array}{ll}0 & 0\end{array}$ & $\begin{array}{lll}0 & 0\end{array}$ & $\begin{array}{ll}0 & 0\end{array}$ & ) 00 & $0 \overline{\mathrm{ANS}}$ \\
\hline & 3 & 3 & 34 & 3 & 2 & 23 & 33 & 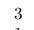 & $\begin{array}{ll}5 & 3 \\
\end{array}$ & 2 & 1 & 2 & 23 & 3 & 3 & 22 & 23 & 33 & 3 & 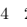 & 2 & 2 & 3 & $\begin{array}{ll}3 & 3 \\
\end{array}$ & 34 & 3 & 3 & 23 & 3 & 3 & 43 & 3 & 23 & $\begin{array}{ll}3 & 3\end{array}$ & 32 & $\begin{array}{ll}3 & 3\end{array}$ & 332 & \begin{tabular}{|l|l|}
2 & 2.8 \\
\end{tabular} \\
\hline & & & & & & & & & & & & & & & & & & & & & & & & & & & & & & & & & & & & & & \\
\hline
\end{tabular}

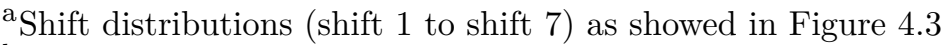

b \#Physicians - Number of Physicians assigned to that shift

${ }^{\mathrm{c}}$ \#Sol - Number of different solutions

d \#SolD - Number of dominant solutions

${ }^{\mathrm{e}} \mathrm{ANS}$ - Average Number of Solutions

Table F.7: Analysis of dominant solution from Monte Carlo Sampling method using 400 scenarios

\begin{tabular}{|c|c|c|c|c|c|c|c|c|c|c|c|c|c|c|c|c|c|c|c|c|c|c|c|c|c|c|c|c|c|c|c|c|c|c|c|c|c|}
\hline \multirow[b]{2}{*}{$\begin{array}{l}\text { Shifts/ } \\
\text { \#Physicians }\end{array}$} & \multicolumn{6}{|c|}{ Monday } & \multicolumn{5}{|c|}{ Tuesday } & \multicolumn{5}{|c|}{ Wednesday } & \multicolumn{5}{|c|}{ Thursday } & \multicolumn{5}{|c|}{ Friday } & \multicolumn{5}{|c|}{ Saturday } & \multicolumn{6}{|c|}{ Sunday } \\
\hline & 12 & 23 & 4 & & 6 & 1 & 2 & 34 & 45 & 6 & $7 \quad 1$ & 2 & 34 & 45 & & & 2 & 34 & 45 & & 7 & & & 4 & & & 1 & 12 & 34 & 45 & 56 & 712 & $\begin{array}{ll}23 \\
\end{array}$ & 45 & & & \\
\hline 0 & 00 & 025 & & 11 & & & & 12 & 021 & & 00 & 0 & 18 & 016 & 0 & 0 & 00 & 18 & 016 & 0 & 0 & 0 & 011 & 10 & 31 & & & & 27 & 017 & 717 & $\begin{array}{ll}0 & 0\end{array}$ & 021 & 1018 & 182 & 0 & \\
\hline 1 & & 023 & & 333 & & & & 31 & & & & & & $033:$ & & & & & 034 & & & & & & & & & & & & 226 & & 128 & & & & \\
\hline & & & & & & & & & & & & & 35 & & & & 012 & & & & & & & & 0 & & & & & & & & & & & 0 & \\
\hline 4 & $\begin{array}{ll}0 & 3.4 \\
0 & 9\end{array}$ & 340 & 0 & 0 & & 0 & 28 & 0 & 10 & 0 & 0 & 035 & 0 & $\begin{array}{lll}0 & 0 \\
0\end{array}$ & 0 & 0 & 037 & & $\begin{array}{ll}0 & 0 \\
0\end{array}$ & 0 & 0 & 0 & 9 & & 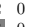 & & & 023 & $\begin{array}{ll}3 & 0 \\
1 & \end{array}$ & & & & ) 21 & $\begin{array}{lll}0 & 0 \\
0\end{array}$ & & 0 & \\
\hline 5 & 13 & & $\begin{array}{l}1 \\
15\end{array}$ & & & $\begin{array}{llll}0 & 0 & 0 \\
9 & 29 & & \end{array}$ & & 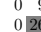 & & & $\begin{array}{ccc}0 & 1 \\
49 & 42 & 2\end{array}$ & & & $\begin{array}{lll}20 & 0 \\
27 & 0 & \end{array}$ & & 504 & $\begin{array}{ll}5 & 1 \\
4 & 0\end{array}$ & $\begin{array}{l}102 \\
0 \\
0\end{array}$ & & & $\begin{array}{r}2 \\
48\end{array}$ & & & 033 & 30 & & 513 & & $\begin{array}{lll}1 & 0 & 1 \\
0 & 0 & 3 \\
0\end{array}$ & & & $\begin{array}{l}1613 \\
3435\end{array}$ & $\begin{array}{lll}1 & 1 & 0 \\
3 & 0 & 0\end{array}$ & $\begin{array}{l}15 \\
150 \\
30\end{array}$ & 0 & 13 & \\
\hline 6 & 36 & $\begin{array}{lll}0 & 0 \\
\end{array}$ & 26 & 0 & 04 & $\begin{array}{l}3<y \\
121\end{array}$ & 10 & $\begin{array}{l}024 \\
01\end{array}$ & $\begin{array}{ll}3 & 0 \\
3 & 0\end{array}$ & & $\begin{array}{l}438 \\
18\end{array}$ & 80 & 0 & $\begin{array}{rr}3 & 0 \\
3 & 0\end{array}$ & & 0 & 10 & 0 & 30 & 0 & 0 & 0 & 000 & $\begin{array}{lll}0 & 14 \\
0\end{array}$ & 0 & & 0 & 20 & $\begin{array}{lll}0 & 0 \\
0\end{array}$ & 10 & $\begin{array}{llll}0 & 0\end{array}$ & 02 & $\begin{array}{ll}0 & 0\end{array}$ & $\begin{array}{l}5 \\
5\end{array}$ & $\begin{array}{lll}0 & 0\end{array}$ & 0 & \\
\hline 7 & 10 & $\begin{array}{ll}0 & 0\end{array}$ & 08 & 0 & 0 & $\begin{array}{ll}0 & 0\end{array}$ & $\begin{array}{ll}0 & 0\end{array}$ & 0 & 10 & & 00 & $\begin{array}{ll}0 & 0 \\
\end{array}$ & $\begin{array}{lll}0 & 0 & 1\end{array}$ & $\begin{array}{ll}0 & 0\end{array}$ & 0 & 0 & $\begin{array}{ll}0 & 0\end{array}$ & 00 & $\begin{array}{ll}0 & 0\end{array}$ & $\begin{array}{ll}0 & 0\end{array}$ & 0 & 0 & 00 & $\begin{array}{ll}0 & 0 \\
2\end{array}$ & 0 & 0 & 0 & $\begin{array}{ll}0 & 0\end{array}$ & $\begin{array}{lll}0 & 0\end{array}$ & 0 & $\begin{array}{ll}0 & 0 \\
\end{array}$ & $\begin{array}{ll}0 & 0\end{array}$ & $\begin{array}{ll}0 & 0\end{array}$ & $\begin{array}{ll}0 & 0\end{array}$ & $\begin{array}{ll}0 & 0\end{array}$ & & ANS \\
\hline$\# \mathrm{~S}$ & 3 & 3 & 34 & 3 & 2 & 2 & 24 & 3 & $\begin{array}{ll}5 & 3 \\
\end{array}$ & 3 & 2 & 23 & $\begin{array}{ll}33 \\
3\end{array}$ & $\begin{array}{ll}3 & 3\end{array}$ & 2 & 1 & $\begin{array}{l}33 \\
\end{array}$ & 33 & 32 & 22 & 2 & 2 & 23 & 34 & 2 & 3 & 2 & $\begin{array}{ll}34 \\
\end{array}$ & 42 & 3 & 3 & 2 & $\begin{array}{ll}44 & 2 \\
\end{array}$ & 233 & $\begin{array}{ll}3 & 3 \\
\end{array}$ & 2 & \\
\hline & & & & 1 & & & & & 1 & & & & 1 & $\begin{array}{lll}11 \\
\end{array}$ & & 1 & 1 & 1 & 1 & 1 & & & 1 & $\begin{array}{lll}1 & 1\end{array}$ & 1 & 1 & 1 & & 21 & 11 & & & 1 & 111 & 11 & 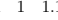 & 1 \\
\hline
\end{tabular}

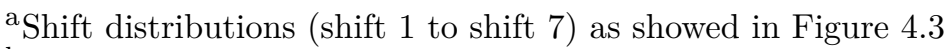

b \#Physicians - Number of Physicians assigned to that shift

${ }^{c}$ \#Sol - Number of different solutions

$\mathrm{d}$ \#SolD - Number of dominant solutions

${ }^{\mathrm{e}}$ ANS - Average Number of Solutions 
Table F.8: Analysis of dominant solution from Monte Carlo Sampling method using 500 scenarios

\begin{tabular}{|c|c|c|c|c|c|c|c|c|c|c|c|c|c|c|c|c|c|c|c|c|c|c|c|c|c|c|c|c|c|c|c|c|c|c|c|c|c|}
\hline \multirow[b]{2}{*}{$\begin{array}{l}\text { Shifts/ } \\
\text { \#Physicians }\end{array}$} & \multicolumn{5}{|c|}{ Monday } & \multicolumn{6}{|c|}{ Tuesday } & \multicolumn{5}{|c|}{ Wednesday } & \multicolumn{5}{|c|}{ Thursday } & \multicolumn{5}{|c|}{ Friday } & \multicolumn{5}{|c|}{ Saturday } & \multicolumn{5}{|c|}{ Sunday } & \\
\hline & 12 & & 34 & & 67 & & & 34 & 45 & & & & 34 & 5 & & & 12 & 3 & 45 & 6 & 7 & 12 & 23 & 4 & & & & 2 & 34 & 45 & 6 & 71 & 12 & 3 & 45 & 67 & \\
\hline 0 & & 02 & 80 & 9 & 0 & 0 & 0 & 16 & 024 & 0 & 0 & $\begin{array}{lll}0 & 0\end{array}$ & 20 & 026 & 0 & 0 & $\begin{array}{lll}0 & 0\end{array}$ & 023 & 016 & 0 & 0 & 0 & 014 & 403 & 35 & & & 0 & $24 \quad 0$ & 017 & & & & 26 & 017 & & \\
\hline 1 & & 02 & & 353 & & & & 29 & 025 & & & & 26 & 024 & 34 & & & 27 & 033 & & & & 030 & & 142 & & & & & 032 & & & & & 0313 & & \\
\hline 2 & & & $\begin{array}{lll}0 & 0\end{array}$ & 61 & & & 30 & 4 & & 35 & & $\begin{array}{lll}0 & 19\end{array}$ & 4 & $\begin{array}{lll}0 & 0\end{array}$ & 16 & & $\begin{array}{lll}0 & 10\end{array}$ & 0 & $\begin{array}{lll}0 & 1\end{array}$ & & 0 & 04 & & $\begin{array}{ll}50 \\
5\end{array}$ & 02 & & & 16 & & $\begin{array}{ll}0 & 1 \\
0\end{array}$ & & & 026 & & & 14 & \\
\hline 3 & & 29 & $\begin{array}{lll}0 & 0\end{array}$ & 0 & 1 & & 18 & 0 & & 0 & & $\begin{array}{lll}031 \\
0\end{array}$ & $\begin{array}{ll}4 \\
0\end{array}$ & $\begin{array}{lll}0 & 0\end{array}$ & & 0 & $\begin{array}{l}037 \\
0\end{array}$ & & & & & & & $\begin{array}{lll}0 & 0 \\
0 & 0\end{array}$ & & & & 31 & & & & & $\begin{array}{l}023 \\
023\end{array}$ & & & & \\
\hline 4 & & & $\begin{array}{lll}0 & 0\end{array}$ & 0 & & & 2 & & & & & $\begin{array}{lll}0 & 0\end{array}$ & 01 & & & 0 & 53 & & & & & & & 026 & & & 221 & & 019 & & & 1712 & & & & 01 & 15 \\
\hline 5 & 16 & & $\begin{array}{lll}0 & 14\end{array}$ & 0 & $\begin{array}{ll}01 \\
\end{array}$ & 220 & 0 & 02 & & & 504 & 110 & 03 & & & 504 & 40 & 0 & & & 504 & & & 021 & 0 & & 829 & 0 & 028 & & & 3338 & & & 250 & 03 & 35 \\
\hline 6 & 32 & & 032 & 0 & & 330 & 0 & 0 if & $\begin{array}{ll}6 & 0\end{array}$ & & 0 & $\begin{array}{ll}9 & 0\end{array}$ & 0 & & & & 10 & $\begin{array}{lll}0 & 0\end{array}$ & $\begin{array}{ll}50 \\
0\end{array}$ & & & & & & 0 & & 0 & 0 & & $\begin{array}{ll}30 \\
3\end{array}$ & & & 0 & & $\begin{array}{lll}9 & 0\end{array}$ & & \\
\hline 7 & 2 & 0 & $\begin{array}{lll}0 & 4\end{array}$ & 0 & 0 & 0 & 0 & 0 & $\begin{array}{ll}10 & 0\end{array}$ & 0 & 0 & $\begin{array}{ll}0 & 0\end{array}$ & 0 & $\begin{array}{ll}0 & 0\end{array}$ & 0 & 0 & $\begin{array}{ll}0 & 0\end{array}$ & 0 & $\begin{array}{ll}0 & 0\end{array}$ & 0 & 0 & 0 & $\begin{array}{lll}0 & 0\end{array}$ & $\begin{array}{lll}0 & 0\end{array}$ & 0 & 0 & 0 & 0 & $\begin{array}{ll}0 & 0\end{array}$ & $\begin{array}{ll}0 & 0\end{array}$ & 0 & $\begin{array}{ll}0 & 0\end{array}$ & $\begin{array}{ll}0 & 0\end{array}$ & 0 & $\begin{array}{ll}0 & 0\end{array}$ & (1) & $0 \overline{\mathrm{AN}}$ \\
\hline & 3 & & 23 & 3 & 3 & & & 3 & 43 & & & 22 & 3 & 32 & 2 & & & & 43 & 32 & & 2 & & 34 & 2 & & & & 2 & 3 & & & & & & & \\
\hline & . & 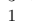 & $\begin{array}{ll}1 & 1\end{array}$ & 1 & 1 & 1 & & 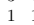 & 12 & & 1 & $\begin{array}{ll}1 & 1\end{array}$ & 1 & $\begin{array}{ll}1 & 1\end{array}$ & 1 & 1 & $\begin{array}{ll}1 & 1\end{array}$ & 11 & $\begin{array}{ll}1 & \end{array}$ & 1 & & 1 & 1 & $\begin{array}{lll}1 & 1\end{array}$ & 1 & 2 & 11 & 1 & 21 & 11 & 1 & 11 & 11 & 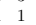 & 21 & & \\
\hline
\end{tabular}

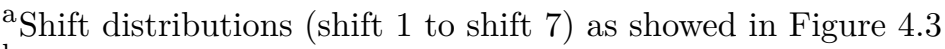

${ }^{\mathrm{b}}$ \#Physicians - Number of Physicians assigned to that shift

c \#Sol - Number of different solutions

d\#SolD - Number of dominant solutions

${ }^{\mathrm{e}}$ ANS - Average Number of Solutions

Table F.9: Analysis of dominant solution from Monte Carlo Sampling method using 600 scenarios

\begin{tabular}{|c|c|c|c|c|c|c|c|c|c|c|c|c|c|c|c|c|c|c|c|c|c|c|c|c|c|c|c|c|c|c|c|c|c|c|c|c|c|c|c|}
\hline \multirow[b]{2}{*}{$\begin{array}{l}\text { Shifts/ } \\
\text { \#Physicians }\end{array}$} & \multicolumn{6}{|c|}{ Monday } & \multicolumn{5}{|c|}{ Tuesday } & \multicolumn{5}{|c|}{ Wednesday } & & \multicolumn{5}{|c|}{ Thursday } & \multicolumn{5}{|c|}{ Friday } & \multicolumn{6}{|c|}{ Saturday } & \multicolumn{5}{|c|}{ Sunday } & \\
\hline & 12 & 23 & 34 & & 67 & 7 & & 3 & & & 7 & & & & & & 1 & 2 & & & 6 & 7 & 12 & 23 & 34 & & & 71 & 12 & 3 & 45 & 56 & 7 & 12 & 23 & 4 & 56 & 7 & \\
\hline 0 & & & $\begin{array}{ll}40 \\
\end{array}$ & 7 & $0 \quad 0$ & 00 & 0 & 12 & $02 t$ & 30 & 0 & 0 & 024 & 0 & 17 & & $\begin{array}{ll}0 & 0\end{array}$ & & 300 & 013 & & & 0 & 014 & $\begin{array}{ll}4 & 0 \\
\end{array}$ & 36 & & & & 26 & & 122 & & & 025 & 50 & 20 & & \\
\hline 1 & 0 & 025 & & 413 & & 00 & & 33 & 022 & 211 & & & 024 & & 323 & & $\begin{array}{ll}0 & 0\end{array}$ & & 20 & 037 & 747 & & & 033 & 30 & 13 & & 0 & & 24 & & 2727 & & & 025 & & $273 t$ & & \\
\hline 2 & & 6 & $\begin{array}{lll}1 & 0\end{array}$ & 21 & & & 28 & $\frac{\pi}{4}$ & 0 & 139 & & 01 & 52 & 0 & 01 & & $\begin{array}{lll}0 & 0 \\
0\end{array}$ & & 0 & $\begin{array}{lll}0 & 0 \\
0\end{array}$ & 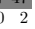 & 0 & 04 & & $\begin{array}{lll}3 & 0\end{array}$ & 15 & & 0 & & 0 & 01 & 11 & & 02 & & & 311 & & \\
\hline 3 & 03 & 38 & $\begin{array}{ll}1 & 0 \\
0 & 0\end{array}$ & 0 & & & 21 & 0 & $\begin{array}{l}0 \\
0\end{array}$ & 0 & & 03 & & 0 & 0 & & & 44 & & & 0 & & & & $\begin{array}{ll}0 & 0 \\
0 & 2\end{array}$ & 0 & & 0 & $\begin{array}{lll}0 & 17\end{array}$ & & & & & & & & & & \\
\hline 4 & & & $\begin{array}{ll}0 & 1\end{array}$ & 0 & & & 0 & 0 & & & 0 & 0 & & 10 & 0 & & $\begin{array}{lll}0 & 2 \\
\end{array}$ & 0 & 012 & & & 0 & & & 027 & & & 71 & & & 70 & & & 16 & & 013 & 0 & 011 & \\
\hline 5 & 11 & 0 & 020 & & & 521 & & 0 & & & 50 & & & 38 & 0 & & 047 & 70 & 03 & & & 49 & & & 018 & 0 & & 133 & & & & & 41 & 33 & & 032 & & 039 & \\
\hline 6 & 37 & & 026 & 0 & 03 & 528 & & 01 & 12 & & & & $\begin{array}{ll}0 & 0\end{array}$ & 2 & 0 & & & 0 & 0 & 40 & $\begin{array}{lll}0 & 0\end{array}$ & & 0 & 0 & $\begin{array}{ll}03 \\
3\end{array}$ & 0 & & 0 & $\begin{array}{ll}0 & 0\end{array}$ & 0 & . & 0 & & 1 & 0 & 5 & 0 & & \\
\hline 7 & 2 & $0 \quad 1$ & $\begin{array}{ll}0 \quad 3 \\
\end{array}$ & 0 & 0 & $\begin{array}{ll}0 \quad 1 \\
\end{array}$ & 0 & 0 & 2 & 00 & 0 & 0 & $\begin{array}{ll}0 & 0\end{array}$ & 0 & 0 & 00 & $\begin{array}{ll}0 & 0\end{array}$ & 0 & 00 & $\begin{array}{ll}0 & 0\end{array}$ & $\begin{array}{ll}0 & 0\end{array}$ & 0 & 0 & $0 \quad 0$ & $\begin{array}{ll}0 & 0\end{array}$ & 0 & 0 & 0 & $\begin{array}{ll}0 & 0\end{array}$ & 0 & 0 & $\begin{array}{ll}0 & 0\end{array}$ & 0 & 0 & $\begin{array}{ll}0 & 0\end{array}$ & $\begin{array}{ll}0 & 0\end{array}$ & 0 & $\begin{array}{ll}0 & 0\end{array}$ & $0 \widehat{\mathrm{ANS}}$ \\
\hline & & & & & & 2 & & 3 & & 32 & & & & 3 & 2 & & & & & & 23 & & & & 3 & 3 & & & & & 3 & 33 & & & & & 3 & & \\
\hline & 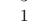 & & 2 & & & 2 & & 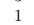 & 1 & . & 1 & 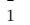 & $\begin{array}{ll}12 \\
2\end{array}$ & 1 & 1 & 1 & $\begin{array}{ll}1 & 1\end{array}$ & 1 & 11 & $\begin{array}{ll}1 & 1\end{array}$ & $\begin{array}{ll}1 & 1\end{array}$ & 1 & 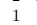 & 1 & $\begin{array}{ll}1 & 1\end{array}$ & . & 1 & 1 & $\begin{array}{ll}1 & 0\end{array}$ & 1 & 1 & 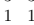 & & 1 & 2 & 21 & 1 & & $\begin{array}{ll}1 & 1.1\end{array}$ \\
\hline
\end{tabular}

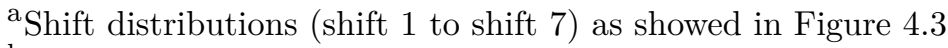

b \#Physicians - Number of Physicians assigned to that shift

c \#Sol - Number of different solutions

d \#SolD - Number of dominant solutions

${ }^{\mathrm{e}}$ ANS - Average Number of Solutions

Table F.10: Analysis of dominant solution from Monte Carlo Sampling method using 700 scenarios

\begin{tabular}{|c|c|c|c|c|c|c|c|c|c|c|c|c|c|c|c|c|c|c|c|c|c|c|c|c|c|c|c|c|c|c|c|c|c|c|c|c|}
\hline \multirow[b]{2}{*}{$\begin{array}{l}\text { Shifts/ } \\
\text { \#Physicians }\end{array}$} & \multicolumn{5}{|c|}{ Monday } & & \multicolumn{5}{|c|}{ Tuesday } & \multicolumn{4}{|c|}{ Wednesday } & \multicolumn{5}{|c|}{ Thursday } & \multicolumn{5}{|c|}{ Friday } & & \multicolumn{4}{|c|}{ Saturday } & \multicolumn{5}{|c|}{ Sunday } & \\
\hline & 12 & 23 & 45 & & 7 & & 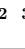 & $\begin{array}{ll}34 \\
4\end{array}$ & 5 & & 71 & 2 & 34 & 56 & 67 & & 23 & 34 & & 67 & & 2 & & & 6 & 71 & 12 & 34 & & & 71 & 2 & 34 & 56 & & \\
\hline 0 & & & & 90 & 0 & 0 & 0 & 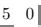 & 33 & 0 & 00 & 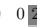 & 2901 & 13 & $\begin{array}{ll}0 & 0\end{array}$ & 0 & 02 & $\begin{array}{lll}27 & 0 \\
\end{array}$ & 15 & $\begin{array}{ll}0 & 0\end{array}$ & 0 & 01 & 15 & 042 & 2 & 0 & & 021 & 024 & 114 & $\begin{array}{lll}0 & 0\end{array}$ & 02 & 230 & 30 & & \\
\hline 1 & & 016 & $03 !$ & 3930 & 0 & & 23 & 370 & 11 & & & 02 & & 374 & & & & 220 & 344 & & & & & & & & & & 025 & & & & & 184 & & \\
\hline 2 & & & & & & 0 & & 70 & 6 & & & & $\begin{array}{ll}0 & 0 \\
0\end{array}$ & 0 & $\begin{array}{ll}9 & 0\end{array}$ & & & $\begin{array}{ll}0 & 0\end{array}$ & 0 & 10 & & 45 & & & 34 & & & & & & & & & & & 0 \\
\hline 3 & 03 & 391 & 0 & $\begin{array}{ll}0 & 0 \\
0\end{array}$ & 0 & 0 & & $\begin{array}{ll}0 & 0 \\
0\end{array}$ & 0 & 0 & $\begin{array}{lll}0 & 0 \\
0 & 0\end{array}$ & 40 & $\begin{array}{ll}0 & 0\end{array}$ & 0 & $\begin{array}{ll}0 & 0\end{array}$ & 0 & 39 & $\begin{array}{ll}0 & 0 \\
\end{array}$ & 0 & $\begin{array}{ll}0 & 0 \\
0\end{array}$ & 0 & 5 & 0 & $\begin{array}{ll}0 & 0 \\
0\end{array}$ & 0 & 0 & 021 & & $\begin{array}{ll}0 & 0 \\
\end{array}$ & & & 21 & $\begin{array}{ll}0 & 0\end{array}$ & & & 0 \\
\hline 4 & 0 & 50 & 2 & $\begin{array}{lll}0 & 0 \\
0 & 0 & 0\end{array}$ & 0 & & & $\begin{array}{ll}0 & 9 \\
0\end{array}$ & & 0 & $\begin{array}{lll}0 & 0 \\
0 & 0 & -15\end{array}$ & 0 & 010 & & $\begin{array}{lll}0 & 0 \\
0 & 0\end{array}$ & & 2 & 011 & & $\begin{array}{lll}0 & 0 \\
0 & 0 & 0\end{array}$ & & 0 & 02 & 60 & & 361 & & $\begin{array}{lll}1 & 01 \\
& 2\end{array}$ & 130 & & 1112 & 0 & 011 & 0 & & 9 \\
\hline 5 & & & 11 & & 19 & & & 023 & & & 1945 & 0 & 037 & & 050 & & 0 & 034 & & 050 & & 0 & 02 & & & 143 & 380 & $\begin{array}{lll}0 & 03\end{array}$ & & & 3937 & & 026 & 0 & & \\
\hline 6 & 43 & $\begin{array}{lll}0 & 03 \\
-1\end{array}$ & 32 & $\begin{array}{ll}0 & 0 \\
\end{array}$ & 31 & & 0 & 017 & & & 15 & 0 & $\begin{array}{lll}0 & 3\end{array}$ & 0 & $\begin{array}{lll}0 & 0\end{array}$ & 0 & 0 & 05 & 0 & & & 0 & 0 & $\begin{array}{ll}0 & 0\end{array}$ & & & $\begin{array}{ll}0 & 0\end{array}$ & & 20 & & & 0 & 013 & 0 & & \\
\hline 7 & 0 & $\begin{array}{ll}0 & 0 \\
\end{array}$ & 5 & $\begin{array}{ll}0 & 0 \\
\end{array}$ & 0 & 2 & 0 & $\begin{array}{ll}0 & 1 \\
\end{array}$ & 0 & 0 & $\begin{array}{ll}0 & 0 \\
\end{array}$ & 0 & $\begin{array}{ll}0 & 0 \\
\end{array}$ & 0 & $\begin{array}{ll}0 & 0 \\
\end{array}$ & 0 & 0 & $\begin{array}{ll}0 & 0 \\
\end{array}$ & 0 & $\begin{array}{ll}0 & 0 \\
\end{array}$ & 0 & 0 & 0 & $\begin{array}{ll}0 & 0 \\
\end{array}$ & 0 & 0 & $\begin{array}{ll}0 & 0 \\
\end{array}$ & $\begin{array}{ll}0 & 0 \\
\end{array}$ & $\begin{array}{ll}0 & 0 \\
\end{array}$ & 0 & $\begin{array}{lll}0 & 0 \\
\end{array}$ & 0 & $\begin{array}{ll}0 & 0 \\
\end{array}$ & 0 & 00 & 0 ANS \\
\hline$\#$ So & 2 & $\begin{array}{ll}34 \\
\end{array}$ & 4 & 3 & 22 & 3 & 3 & 34 & 3 & 2 & 22 & 2 & 23 & 2 & 21 & 2 & 3 & 23 & 2 & $\begin{array}{ll}2 & 1\end{array}$ & 3 & 2 & 3 & 22 & & 2 & 23 & $\begin{array}{ll}32 \\
\end{array}$ & $\begin{array}{ll}3 & 3 \\
\end{array}$ & 3 & $\begin{array}{ll}2 & 3\end{array}$ & 3 & 23 & 3 & 2 & 2 \\
\hline & & & & 1 & & & & 2 & & & & & $\begin{array}{lll}1 & 1\end{array}$ & 1 & 11 & & 1 & 11 & 1 & 1 & & 1 & 1 & $\begin{array}{lll}1 & 1\end{array}$ & & 1 & & 11 & 12 & & 1 & 1 & 11 & 1 & & \\
\hline
\end{tabular}

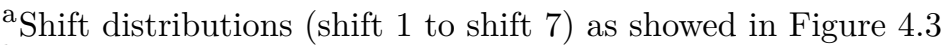

${ }^{\mathrm{b}}$ \#Physicians - Number of Physicians assigned to that shift

c \#Sol - Number of different solutions

$\mathrm{d}$ \#SolD - Number of dominant solutions

${ }^{\mathrm{e}} \mathrm{ANS}$ - Average Number of Solutions 
Table F.11: Analysis of dominant solution from Monte Carlo Sampling method using 800 scenarios

\begin{tabular}{|c|c|c|c|c|c|c|c|c|c|c|c|c|c|c|c|c|c|c|c|c|c|c|c|c|c|c|c|c|c|c|c|c|c|c|c|}
\hline \multirow[b]{2}{*}{$\begin{array}{l}\text { Shifts/ } \\
\text { \#Physicians }\end{array}$} & \multicolumn{6}{|c|}{ Monday } & \multicolumn{5}{|c|}{ Tuesday } & \multicolumn{4}{|c|}{ Wednesday } & \multicolumn{5}{|c|}{ Thursday } & \multicolumn{5}{|c|}{ Friday } & \multicolumn{5}{|c|}{ Saturday } & \multicolumn{4}{|c|}{ Sunday } & \\
\hline & & & & & & & 2 & 34 & & 67 & 71 & 2 & 4 & & & & & 34 & & & 1 & 23 & 34 & & & 1 & & 34 & $\begin{array}{ll}56 \\
\end{array}$ & 7 & 12 & 23 & 45 & & \\
\hline 0 & & 039 & 0 & & 0 & 0 & 01 & 140 & 26 & 0 & $\begin{array}{lll}0 & 0\end{array}$ & 05 & 310 & 16 & & & $0 \sqrt{3}$ & $\begin{array}{ll}30 & 0\end{array}$ & 60 & & & 010 & 100 & 400 & $\begin{array}{lll}0 & 0\end{array}$ & 0 & 02 & & 2416 & & & 020 & 029 & & \\
\hline 1 & & 010 & & 393 & & & 03 & & 22 & & & 01 & & 343 & & $\begin{array}{ll}0 & 0\end{array}$ & 02 & & 4350 & & & & 390 & 917 & & & 02 & & 2430 & & & & 020 & & \\
\hline 2 & & 51 & & 11 & & & 27 & 30 & 1 & 33 & & 13 & 10 & 01 & & & 2 & & 00 & 00 & 0 & 481 & 10 & 033 & & & 26 & 20 & 14 & & & & $\begin{array}{lll}0 & 0\end{array}$ & 11 & 0 \\
\hline$\sigma$ & 03. & & & & & & 23 & $\begin{array}{ll}0 & 0 \\
0\end{array}$ & 0 & 0 & & 37 & $\begin{array}{ll}0 & 0 \\
0\end{array}$ & 0 & & & 48 & & & & & 20 & & & $0 \quad 0$ & & 23 & & & 0 & 013 & & $\begin{array}{lll}0 & 0 \\
\end{array}$ & & \\
\hline 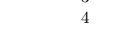 & 01 & & & & & & 0 & $\begin{array}{lll}0 & 2 \\
0 & \end{array}$ & & 0 & & 0 & $\begin{array}{ll}0 & 9\end{array}$ & 0 & & & 0 & $\begin{array}{lll}0 & 16 \\
\end{array}$ & & & & & 031 & & & & & & & 9 & & & & & \\
\hline 5 & 13 & $\begin{array}{ll}0 & 0\end{array}$ & & & & 123 & & 0 & & & 5043 & 0 & 034 & & & 047 & 0 & 031 & & 050 & & 00 & 019 & 0 & 011 & 36 & 0 & 036 & & 41 & 39 & & & 03 & 39 \\
\hline 6 & 35 & $\begin{array}{ll}0 & 0\end{array}$ & 36 & & $03 !$ & 27 & 0 & 016 & $\begin{array}{ll}6 & 0 \\
\end{array}$ & 0 & $\begin{array}{ll}0 & 7\end{array}$ & 70 & $\begin{array}{ll}0 & 7\end{array}$ & 0 & & $\begin{array}{lll}0 & 0\end{array}$ & 0 & 03 & 00 & $\begin{array}{ll}0 & 0 \\
\end{array}$ & 0 & 00 & $\begin{array}{lll}0 & 0\end{array}$ & 0 & & 1 & 0 & 03 & $\begin{array}{ll}0 & 0\end{array}$ & & & $\begin{array}{lll}0 & 0\end{array}$ & 120 & 0 & 0 \\
\hline 7 & 2 & $\begin{array}{ll}0 & 0\end{array}$ & 7 & 0 & 0 & 0 & 0 & 00 & $\begin{array}{ll}0 & 0\end{array}$ & 0 & $\begin{array}{ll}0 & 0\end{array}$ & 0 & $\begin{array}{ll}0 & 0\end{array}$ & 0 & $0 \mathrm{c}$ & $\begin{array}{ll}0 & 0\end{array}$ & 0 & $\begin{array}{ll}0 & 0\end{array}$ & 0 & $\begin{array}{ll}0 & 0\end{array}$ & 0 & 00 & $\begin{array}{ll}0 & 0\end{array}$ & 0 & $\begin{array}{ll}0 & 0\end{array}$ & 0 & 0 & $\begin{array}{lll}0 & 0\end{array}$ & $\begin{array}{ll}0 & 0\end{array}$ & $\begin{array}{ll}0 & 0\end{array}$ & 0 & $\begin{array}{ll}0 & 0\end{array}$ & $\begin{array}{ll}0 & 0\end{array}$ & 0 & $0 \overline{\mathrm{AN}}$ \\
\hline & . & $\begin{array}{ll}3 & 3\end{array}$ & 3 & 3 & 2 & 2 & 2 & $\begin{array}{ll}3 & 3 \\
\end{array}$ & 33 & 2 & $\begin{array}{ll}12 \\
12\end{array}$ & 22 & $\begin{array}{ll}3 & 3\end{array}$ & 2 & 21 & 12 & 2 & 23 & 2 & & 12 & & $\begin{array}{ll}32 \\
2\end{array}$ & 2 & 2 & 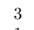 & & 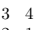 & $\begin{array}{ll}3 & 3\end{array}$ & & & 4 & $\begin{array}{ll}32 \\
3\end{array}$ & 2 & \\
\hline SolD & 1 & 1 & 1 & . & 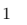 & & & 11 & 11 & 1 & 11 & 1 & $\begin{array}{ll}1 & 1\end{array}$ & 1 & 1 & 11 & 1 & $\begin{array}{ll}11 \\
1\end{array}$ & 1 & $\begin{array}{ll}1 & 1\end{array}$ & 11 & 11 & $\begin{array}{ll}1 & 1\end{array}$ & 1 & 11 & 1 & 1 & 21 & 21 & 11 & & $\begin{array}{ll}1 & 1\end{array}$ & $\begin{array}{ll}1 & 1\end{array}$ & 1 & 1 \\
\hline
\end{tabular}

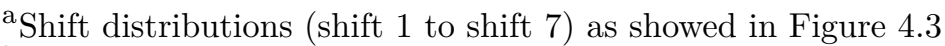

${ }^{\mathrm{b}}$ \#Physicians - Number of Physicians assigned to that shift

${ }^{\mathrm{c}}$ \#Sol - Number of different solutions

$\mathrm{d}$ \#SolD - Number of dominant solutions

${ }^{\mathrm{e} A N S}$ - Average Number of Solutions

Table F.12: Analysis of dominant solution from Monte Carlo Sampling method using 900 scenarios

\begin{tabular}{|c|c|c|c|c|c|c|c|c|c|c|c|c|c|c|c|c|c|c|c|c|c|c|c|c|c|c|c|c|c|c|c|c|c|c|c|}
\hline \multirow[b]{2}{*}{$\begin{array}{l}\text { Shifts/ } \\
\text { \#Physicians }\end{array}$} & \multicolumn{5}{|c|}{ Monday } & \multicolumn{5}{|c|}{ Tuesday } & \multicolumn{5}{|c|}{ Wednesday } & \multicolumn{5}{|c|}{ Thursday } & \multicolumn{5}{|c|}{ Friday } & \multicolumn{5}{|c|}{ Saturday } & \multicolumn{5}{|c|}{ Sunday } \\
\hline & 12 & 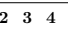 & & & 1 & 2 & 34 & 45 & & 7 & 12 & 3 & 45 & & $\begin{array}{ll}7 & 1\end{array}$ & 12 & 3 & 45 & 6 & 7 & 2 & 23 & 4 & 56 & 6 & 1 & 2 & 345 & 56 & $\begin{array}{lll}67 & 7\end{array}$ & 12 & 23 & 45 & 67 & \\
\hline 0 & & & & & U & 0 & 13 & 030 & 0 & 0 & $\begin{array}{ll}0 & 0\end{array}$ & 24 & 015 & 0 & 0 & $\begin{array}{ll}0 & 0 \\
\end{array}$ & 929 & 014 & & 0 & 0 & 011 & 104 & 42 & $\begin{array}{ll}1 & 0\end{array}$ & 00 & 01 & $\begin{array}{lll}18 & 02 \\
\end{array}$ & 251 & $\begin{array}{ll}4 & 0\end{array}$ & $\begin{array}{ll}0 & 0 \\
\end{array}$ & 022 & 032 & & \\
\hline 1 & & $\begin{array}{lll}0 & 18 & 0\end{array}$ & 433 & & & & 32 & & & & & 24 & 035 & & & & 20 & 036 & & & & 037 & & & & & & & 232 & & & & & & \\
\hline 2 & & & 01 & & & 35 & & & 37 & & & 1 & 00 & & 0 & 012 & & 00 & & & & & 10 & & & & & $\begin{array}{lll}1 & 0 \\
0 & 0\end{array}$ & 1 & & 030 & 00 & & & 0 \\
\hline 3 & 039 & $\begin{array}{lll}39 & 0 & 0\end{array}$ & 0 & & $\begin{array}{lll}0 & 0\end{array}$ & 15 & 0 & $\begin{array}{ll}0 & 0\end{array}$ & 0 & 0 & 040 & 0 & $\begin{array}{ll}0 & 0\end{array}$ & 0 & 0 & 037 & & $\begin{array}{lll}0 & 0\end{array}$ & 0 & 0 & 0 & & $\begin{array}{lll}0 & 0\end{array}$ & 0 & $\begin{array}{ll}0 & 0\end{array}$ & & 18 & $\begin{array}{lll}0 & 0\end{array}$ & 0 & & 020 & $\begin{array}{llllllll}0 & 0 & 0\end{array}$ & $\begin{array}{ll}0 & 0\end{array}$ & 0 & 0 \\
\hline 4 & $\begin{array}{ll}0 & 6 \\
0\end{array}$ & $\begin{array}{lll}6 & 0 & 0\end{array}$ & & & $\begin{array}{ll}0 & 0 \\
0\end{array}$ & 0 & 0 & & & 0 & $\begin{array}{ll}0 & 0 \\
\end{array}$ & 01 & 130 & & 0 & 21 & $\begin{array}{lll}1 & 0\end{array}$ & $\begin{array}{ll}10 & 0\end{array}$ & 0 & 0 & 1 & & 027 & & $0 \longdiv { 3 3 }$ & $3 \quad 9$ & 0 & 015 & & 0151 & 110 & $\begin{array}{lll}0 & 0\end{array}$ & 70 & & 10 \\
\hline 5 & $\begin{array}{ll}9 & 0 \\
\end{array}$ & $\begin{array}{llll}0 & 0 & 11\end{array}$ & & & 215 & & 02 & & & & & 03 & & & 504 & & & & & 50 & & & 021 & 0 & & 741 & 0 & 034 & & 0353 & $\begin{array}{ll}39 & 0\end{array}$ & & 310 & & 4 \\
\hline 6 & 410 & $\begin{array}{lll}0 & 0 & 35\end{array}$ & 0 & & 835 & 50 & 02 & & & & $\begin{array}{ll}6 & 0\end{array}$ & & & & 0 & 30 & & 40 & & 0 & 0 & $\begin{array}{ll}0 & 0 \\
\end{array}$ & 02 & 0 & & $\begin{array}{ll}0 & 0\end{array}$ & 0 & 01 & 0 & $\begin{array}{lll}0 & 0\end{array}$ & 00 & $\begin{array}{lll}0 & 0\end{array}$ & 120 & & 0 \\
\hline 7 & $\begin{array}{ll}0 & 0\end{array}$ & $\begin{array}{lll}0 & 0 & 4\end{array}$ & 0 & 0 & $\begin{array}{lll}0 & 0\end{array}$ & 0 & 0 & $\begin{array}{ll}0 & 0\end{array}$ & 0 & 0 & $\begin{array}{ll}0 & 0 \\
\end{array}$ & 0 & $\begin{array}{ll}0 & 0\end{array}$ & 0 & 0 & $\begin{array}{ll}0 & 0\end{array}$ & $\begin{array}{lll}0 & 0 \\
\end{array}$ & $\begin{array}{ll}0 & 0\end{array}$ & 0 & 0 & 0 & $\begin{array}{ll}0 & 0 \\
\end{array}$ & $\begin{array}{ll}0 & 0\end{array}$ & 0 & 0 & $\begin{array}{ll}0 & 0\end{array}$ & 0 & $\begin{array}{ll}0 & 0\end{array}$ & 0 & $\begin{array}{ll}0 & 0 \\
\end{array}$ & $\begin{array}{ll}0 & 0\end{array}$ & $\begin{array}{lll}0 & 0\end{array}$ & $\begin{array}{ll}0 & 0\end{array}$ & 0 & $0 \widehat{\mathrm{ANS}}$ \\
\hline $4 \mathrm{Sol}$ & $\begin{array}{ll}2 & 3 \\
1 & 1\end{array}$ & \begin{tabular}{|lll}
3 & 2 & 3 \\
1 & 1 & 1
\end{tabular} & 2 & & 2 & 22 & 3 & $\begin{array}{ll}3 & 3 \\
2 & \end{array}$ & 2 & 1 & 22 & 3 & $\begin{array}{ll}32 \\
1\end{array}$ & 2 & 1 & $\begin{array}{ll}33 \\
\end{array}$ & 32 & $\begin{array}{ll}3 & 2 \\
1\end{array}$ & 22 & 1 & 2 & 23 & $\begin{array}{ll}33 \\
1\end{array}$ & 2 & 3 & 22 & 2 & $\begin{array}{ll}3 & 3 \\
1\end{array}$ & 3 & $\begin{array}{ll}3 & 2 \\
1 & \end{array}$ & $\begin{array}{ll}22 \\
1\end{array}$ & 22 & $\begin{array}{ll}3 & 2 \\
1 & \end{array}$ & 23 & $\begin{array}{ll}2 & 2.3 \\
\end{array}$ \\
\hline
\end{tabular}

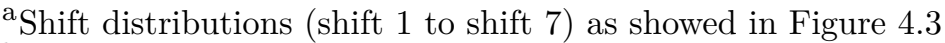

b \#Physicians - Number of Physicians assigned to that shift

${ }^{\mathrm{c}}$ \#Sol - Number of different solutions

$\mathrm{d}$ \#SolD - Number of dominant solutions

${ }^{\mathrm{e}} \mathrm{ANS}$ - Average Number of Solutions

Table F.13: Analysis of dominant solution from Monte Carlo Sampling method using 1000 scenarios

\begin{tabular}{|c|c|c|c|c|c|c|c|c|c|c|c|c|c|c|c|c|c|c|c|c|c|c|c|c|c|c|c|c|c|c|c|c|c|c|c|c|c|c|c|}
\hline \multirow[b]{2}{*}{$\begin{array}{l}\text { Shifts/ } \\
\text { \#Physicians }\end{array}$} & \multicolumn{6}{|c|}{ Monday } & \multicolumn{5}{|c|}{ Tuesday } & \multicolumn{6}{|c|}{ Wednesday } & \multicolumn{5}{|c|}{ Thursday } & \multicolumn{5}{|c|}{ Friday } & \multicolumn{5}{|c|}{ Saturday } & \multicolumn{6}{|c|}{ Sunday } & \\
\hline & 1 & 2 & 34 & & 6 & & 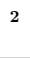 & 3 & 45 & & 7 & & 23 & 4 & & 67 & & 2 & & & & & & & & & & & & & & & & & & 5 & & & \\
\hline 0 & 0 & 03 & 5 & 5 & 0 & 0 & 0 & 12 & 02 & & 0 & 0 & 036 & 0 & 12 & $\begin{array}{ll}0 & 0 \\
0\end{array}$ & 0 & 02 & 260 & 10 & 0 & 0 & 0 & & & 45 & $\begin{array}{lll}0 & 0\end{array}$ & 0 & 023 & 30 & 23 & 190 & 0 & 02 & 210 & 35 & & & \\
\hline 1 & & 01 & 5 & 43 & 37 & 0 & 0 & 34 & 02 & 19 & 0 & 0 & 012 & 20 & 374 & 410 & 0 & 02 & 230 & 40 & 49 & 0 & 0 & 36 & 0 & 41 & 170 & 0 & 025 & 50 & 27 & 260 & 0 & 02 & 28 & 13 & 36 & & \\
\hline 2 & & 2 & 0 & 2 & & 0 & 27 & 4 & 0 & 141 & 0 & 0 & 72 & 20 & 0 & $\begin{array}{ll}9 & 0 \\
\end{array}$ & 0 & 6 & $\begin{array}{ll}0 & 0\end{array}$ & 0 & 1 & 0 & $04 \mathrm{t}$ & 64 & 40 & 03 & 330 & 02 & 21 & $\begin{array}{ll}1 & 0\end{array}$ & 0 & 40 & $0 \quad 0$ & 37 & 0 & 1 & 14 & & \\
\hline 3 & 0 & 41 & 0 & 0 & 0 & o & 23 & 0 & 0 & 0 & 0 & 04 & 430 & 0 & 0 & $\begin{array}{lll}0 & 0\end{array}$ & 0 & 43 & $\begin{array}{ll}0 & 0\end{array}$ & 0 & 0 & 0 & 0 & 40 & 0 & 0 & $\begin{array}{ll}0 & 0\end{array}$ & 02 & 28 & $\begin{array}{ll}0 & 0\end{array}$ & 0 & $\begin{array}{lll}0 & 0\end{array}$ & $\begin{array}{lll}0 & 0\end{array}$ & 11 & 0 & 0 & & 0 & \\
\hline 4 & 0 & 7 & 0 & 0 & 0 & 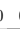 & 0 & 0 & 2 & ) & 0 & 0 & $\begin{array}{ll}0 & 0\end{array}$ & 8 & 0 & $\begin{array}{ll}0 & 0\end{array}$ & 4 & 1 & 017 & 0 & 0 & 0 & 21 & 00 & 29 & 0 & 038 & 10 & 1 & $\begin{array}{ll}0 & 8\end{array}$ & 0 & 010 & 09 & 2 & 04 & 10 & 01 & & \\
\hline 5 & & 0 & 01 & & 0 & 32 & & 0 & 4 & & 50 & & $\begin{array}{ll}0 & 0\end{array}$ & 037 & 0 & 050 & 46 & 0 & 029 & 0 & & 504 & 181 & & 20 & 0 & $\begin{array}{lll}0 & 12\end{array}$ & 40 & 0 & 041 & 0 & 040 & 41 & 0 & $0 \longdiv { 3 5 }$ & 0 & 03 & 35 & \\
\hline 6 & 41 & 0 & 03 & & & 72 & 0 & 0 & & 0 & 0 & 4 & $0 \quad 0$ & 5 & 0 & $\begin{array}{ll}0 & 0\end{array}$ & 0 & 0 & $\begin{array}{ll}04 \\
4\end{array}$ & 0 & 0 & 0 & 0 & & 1 & 0 & $\begin{array}{ll}0 & 0\end{array}$ & 0 & 0 & $\begin{array}{ll}0 & 1\end{array}$ & 0 & 0 & 0 & 0 & 011 & 10 & & 0 & \\
\hline 7 & 0 & & & 0 & 0 & 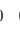 & 0 & 0 & 0 & 0 & 0 & 0 & 0 & 0 & 0 & 00 & 0 & 0 & & 0 & 0 & 0 & 0 & & & 0 & $\begin{array}{ll}0 & 0\end{array}$ & 0 & 0 & $\begin{array}{ll}0 & 0\end{array}$ & 0 & 0 & 00 & 0 & 0 & 0 & 0 & & $\overline{\mathrm{ANS}}$ \\
\hline \#Sol & 2 & 3 & 2 & 3 & 2 & 2 & 2 & 3 & 3 & 32 & 1 & 2 & 23 & 33 & 2 & 21 & 2 & 3 & 2 & 32 & 2 & 1 & 2 & 2 & 3 & 2 & 22 & 2 & 3 & $\begin{array}{ll}33 \\
\end{array}$ & 2 & 32 & 22 & 3 & 2 & 3 & 2 & 2 & 2.3 \\
\hline \#SolD & 1 & 1 & & 1 & 1 & 1 & 1 & 1 & 1 & 1 & 1 & 1 & 11 & 11 & 1 & 11 & 1 & 1 & 1 & 1 & 1 & 1 & 1 & 11 & 11 & 1 & 11 & 1 & 1 & 21 & & 1 & 1 & 1 & 1 & 11 & & & 1.0 \\
\hline
\end{tabular}

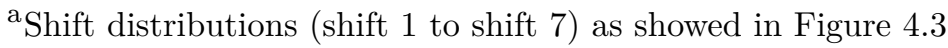

$\mathrm{b}$ \#Physicians - Number of Physicians assigned to that shift

${ }^{\mathrm{c}}$ \#Sol - Number of different solutions

d \#SolD - Number of dominant solutions

${ }^{\mathrm{e}}$ ANS - Average Number of Solutions 
Table F.14: Analysis of dominant solution from Latin Hypercube Sampling method using 100 scenarios

\begin{tabular}{|c|c|c|c|c|c|c|c|c|c|c|c|c|c|c|c|c|c|c|c|c|c|c|c|c|c|c|c|c|c|c|c|c|c|c|c|c|c|c|c|}
\hline \multirow{2}{*}{$\begin{array}{l}\text { Shifts } / \\
\text { \#Physicians }^{\mathrm{b}}\end{array}$} & \multicolumn{5}{|c|}{ Monday } & \multicolumn{6}{|c|}{ Tuesday } & \multicolumn{5}{|c|}{ Wednesday } & \multicolumn{6}{|c|}{ Thursday } & \multicolumn{5}{|c|}{ Friday } & \multicolumn{5}{|c|}{ Saturday } & \multicolumn{6}{|c|}{ Sunday } & \\
\hline & 1 & $\begin{array}{ll}2 & 3 \\
\end{array}$ & \multicolumn{2}{|c|}{$\begin{array}{lll}4 & 5 & 6\end{array}$} & \multicolumn{2}{|c|}{67} & & 123 & \multicolumn{2}{|c|}{345} & & 1 & \multicolumn{3}{|c|}{$\begin{array}{llll}2 & 3 & 4 & 5\end{array}$} & & & & \multicolumn{3}{|c|}{$\begin{array}{lll}3 & 4 & 5\end{array}$} & & & & 45 & & & & & & & & & & & 45 & & & \\
\hline 0 & 0 & 01 & 140 & 13 & 0 & 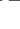 & 00 & 16 & 022 & 24 & & 0 & $\begin{array}{ll}0 & 9 \\
\end{array}$ & 902 & 21 & 10 & & $\begin{array}{ll}0 & 0\end{array}$ & 10 & 024 & 0 & & 0 & 021 & 03 & $35 \quad 5$ & 0 & & 028 & 80 & 151 & 190 & & $\begin{array}{lll}0 & 0\end{array}$ & 28 & 012 & 24 & & \\
\hline 1 & 0 & 12 & 290 & $24 ?$ & 30 & & $\begin{array}{ll}0 & 1\end{array}$ & 27 & 017 & 717 & & 0 & 331 & 102 & 233 & 60 & & $\begin{array}{ll}0 & 1\end{array}$ & 36 & 022 & 42 & & 0 & 225 & 01 & 1319 & 0 & & 220 & 0 & 282 & 250 & & $\begin{array}{lll}0 & 3\end{array}$ & 18 & 030 & 030 & 0 & \\
\hline 2 & 0 & 9 & 70 & 132 & & & 015 & 7 & 010 & 029 & & 02 & 239 & 90 & 61 : & & & 021 & 3 & $\begin{array}{lll}0 & 4\end{array}$ & 8 & & 03 & $34 \quad 4$ & 0 & 224 & 0 & & 192 & 20 & 7 & $\begin{array}{ll}6 & 0\end{array}$ & & 017 & & $\begin{array}{ll}0 & 8 \\
0\end{array}$ & 816 & 0 & \\
\hline 3 & 03 & & $\begin{array}{ll}0 & 1\end{array}$ & 0 & 0 & & 032 & 0 & 0 & 10 & & & & 15 & 0 & 10 & & 025 & 1 & 20 & 0 & 0 & 01 & & 2 & $\begin{array}{ll}0 & 2\end{array}$ & 0 & & 26 c & $\begin{array}{lll}0 & 3\end{array}$ & 0 & $\begin{array}{ll}0 & 0\end{array}$ & & & 0 & 20 & $\begin{array}{lll}0 & 0\end{array}$ & 0 & \\
\hline 4 & 0 & & $\begin{array}{ll}0 & 7\end{array}$ & 0 & 0 & & 02 & & 13 & 0 & 0 & 0 & $\begin{array}{ll}2 & 0\end{array}$ & 23 & 0 & $\begin{array}{ll}0 & 0\end{array}$ & & 83 & 02 & 230 & 0 & & 6 & $\begin{array}{ll}0 & 0\end{array}$ & 28 & $\begin{array}{lll}0 & 0\end{array}$ & 34 & 17 & 30 & 020 & 0 & $0 \longdiv { 2 2 }$ & & 05 & & 200 & $\begin{array}{lll}0 & 0\end{array}$ & 19 & \\
\hline 5 & 24 & 0 & 020 & 0 & 01 & 3 & 50 & 0 & $26 \mathrm{c}$ & $\begin{array}{ll}0 & 0 \\
\end{array}$ & 42 & 33 & $\begin{array}{ll}0 & 0\end{array}$ & 15 & 0 & $0 \longdiv { 4 7 }$ & 37 & 370 & 02 & 230 & 04 & 194 & 40 & $\begin{array}{ll}0 & 0\end{array}$ & 18 & $\begin{array}{ll}0 & 0\end{array}$ & 16 & 31 & 00 & 023 & 0 & 028 & 26 & 60 & & 220 & $\begin{array}{lll}0 & 0\end{array}$ & 31 & \\
\hline 6 & 21 & 0 & 017 & 0 & 03 & & 50 & & 10 & $\begin{array}{ll}0 & 0\end{array}$ & & 16 & $\begin{array}{ll}0 & 0\end{array}$ & 97 & 0 & 03 & & $\begin{array}{ll}40 \\
4\end{array}$ & 0 & 20 & 0 & 1 & 4 & $\begin{array}{ll}0 & 0\end{array}$ & 2 & $\begin{array}{ll}0 & 0\end{array}$ & 0 & 2 & 0 & $\begin{array}{ll}0 \quad 4 \\
0\end{array}$ & 0 & $\begin{array}{ll}0 & 0\end{array}$ & & $\begin{array}{ll}3 & 0\end{array}$ & & 60 & $\begin{array}{lll}0 & 0\end{array}$ & 0 & \\
\hline 7 & 5 & 0 & $\begin{array}{ll}0 & 5 \\
\end{array}$ & 0 & 0 & & $\begin{array}{ll}0 \quad 0 \\
\end{array}$ & 0 & 10 & $\begin{array}{ll}0 & 0 \\
\end{array}$ & 1 & 1 & $\begin{array}{ll}0 & 0 \\
\end{array}$ & $\begin{array}{ll}0 & 0 \\
\end{array}$ & 0 & 00 & & 10 & 0 & $\begin{array}{ll}0 & 0 \\
\end{array}$ & 0 & 0 & 0 & $\begin{array}{ll}0 & 0 \\
\end{array}$ & 0 & $\begin{array}{ll}0 & 0 \\
\end{array}$ & 0 & 0 & 0 & $\begin{array}{ll}0 & 0 \\
\end{array}$ & 0 & $\begin{array}{ll}0 & 0 \\
\end{array}$ & & 10 & & 00 & $\begin{array}{ll}0 & 0 \\
\end{array}$ & & $\mathrm{ANS}^{\mathrm{e}}$ \\
\hline \#: & 3 & 4 & $\begin{array}{ll}3 & 5\end{array}$ & 3 & 2 & & 24 & 3 & 4 & 43 & 3 & 3 & 44 & 44 & 3 & 42 & & 44 & & 43 & 2 & 2 & 3 & & 4 & $\begin{array}{ll}34 \\
\end{array}$ & 12 & 3 & 43 & $\begin{array}{ll}34 \\
\end{array}$ & 3 & 32 & 24 & 44 & & 43 & $\begin{array}{ll}3 & 3\end{array}$ & 2 & 3.2 \\
\hline \#SolD & 2 & 1 & $\begin{array}{ll}12 \\
\end{array}$ & 3 & 1 & & 11 & 1 & 12 & 21 & 1 & 1 & $\begin{array}{ll}2 & 1\end{array}$ & 12 & 2 & 11 & & $\begin{array}{ll}12 \\
\end{array}$ & 1 & 22 & 1 & 1 & 1 & $\begin{array}{ll}12 \\
\end{array}$ & 1 & 12 & 1 & 1 & 1 & 12 & 1 & 21 & 11 & 12 & & 21 & 11 & 1 & 1.3 \\
\hline
\end{tabular}

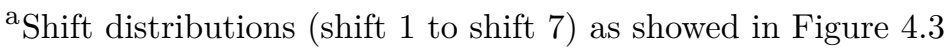

$\mathrm{b}$ \#Physicians - Number of Physicians assigned to that shift

${ }^{\mathrm{c}}$ \#Sol - Number of different solutions

d\#SolD - Number of dominant solutions

${ }^{\mathrm{e}}$ ANS - Average Number of Solutions

Table F.15: Analysis of dominant solution from Latin Hypercube Sampling method using 200 scenarios

\begin{tabular}{|c|c|c|c|c|c|c|c|c|c|c|c|c|c|c|c|c|c|c|c|c|c|c|c|c|c|c|c|c|c|c|c|c|c|c|c|c|c|}
\hline \multirow[b]{2}{*}{$\begin{array}{l}\text { Shifts/ } \\
\text { \#Physicians }\end{array}$} & \multicolumn{5}{|c|}{ Monday } & \multicolumn{5}{|c|}{ Tuesday } & \multicolumn{5}{|c|}{ Wednesday } & \multicolumn{6}{|c|}{ Thursday } & \multicolumn{6}{|c|}{ Friday } & \multicolumn{5}{|c|}{ Saturday } & \multicolumn{4}{|c|}{ Sunday } & \\
\hline & 12 & $\begin{array}{ll}23 \\
\end{array}$ & 4 & $\begin{array}{ll}56 \\
\end{array}$ & & 1 & 23 & 34 & & 67 & 1 & 23 & 34 & 5 & & & 12 & 3 & & & 7 & 1 & & & & & & & 23 & & 6 & & $\begin{array}{ll}12 \\
\end{array}$ & & 45 & & 7 \\
\hline 0 & & & & $10 \quad 0$ & & 0 & $\begin{array}{lll}01 \\
0\end{array}$ & $\begin{array}{lll}20 & 0\end{array}$ & $20 \quad 0$ & & 0 & 01 & & 20 & $0 \quad 0$ & & $\begin{array}{ll}0 & 0 \\
\end{array}$ & 16 & 019 & 90 & & 0 & 02 & 2105 & 36 & & & & $\begin{array}{ll}023 \\
\end{array}$ & 018 & & & $\begin{array}{ll}0 & 0 \\
0\end{array}$ & 023 & & 27 & \\
\hline 1 & 0 & 024 & & 3522 & & & 13 & & 3013 & & 0 & 02 & & 303 & & & & 34 & 031 & 149 & & & & & 141 & & & & 025 & 029 & & & & 025 & & 2336 & \\
\hline 2 & 0 & & & 527 & & & 24 & $\begin{array}{lll}6 & 0\end{array}$ & 037 & & & & & 01 & & & 013 & & 00 & $\begin{array}{l}0.30 \\
0\end{array}$ & 0 & 0 & 36 & 20 & & 34 & & 025 & $25 \quad 2$ & 03 & & 0 & 033 & 32 & & 014 & 0 \\
\hline 3 & 03 & & 0 & $\begin{array}{lll}0 & 1\end{array}$ & & & 25 & $\begin{array}{lll}0 & 0\end{array}$ & 00 & & & 40 & & & & & 037 & & & & & & 12 & & & & & 022 & & & & & 014 & & & & 0 \\
\hline 4 & 01 & & & & 0 & 0 & & & & $\begin{array}{ll}0 & 0 \\
0\end{array}$ & & 2 & 021 & & & & 10 & 02 & 200 & 0 & & & 0 & 021 & 0 & 040 & & 143 & $\begin{array}{lll}3 & 0 & 1\end{array}$ & & & & $\begin{array}{ll}9 & 3\end{array}$ & & 100 & 0 & 13 \\
\hline 5 & 16 & $\begin{array}{ll}0 & 0\end{array}$ & 22 & & 27 & 22 & 0 & 034 & & 050 & 43 & 0 & 024 & & 050 & & & 02 & & & & & & 021 & 0 & 010 & & & $\begin{array}{lll}0 & 03\end{array}$ & 320 & & 313 & & $\begin{array}{lll}0 & 0\end{array}$ & 310 & $\begin{array}{ll}0 & 0 \\
\end{array}$ & 37 \\
\hline 6 & 30 & $\begin{array}{lll}0 & 0\end{array}$ & 22 & 0 & 2 & & & 0 & 0 & 0 & & 0 & 0 & 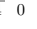 & 0 & & 50 & 0 & 5 & & 0 & 2 & 0 & $\begin{array}{ll}04 \\
4\end{array}$ & 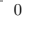 & $\begin{array}{ll}0 & 0 \\
\end{array}$ & & & & 0 & & & 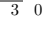 & $\begin{array}{lll}0 & 0 \\
0\end{array}$ & 0 & 0 & \\
\hline 7 & 4 & $\begin{array}{ll}0 & 0 \\
\end{array}$ & 5 & $\begin{array}{ll}0 & 0 \\
0\end{array}$ & 0 & 1 & 0 & $\begin{array}{lll}0 & 0 \\
\end{array}$ & 0 & $\begin{array}{ll}0 & 0 \\
\end{array}$ & 0 & 0 & $\begin{array}{lll}0 & 0 \\
\end{array}$ & 0 & 0 & 0 & $\begin{array}{ll}0 & 0 \\
0\end{array}$ & 0 & 0 & $\begin{array}{ll}0 & 0 \\
\end{array}$ & 0 & 0 & 0 & $\begin{array}{ll}0 & 0 \\
\end{array}$ & 0 & $\begin{array}{ll}0 & 0 \\
0\end{array}$ & & $\begin{array}{ll}0 & 0 \\
0\end{array}$ & $\begin{array}{ll}0 & 0 \\
0\end{array}$ & $\begin{array}{ll}0 & 0 \\
0\end{array}$ & & 0 & $\begin{array}{ll}0 & 0 \\
\end{array}$ & $\begin{array}{ll}0 & 0 \\
0\end{array}$ & $\begin{array}{lll}0 & 0 \\
0\end{array}$ & $\begin{array}{ll}0 & 0 \\
\end{array}$ & $0 \overline{\mathrm{AN}}$ \\
\hline & 3 & $\begin{array}{ll}33 \\
3\end{array}$ & 4 & 3 & 2 & 3 & 3 & $\begin{array}{ll}3 & 3\end{array}$ & 22 & 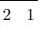 & 2 & 3 & $\begin{array}{ll}3 & 4\end{array}$ & 2 & 3 & & 32 & 2 & 3 & 2 & 1 & & 3 & $\begin{array}{|ll|}3 & 4\end{array}$ & 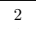 & 3 & & $\begin{array}{ll}23 \\
\end{array}$ & $\begin{array}{ll}3 & 3\end{array}$ & $\begin{array}{ll}3 & 3\end{array}$ & & 2 & $\begin{array}{lll}3 & 3\end{array}$ & $\begin{array}{ll}33 \\
\end{array}$ & 32 & 22 & 22 \\
\hline & 1 & 12 & 2 & 1 & & 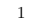 & 2 & 11 & 11 & $\begin{array}{ll}1 & 1\end{array}$ & 1 & 1 & 22 & 1 & 1 & & 1 & 1 & 21 & & 1 & 1 & . & 12 & 1 & 11 & 1 & 12 & 22 & $\begin{array}{lll}11 & 1\end{array}$ & 2 & 1 & 11 & 12 & 1 & $\begin{array}{lll}11 & 1\end{array}$ & 1 \\
\hline
\end{tabular}

Table F.16: Analysis of dominant solution from Latin Hypercube Sampling method using 300 scenarios

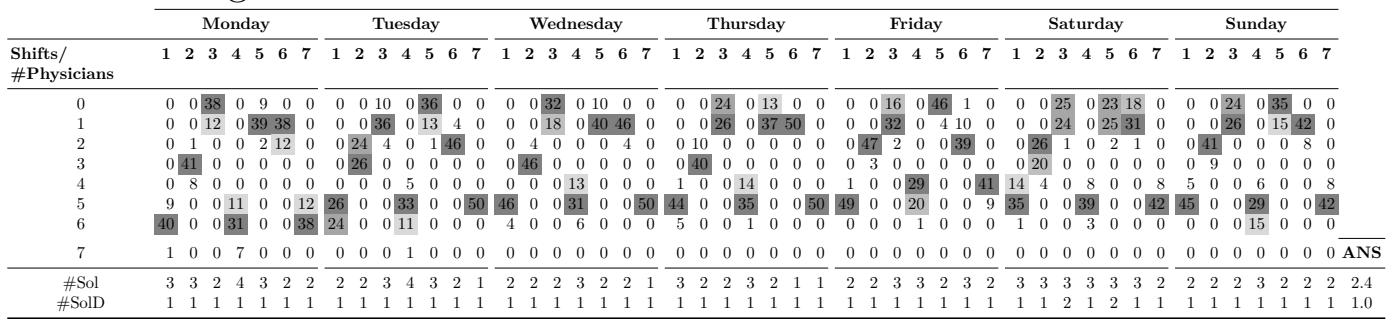


Table F.17: Analysis of dominant solution from Latin Hypercube Sampling method using 400 scenarios

\begin{tabular}{|c|c|c|c|c|c|c|c|c|c|c|c|c|c|c|c|c|c|c|c|c|c|c|c|c|c|c|c|c|c|c|c|c|c|c|c|c|c|c|}
\hline \multirow[b]{2}{*}{$\begin{array}{l}\text { Shifts/ } \\
\text { \#Physicians }\end{array}$} & \multicolumn{6}{|c|}{ Monday } & \multicolumn{5}{|c|}{ Tuesday } & \multicolumn{5}{|c|}{ Wednesday } & \multicolumn{6}{|c|}{ Thursday } & \multicolumn{6}{|c|}{ Friday } & \multicolumn{5}{|c|}{ Saturday } & \multicolumn{4}{|c|}{ Sunday } & \\
\hline & 1 & & $\begin{array}{ll}34 \\
\end{array}$ & & 67 & & 12 & 23 & 45 & & & & 2 & $\begin{array}{ll}34 \\
\end{array}$ & & & & & 23 & & & & & & & & & & & & & & & & $\begin{array}{ll}2 & 3 \\
\end{array}$ & 456 & & \\
\hline 0 & & 03 & $\begin{array}{ll}36 & 0 \\
\end{array}$ & 2 & 0 & & 0 & 013 & 03 & 370 & & 0 & & 340 & 1 & 0 & & 00 & 034 & 0 & 5 & 0 & & & 9 & 045 & & & & 016 & $\begin{array}{lll}6 & 0 & 31 \\
\end{array}$ & 3115 & & & $0 \longdiv { 2 2 }$ & 2034 & & \\
\hline 1 & & 01 & & 45 & 33 & & & 036 & & & $\begin{array}{ll}40 \\
0\end{array}$ & & & 160 & 494 & & & & 016 & & 455 & & & & 39 & & & & & 033 & & 1832 & & & 028 & 80164 & & \\
\hline 2 & & & 10 & 3 & 17 & & 022 & & 0 & 046 & & & 0 & $\begin{array}{lll}0 & 0\end{array}$ & 0 & & & & 20 & 0 & 0 & & & 049 & 2 & 00 & 45 & & & 311 & $\begin{array}{lll}1 & 0 & 1\end{array}$ & 13 & 0 & & $35 \quad 0$ & $\begin{array}{llll}0 & 0 & 0\end{array}$ & & 0 \\
\hline 3 & & 43 & $\begin{array}{ll}0 & 0\end{array}$ & 0 & & & $02 \xi$ & 280 & 0 & & & & 50 & $\begin{array}{lll}0 & 0\end{array}$ & & 0 & & 048 & 80 & 0 & & & & $\begin{array}{lll}0 & 1\end{array}$ & & 00 & & & 01 & 180 & & & & & $15 \quad 0$ & & & 0 \\
\hline 4 & & & $\begin{array}{ll}0 & 1\end{array}$ & & & & 0 & & & & & & 0 & 015 & & & & 00 & & 14 & & & & & & 340 & & & & & $\begin{array}{llll}0 & 5 & 0\end{array}$ & & & & & & & \\
\hline 5 & & 0 & 016 & & & & & & 35 & & 050 & & & 035 & & & & & & 33 & & & & & & & & & & $0 \quad 0$ & 045 & & & & & & $0 \longdiv { 4 8 }$ & 18 \\
\hline 6 & 42 & 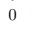 & 031 & & 03 & & 22 & $\begin{array}{ll}0 & 0\end{array}$ & 13 & 0 & 00 & & 0 & $\begin{array}{ll}0 & 0 \\
\end{array}$ & 0 & & & & $\begin{array}{ll}0 & 0 \\
\end{array}$ & 3 & 0 & 00 & & & & & 0 & & & & $\begin{array}{lll}0 & 0 & 0\end{array}$ & $\begin{array}{lll}0 & 0 \\
0\end{array}$ & & & $\begin{array}{ll}0 & 0\end{array}$ & $\begin{array}{lll}013 & 0\end{array}$ & 00 & \\
\hline 7 & 0 & 0 & $\begin{array}{ll}02 \\
02\end{array}$ & 20 & 0 & 0 & $0 \mathrm{c}$ & $\begin{array}{ll}0 & 0\end{array}$ & 0 & 00 & $\begin{array}{ll}0 & 0 \\
\end{array}$ & & $\begin{array}{ll}0 & 0\end{array}$ & $\begin{array}{ll}0 & 0\end{array}$ & 0 & 0 & 0 & 00 & $\begin{array}{ll}0 & 0\end{array}$ & 0 & 0 & 0 & & $\begin{array}{ll}0 & 0 \\
\end{array}$ & $\begin{array}{ll}0 & 0\end{array}$ & 00 & $\begin{array}{lll}0 & 0\end{array}$ & 0 & 0 & 00 & $\begin{array}{llll}0 & 0 & 0\end{array}$ & $\begin{array}{ll}0 & 0\end{array}$ & 00 & 0 & $\begin{array}{ll}0 & 0\end{array}$ & $\begin{array}{lll}0 & 0 & 0\end{array}$ & $\begin{array}{ll}0 & 0\end{array}$ & $0 \overline{\mathrm{ANS}}$ \\
\hline$\# \mathrm{~S} 0 \mathrm{l}$ & 2 & 2 & $\begin{array}{ll}34 \\
\end{array}$ & 43 & 2 & & 2 & 23 & 3 & 22 & 21 & & 11 & 22 & 22 & 2 & $\overline{1}$ & 12 & 22 & 3 & 2 & 1 & & 2 & 23 & 32 & 22 & 2 & 2 & 33 & 32 & $\begin{array}{ll}33 \\
3\end{array}$ & 32 & 2 & 22 & 232 & 2 & $\begin{array}{ll}2 & 2.1 \\
\end{array}$ \\
\hline & & & 1 & 1 & & & 1 & & 1 & 11 & & & 1 & $\begin{array}{ll}1 & 1\end{array}$ & 1 & & & & 1 & & 1 & & & & 1 & $\begin{array}{lll}11 & 1\end{array}$ & & & & & $\begin{array}{lll}1 & 1\end{array}$ & $\begin{array}{ll}1 & 1\end{array}$ & & 1 & 1 & $\begin{array}{lll}1 & 1 & 1\end{array}$ & & \\
\hline
\end{tabular}

Table F.18: Analysis of dominant solution from Latin Hypercube Sampling method using 500 scenarios

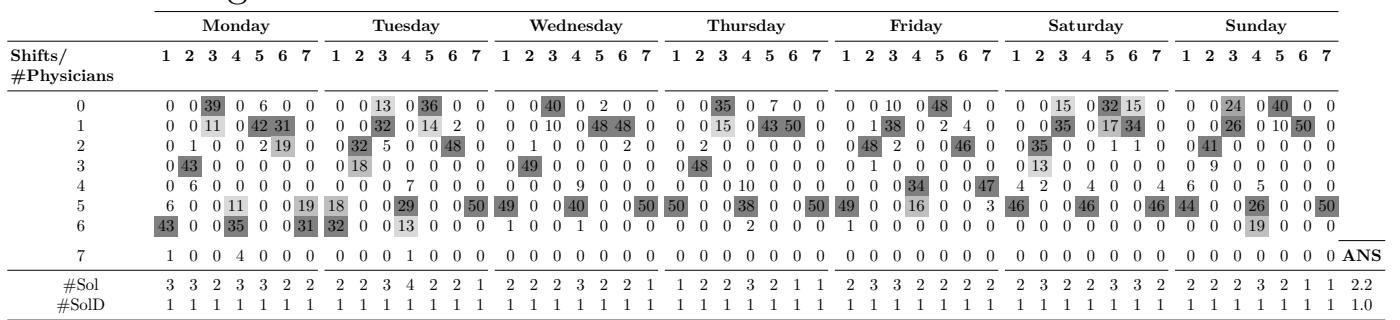

Table F.19: Analysis of dominant solution from Latin Hypercube Sampling method using 600 scenarios

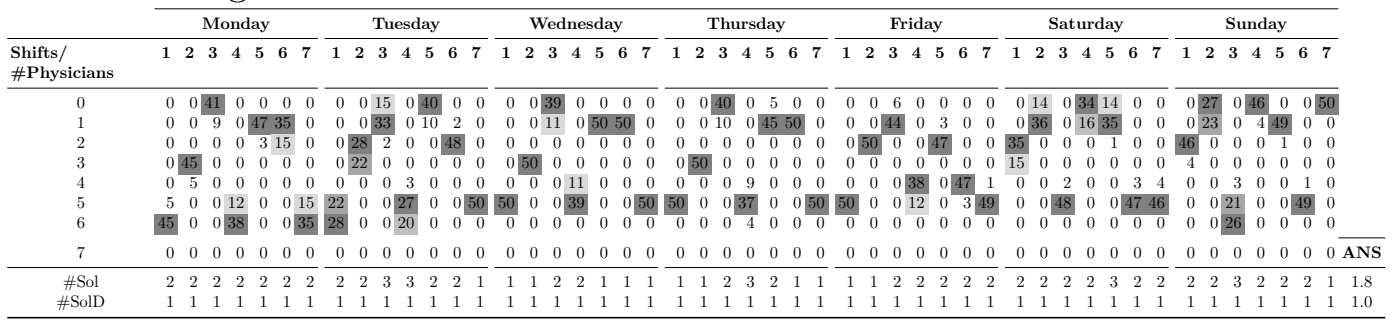

Table F.20: Analysis of dominant solution from Latin Hypercube Sampling method using 700 scenarios

\begin{tabular}{|c|c|c|c|c|c|c|c|c|c|c|c|c|c|c|c|c|c|c|c|c|c|c|c|c|c|c|c|c|c|c|c|c|c|c|c|c|c|c|c|}
\hline \multirow[b]{2}{*}{$\begin{array}{l}\text { Shifts/ } \\
\text { \#Physicians }\end{array}$} & \multicolumn{5}{|c|}{ Monday } & \multicolumn{5}{|c|}{ Tuesday } & & \multicolumn{5}{|c|}{ Wednesday } & \multicolumn{6}{|c|}{ Thursday } & \multicolumn{6}{|c|}{ Friday } & \multicolumn{6}{|c|}{ Saturday } & \multicolumn{4}{|c|}{ Sunday } & \\
\hline & 12 & 23 & 4 & 56 & & 1 & 23 & 4 & & 67 & & 12 & 3 & 45 & & & 1 & 2 & 34 & 4 & & & 1 & 2 & 3 & 45 & & 7 & 1 & 2 & $\begin{array}{ll}34 \\
\end{array}$ & 5 & & & 12 & 23 & 45 & 6 & \\
\hline 0 & 04 & & 3 & & & 0 & 11 & & & 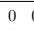 & & 045 & & 00 & $\begin{array}{ll}0 & 0 \\
\end{array}$ & 0 & & 38 & 0 & 4 & 00 & $\overline{0}$ & & 6 & 05 & 50 & $\begin{array}{ll}0 & 0\end{array}$ & 0 & & 7 & 042 & 7 & 0 & & 021 & 104 & $\begin{array}{ll}45 & 0 \\
\end{array}$ & & \\
\hline 1 & 0 & & 474 & & & & 38 & & & & & 05 & & 5050 & & & & 12 & 04 & 65 & & & & 44 & & & & & & 43 & & & & & 029 & & & & \\
\hline 2 & & & 0 & 70 & & & 1 & & & & & & & 00 & $\begin{array}{ll}0 & 0\end{array}$ & & & 20 & 0 & 0 & $\begin{array}{ll}0 & 0\end{array}$ & & & & & 050 & & & & & $\begin{array}{ll}0 & 0\end{array}$ & 0 & & & & & & & \\
\hline $\begin{array}{l}3 \\
4\end{array}$ & $\frac{45}{5}$ & & 0 & 0 & 0 & 16 & 0 & $\begin{array}{lll}0 & 0 \\
3 & 0\end{array}$ & & 0 & & & $\begin{array}{ll}0 & 0 \\
0 & 5\end{array}$ & $\begin{array}{lll}0 & 0 \\
0 & 0 & 0\end{array}$ & $\begin{array}{ll}0 & 0 \\
0 & 0\end{array}$ & & 48 & & & 0 & & 0 & & & 0 & & & 0 & & & $\begin{array}{ll}0 & 0 \\
1 & 0\end{array}$ & & 0 & 0 & & $\begin{array}{ll}0 & 0 \\
0 & 0\end{array}$ & & & 0 \\
\hline $\begin{array}{l}4 \\
5\end{array}$ & 0 & $\begin{array}{ll}0 \\
0 & 5\end{array}$ & & & 8 & & 03 & & & 505 & & $\begin{array}{lll}0 & 0 \\
0\end{array}$ & 0 & & 050 & 50 & & & 100 & & $\begin{array}{l}010 \\
050\end{array}$ & 50 & & & 10 & $\begin{array}{l}0 \\
0\end{array}$ & & 47 & & & $\begin{array}{ll}1 & 0 \\
49 & 0\end{array}$ & & 50 & $\begin{array}{l}3 \\
47 \\
47\end{array}$ & & $\begin{array}{ll}0 & 2 \\
0 & 30\end{array}$ & & 50 & 0 \\
\hline 6 & 0 & 042 & & & 34 & 0 & 01 & & & 0 & & 00 & 0 & 0 & 00 & 0 & 0 & & 2 & 0 & & 0 & 0 & & 0 & 0 & $\begin{array}{lll}0 & 0\end{array}$ & 0 & 0 & & $\begin{array}{lll}0 & 0\end{array}$ & 0 & 0 & 0 & 0 & 018 & $\begin{array}{lll}0 & 0\end{array}$ & 0 & 0 \\
\hline 7 & 0 & $\begin{array}{ll}0 & 3 \\
\end{array}$ & 0 & $0 \mathrm{c}$ & 0 & 0 & 0 & $\begin{array}{ll}0 & 0\end{array}$ & 0 & 0 & 0 & $\begin{array}{ll}0 & 0\end{array}$ & $\begin{array}{ll}0 & 0 \\
\end{array}$ & 0 & $\begin{array}{lll}0 & 0\end{array}$ & 0 & 0 & 0 & 0 & 0 & $\begin{array}{ll}0 & 0 \\
0\end{array}$ & 0 & 0 & 0 & 0 & 0 & $\begin{array}{ll}0 & 0\end{array}$ & 0 & 0 & 0 & $\begin{array}{ll}0 & 0\end{array}$ & 0 & 0 & 0 & $\begin{array}{ll}0 & 0 \\
0\end{array}$ & $\begin{array}{ll}0 & 0\end{array}$ & $\begin{array}{ll}0 & 0\end{array}$ & 0 & $0 \overline{\mathrm{ANS}}$ \\
\hline & 2 & 23 & 2 & 22 & & 2 & & $\begin{array}{ll}32 \\
2\end{array}$ & 2 & 1 & & & 22 & 1 & & 1 & & & & 2 & & 1 & & & & 1 & & & & & $\begin{array}{ll}22 \\
2\end{array}$ & & & & & & 21 & & \\
\hline & 1 & & 1 & & & 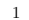 & 1 & 1 & 1 & 1 & & 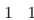 & 11 & 1 & 11 & 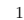 & 1 & 1 & 1 & 1 & 1 & 1 & 1 & 1 & 1 & 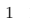 & 11 & 1 & 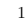 & 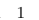 & $\begin{array}{lll}11 \\
\end{array}$ & 1 & 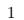 & 1 & 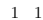 & 11 & 11 & 11 & 1 \\
\hline
\end{tabular}

Table F.21: Analysis of dominant solution from Latin Hypercube Sampling method using 800 scenarios

\begin{tabular}{|c|c|c|c|c|c|c|c|c|c|c|c|c|c|c|c|c|c|c|c|c|c|c|c|c|c|c|c|c|c|c|c|c|c|c|c|c|c|}
\hline \multirow[b]{2}{*}{$\begin{array}{l}\text { Shifts/ } \\
\text { \#Physicians }\end{array}$} & \multicolumn{5}{|c|}{ Monday } & \multicolumn{5}{|c|}{ Tuesday } & & \multicolumn{5}{|c|}{ Wednesday } & \multicolumn{5}{|c|}{ Thursday } & & \multicolumn{5}{|c|}{ Friday } & \multicolumn{5}{|c|}{ Saturday } & \multicolumn{4}{|c|}{ Sunday } & \\
\hline & 1 & 23 & 4 & 56 & & 1 & 2 & $\begin{array}{ll}34 \\
\end{array}$ & 5 & 67 & & 2 & 3 & 45 & & 67 & & 2 & 34 & & 67 & & & & 45 & & & & 23 & 45 & & 7 & & $\begin{array}{ll}2 & 3 \\
\end{array}$ & 45 & 56 & \\
\hline 0 & 0 & 045 & & $\begin{array}{ll}0 & 0 \\
0\end{array}$ & & & 0 & 70 & 49 & 0 & & 00 & 050 & 0 & $\begin{array}{lll}0 & 0 \\
\end{array}$ & & 0 & & 500 & 0 & 0 & & & $\begin{array}{lll}0 & 0\end{array}$ & 050 & 50 & & & $\begin{array}{ll}0 & 6 \\
\end{array}$ & \begin{tabular}{|ll|l}
6 & 0 & 44
\end{tabular} & 46 & & & 025 & 5050 & 50 & \\
\hline 1 & & & & 5044 & & & & & & & & & $\begin{array}{ll}0 & 0\end{array}$ & & 5050 & & & & & 505 & & & & 050 & 00 & & 0 & & 044 & $\begin{array}{lll}4 & 0 & 6\end{array}$ & & 0 & & 025 & & & \\
\hline 2 & & & 0 & $\begin{array}{lll}0 & 6 \\
\end{array}$ & & & 31 & 0 & & 49 & & & $\begin{array}{lll}0 & 0\end{array}$ & & $\begin{array}{ll}0 & 0 \\
\end{array}$ & & & & & 0 & & & & $\begin{array}{lll}0 & 0\end{array}$ & 0 & & 0 & & $44 \quad 0$ & $\begin{array}{lll}0 & 0 & 0\end{array}$ & 0 & 0 & & $\begin{array}{ll}490 \\
0\end{array}$ & $\begin{array}{lll}0 & 0 \\
0 & 0\end{array}$ & 00 & 0 \\
\hline 3 & 05 & & 0 & & & & 19 & $\begin{array}{lll}0 & 0\end{array}$ & & 0 & & 056 & 50 & & & $\begin{array}{lll}0 & 0 \\
0\end{array}$ & & 50 & & & 0 & & & & 0 & & 0 & & & 0 & & 0 & & & & & 0 \\
\hline 4 & 0 & & 0 & 00 & & & 0 & $\begin{array}{ll}0 & 0 \\
\end{array}$ & & 0 & & 0 & & & & $\begin{array}{lll}0 & 0\end{array}$ & & 0 & $\begin{array}{ll}0 & 0 \\
0\end{array}$ & & & & & & 500 & & 50 & & & $\begin{array}{lll}0 & 0 & 0\end{array}$ & & 0 & & & & & 0 \\
\hline 5 & 0 & & 5 & & & & & 037 & & 05 & & & & 50 & & & & & 050 & & 050 & & & & 00 & & 0 & & & 050 & & 50 & & $\begin{array}{ll}0 & 0\end{array}$ & 026 & 0 & 50 \\
\hline 6 & 50 & 0 & 45 & 00 & 44 & & & 013 & 0 & & & & $\begin{array}{ll}0 & 0\end{array}$ & 0 & & 0 & & & $\begin{array}{ll}0 & 0\end{array}$ & 0 & 0 & & & & $\begin{array}{lll}0 & 0 & 1\end{array}$ & $\begin{array}{ll}0 & 0\end{array}$ & 0 & & & $\begin{array}{lll}0 & 0 & 0\end{array}$ & & & & $\begin{array}{ll}0 & 0\end{array}$ & $\begin{array}{ll}024 & 0\end{array}$ & $\begin{array}{lll}0 & 0 \\
0\end{array}$ & \\
\hline 7 & 0 & $\begin{array}{ll}0 & 0 \\
\end{array}$ & $\begin{array}{lll}0 & 0 \\
\end{array}$ & $\begin{array}{lll}0 & 0 \\
\end{array}$ & 0 & 0 & 0 & $\begin{array}{ll}0 & 0\end{array}$ & 0 & 0 & 0 & $0 \mathrm{c}$ & $\begin{array}{ll}0 & 0\end{array}$ & 0 & 00 & $\begin{array}{ll}0 & 0\end{array}$ & 0 & 0 & $\begin{array}{ll}0 & 0\end{array}$ & 00 & 0 & 0 & $\begin{array}{lll}0 & 0\end{array}$ & $\begin{array}{ll}0 & 0\end{array}$ & $\begin{array}{lll}0 & 0 & 1\end{array}$ & $\begin{array}{ll}0 & 0\end{array}$ & 0 & 0 & $\begin{array}{ll}0 & 0\end{array}$ & $\begin{array}{lll}0 & 0 & 0\end{array}$ & 0 & 0 & 0 & $\begin{array}{ll}0 & 0\end{array}$ & $\begin{array}{lll}0 & 0 & 0\end{array}$ & $\begin{array}{ll}0 & 0\end{array}$ & $0 \overline{\mathrm{ANS}}$ \\
\hline & 1 & $\begin{array}{ll}1 & 2 \\
1\end{array}$ & 22 & 12 & 22 & 2 & 2 & 22 & 2 & 2 & & 11 & 11 & 1 & 1 & 1 & & 1 & 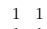 & 11 & 1 & & 1 & 11 & 11 & $\begin{array}{ll}1 & 1\end{array}$ & 1 & 1 & $\begin{array}{ll}22 \\
2\end{array}$ & $\begin{array}{lll}2 & 1 & 2\end{array}$ & 22 & 1 & 2 & $\begin{array}{ll}2 & 2\end{array}$ & $\begin{array}{lll}2 & 2 & 1\end{array}$ & $\begin{array}{ll}11 \\
1\end{array}$ & \\
\hline & 1 & 1 & 11 & 11 & 1 & & 1 & $\begin{array}{ll}1 & 1\end{array}$ & 1 & & & 1 & 11 & 1 & 11 & 1 & 1 & 1 & $\begin{array}{ll}1 & 1\end{array}$ & 11 & & & 1 & 1 & 11 & $\begin{array}{ll}1 & 1\end{array}$ & 1 & 1 & $\begin{array}{ll}1 & 1\end{array}$ & $\begin{array}{lll}1 & 1 & 1\end{array}$ & 11 & 1 & 1 & $\begin{array}{ll}12 \\
2\end{array}$ & $\begin{array}{lll}2 & 1 & 1\end{array}$ & $\begin{array}{ll}11 & 1\end{array}$ & \\
\hline
\end{tabular}


Table F.22: Analysis of dominant solution from Latin Hypercube Sampling method using 900 scenarios

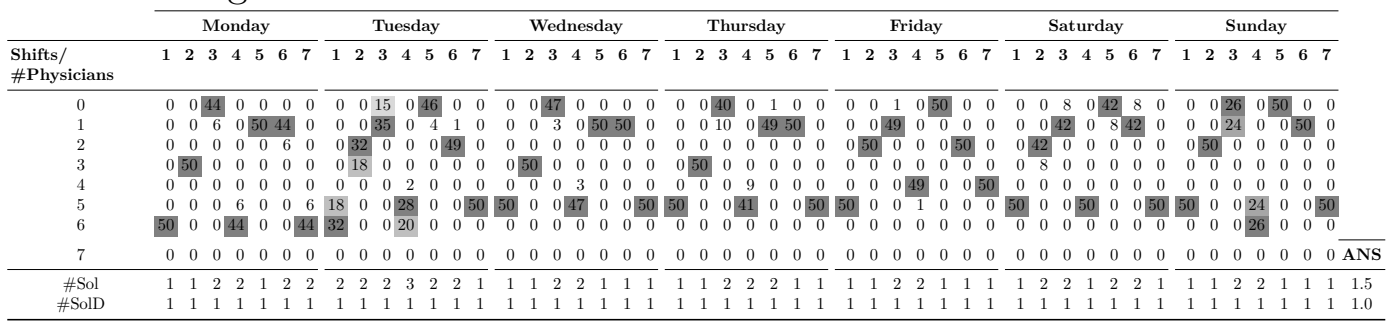

Table F.23: Analysis of dominant solution from Latin Hypercube Sampling method using 1000 scenarios

\begin{tabular}{|c|c|c|c|c|c|c|c|c|c|c|c|c|c|c|c|c|c|c|c|c|c|c|c|c|c|c|c|c|c|c|c|c|c|c|c|c|c|c|c|c|}
\hline \multirow{2}{*}{$\begin{array}{l}\text { Shifts/ } \\
\text { \#Physicians }\end{array}$} & \multicolumn{6}{|c|}{ Monday } & \multicolumn{5}{|c|}{ Tuesday } & \multicolumn{6}{|c|}{ Wednesday } & \multicolumn{5}{|c|}{ Thursday } & \multicolumn{6}{|c|}{ Friday } & \multicolumn{5}{|c|}{ Saturday } & \multicolumn{6}{|c|}{ Sunday } & \\
\hline & 12 & \multicolumn{2}{|c|}{$\begin{array}{ll}2 & 3 \\
\end{array}$} & \multicolumn{2}{|c|}{$\begin{array}{lll}4 & 5 & 6\end{array}$} & & 12 & \multicolumn{2}{|c|}{$\begin{array}{lll}2 & 3 & 4\end{array}$} & & 1 & 2 & \multirow{2}{*}{\multicolumn{2}{|c|}{$\begin{array}{lllll}2 & 3 & 4 & 5\end{array}$}} & \multicolumn{2}{|r|}{7} & 1 & 12 & 34 & & & & & & & & & & & & & & & 1 & & 45 & & & \\
\hline 0 & 0 & 0 & 48 & 01 & 0 & & 00 & 12 & 0 & 49 & 0 & 0 & 0 & & & 0 & & 0 & 047 & 70 & 0 & 0 & & $\begin{array}{ll}0 & 0\end{array}$ & 0 & 050 & 00 & 50 & & 0 & $\begin{array}{lll}6 & 0 \\
\end{array}$ & 44 & $\begin{array}{ll}6 & 0 \\
\end{array}$ & & $\begin{array}{lll}0 & 02 & 2\end{array}$ & 22 & 050 & 0 & & \\
\hline 1 & 0 & 0 & 20 & 49 & 17 & & 00 & 37 & 0 & & 0 & & 0 & 30 & 050 & 50 & 0 & 0 & 0 & $\begin{array}{ll}3 & 0 \\
2 & \end{array}$ & 50 & 50 & & & 50 & 00 & $\begin{array}{ll}0 & 0\end{array}$ & 0 & & 04 & $\begin{array}{ll}44 & 0\end{array}$ & 64 & 440 & & $\begin{array}{lll}0 & 02 \\
2\end{array}$ & 28 & $\begin{array}{ll}0 & 0 \\
\end{array}$ & 49 & 0 & \\
\hline 2 & 0 & 0 & 0 & 0 & 3 & & 033 & 31 & 0 & 05 & & & 0 & 00 & $\begin{array}{ll}0 & 0\end{array}$ & 0 & 0 & 0 & 0 & $\begin{array}{ll}0 & 0 \\
\end{array}$ & 0 & 0 & & 050 & 0 & 00 & 050 & 0 & & 44 & $\begin{array}{lll}0 & 0\end{array}$ & 0 & $\begin{array}{ll}0 & 0\end{array}$ & & 050 & 0 & $\begin{array}{ll}0 & 0\end{array}$ & 1 & 0 & \\
\hline 3 & 05 & 50 & 00 & 0 & 0 & & 017 & 70 & 0 & 0 & 00 & & 50 & 00 & $\begin{array}{ll}0 & 0\end{array}$ & 0 & 0 & 05 & 50 & $\begin{array}{ll}0 & 0 \\
-1\end{array}$ & 0 & 0 & & $\begin{array}{ll}0 & 0\end{array}$ & 0 & 00 & $\begin{array}{ll}0 & 0\end{array}$ & 0 & & 6 & $\begin{array}{ll}0 & 0\end{array}$ & 0 & $\begin{array}{ll}0 & 0\end{array}$ & & $\begin{array}{ll}0 & 0\end{array}$ & 0 & $\begin{array}{ll}0 & 0\end{array}$ & & 0 & \\
\hline 4 & 0 & 0 & 0 & $\begin{array}{ll}0 & 0\end{array}$ & 0 & & 00 & & 1 & 0 & 0 & & 0 & 03 & 30 & 0 & 0 & 0 & & $\begin{array}{ll}03 \\
\end{array}$ & 0 & 0 & & $\begin{array}{ll}0 & 0\end{array}$ & & 490 & $\begin{array}{ll}0 & 0\end{array}$ & 50 & & & $\begin{array}{ll}0 & 0\end{array}$ & 0 & $\begin{array}{ll}0 & 0\end{array}$ & & $\begin{array}{lll}0 & 0\end{array}$ & 0 & $\begin{array}{ll}0 & 0\end{array}$ & & 1 & \\
\hline 5 & 0 & 0 & 02 & 20 & 0 & & 70 & & 35 & & 50 & & 0 & $0 \longdiv { 4 7 }$ & 70 & 0 & 50 & 50 & 0 & $0 \longdiv { 4 7 }$ & 0 & 05 & & 00 & & 10 & $\begin{array}{ll}0 & 0\end{array}$ & 0 & 50 & 0 & 050 & 0 & 050 & & & 02 & 280 & 0 & 49 & \\
\hline 6 & 50 & 0 & 047 & 70 & 04 & & 30 & & 14 & 0 & 0 & 0 & 0 & 00 & $\begin{array}{lll}0 & 0\end{array}$ & 0 & 0 & 0 & 0 & $\begin{array}{ll}0 & 0\end{array}$ & 0 & 0 & & $\begin{array}{lll}0 & 0\end{array}$ & 0 & $\begin{array}{ll}0 & 0\end{array}$ & $\begin{array}{ll}0 & 0\end{array}$ & 0 & 0 & 0 & $\begin{array}{ll}0 & 0\end{array}$ & 0 & $\begin{array}{lll}0 & 0\end{array}$ & & $\begin{array}{lll}0 & 0\end{array}$ & 02 & 220 & & 0 & \\
\hline 7 & 0 & 0 & 0 & 10 & 0 & & 00 & 0 & 0 & 0 & 0 & 0 & 0 & 00 & $\begin{array}{ll}0 & 0\end{array}$ & 0 & 0 & 0 & 0 & $\begin{array}{lll}0 & 0\end{array}$ & 0 & 0 & 0 & $\begin{array}{ll}0 & 0\end{array}$ & $\begin{array}{ll}0 & 0\end{array}$ & 0 & $\begin{array}{ll}0 & 0\end{array}$ & 0 & 0 & 0 & $\begin{array}{ll}0 & 0\end{array}$ & 0 & $\begin{array}{ll}0 & 0\end{array}$ & & $\begin{array}{lll}0 & 0\end{array}$ & 0 & $\begin{array}{ll}0 & 0\end{array}$ & 0 & & ANS \\
\hline \#Sol & 1 & & 23 & 32 & 2 & & 22 & 23 & 3 & 2 & 11 & & 1 & 22 & $\begin{array}{lll}2 & 1\end{array}$ & 1 & 1 & 1 & 1 & 22 & 1 & 1 & & $\begin{array}{ll}11 & 1\end{array}$ & 11 & 2 & 11 & 11 & 1 & 2 & 21 & 2 & $\begin{array}{lll}2 & 1\end{array}$ & & $\begin{array}{lll}11 \\
\end{array}$ & 2 & $\begin{array}{lll}2 & 1\end{array}$ & 2 & 2 & \\
\hline \#SolD & 1 & . & 1 & 1 & 1 & & 11 & 1 & 1 & & 1 & & 1 & 11 & $\begin{array}{ll}1 & 1\end{array}$ & 1 & 1 & 1 & 1 & 11 & & & 1 & 1 & $\begin{array}{ll}1 & 1\end{array}$ & 1 & $\begin{array}{ll}1 & 1\end{array}$ & 11 & 1 & 1 & $\begin{array}{ll}1 & 1\end{array}$ & 1 & $\begin{array}{lll}1 & 1\end{array}$ & & $\begin{array}{lll}11 & 1\end{array}$ & 1 & $\begin{array}{lll}11 & 1\end{array}$ & 1 & 1 & 1.0 \\
\hline
\end{tabular}




\section{G \\ Numerical results from the Hospital A's case study using historical data}

In this Appendix, we present a preliminary study in the Hospital A using just historical data as scenarios in the optimization model.

Based on historical patient arrival data, we created possible realization scenarios that were used to construct a finite set $S C$. The dataset to which we had access consisted of 68 weeks, each of which was taken as an individual scenario. Hence, 68 demand scenarios were generated, each of which corresponded to the realization of real ED historical demand and was associated with a value of parameter $D E M_{h t}^{c}$. The optimal solution for the model was achieved. The computational characteristics of the model are presented in Table G.1.

Table G.1: Computational results to case study in the Hospital A

\begin{tabular}{lr}
\hline Number of scenarios & 68 \\
Number of constraints & 47,84 \\
Number of variables & 32,621 \\
Number of non zeros & 309,401 \\
Number of iterations & $2,150,151$ \\
CPU time (sec) & 436.93 \\
\hline
\end{tabular}

At this point, it is worth pointing out that the current manually-defined ED schedule underutilizes workload capacity due to the difficulty of manually searching for feasible schedule solutions. Total available physician workload (tallied from each physician's available workload) amounts to 4,200 hours, but only 3,522 hours are currently allocated in the manual schedule. More importantly, personnel costs account for 3,888 physician work hours, but approximately $10 \%$ of such costs cover wasted, i.e., unscheduled work hours. However, it is reasonable to expect that our model's optimal solution would make use of full workload capacity, which could misleadingly seem to indicate an increase in total physician workload when compared to the current schedule. To ensure fairness of comparison between the current schedule and the one generated by our optimization model, we report two sets of results: the first set was generated considering the current overall staffing level (44 physicians) and limiting the total workload in the model by the same number of hours currently used in the ED ; the second set was generated considering the current 
overall staffing level (44 physicians) without limiting the total workload in the model by the same number of hours currently used in the ED. In what follows, we will refer to the former as being generated by Model 1 and the latter as being generated by Model 2. For the first set of results, we include in the model presented in Section 4.2 the constraint as follow:

$$
\sum_{d} \sum_{s} \sum_{p} x_{s d p} D U R_{s} \leq M A X \_W L
$$

Constraint (G-1) enforces that the total number of hours assigned in the entire planning horizon is less than or equal to the total allowable workload.

Being $M A X \_W L$ the maximum workload that can be allocated for each planning horizon $p$. For the Model $1 M A X_{-} W L=3,522$ hours and for the Model 2 MAX_WL $=3,888$ hours

We begin by discussing the results generated by Model 1. Table G.2 contrasts indicators of service quality for the current schedule with those obtained through discrete-event simulation of the optimal schedule. In addition to the value of each indicator, we also report on half-width (HW), when applicable. The half-width value may be interpreted by saying "in $95 \%$ of repeated trials, the sample mean would be reported as within the interval sample mean \pm half-width". In the last column of Table G.2 $\left(\Delta_{1} \%\right)$, we present the percentage variation of the indicator values. A number of points are worthy of mention: first, note the reduction of approximately $40 \%$ in the frequency of queues and an even greater reduction (of the order of $78 \%$ ) in the frequency of queues with more than 10 patients (when comparing the schedule from Model 1 with the current schedule). Moreover, the schedule from Model 1 leads to a reduction of approximately $73 \%$ in the number of patients in queue. The decrease in queue occurrence and the number of patients in the queue is reflected in the reduction of the average waiting time and, consequently, in the time door-to-doctor (since the average service time is the same for both the current schedule and the one generated by Model 1). The average time doorto-doctor decreased by approximately $73 \%$ in relation to the current schedule. Finally, note that the total number of physician hours used by the schedule from Model 1 is inferior to the total used in the current schedule. This occurs due to constraint (G-1), which was added to ensure a fair comparison between the current and model-generated schedules, for reasons discussed above.

We then used historical data to compare service capacity with demand. Of note, the schedule generated by Model 1 provides greater adherence of service capacity to varying demand, i.e., more physicians are on duty on days with greater demand than on other days. For example, we verified that on Mondays, which is the day of highest demand, more physicians are available 
Table G.2: Discrete-event simulation results for Model 1

\begin{tabular}{lrrrrr} 
& \multicolumn{2}{c}{ Current schedule } & \multicolumn{2}{c}{ Model 1 } \\
\cline { 2 - 6 } & Value & HW & Value & HW & $\Delta_{1} \%$ \\
\hline Number of patients & $85,623.66$ & 78.28 & $85,611.4$ & 73.84 & $-0.01 \%$ \\
Frequency of queue (\%) & 24.05 & - & 14.37 & $--40.25 \%$ \\
Frequency of queue $>10$ patients (\%) & 9.84 & - & 2.11 & - & $-78.58 \%$ \\
Frequency of queue $\leq 10$ patients (\%) & 14.21 & - & 12.26 & $--13.72 \%$ \\
Average number of patients in queue & 3.00 & 0.08 & $0.79<0.02$ & $-73.67 \%$ \\
Average time door-to-doctor (min) & 24.53 & 0.63 & $6.48<0.14$ & $-73.61 \%$ \\
Total number of physician hours used & $3,522.00$ & $-3,504.00$ & - & $-0.51 \%$ \\
\hline
\end{tabular}

on CM than on any other day of the week. Figure G.1 presents the histograms of idle capacity and queue frequency for the current schedule and the schedule generated by Model 1, where negative values indicate idle capacity and positive values indicate unmet demand, resulting in the queue. Thus, for example, the value -18 means that there was a capacity to see 18 additional patients in a given work hour, which implies that there were 6 physicians in excess in CM, considering each physician can visit 3 patients per hour. 


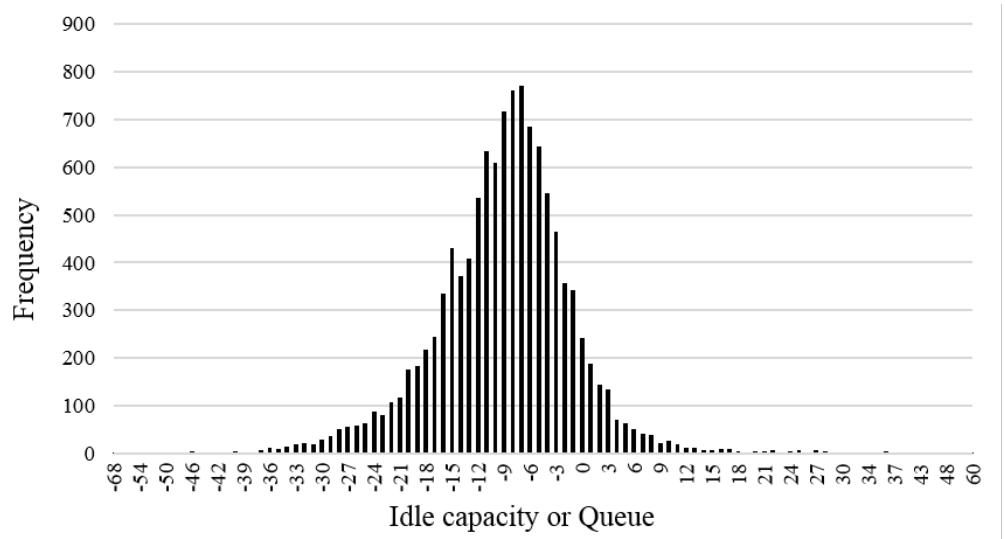

(a)

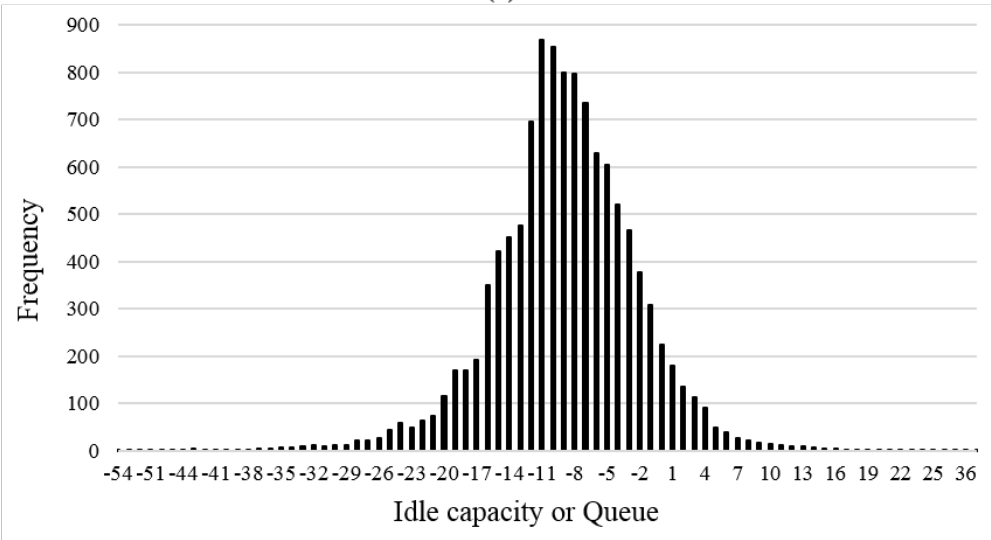

(b)

Figure G.1: Histogram of frequency distribution of idle capacity (negative values) or queue (positive values) for (a) current schedule; (b) schedule generated by Model 1

As shown in Figure G.1(a), idleness occurs more frequently than queues in the current schedule, but the number of people in queue can be very large, up to 60 . On the other hand, in the histogram in Figure G.1(b) we observe that queues are not only less frequent for the schedule generated by Model 1, but also smaller.

We now proceed by discussing the results generated by Model 2. Recall that Model 2 does not include constraint (G-1), meaning that the total workload is not limited by the total number of work hours currently used in the manually-defined schedule. Table G.3 contrasts indicators of service quality for the current schedule with those obtained through discrete-event simulation of the optimal schedule generated by Model 2. In addition to the value of each indicator, we also report on half-width (HW), when applicable. In the last column of Table G.3 $\left(\Delta_{2} \%\right)$, we present the percentage variation of the indicator values. Several points are worthy of mention: first, note the reduction of approximately $62 \%$ in the frequency of queues, which is a significantly 
greater reduction than the one shown in Table G.2. Moreover, the frequency of queues with more than 10 patients (one of the main quality indicators defined by managers from the real ED we are analyzing) is approximately $0.91 \%$, which is considerably lower than both the current schedule and the schedule generated by Model 1. Additionally, the schedule from Model 2 leads to a reduction of approximately $86 \%$ in the number of patients in the queue, while the average time door-to-doctor decrease by approximately $86 \%$ in relation to the current schedule. Taken together, these results indicate that the schedule generated by Model 2 takes better advantage of the total available workload during the planning horizon and more efficiently allocates physicians when compared to both the current schedule and the schedule generated by Model 1. A direct comparison of Tables G.2 and G.3 reveals that all queue and wait time indicators are considerably lower for Model 2 than for Model 1 or the current schedule.

Table G.3: Discrete-event simulation results for Model 2

\begin{tabular}{lrrrrr} 
& \multicolumn{2}{c}{ Current schedule } & \multicolumn{2}{c}{ Model 2 } \\
\cline { 2 - 6 } & Value & HW & Value & HW & $\Delta_{2} \%$ \\
\hline Number of patients & $85,623.66$ & 78.28 & $85,622.92$ & 70.94 & $0.00 \%$ \\
Frequency of queue $(\%)$ & 24.05 & - & 9.00 & - & $-62.58 \%$ \\
Frequency of queue $>10$ patients (\%) & 9.84 & - & 0.91 & - & $-90.71 \%$ \\
Frequency of queue $\leq 10$ patients (\%) & 14.21 & - & 8.08 & - & $-43.14 \%$ \\
Average number of patients in queue & 3.00 & 0.08 & 0.42 & $<0.01$ & $-86.11 \%$ \\
Average time door-to-doctor (min) & 24.53 & 0.63 & $3.41<0.10$ & $-86.12 \%$ \\
Total number of physician hours used & $3,522.00$ & - & $3,888.00$ & - & $9.41 \%$ \\
\hline
\end{tabular}

We end with a discussion on the use of the total physician workload in the schedule from Model 1 vs. the one from Model 2. Since we do not limit the total workload in Model 2, its use is greater than in Model 1. This means that Model 2 outperforms Model 1 (and, consequently, the manual schedule) in the scheduling phase. We can further observe that the average utilization is lower in the schedule generated by Model 2, indicating that physician idleness is greater. This occurs because, by removing constraint (G-1), the model can further minimize queues by allocating more physicians than the minimum required, thus leading to idleness at certain points in time. Of note, Model 2 more adequately accounts for real ED schedule requirements given that it does not include the artificially-defined constraint on total physician workload. 


\section{H \\ Size of the model from the Hospital B's case study from Chapter 4}

The size of the models (i.e., variables and constraints), average execution times, and their standard deviation are shown in Table H.1.

Table H.1: Size of the model from the case study in the Hospital B

\begin{tabular}{ccccc}
\hline Scenarios & Variables & Constraints & Time(s) & St.Dev (s) \\
\hline $\mathbf{1 0 0}$ & 50443 & 307516 & 172.99 & 108.72 \\
$\mathbf{2 0 0}$ & 84043 & 324316 & 165.04 & 40.56 \\
$\mathbf{3 0 0}$ & 117643 & 341116 & 278.58 & 194.72 \\
$\mathbf{4 0 0}$ & 151243 & 357916 & 282.36 & 36.16 \\
$\mathbf{5 0 0}$ & 184843 & 374716 & 508.80 & 344.94 \\
$\mathbf{6 0 0}$ & 218443 & 391516 & 1512.113 & 2578.02 \\
$\mathbf{7 0 0}$ & 252043 & 408316 & 851.34 & 743.56 \\
$\mathbf{8 0 0}$ & 285643 & 425116 & 1735.79 & 1967.06 \\
$\mathbf{9 0 0}$ & 319243 & 441916 & 1932.535 & 841.31 \\
$\mathbf{1 0 0 0}$ & 352843 & 458716 & 2188.666 & 879.34 \\
\hline
\end{tabular}




\section{Extra SAA material from the Hospital B's case study}

Table I.1 shows the minimum and maximum confidence levels for the optimal gap in each experiment.

Table I.1: Confidence interval of the optimal gap from case study in the Hospital B

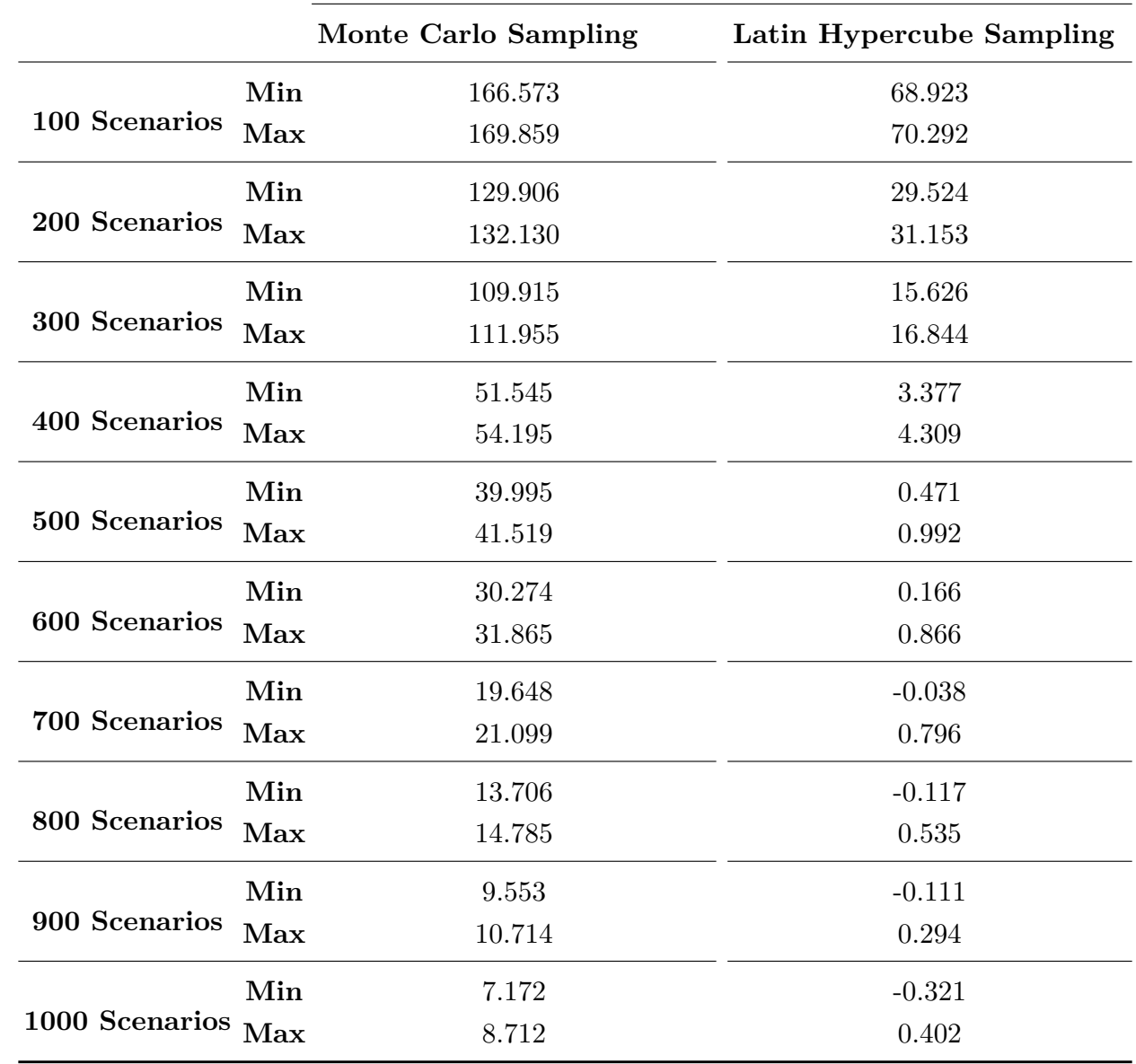

Tables I.2 and I.3 show the number of first-stage solutions in the insample analysis, i.e., how many different solutions were observed in each replication. In the tables, we present the results of each replication by varying the number of scenarios using the Monte Carlo and the Latin Hypercube methods, respectively. The solution we consider for analysis here is the number of physicians allocated for each day and hour. For example, in Table I.2, we see that using 100 scenarios we have XX possible solutions for the number of physicians that should be allocated at Hour 0 on Monday. 
We can observe from Table I.2 that as we increase the number of scenarios, the number of possible solutions decreases, tending to converge to a single solution. We see that the Average Number of Solutions (ANS) is 4.3 for 100 scenarios and decreases progressively reaching 3.0 for 1,000 scenarios. We observed the same behavior in Table I.3 using the Latin Hypercube technique. However, we noticed a faster convergence tending to a single solution using this sampling method (ANS $=2.99$ for 1,000 scenarios). 
Table I.2: Number of solutions using Monte Carlo Sampling method
Monday Tuesday Wednesday Thursday Friday
Saturday Sunday

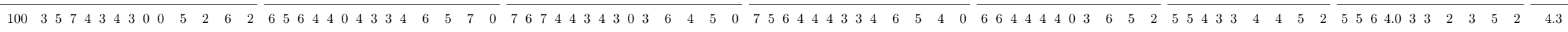

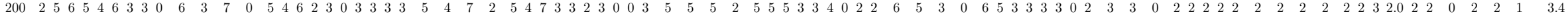

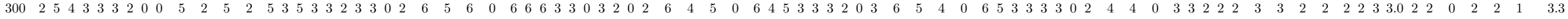

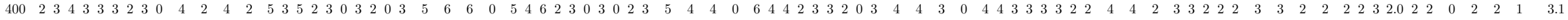

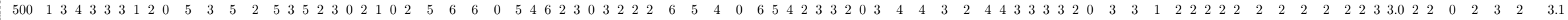

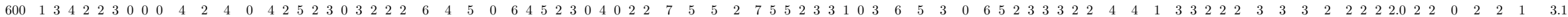

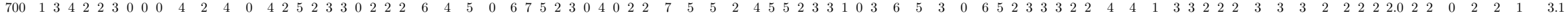

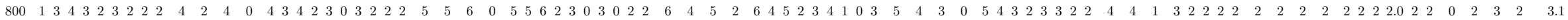

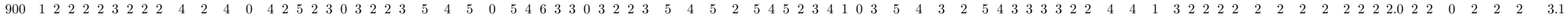

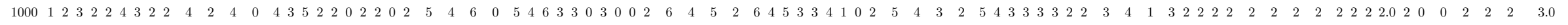

${ }^{\mathrm{a}}$ ANS - Average Number of Solutions

Table I.3: Number of solutions using Latin Hypercube Sampling method

Monday Tuesday Nednesday $\quad$ Thursday $\quad$ Friday $\quad$ Sunday

$\overline{\text { Hour } 0123467812131415} \overline{012346789121314} \overline{012346789121314} \overline{01234678912131415} \overline{01234679121314} \overline{0123412131415} \overline{012341415 \text { ANS }}$

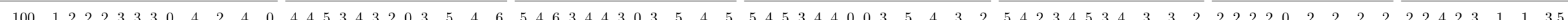

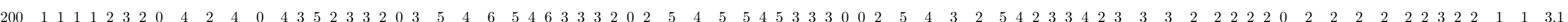

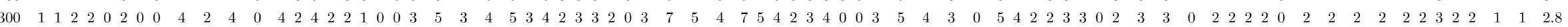

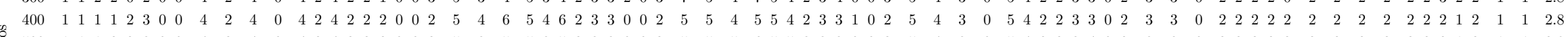

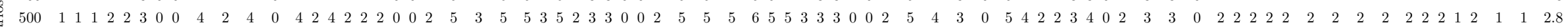

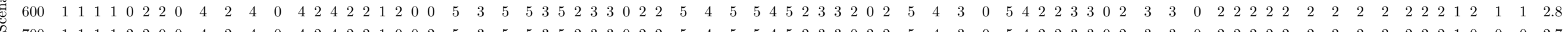

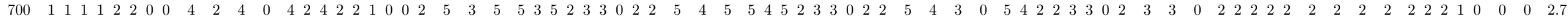

$\begin{array}{lllllllllllllllllllllllllllllllllllllllllllllllllllllllllllllllllllllllllllllllll}800 & 1 & 1 & 1 & 1 & 0 & 2 & 2 & 0 & 4 & 2 & 4 & 2 & 4 & 2 & 5 & 2 & 2 & 1 & 0 & 0 & 2 & 4 & 3 & 5 & 5 & 3 & 5 & 2 & 3 & 3 & 0 & 0 & 2 & 5 & 4 & 5 & 5 & 4 & 4 & 2 & 2 & 3 & 0 & 0 & 2 & 4 & 3 & 3 & 0 & 5 & 4 & 2 & 2 & 3 & 3 & 0 & 2 & 3 & 3 & 0 & 2 & 2 & 2 & 2 & 2 & 2 & 2 & 2 & 2 & 2 & 2 & 2 & 1 & 2 & 1 & 1 & 2.7\end{array}$

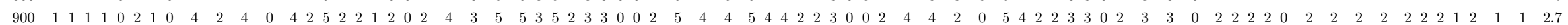

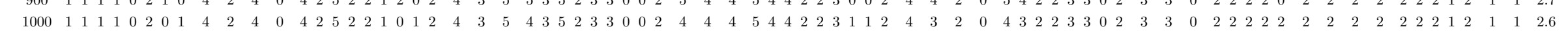

${ }^{a}$ ANS - Average Number of Solutions 
As for each number of scenarios we run 50 replications, it is important to analyze the frequency with which each solution appears. With this, we can analyze dominant solutions. We call dominant solutions the solutions that appear most often. Tables I.4 - I.13 present the results of the dominant solution analysis for the Monte Carlo method. Tables I.14 - I.23 show the results for the Latin Hypercube technique. For dominant solution analysis, we use a color scale according to Figure F.1. When a solution appears 26 times or more (more than $50 \%$ ) we consider only 1 dominant solution, otherwise we consider as dominant solution any solution that appears between 11 and 25 times.

Considering only the dominant solutions, we observed that even with just 100 scenarios we have a low number of possible solutions using both methods. Despite a large number of possible solutions, we see that as we increase the number of scenarios, even if other solutions ever appear, the solution tends to converge to a point, which we call the dominant solution and which can be considered the true solution for the problem. The results showed that the LHS method converges more quickly to a true solution since the ANS for dominant solutions always decreases and from 500 scenarios the ANS is already about 1.09. Using the MCS, we observed a greater oscillation of the ANS. 
Table I.4: Analysis of dominant solution from Monte Carlo Sampling method using 100 scenarios

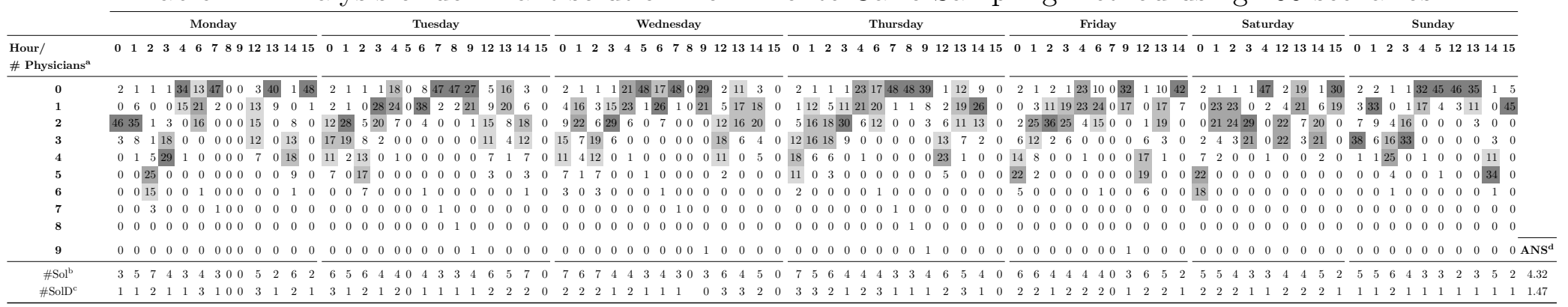

a \#Physicians - Number of Physicians assigned to that shift

\# \#Sol - Number of different solutions

${ }^{c}$ \#SolD - Number of dominant solutions

d ANS - Average Number of Solutions

Table I.5: Analysis of dominant solution from Monte Carlo Sampling method using 200 scenarios

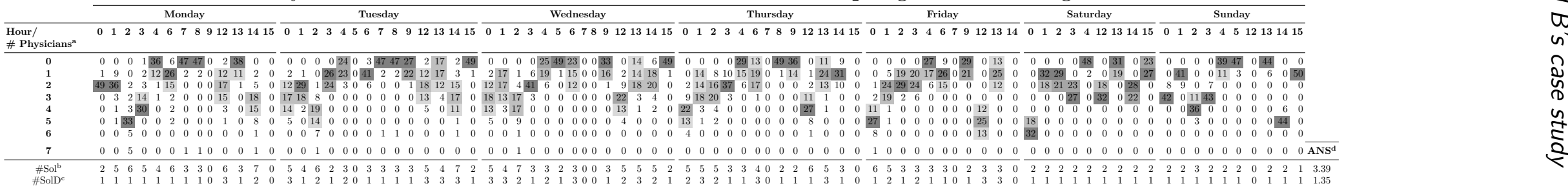


Table I.6: Analysis of dominant solution from Monte Carlo Sampling method using 300 scenarios

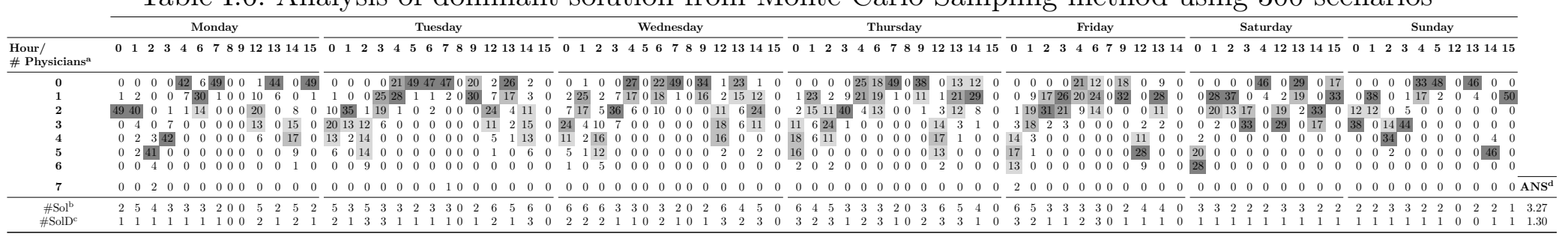


Table I.7: Analysis of dominant solution from Monte Carlo Sampling method using 400 scenarios

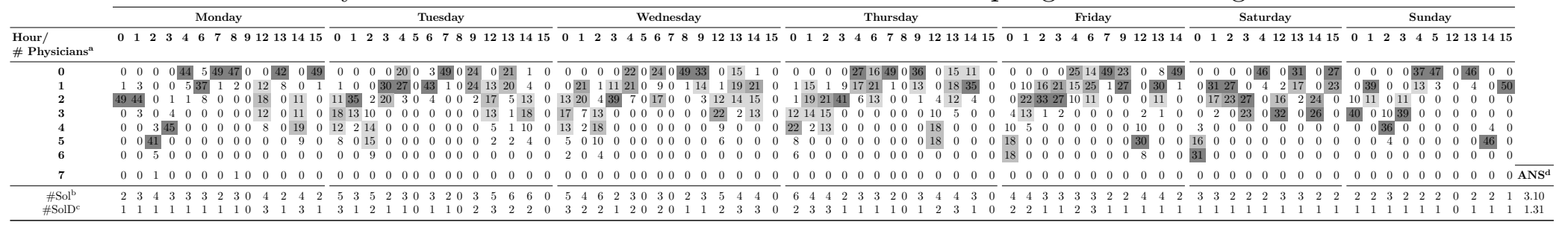


Table I.8: Analysis of dominant solution from Monte Carlo Sampling method using 500 scenarios

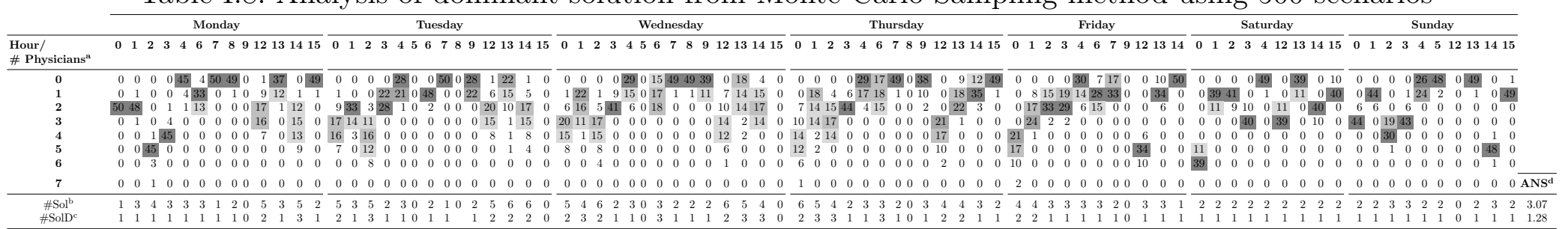


Table I.9: Analysis of dominant solution from Monte Carlo Sampling method using 600 scenarios

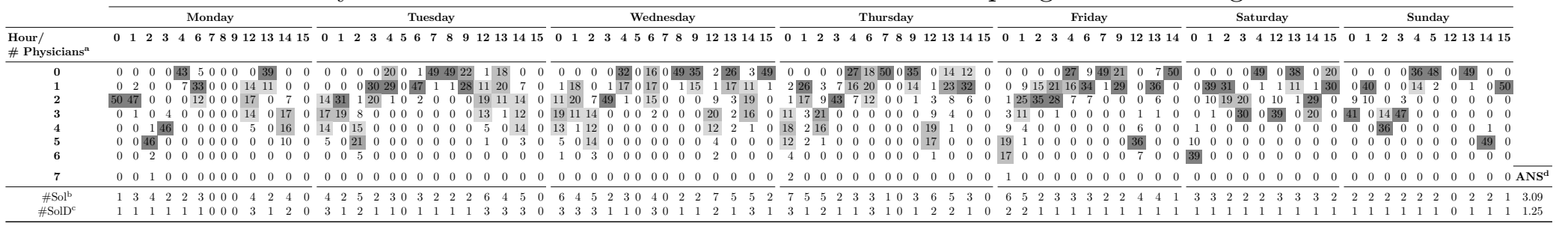


Table I.10: Analysis of dominant solution from Monte Carlo Sampling method using 700 scenarios

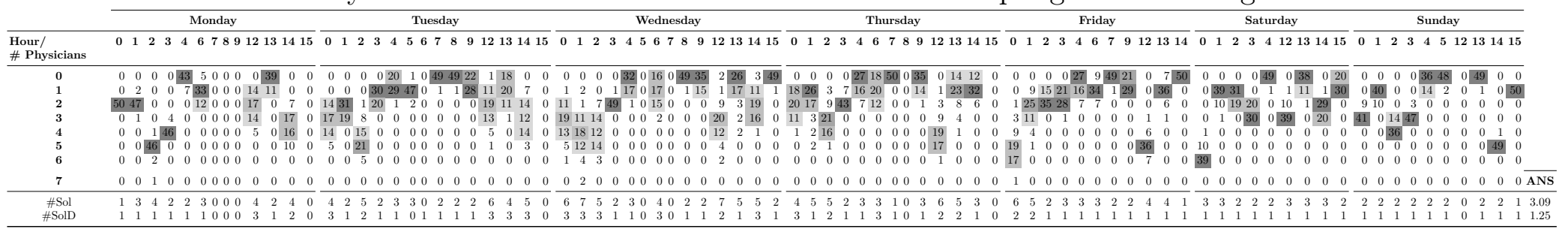


Table I.11: Analysis of dominant solution from Monte Carlo Sampling method using 800 scenarios

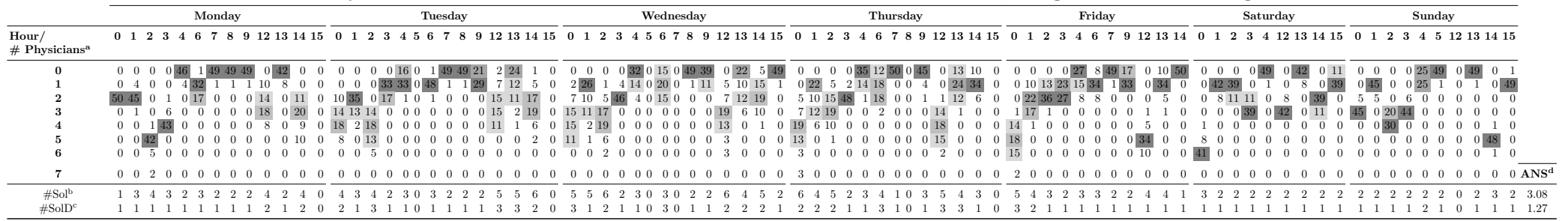


Table I.12: Analysis of dominant solution from Monte Carlo Sampling method using 900 scenarios

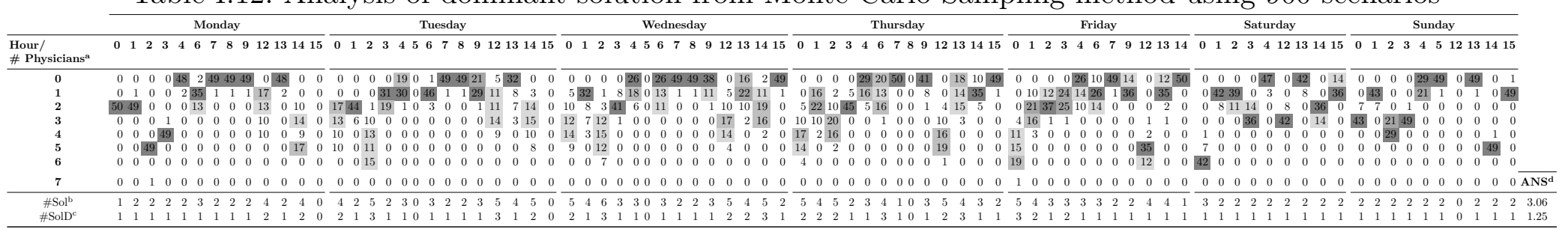

Table I.13: Analysis of dominant solution from Monte Carlo Sampling method using 1000 scenarios

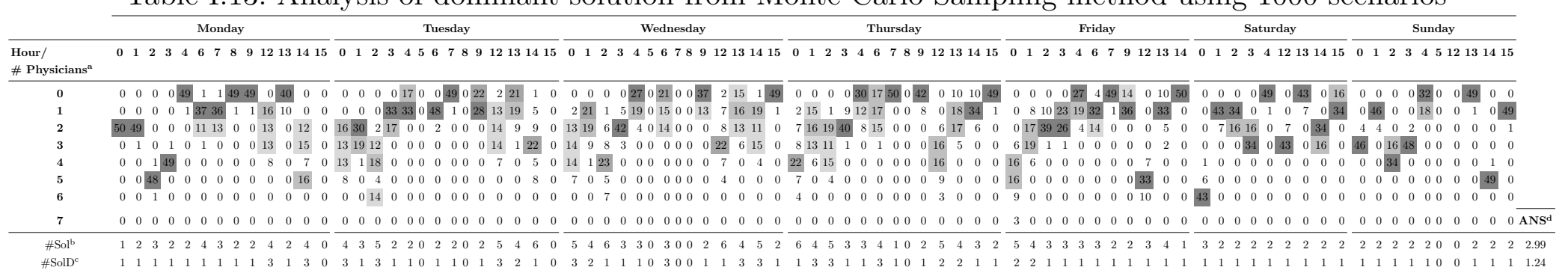


Table I.14: Analysis of dominant solution from Latin Hypercube Sampling method using 100 scenarios

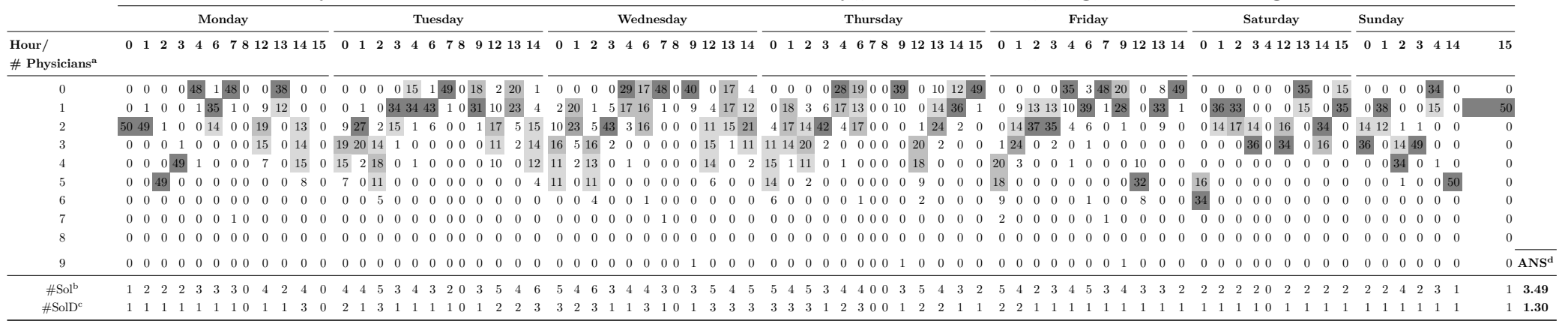

${ }^{\mathrm{a}}$ \#Physicians - Number of Physicians assigned to that shift

b \#Sol - Number of different solutions

${ }^{c}$ \#SolD - Number of dominant solutions

$\mathrm{d}_{\text {ANS - Average Number of Solutions }}$

Table I.15: Analysis of dominant solution from Latin Hypercube Sampling method using 200 scenarios

\begin{tabular}{|c|c|c|c|c|c|c|c|c|}
\hline & Monday & Tuesday & Wednesday & Thursday & Friday & Saturday & Sunday & \\
\hline $\begin{array}{l}\text { ur/ } \\
\text { Physicians }^{\mathrm{a}}\end{array}$ & $\begin{array}{lllllll}0 & 1 & 2 & 3 & 4 & 6 & 7812131415\end{array}$ & $\begin{array}{llllllllll}0 & 1 & 2 & 3 & 4 & 6 & 78 & 9121314\end{array}$ & $\begin{array}{llllllllll}0 & 1 & 2 & 3 & 4 & 6 & 78 & 91213 & 14\end{array}$ & 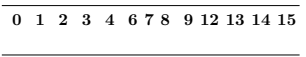 & $\begin{array}{lllllllllll}0 & 1 & 2 & 3 & 4 & 6 & 7 & 9 & 12 & 13 & 14\end{array}$ & 1223412131415 & $\begin{array}{llllll}0 & 1 & 2 & 3 & 4 & 14\end{array}$ & 15 \\
\hline $\begin{array}{l}0 \\
1\end{array}$ & 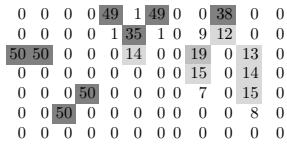 & 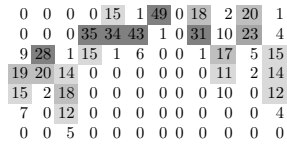 & 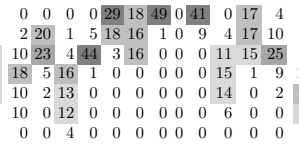 & 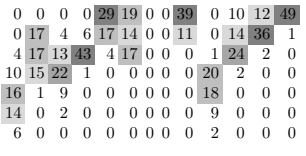 & 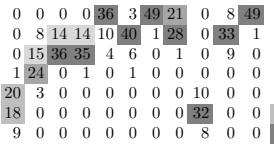 & 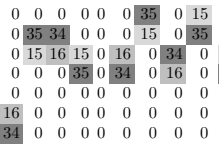 & 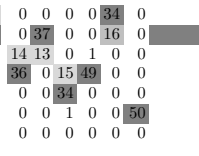 & $\begin{array}{l}0 \\
0 \\
0 \\
0\end{array}$ \\
\hline 7 & $\begin{array}{llllllllllll}0 & 0 & 0 & 0 & 0 & 0 & 0 & 0 & 0 & 0 & 0 & 0 \\
\end{array}$ & $\begin{array}{llllllllllll}0 & 0 & 0 & 0 & 0 & 0 & 0 & 0 & 0 & 0 & 0 & 0 \\
\end{array}$ & $\begin{array}{lllllllllllll}0 & 0 & 0 & 0 & 0 & 0 & 0 & 0 & 0 & 0 & 0 & 0 \\
\end{array}$ & $\begin{array}{llllllllllllll}0 & 0 & 0 & 0 & 0 & 0 & 0 & 0 & 0 & 0 & 0 & 0 \\
\end{array}$ & $\begin{array}{ccccccccccc}2 & 0 & 0 & 0 & 0 & 0 & 0 & 0 & 0 & 0 & 0 \\
\end{array}$ & 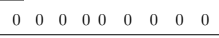 & $\begin{array}{lllllll}0 & 0 & 0 & 0 & 0 & 0 \\
\end{array}$ & $0 \overline{\mathrm{ANS}^{\mathrm{d}}}$ \\
\hline $\begin{array}{l}\# \mathrm{HSol}^{\mathrm{b}} \\
\# \mathrm{SolD}^{c}\end{array}$ & $\begin{array}{llllllllllll}1 & 1 & 1 & 1 & 2 & 3 & 2 & 0 & 4 & 2 & 4 \\
1 & 1 & 1 & 1 & 1 & 1 & 1 & 0 & 2 & 1 & 3 & 0\end{array}$ & 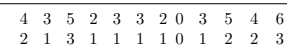 & 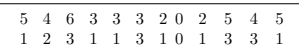 & 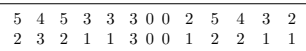 & $\begin{array}{ccccccccccc}5 & 4 & 2 & 3 & 3 & 4 & 2 & 3 & 3 & 3 & 2 \\
2 & 2 & 1 & 1 & 1 & 1 & 1 & 1 & 1 & 1 & 1\end{array}$ & $\begin{array}{lllllllll}2 & 2 & 2 & 2 & 0 & 2 & 2 & 2 & 2 \\
1 & 1 & 1 & 1 & 0 & 1 & 1 & 1 & 1\end{array}$ & $\begin{array}{llllll}2 & 2 & 3 & 2 & 2 & 1 \\
1 & 1 & 1 & 1 & 1 & 1\end{array}$ & $\begin{array}{ll}1 & 3.09 \\
1 & 1.29\end{array}$ \\
\hline
\end{tabular}


Table I.16: Analysis of dominant solution from Latin Hypercube Sampling method using 300 scenarios

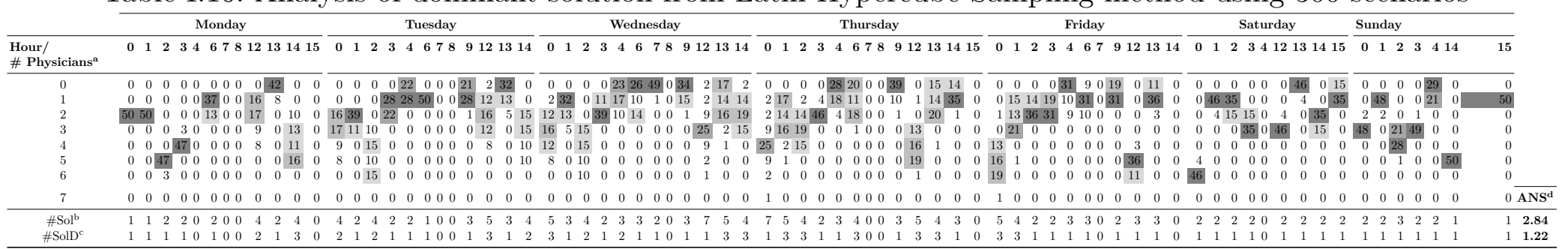


Table I.17: Analysis of dominant solution from Latin Hypercube Sampling method using 400 scenarios

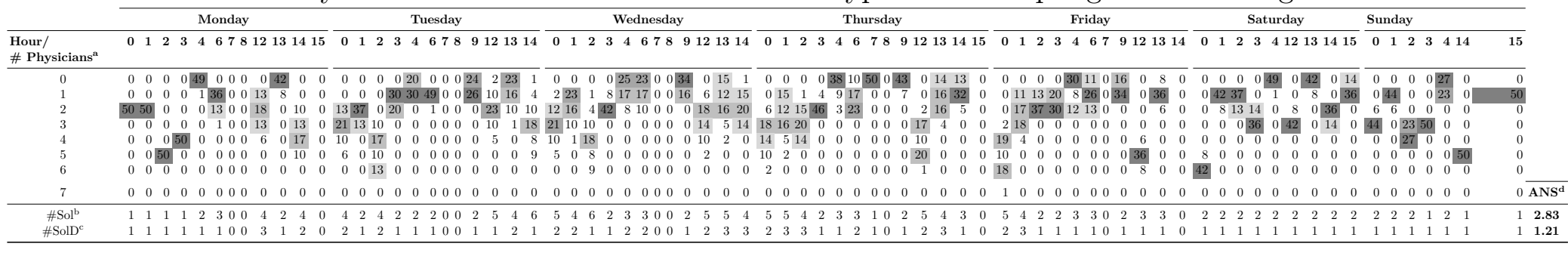


Table I.18: Analysis of dominant solution from Latin Hypercube Sampling method using 500 scenarios

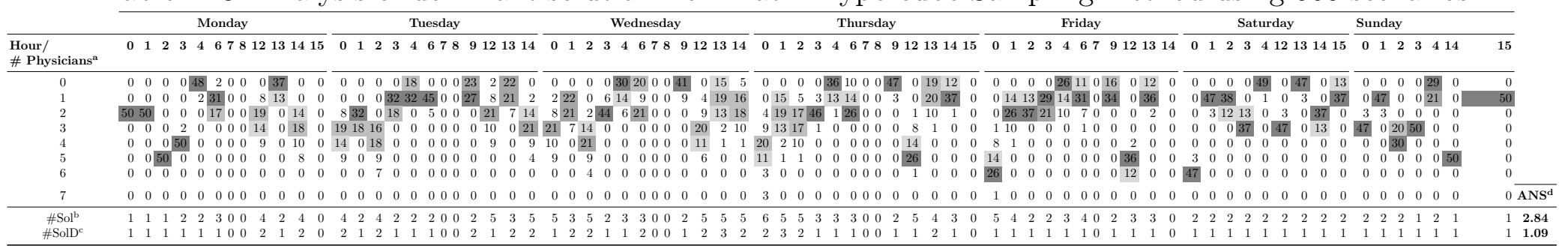


Table I.19: Analysis of dominant solution from Latin Hypercube Sampling method using 600 scenarios

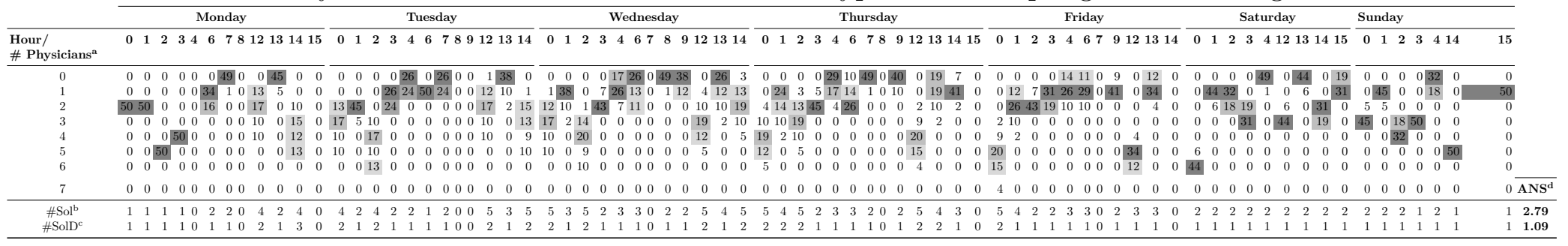


Table I.20: Analysis of dominant solution from Latin Hypercube Sampling method using 700 scenarios

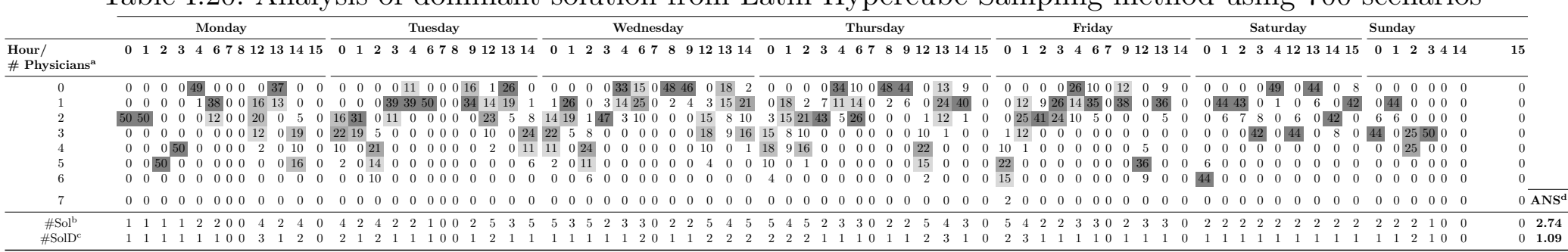


Table I.21: Analysis of dominant solution from Latin Hypercube Sampling method using 800 scenarios

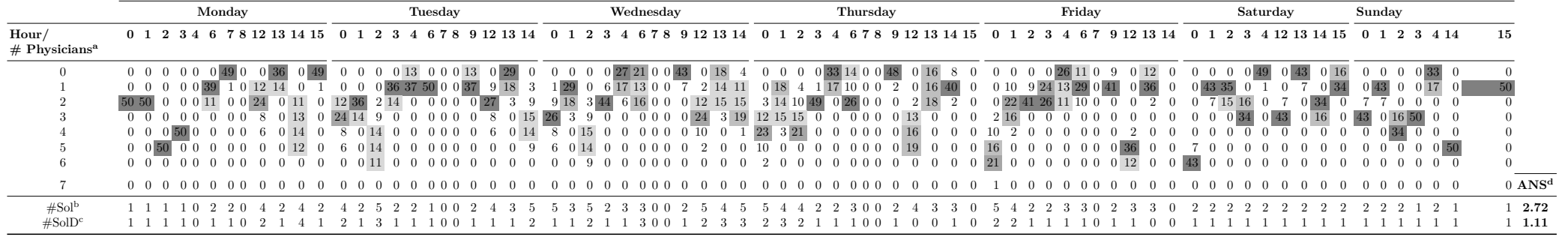


Table I.22: Analysis of dominant solution from Latin Hypercube Sampling method using 900 scenarios

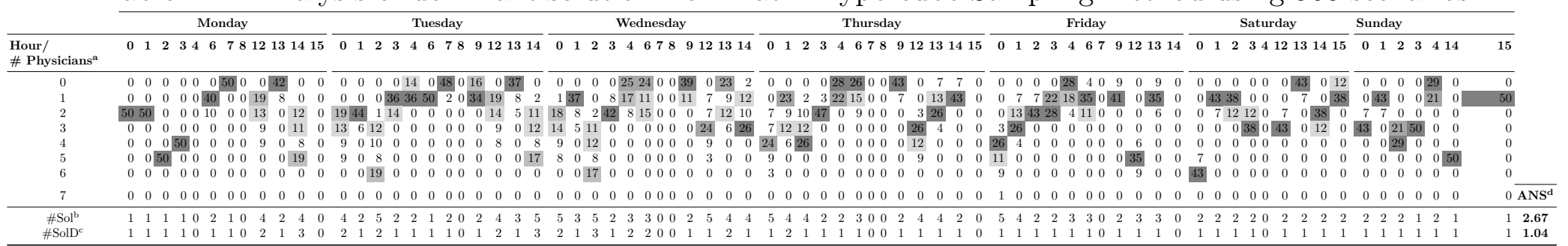

Table I.23: Analysis of dominant solution from Latin Hypercube Sampling method using 1000 scenarios

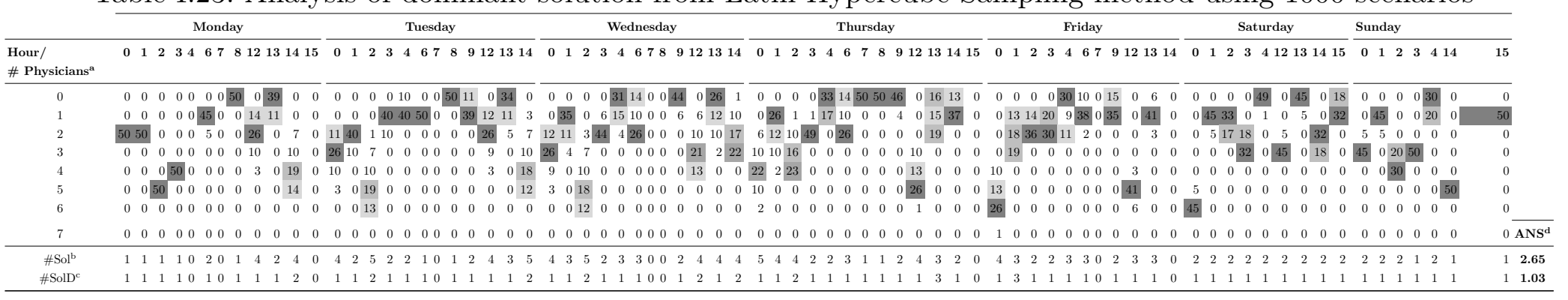




\section{J \\ Numerical results of the Hospital B's case study using histor- ical data}

In this Appendix, we present a preliminary study in the Hospital B using just historical data as scenarios in the optimization model.

We used the historical patient arrival data to create possible realization scenarios that were used to construct a finite set $S C$. The dataset to which we had access consisted of 42 weeks, which each week correspond to an individual scenario each of which corresponded to the realization of real ED historical demand and was associated with a value of parameter $D E M_{h t}^{c}$. The optimal solution for the model was achieved. The computational characteristics of the model are presented in Table J.1.

Table J.1: Computational results to case study in the Hospital B

\begin{tabular}{lr}
\hline Number of scenarios & 42 \\
Number of constraints & 297,605 \\
Number of variables & 30,619 \\
Number of non zeros & 719,867 \\
Number of iterations & 186,706 \\
CPU time (sec) & 94,34 \\
\hline
\end{tabular}

Table J.2 contrasts indicators of service quality for the current schedule with those obtained through discrete-event simulation of the optimal schedule. In addition to the value of each indicator, we also report on half-width (HW), when applicable. The half-width value may be interpreted by saying "in $95 \%$ of repeated trials, the sample mean would be reported as within the interval sample mean \pm half-width". In the last column of Table J.2 $\left(\Delta_{1} \%\right)$, we present the percentage variation of the indicator values. It is worthy of mentioning that: first, the frequency of queue decrease in order of $22 \%$ and the frequency of queue with more than 10 patients about 39\%. Moreover, the schedule from model 1 leads to a reduction of approximately $43 \%$ in the number of patients in queue. Decreasing queue occurrence and the number of patients in the queue, we observed a reduction of the average waiting time and, consequently, in the time door-to-doctor. The average time door-to-doctor decreased by about $32 \%$ in relation to the current schedule. The total number of physician hours 
Appendix J. Numerical results of the Hospital B's case study using historical data

used by the schedule from the model is equal to the total used in the current schedule.

Table J.2: Discrete-event simulation results for case study in the Hospital B

\begin{tabular}{lrrrrr} 
& \multicolumn{2}{c}{ Current schedule } & \multicolumn{3}{c}{ Model } \\
\cline { 2 - 6 } & Value & HW & Value & HW & $\Delta_{1} \%$ \\
\hline Number of patients & $72,798.72$ & 60.18 & $72,981.01$ & 63.36 & $0.25 \%$ \\
Frequency of queue (\%) & 32.21 & - & 25.25 & - & $-21.61 \%$ \\
Frequency of queue $>$ 10 patients (\%) & 12.75 & - & 7.83 & $-38.59 \%$ \\
Frequency of queue $\leq$ 10 patients (\%) & 19.46 & - & 17.69 & - & $-9.10 \%$ \\
Average number of patients in queue & 3.87 & 0.45 & $2.23<0.04$ & $-42.45 \%$ \\
Average time door-to-doctor (min) & 36.55 & 0.45 & 25.02 & $<0.47$ & $-31.55 \%$ \\
Total number of physician hours used & $1,457.00$ & - & $1,457.00$ & - & $0.00 \%$ \\
\hline
\end{tabular}

Figure J.1 presents the histograms of idle capacity and queue frequency for the current schedule and the schedule generated by the model. As shown in Figure J.1(a), idleness occurs more frequently than queue in the current schedule, but the number of people in queue can be very large, up to 72 . On the other hand, the histogram in Figure J.1(b) has a smaller right tail distribution than the one in Figure J.1(a), indicating that queues are not only less frequent for the schedule generated by the model, but also smaller. 
Appendix J. Numerical results of the Hospital B's case study using historical data

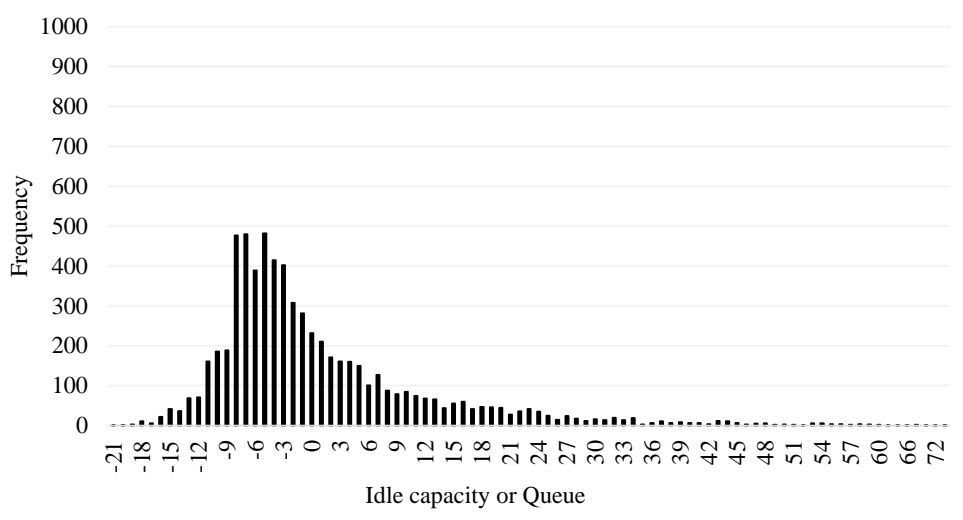

(a)

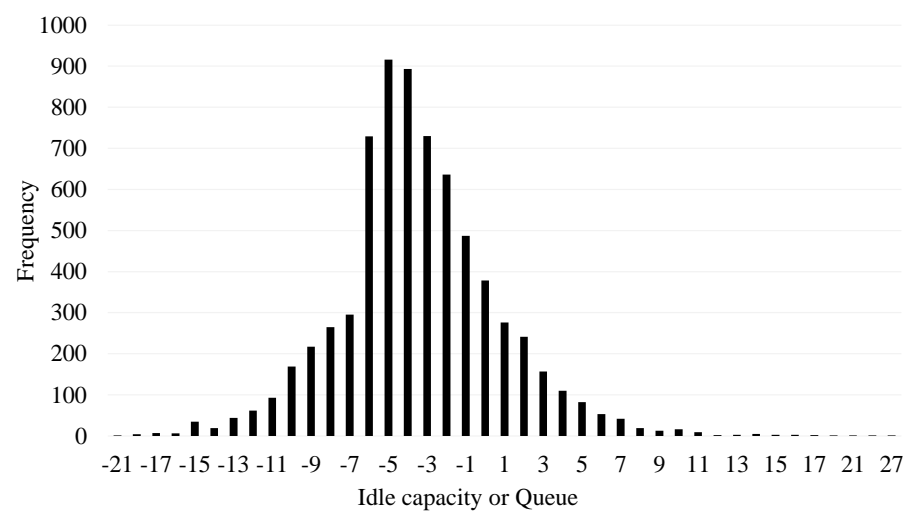

(b)

Figure J.1: Histogram of frequency distribution of idle capacity (negative values) or queue (positive values) for (a) current schedule; (b) schedule generated by Model 1

From the results, we noted that for this case study it was also possible to achieve a significant reduction in all queue and waiting time indicators, just making the doctors' entry time in the ED studied more flexible. 
$\mathrm{K}$

Extra material from the Hospital B's case study from Chapter 4

Table K.1: Current schedule of physicians in the Hospital B

\begin{tabular}{|c|c|c|c|c|c|c|c|c|c|c|c|}
\hline \multirow{2}{*}{$\overline{\text { Shift }}$} & \multicolumn{5}{|c|}{ Fixed Physicians } & \multicolumn{6}{|c|}{ On-callers Physicians } \\
\hline & M & $\mathbf{T}$ & $\mathbf{W}$ & & F S S & M & $\mathbf{T}$ & W & & F S & $\mathbf{S}$ \\
\hline 1 & & & 1 & 1 & 2 & & & & & & \\
\hline 2 & & 1 & 1 & & & & & & & & \\
\hline 3 & 2 & 1 & 1 & & & 7 & 5 & 6 & 6 & 68 & 9 \\
\hline 4 & & 1 & & & & & & & & & \\
\hline 5 & 1 & 1 & 2 & & 1 & & & & & & \\
\hline 6 & 2 & 1 & & 2 & & & & & & & \\
\hline 7 & & & & & 11 & & & & & & \\
\hline 8 & 1 & & & 1 & 1 & & & & & & \\
\hline 9 & 1 & 1 & 2 & 1 & 3 & & & & & & \\
\hline 10 & & 1 & & & & & & & & & \\
\hline 11 & & & & & & 8 & 7 & 7 & 7 & 76 & 6 \\
\hline
\end{tabular}


Appendix K. Extra material from the Hospital B's case study from Chapter148

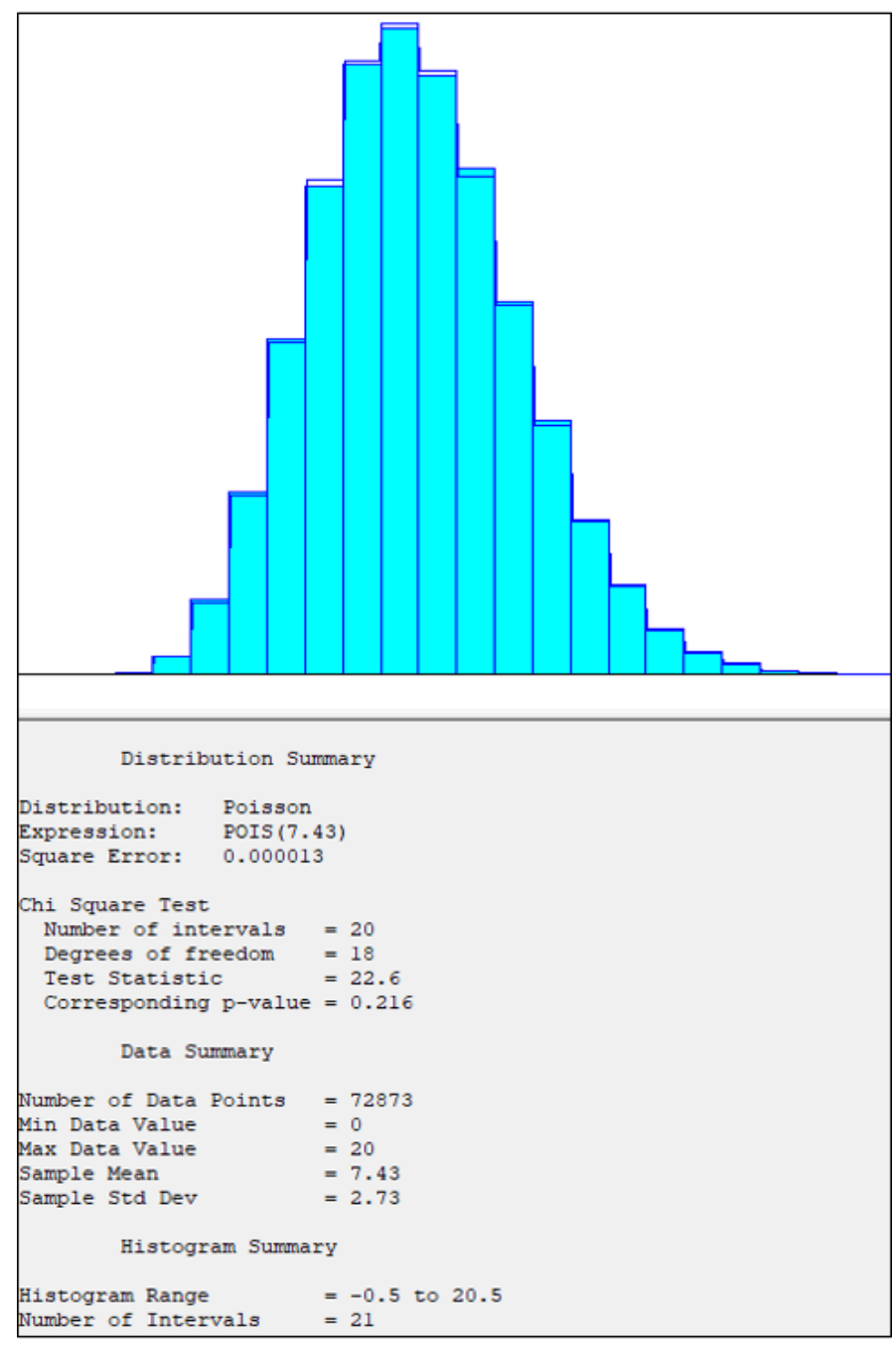

Figure K.1: Fit test of the patients arrival distribution in the Hospital A 
Appendix K. Extra material from the Hospital B's case study from Chapter149

Table K.2: Average interval between arrival per hour and day of week for first general assessment in ED of Hospital B

\section{Day}

\begin{tabular}{|c|c|c|c|c|c|c|c|}
\hline Time of day $(h)^{a}$ & $\mathbf{M}$ & $\mathbf{T}$ & $\mathbf{W}$ & $\mathbf{T}$ & $\mathbf{F}$ & $\mathbf{S}$ & $\mathbf{S}$ \\
\hline 0 & 23.5 & 19.2 & 18.7 & 18.7 & 20.7 & 18.4 & 17.1 \\
\hline 1 & 36.0 & 20.4 & 26.2 & 26.2 & 20.8 & 27.3 & 26.3 \\
\hline 2 & \multirow[b]{2}{*}{28.7} & & & & & & \\
\hline 6 & & 29.8 & 29.4 & 29.4 & 33.9 & 33.5 & 28.9 \\
\hline 3 & \multirow{3}{*}{41.3} & \multirow{3}{*}{35.9} & \multirow{3}{*}{41.0} & \multirow{3}{*}{41.0} & \multirow{3}{*}{46.0} & \multirow{3}{*}{38.0} & \multirow{3}{*}{32.4} \\
\hline 4 & & & & & & & \\
\hline 5 & & & & & & & \\
\hline 7 & \multirow[b]{2}{*}{7.5} & \multirow[b]{2}{*}{9.9} & \multirow[b]{2}{*}{10.8} & \multirow[b]{2}{*}{10.8} & \multirow[b]{2}{*}{11.4} & \multirow[b]{2}{*}{12.8} & \multirow[b]{2}{*}{13.6} \\
\hline 22 & & & & & & & \\
\hline 8 & 4.8 & 5.0 & 7.2 & 7.2 & 6.2 & 10.1 & 10.3 \\
\hline 9 & 3.7 & 4.1 & 5.1 & 5.1 & 5.2 & 6.2 & 5.8 \\
\hline 10 & \multirow{5}{*}{3.2} & \multirow{5}{*}{3.9} & \multirow{5}{*}{4.4} & \multirow{5}{*}{4.4} & \multirow{5}{*}{4.5} & \multirow{5}{*}{5.6} & \multirow{5}{*}{5.7} \\
\hline 11 & & & & & & & \\
\hline 13 & & & & & & & \\
\hline 14 & & & & & & & \\
\hline 15 & & & & & & & \\
\hline 12 & 3.7 & 4.1 & 5.1 & 5.1 & 5.2 & 6.2 & 5.8 \\
\hline 16 & \multirow[b]{2}{*}{4.1} & \multirow[b]{2}{*}{4.5} & \multirow[b]{2}{*}{4.7} & \multirow[b]{2}{*}{4.7} & \multirow[b]{2}{*}{5.4} & \multirow[b]{2}{*}{7.2} & \multirow[b]{2}{*}{6.5} \\
\hline 17 & & & & & & & \\
\hline 18 & \multirow[b]{2}{*}{4.5} & \multirow[b]{2}{*}{5.2} & \multirow[b]{2}{*}{5.5} & \multirow[b]{2}{*}{5.5} & & & \\
\hline 19 & & & & & 6.3 & 7.7 & 7.1 \\
\hline 20 & & & & & & & \\
\hline 21 & 6.1 & 6.3 & 6.4 & 6.4 & 7.9 & 8.6 & 8.9 \\
\hline 23 & 10.4 & 11.6 & 11.2 & 11.2 & 11.6 & 13.5 & 13.1 \\
\hline
\end{tabular}

$\mathrm{a}_{24}$ hour clock format 


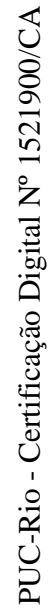


$\mathbf{L}$

\section{Extra material from the case study of Chapter 5}

Table L.1: Average interval between arrival per hour and day of week in ED of hospital studied

\section{Day}

\begin{tabular}{|c|c|c|c|c|c|c|c|}
\hline Time of day $(h)^{a}$ & M & $\mathbf{T}$ & $\mathbf{W}$ & $\mathbf{T}$ & $\mathbf{F}$ & $\mathbf{S}$ & $\mathbf{S}$ \\
\hline 0 & 21.2 & 17.9 & 16.8 & 16.8 & 18.6 & 16.6 & 17.1 \\
\hline 1 & 32.4 & 18.4 & 23.6 & 23.6 & 18.7 & 24.6 & 23.8 \\
\hline 2 & \multirow[b]{2}{*}{25.8} & \multirow[b]{2}{*}{26.8} & \multirow[b]{2}{*}{26.5} & \multirow[b]{2}{*}{26.5} & \multirow[b]{2}{*}{30.5} & \multirow[b]{2}{*}{30.1} & \multirow[b]{2}{*}{26.0} \\
\hline 6 & & & & & & & \\
\hline 3 & \multirow{3}{*}{37.2} & \multirow{3}{*}{32.3} & \multirow{3}{*}{36.9} & \multirow{3}{*}{36.9} & \multirow{3}{*}{41.4} & \multirow{3}{*}{34.2} & \multirow{3}{*}{29.2} \\
\hline 4 & & & & & & & \\
\hline 5 & & & & & & & \\
\hline 7 & \multirow[b]{2}{*}{6.8} & \multirow[b]{2}{*}{8.9} & \multirow[b]{2}{*}{9.7} & \multirow[b]{2}{*}{9.7} & \multirow[b]{2}{*}{10.2} & \multirow[b]{2}{*}{11.5} & \multirow[b]{2}{*}{12.2} \\
\hline 22 & & & & & & & \\
\hline 8 & 4.3 & 4.5 & 6.5 & 6.5 & 6.2 & 9.1 & 9.27 \\
\hline 9 & 3.3 & 3.7 & 4.6 & 4.6 & 4.7 & 5.6 & 5.2 \\
\hline 10 & \multirow{5}{*}{2.9} & \multirow{5}{*}{3.5} & \multirow{5}{*}{3.9} & \multirow{5}{*}{3.9} & \multirow{5}{*}{4.0} & \multirow{5}{*}{5.0} & \multirow{5}{*}{5.1} \\
\hline 11 & & & & & & & \\
\hline 13 & & & & & & & \\
\hline 14 & & & & & & & \\
\hline 15 & & & & & & & \\
\hline 12 & 3.3 & 3.7 & 4.6 & 5.1 & 4.7 & 5.6 & 5.2 \\
\hline 16 & \multirow[b]{2}{*}{3.7} & \multirow[b]{2}{*}{4.2} & \multirow[b]{2}{*}{4.2} & \multirow[b]{2}{*}{4.2} & \multirow[b]{2}{*}{4.9} & & \\
\hline 17 & & & & & & 6.5 & 5.9 \\
\hline 18 & & & & & & & \\
\hline 19 & 4.1 & 4.7 & 4.6 & 4.6 & 5.7 & 6.9 & 6.4 \\
\hline 20 & & & & & & & \\
\hline 21 & 5.5 & 5.7 & 5.8 & 5.8 & 7.1 & 7.7 & 8.0 \\
\hline 23 & 9.4 & 10.4 & 10.0 & 10.0 & 10.4 & 12.2 & 11.8 \\
\hline
\end{tabular}


M

Size of the model from the Hospital B's case study from Chapter 5

The size of the models (i.e., variables and constraints), average execution times, and their variances are shown in Table M.1.

Table M.1: Size of SAA's experiments from Chapter 5

\begin{tabular}{ccccc}
\hline Scenarios & Variables & Constraints & Time(s) & St.Dev(s) \\
\hline $\mathbf{2 0}$ & 57835 & 351553 & 676.36 & 117.39 \\
$\mathbf{4 0}$ & 98155 & 411893 & 1618.82 & 516.90 \\
$\mathbf{6 0}$ & 138475 & 472233 & 2550.01 & 475.38 \\
$\mathbf{8 0}$ & 178795 & 532573 & 3383.81 & 282.93 \\
$\mathbf{1 0 0}$ & 219115 & 592913 & 3602.60 & 0.34 \\
\hline
\end{tabular}


N

\section{Size of the model from Chapter 6}

The size of the models (i.e., variables and constraints) and the average execution times are shown in Table N.1.

\begin{tabular}{|c|c|c|c|}
\hline $\mathbf{N}^{\mathbf{a}}$ & Variables & Constraints & Time(s) \\
\hline 5 & 23,702 & 29,178 & 517.21 \\
\hline 10 & 46,437 & 57,473 & 795.62 \\
\hline 15 & 69,172 & 85,768 & $1,215.75$ \\
\hline
\end{tabular}

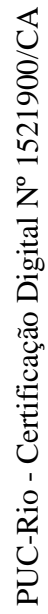

${ }^{\mathrm{a}} \mathrm{N}$ - Number of Scenarios 


\section{0 \\ Extra material from the Chapter 6}

Table O.1 summarize the results in terms of the average queue, worst case, and average idle capacity for clinic and surgery service for each value of $\beta$ factor. In this table, we present the average numbers followed by the standard deviation between parenthesis. We report the total average and the average related just to the last month of planning horizon, this is because in the CRS just in the last month (Month 5) of the planning horizon considered we can do free assignments (as explained in Section 6.3.1) and the model has freedom to assign the physicians according to the objective. Before that, the schedule used is the same, although in month 3, the decision regard assignment to the second OR also varies for each objective. 
Table O.1: Results summary about average queue, capacity and patients seen in clinic and surgery service

\begin{tabular}{|c|c|c|c|c|c|c|c|c|c|c|c|c|}
\hline & & & & & & & & & & & & \\
\hline & Indicators & 0 & 0.1 & 0.2 & 0.3 & 0.4 & 0.5 & 0.6 & 0.7 & 0.8 & 0.9 & 1 \\
\hline \multirow{5}{*}{ Clinic } & Average Queue (Dev.) & $682.49(392.03)$ & $52.83(31.72)$ & $33.85(30.53)$ & $26.71(25.64)$ & $10.05(8.29)$ & $5.10(4.99)$ & $4.23(4.62)$ & $4.01(4.37)$ & $3.19(4.12)$ & $2.81(3.82)$ & $2.36(3.76)$ \\
\hline & Worst Case & 1489.00 & 178.00 & 133.00 & 121 & 97.00 & 52.00 & 80.00 & 71.00 & 165.00 & 48.00 & 43.00 \\
\hline & Average Queue in the Last Month (Dev.) & $1188.29(94.12)$ & $22.24(15.13)$ & $7.35(6.02)$ & $4.48(4.21)$ & $1.96(1.83)$ & $1.58(1.93)$ & $0.88(0.89)$ & $1.22(1.78)$ & $0.31(0.41)$ & $.24(0.41)$ & $0.64(2.19)$ \\
\hline & Worst Queue in the Last Month & 1489.00 & 178.00 & 62.00 & 52.00 & 42.00 & 26.00 & 79.00 & 69.00 & 52.00 & 25.00 & 10.00 \\
\hline & Average Idle Capacity (Dev.) & $17.38(7.66)$ & $6.76(5.29)$ & $5.79(4.88)$ & $5.59(4.71)$ & $5.76(4.28)$ & $5.79(4.42)$ & $5.64(4.38)$ & $5.63(4.56)$ & $5.98(4.86)$ & $5.86(4.87)$ & $9.12(9.23)$ \\
\hline \multirow{5}{*}{ Surgery } & Average Queue (Dev.) & $13.24(35.44)$ & $20.89(35.09)$ & $23.83(35.92)$ & $26.67(37.83)$ & $36.94(45.89)$ & $41.71(48.02)$ & $41.74(47.94)$ & $43.00(47.49)$ & $44.31(47.27)$ & $46.91(46.56)$ & $548.49(228.65)$ \\
\hline & Worst Case & 159.00 & 159.00 & 159.00 & 159.00 & 160.00 & 163.00 & 164.00 & 163.00 & 48.00 & 167.00 & 1051.00 \\
\hline & Average Queue in the Last Month (Dev.) & $0.03(0.02)$ & $0.76(1.04)$ & $1.33(1.36)$ & $2.33(2.02)$ & $4.07(3.84)$ & $5.88(4.45)$ & $5.38(4.22)$ & $6.35(5.09)$ & $7.39(5.37)$ & $8.77(5.79)$ & $845.71(60.36)$ \\
\hline & Worst Queue in the Last Month & 53.00 & 8.00 & 11.00 & 12.00 & 31.00 & 47.00 & 47.00 & 43.00 & 16.00 & 47.00 & 1051.00 \\
\hline & Average Idle Capacity (Dev.) & $7.94(3.67)$ & $1.96(1.83)$ & $1.76(1.66)$ & $1.84(1.69)$ & $1.76(1.82)$ & $1.76(1.76)$ & $1.67(1.68)$ & $1.58(1.62)$ & $1.54(1.59)$ & $1.43(1.54)$ & $7.83(3.45)$ \\
\hline \multirow{4}{*}{ Total } & Average Queue (Dev.) & $695.73(372.77)$ & $73.73(51.82)$ & $57.68(56.50)$ & $53.38(55.82)$ & $46.99(50.23)$ & $46.81(48.53)$ & $45.97(40.87)$ & $47.02(48.18)$ & $47.51(47$. & $49.72(47$. & $550.85(227$ \\
\hline & Worst Case & 1497.00 & 212.00 & 205.00 & 211.00 & 211.00 & 189.00 & 190.00 & 189.00 & 187.00 & 188.00 & 1054.00 \\
\hline & Average Queue in & $1188.26(94.13)$ & $22.99(15.46)$ & $8.67(6.54)$ & $6.81(5.51)$ & $6.04(5.21)$ & $7.45(5.10)$ & $6.26(0.89)$ & $7.57(5.79)$ & $7.71(5.76)$ & $9.01(5.88)$ & $845.77(60.47)$ \\
\hline & Worst Queue in the Last Month & 1497.00 & 182.00 & 67.00 & 63.00 & 65.00 & 62.00 & 123.00 & 104.00 & 63.00 & 66.00 & 1054.00 \\
\hline
\end{tabular}

